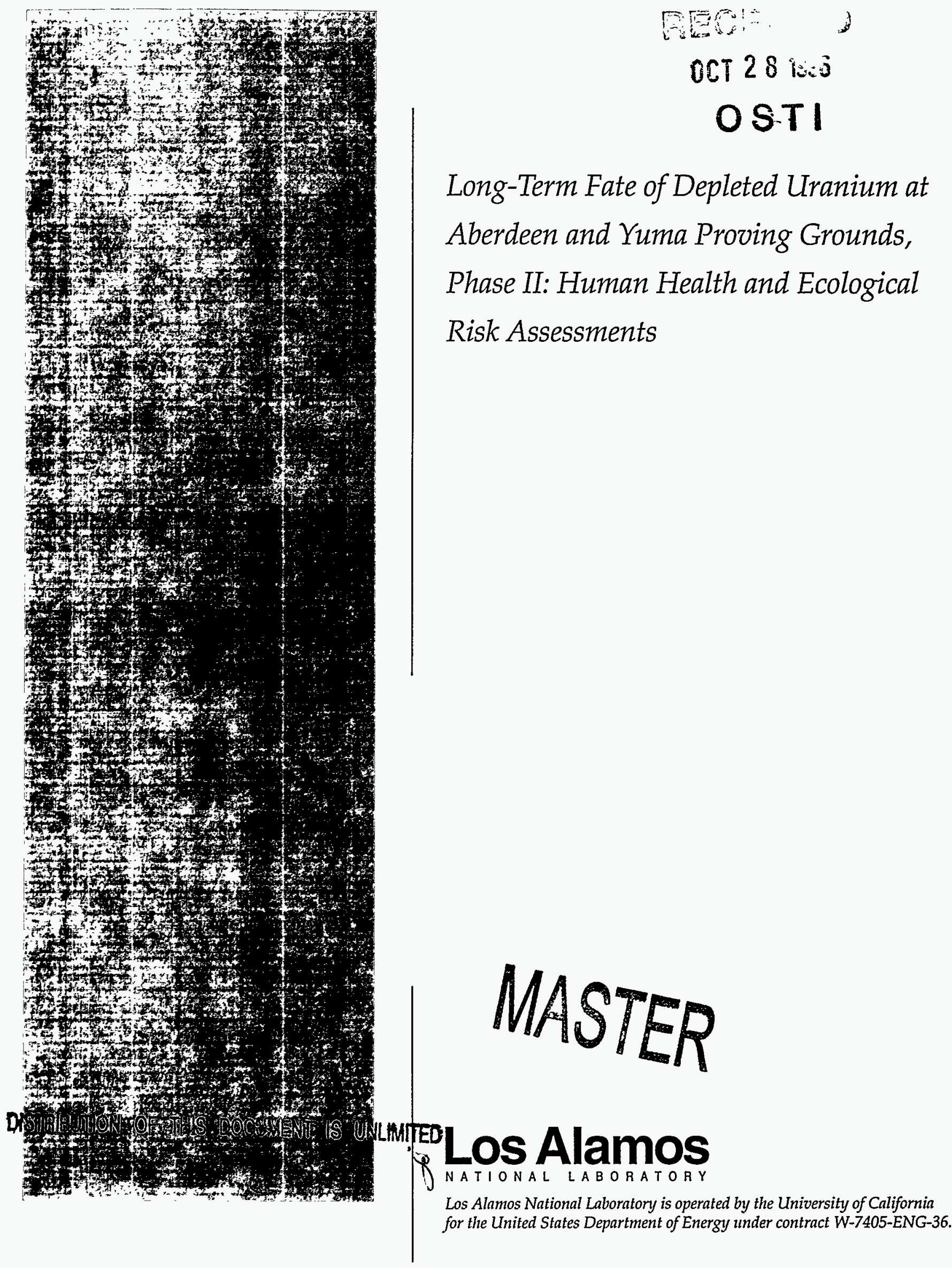


An Affirmative Action/Equal Opportunity Employer

This report was prepared as an account of work sponsored by an agency of the United States Government. Neither The Regents of the University of Californin, the United States Government nor any agency thereof, nor any of their employees, makes any warranty, express or implied, or assumes any legal liability or responsibility for the accuracy, completeness, or usefulness of any information, apparatus, product, or process disclosed, or represents that its use would not infringe privately owned rights. Reference herein to any specific commercinl product, process, or service by trade name, trademark, manufacturer, or otherwise, does not necessarily constitute or imply its endorsement, recommendation, or favoring by The Regents of the University of California, the United States Government, or any agency thereof. The views and opinions of authors expressed herein do not necessarily state or reflect those of The Regents of the University of California, the United States Government, or any agency thereof. The Los Alamos National Laboratory strongly supports academic freedom and a researcher's right to publish; therefore, the Laboratory as an institution does not endorse the viewpoint of a publication or guarantee its technical correctness. 


\section{DISCLAIMER}

Portions of this document may be illegible in electronic image products. Images are produced from the best available original document. 


\section{Long-Term Fate of Depleted Uranium at}

\section{Aberdeen and Yuma Proving Grounds,}

\section{Phase II: Human Health and Ecological}

\section{Risk Assessments}

Michael H. Ebinger*

Patricia L. Kennedy**

Orrin B. Myers**t+

William Clements**

Heidi T. Bestgen**

Richard J. Beckmant

\section{DISCLAIMER}

This report was prepared as an account of work sponsored by an agency of the United States Government. Neither the United States Government nor any agency thereof, nor any of their employees, makes any warranty, express or implied, or assumes any legal liability or responsibility for the accuracy, completeness, or usefulness of any information, apparatus, product, or process disclosed, or represents that its use would not infringe privately owned rights. Reference herein to any specific commercial product, process, or service by trade name, trademark, manufacturer, or otherwise does not necessarily constitute or imply its endorsement, recommendation, or favoring by the United States Government or any agency thereof. The views and opinions of authors expressed herein do not necessarily state or reflect those of the United States Government or any agency thereof.

*Environmental Science Group, Los Alamos National Laboratory

"Department of Fishery and Wildlife Biology, Colorado State University, Ft. Collins, CO

'Statistics Group, Los Alamos National Laboratory

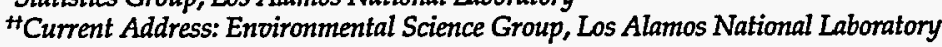

\section{Los Alamos}

NATIONAL LABORATORY

Los Alamos, New Mexico 87545 


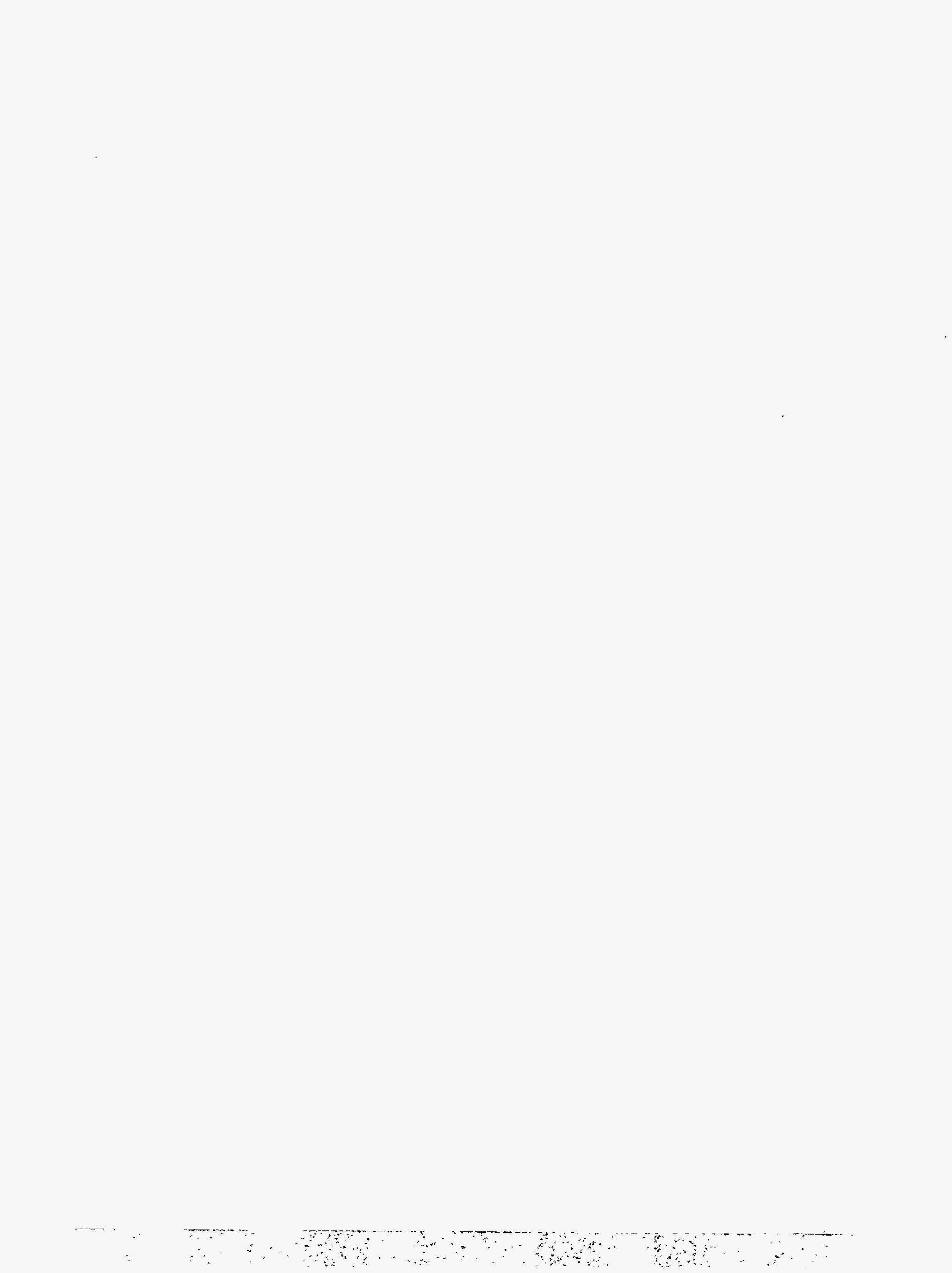




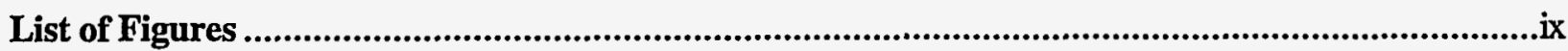

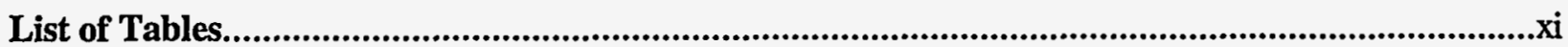

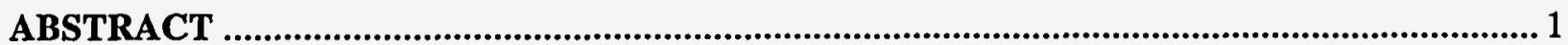

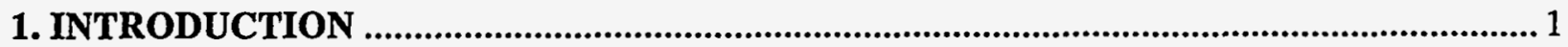

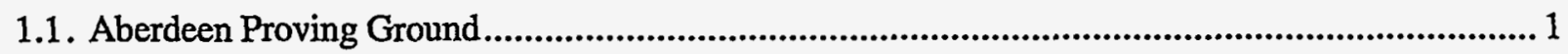

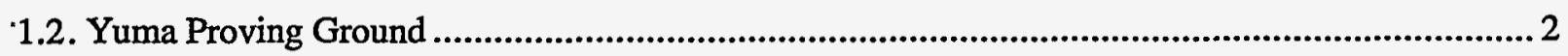

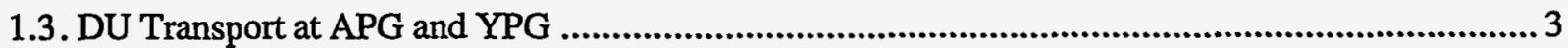

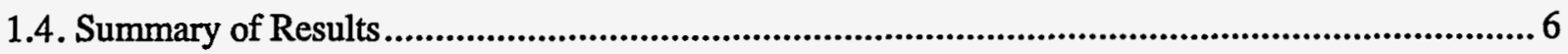

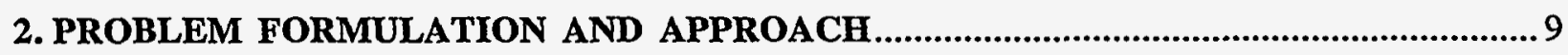

3. ECOLOGICAL STUDIES AND MODELING AT APG ............................................13

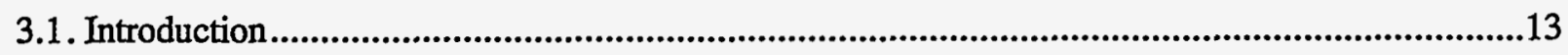

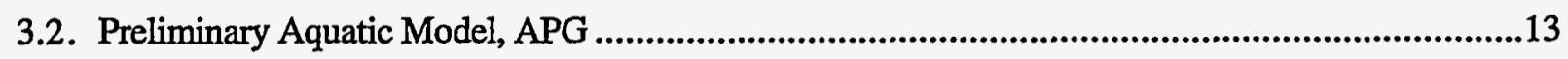

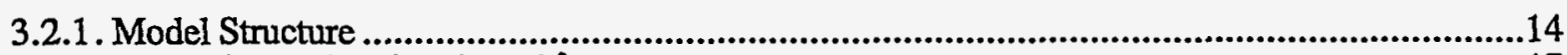

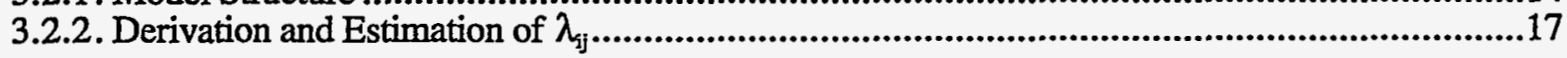

3.3. Model Uncertainty and Sensitivity Analysis ............................................................................18

3.4. Results of Modeling ............................................................................................................22

3.4.1. Model Uncertainty and Sensitivity Analyses-Aquatic Model...........................................22

3.4.1.1. Uniform Input Distributions .......................................................................................22

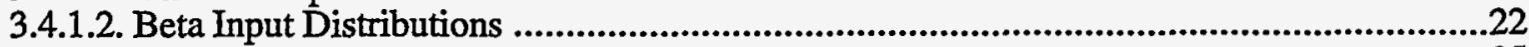

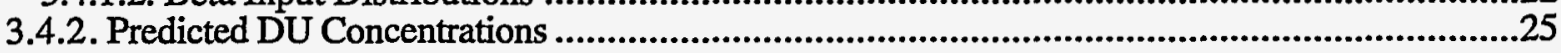

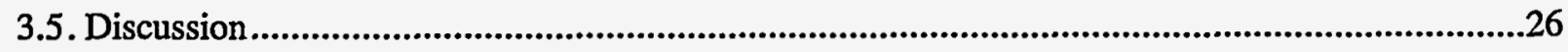

3.5.1. Model Uncertainty and Sensitivity Analyses.............................................................26

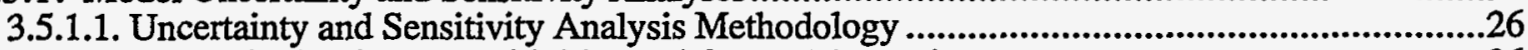

3.5.1.2. Input Distributions, Sensitivities and Output Uncertainty................................................26

3.5.2. Predicted DU Concentrations .................................................................................................27

3.5.3. A Strategy for Reducing Uncertainty of Predicted Exposures...................................................28

3.6. Steady-State Model, APG Terrestrial Ecosystem..........................................................................30

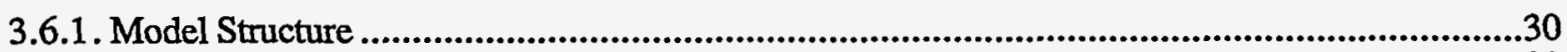

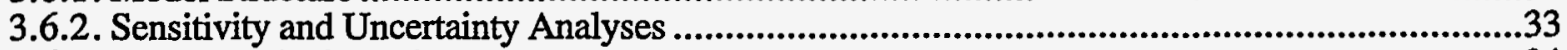

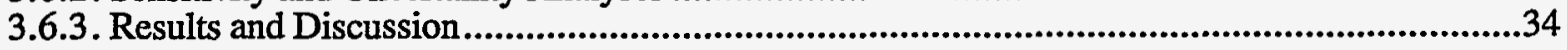




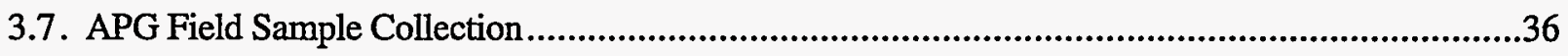

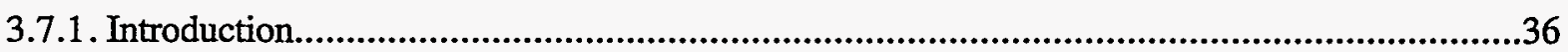

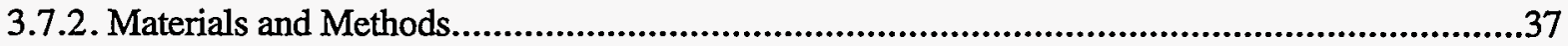

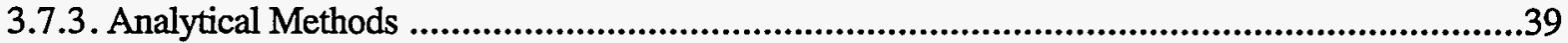

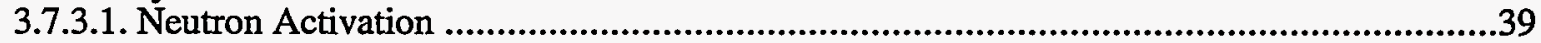

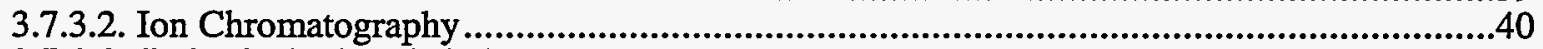

3.7.3.3. Inductively Coupled Plasma-Mass Spectroscopy......................................................41

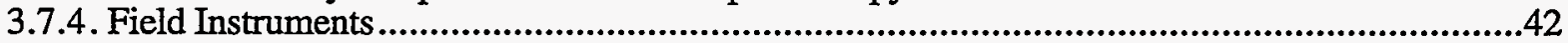

3.7.5. Water Chemistry and Stream Flow Measurements ........................................................42

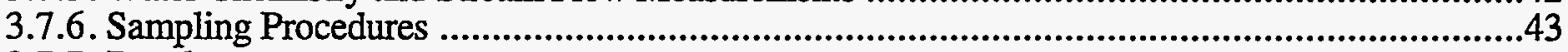

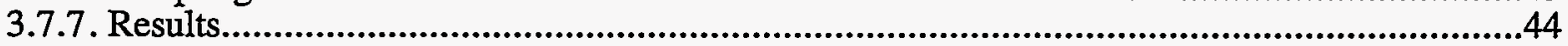

3.7.7.1. Groundwater and Surface Water .............................................................................44

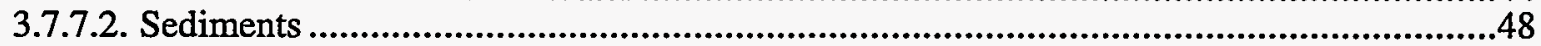

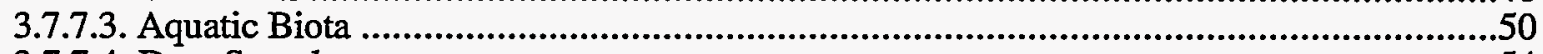

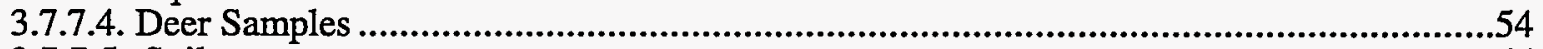

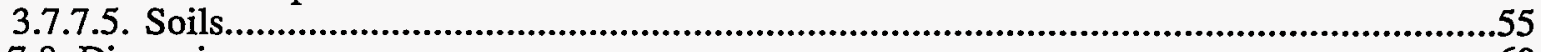

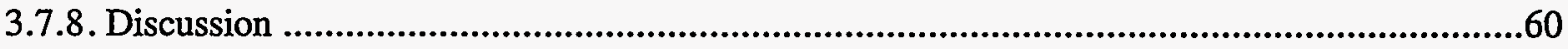

4. ECOLOGICAL STUDIES AND MODELING AT YPG ...............................................63

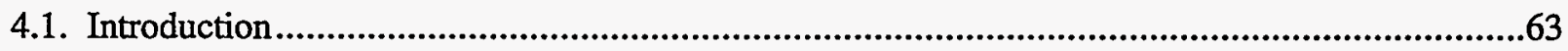

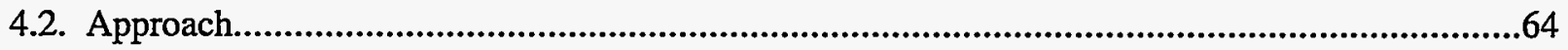

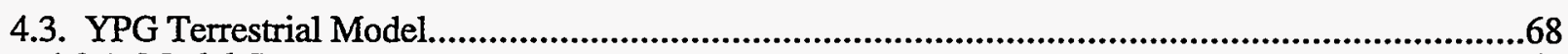

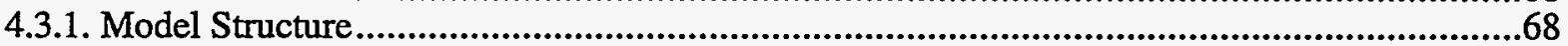

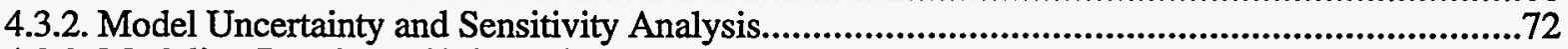

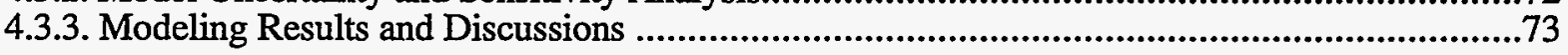

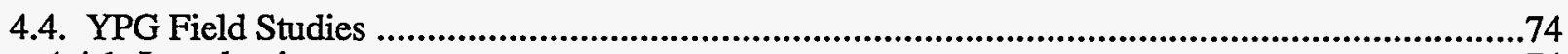

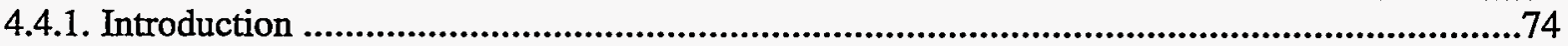

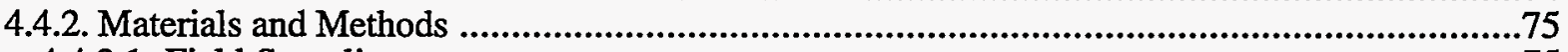

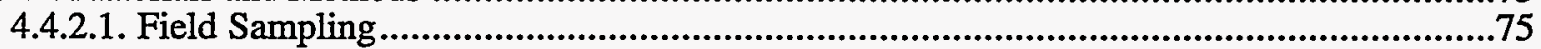

4.4.2.2. Kangaroo Rat Histopathology .....................................................................................77

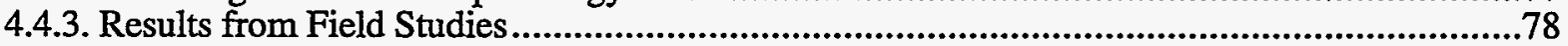

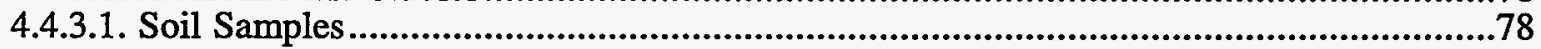

4.4.3.2. Vegetation and Litter Samples ...................................................................................78

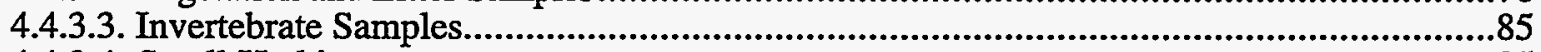

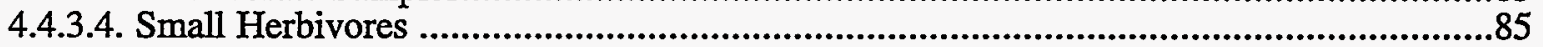

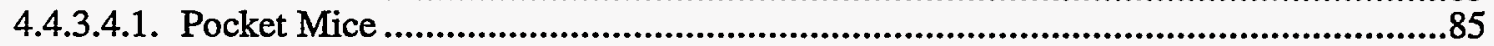

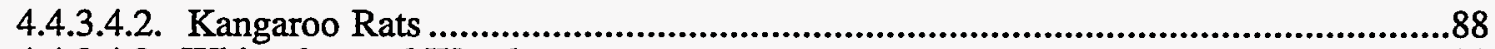

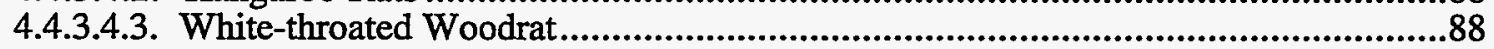

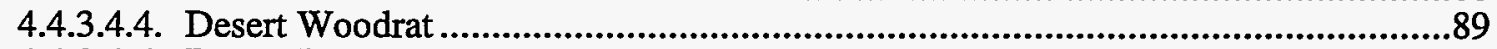

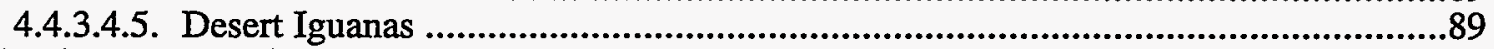

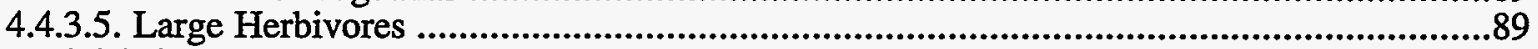

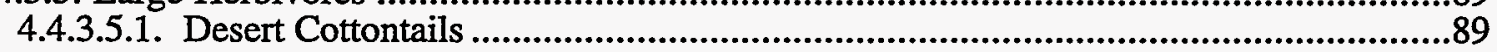

4.4.3.5.2. Black-tailed Jack Rabbit...........................................................................................90

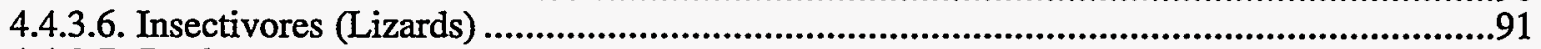

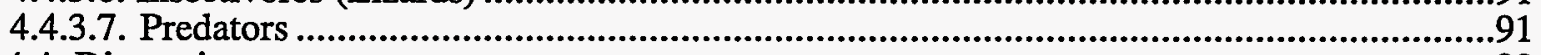

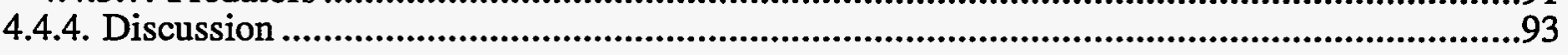


5.1. Dose Calculations for Human Health Effects ..........................................................................95

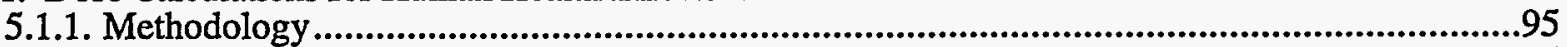

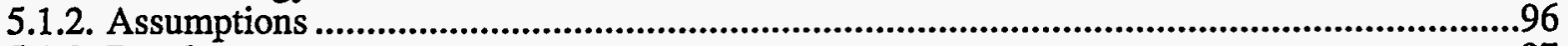

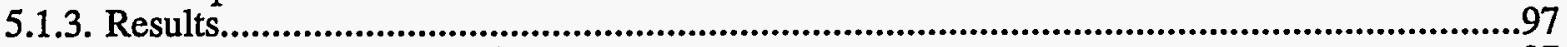

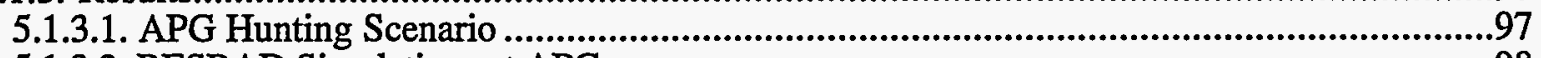

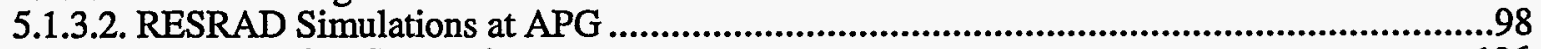

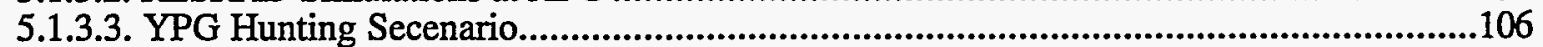

5.1.3.4. RESRAD Simulations at YPG ............................................................................107

5.1.3.5. Toxicological Doses to Humans...................................................................................109

5.2. Dose Calculations to Ecosystem Components ..........................................................................109

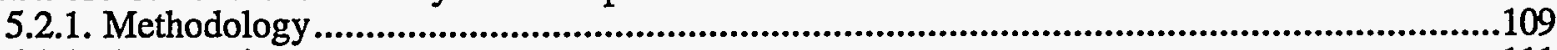

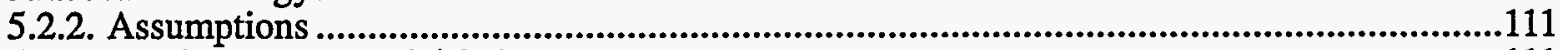

5.2.3. Results: Ecosystem Risk Assessment .................................................................111

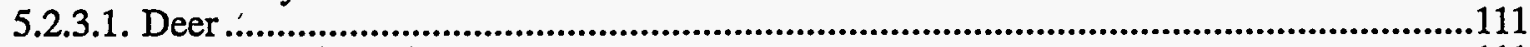

5.2.3.2. Sunfish and Perch .......................................................................................................111

5.2.3.3. Kangaroo Rats and Pocket Mice .............................................................................112

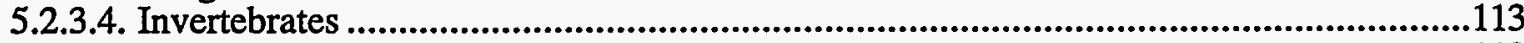

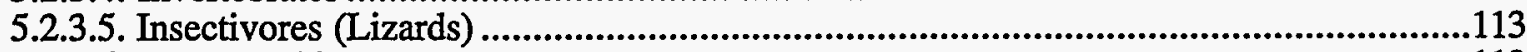

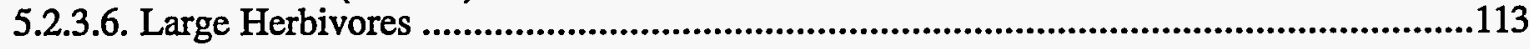

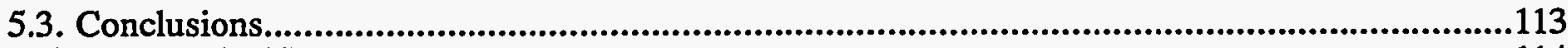

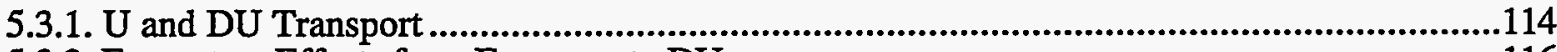

5.3.2. Ecosystem Effects from Exposure to DU .....................................................................116

5.3.3. Human Health Effects .................................................................................................116

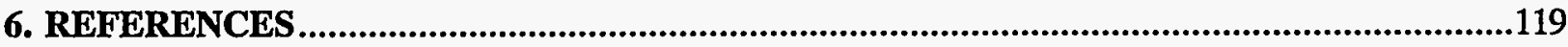

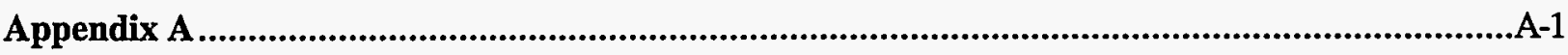

Appendix B

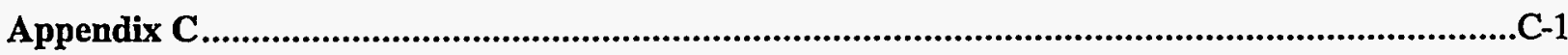

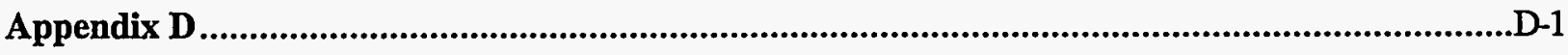

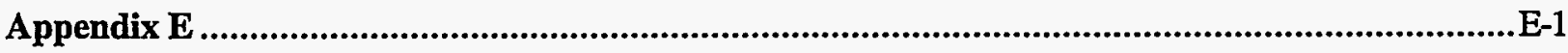

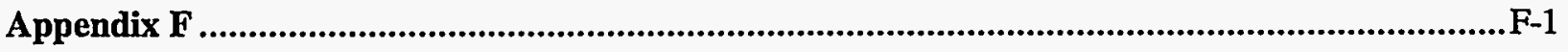





\section{LIST OF FIGURES}

Figure 1-1. Schematic of the method of approach used for this study ...............................................5

Figure 3-1. Conceptual model of uranium transport through the aquatic food web at Aberdeen Proving Ground. Based on carbon flux model of Baird and Ulanowicz (1989). Representative organisms for compartments (or feeding categories) are listed in Table 3-1. Arrows in the diagram indicate potential DU flow or transport to different compartments. Arrows to W, SD, and $\mathrm{E}$ represent losses from compartments to water, sediment detritus, and export compartments, respectively.

Figure 3-2a. Relative uncertainty of exposure model compartments measured by the square root of the sum of squared input variable sensitivity values. Input values were generated from uniform and beta distributions as indicated. OM is organic matter, BSF is benthic suspension feeders, BDF is benthic deposit feeders, and SFF is suspension feeding fish.

Figure 3-2b. Relative uncertainty of compartments when input values were aggregated by their roles in the exposure model. Abiotic parameters describe transport between abiotic compartments, structural parameters describe the standing crop biomass structure of the food web, functional parameters describe the rates of material exchange between its compartments, BCF were bioconcentration factors used to calculate compartment concentrations at equilibrium, and physiological parameters included compartment ventilation rates, elimination rates, and DU assimilation rates. Uncertainty due to water concentration was not included.

Figure 3-3. DU transport pathways identified from sensitivity analysis. Solid line indicates major importance to model output, dashed line indicates intermediate to minor importance.

Figure 3-4. Schematic of the Steady State Model. Model shows DU transfer to deer from drinking water, soil ingestion, and plant ingestion pathways and transfer to humans from deer tissue consumption. ...30

Figure 3-5. Sampling locations at APG in July 1992 and October 1992.............................................38

Figure 3-6. Probability distribution of U concentration in sediments from APG. Approximately $82 \%$ of all sediment concentrations are $0.6 \mathrm{pCi} / \mathrm{g}$ or less. ...............................................................................5

Figure 3-7. Probability distribution of $U$ in sunfish samples from APG. .............................................53

Figure 3-8. Probability distribution for $U$ in perch from APG. ............................................................53

Figure 3-9. Soil transect results at $6500 \mathrm{~m}$ downrange on the Main Front Firing Range line of fire. Negative values refer to distances east (left) of the firing line, positive values refer to locations west (right) of the firing line. ...........................................................................................58

Figure 3-10. Distribution of the soil concentrations of $U$ or DU from APG environmental monitoring samples from 1980 through 1990 . See Table C-6 for concentrations $>50 \mathrm{pCi} / \mathrm{g}$.

Figure 4-1. A. Spatial distribution of DU at YPG, GP 20. B. Spatial distribution of DU at YPG, GP 17A. In both diagrams, the firing line is at $0 \mathrm{~m}$, positive values on the $\mathrm{N}-\mathrm{S}$ Distance axis are north of the firing line, and negative values are south of the firing line. Downrange distance is the distance from the gun position along the firing line.

Figure 4-2. A. Spatial distribution of ${ }^{234} U{ }^{238} U$ activity ratios from GP 20, YPG. B. Spatial distribution of ${ }^{234} U{ }^{238} \mathrm{U}$ activity ratios from GP $17 \mathrm{~A}$, YPG. In both diagrams, the firing line is at $0 \mathrm{~m}$ along the $\mathrm{N}-\mathrm{S}$ Distance axis, positive values are north of the firing line, and negative values are south of the firing line. Downrange distance is the distance from the gun position along the firing line.

Figure 4-3. Annual precipitation at YPG, 1959 and 1966 through 1991

Figure 4-4. Average monthly precipitation at YPG, 1959 and 1966 through 1991 ................................67

Figure 4-5. Conceptual model of DU transport through the environment at YPG

Figure 4-6. Layout of pitfall traps used to sample invertebrates, reptiles, and amphibians. Pitfall diameters were approximately $14 \mathrm{~cm}$ amd $9 \mathrm{~cm}$ 
Figure 4-7. Schematic diagram of biotic and abiotic samples collected from five locations along GP 17A and GP 20 firing lines, August 1992.

Figure 4-8. Distribution of $U$ concentrations in YPG soil samples ..................................................83

Figure 4-9. Distribution of $U$ concentrations in unwashed vegetation $(A)$ and litter $(B)$ collected at YPG.

Figure 4-10. Distribution of U concentrations in invertebrates collected at YPG. Concentrations based on fresh tissue weight

Figure 4-11. Distribution of $U$ concentrations in pocket mice carcasses (A) and kidneys (B). Concentrations based on fresh tissue weight in both plots. BDL (B) indicates that concentrations of ${ }^{238} \mathrm{U}$ and ${ }^{235} \mathrm{U}$ were below detection limits.

Figure 4-12. Distribution of $U$ concentrations in lizard samples. Concentrations based on fresh tissue weight in both plots

Figure 5-1. Surface water (A) and groundwater (B) concentrations of $U$ at APG predicted with RESRAD. Initial $U$ concentration is $0.85 \mathrm{pCi} / \mathrm{L}$ in (A) and $850 \mathrm{pCi} / \mathrm{L}$ in (B).

Figure 5-2. Doses from RESRAD with $\mathrm{DU}$ solubility of $0.85 \mathrm{pCi} / \mathrm{L}$ (low solubility). Surface water and groundwater are included in the water-dependent pathways line 105

Figure 5-3. Doses from RESRAD with DU solubility of $850 \mathrm{pCi} / \mathrm{L}$ (high solubility). Surface water and groundwater are included in the water-dependent pathways line.

Figure 5-4. Total dose and dose by different pathways from RESRAD simulations with soil $\mathrm{U}$ concentration of $16 \mathrm{pCi} / \mathrm{g}$. Total dose is the summation of doses from all pathways .

Figure 5-5. Total dose and dose by different pathways from RESRAD simulations of YPG with a soil concentration of $83 \mathrm{pCi} / \mathrm{g}$. Total dose is the summation of doses from all pathways 


\section{LIST OF TABLES}

Table 3-1. Ecological compartments of a carbon flow model in the mesohaline region of Chesapeake Bay (Baird and Ulanowicz, 1989) and corresponding compartments in the DU exposure model (Myers et al. 1993).

Table 3-2. Relative sensitivities of calculated DU concentrations $\left(C_{i}\right.$ in $\left.\mathrm{mg} / \mathrm{kg}\right)$ and inventories $\left(\mathrm{Q}_{\mathrm{i}}\right.$ in $\left.\mathrm{mg} / \mathrm{m}^{2}\right)$ to exposure model input variables when input values were drawn from uniform and beta probability distributions. Appendix $A$ is a list of input values for all parameters.

Table 3-3. DU concentrations calculated from aquatic exposure model. Inputs drawn from beta distributions. All values are $\mathrm{mg} / \mathrm{kg}$.

Table 3-4. Various statistics from the uncertainty analysis of the steady-state model for predicted values of $\mathrm{C}_{\mathrm{i}}$.

Table 3-5. Contributions of the six parameters that most effect $C_{i}$, the concentration of DU in deer tissue. The results of 10,000 iterations were used to generate the distribution of the predicted values.............35

Table 3-6. Water quality data for surface water at APG. Data collected July, 1992.................................45

Table 3-7. Water quality data collected October 1992 from sites at APG.............................................46

Table 3-8. Data from wells collected 4/4/93.

Table 3-9. Flow rate data for Mosquito Creek near the Trench Warfare Range (TW) catch box and at the mouth. Volume flow rates are averages of three repeat measurements, standard deviation is in parentheses.

Table 3-10. Uranium concentrations in surface water and groundwater from APG. Numbers in parentheses indicate number of samples used to compute the average and standard deviations.

Table 3-11. Sediment samples collected at APG and analyzed by neutron activation or ICP-MS. Since ${ }^{238} \mathrm{U}$ is activated to ${ }^{239} \mathrm{U}$ in NAA, ${ }^{239} \mathrm{U}$ activities are reported instead of ${ }^{238} \mathrm{U}$. A ${ }^{235} \mathrm{U} /{ }^{239} \mathrm{U}$ activity ratio less than 0.8 for NAA data indicates the presence of $D U$, whereas values greater than 1.0 indicate natural uranium. In the ICP-MS data, $a^{235} \mathrm{U} /{ }^{238} \mathrm{U}$ mass ratio $<0.0045$ indicates the presence of DU whereas a ratio greater than 0.0065 indicates natural U. Site names in italics and bolded indicate that at least one sample contained DU. See Figure 3-6 for map locations of the sites.

Table 3-12. Concentration of $U$ by species from APG samples. Average and standard deviation given in $\mathrm{pCi} / \mathrm{g}$ of wet tissue. Species in bold italics indicate at least one measurement with $U$ from $\mathrm{DU}$. Remaining species either had insufficient data to calculate the ${ }^{235} \mathrm{U} /{ }^{238} \mathrm{U}$ ratio or the ratio indicated natural U. Additional data by species in Appendix C.

Table 3-13. U Concentrations of various compartments of the aquatic model. Average and standard deviation given in $\mathrm{pCi} / \mathrm{g}$ of wet tissue. Italics indicate $\mathrm{DU}$ identified in at least one sample.

Table 3-14. Data from deer sampling program. Off-site deer were collected on the Eastern Shore and in Cecil County, Maryland. Impact area deer were collected near the firing line of the Main Front Firing Range.

Table 3-15. Soil concentrations of U from APG locations. Samples were collected between July 1992 and September 1993.

Table 4-1. Nominal and maximum DU concentrations predicted for ecosystem compartments by the YPG transport model

Table 4-2. Numbers and types of samples collected and analyzed from YPG GP 20 and GP 17A.

Each compartment of the YPG terrestrial model is represented so that redistribution of

DU from soils through the food web could be estimated. 
Table 4-3. Descriptive statistics for U concentrations in YPG samples. All values in $\mathrm{pCi} / \mathrm{g}$ except as noted for kidney samples. Sample names in bold italics indicate that DU was detected in at least one measurement. DL indicates the detection limit. Appendix E lists all data condensed in this table

Table 5-1. Radiological doses to humans from consumption of deer tissue calculated from the steady-state model. Consumption rate assumes that humans replace all dietary meat with deer tissue and consume approximately $95 \mathrm{~kg}$ of meat per year. Mean values calculated from the model are shown with the $95 \%$ confidence intervals

Table 5-2. Radiological doses to humans from consumption of deer tissue calculated from deer field data. Consumption rate assumes that humans replace all dietary meat with deer tissue and consume approximately $95 \mathrm{~kg}$ of meat per year. Mean values and $95 \%$ confidence intervals are shown

Table 5-3. Radiological doses to humans from consumption of sunfish and perch tissue. Consumption rate assumes that sunfish and perch are the sole sources of fish in the diet and that all the fish came from impact-area streams.

Table 5-4. Radiological doses to humans from consumption of shellfish (blue crab). Consumption rates range from 1 to $15 \mathrm{~kg}$ of crab tissue per year in the simulations.

Table 5-5. Radiological doses to humans from all sources (deer tissue, fish, shellfish, drinking water, and soil ingestion).

Table 5-6. Expected annual detriments due to the DU exposures shown in Table 5-5. Detriments are considered fatal cancers, nonfatal cancers, and the occurrence of severe heredity effects (ICRP 1990).

Table 5-7. Expected detriments from the high-solubility and low-solubility simulations using RESRAD. Values are the number of detriments expected per $10^{6}$ or $10^{5}$ people, as indicated. For example, $7.0 \times 10^{-6}$ indicates there are 7 detriments expected per $10^{6}$ people.

Table 5-8. Doses to humans from consuming rabbit tissue from YPG. Values are within the upper 99\% confidence intervals. Doses were calculated using ingested-DU retention rates of $1 \%$ and $5 \%$

Table 5-9. Dose rates calculated from concentrations of $U$ and DU in various compartments of the APG and YPG ecosystems 


\title{
LONG-TERM FATE OF DEPLETED URANIUM AT ABERDEEN AND YUMA PROVING GROUNDS, PHASE II: HUMAN HEALTH AND ECOLOGICAL RISK ASSESSMENTS
}

\author{
by
}

Michael H. Ebinger, Patricia L. Kennedy, Orrin B. Myers, William Clements, Heidi T. Bestgen, and Richard J. Beckman

\begin{abstract}
The purpose of this study was to evaluate the immediate and long-term consequences of depleted uranium (DU) in the environment at Aberdeen Proving Ground (APG) and Yuma Proving Ground (YPG) for the Test and Evaluation Command (TECOM) of the U.S. Army. Specifically, we examined the potential for adverse radiological and toxicological effects to humans and ecosystems caused by exposure to DU at both installations. We developed contaminant transport models of aquatic and terrestrial ecosystems at APG and terrestrial ecosystems at YPG to assess potential adverse effects from DU exposure. Sensitivity and uncertainty analyses of the initial models showed the portions of the models that most influenced predicted DU concentrations, and the results of the sensitivity analyses were fundamental tools in designing field sampling campaigns at both installations. Results of uranium (U) isotope analyses of field samples provided data to evaluate the source of $U$ in the environment and the toxicological and radiological doses to different ecosystem components and to humans. Probabilistic doses were estimated from the field data, and DU was identified in several components of the food chain at APG and YPG. Dose estimates from APG data indicated that U or DU uptake was insufficient to cause adverse toxicological or radiological effects. Dose estimates from YPG data indicated that $U$ or DU uptake is insufficient to cause radiological effects in ecosystem components or in humans, but toxicological effects in small mammals (e.g., kangaroo rats and pocket mice) may occur from $U$ or DU ingestion. The results of this study were used to modify environmental radiation monitoring plans at APG and YPG to ensure collection of adequate data for ongoing ecological and human health risk assessments.
\end{abstract}

\section{INTRODUCTION}

\subsection{Aberdeen Proving Ground}

Aberdeen Proving Ground (APG) is a U.S. Department of Defense-designated Major Range Test Facility Base on the western shore of Chesapeake Bay. APG has served as a major military testing and training facility since 1917 , with $80 \%-85 \%$ of the area being composed of ballistic test ranges, impact areas, vehicle test tracks, and other test facilities. As a result of depleted uranium (DU) penetrator munitions testing programs begun in the 1950s, DU has been deposited over approx. 1500 acres at APG. Most penetrator impacts occurred within about $500 \mathrm{~m}$ of the firing axis after the DU munitions passed through soft targets used to check accuracy and performance. Penetrators strike the ground, trees, and wetlands after hitting soft targets and eventually come to rest in the impact area. About $20 \%$ of all DU fired has been physically recovered and removed from the firing range, and the balance remains at the impact area. Recovery efforts were discontinued for safety reasons in 1992 and because catch boxes were constructed to minimize additional deposition and dispersal of penetrator fragments in the environment. 
The catch boxes stop over $90 \%$ of the rounds fired, significantly reducing the amount of DU added to the environment. The site is a restricted area and very likely will remain a dedicated testing facility for the foreseeable future. This study estimates the potential adverse effects to the environment of the present inventory of DU in the environment and the potential adverse effects of continued testing.

APG and adjacent areas provide habitat for a variety of terrestrial and aquatic life, some of which has commercial or recreational value and some that is protected by federal statutes. These habitats have been affected by anthropogenic disturbances not associated with APG (Mackiernan 1990). Aquatic and terrestrial life at APG show no negative effects due to the DU penetrator testing (U.S. Army Combat Systems Test Activity 1990a), although the uncertainty in the Army's assessment has not been determined. Given the long-lasting nature of potential DU hazards (Cothern et al. 1983; Kocher 1989; Leggett 1989), the likelihood that DU will remain in the environment, and uncertainty about the ecological risks of DU, continuing environmental monitoring and assessment are warranted if negative ecological effects are to be avoided. Risk managers also need estimates of potential adverse effects and the amount of error in the estimates in order to understand and manage ecological risks of APG operations.

\subsection{Yuma Proving Ground}

Testing of modern military equipment began in Yuma about January 1943 when the U.S. Army Corps of Engineers opened the Yuma Test Branch near the Colorado River. The primary mission was to test new bridge designs, boats, vehicles, and well-drilling equipment during World War II. Flow from Imperial Dam was regulated to provide an ideal test environment for bridges and boats. The area was also used to train soldiers for service in World War II's North African desert campaigns under the leadership of General George Patton. After a one-year break in service, the test facility was reactivated in 1952 as Yuma Test Station. The principal mission was to conduct desert environmental tests of military equipment. In 1962 the Yuma Test Station was reassigned to the U.S. Army Materiel Command and placed under the immediate control of the Test and Evaluation Command (TECOM). The test station was renamed the Yuma Proving Ground in 1963, and in 1973 it was renamed the U.S. Army Yuma Proving Ground (YPG). YPG was designated a Department of Defense Major Range and Test Facility in 1974 and was licensed by the Nuclear Regulatory Commission (NRC) in 1982 for the testing of DU penetrators.

YPG is a lower Sonoran desert ecosystem that has been affected by the testing mission. Terrestrial wildlife, however, show no adverse effects from the DU penetrator testing or from other testing in the vicinity of the DU test ranges. Continuous monitoring of DU within the YPG reservation confirms that little exposure of humans to DU is expected and that the concentrations of DU in soils and vegetation are restricted to testing areas. Again, risk managers need estimates of potential adverse effects and the amount of error in the estimates in order to understand and manage ecological risks of YPG operations. 


\subsection{DU Transport at APG and YPG}

In 1989 and 1990, TECOM was interested in the potential transport of DU through soils, sediments, and water at APG and YPG. Studies conducted at that time found that (1) DU migrates vertically at APG to at least $20 \mathrm{~cm}$ in soils because of precipitation adequate to cause chemical transport at APG; (2) DU migrates by way of soil erosion at YPG; (3) no DU was detected in shallow groundwater or surface water at APG, and no DU was detected in the aquifer that underlies YPG; and (4) sediments at APG showed some DU, whereas at YPG DU was measured in the wash sediments but deposition from erosion processes was not uniform (Ebinger et al. 1990). Shortly after these results were published, TECOM contracted for the current, larger study to examine the possible effects of residual DU on the ecosystems of APG and YPG, as well as for potential adverse human health effects caused by ingestion of DU via harvested animals. As penetrator fragments weather or corrode at APG and YPG, the DU is released to the environment and is potentially available for uptake by different ecosystem components. Based on the amount of DU remaining on the test ranges, the present study addresses the following questions:

- Is DU migrating through soils of the impact areas at APG and YPG?

- Is DU migrating into Chesapeake Bay at APG or off-site at YPG?

- Does DU adversely affect ecosystems at APG or YPG?

- Does residual DU at APG or YPG adversely affect human health?

TECOM wants to ensure that human health and the ecosystems at APG and YPG do not suffer from adverse effects from the DU munitions testing programs. Approximately $70,000 \mathrm{~kg}$ of DU fragments were in the impact areas of each site at the beginning of the present study. Since then, several measures have been implemented to reduce or eliminate further deposition and dispersal of DU fragments in the soils and waters of APG and YPG. Among those measures are removal of DU fragments during collection operations and construction and use of catch boxes at APG and soon at YPG. The catch boxes will minimize the amount of DU deposited in the impact areas during future testing operations, but the DU deposited before the implementation of the catch boxes at APG and deposited to date at YPG is still a potential risk to the ecosystems and humans in the areas.

DU may adversely affect the health of plants and animals living in the impact areas or using the areas surrounding the impact areas to procure food and water. Areas of interest at APG include the terrestrial environment and the aquatic environment. Soils at APG are clearly affected by high water tables as shown by extensive gray mottles within about $10 \mathrm{~cm}$ of the soil surface. A series of groundwater monitoring wells accesses water from about 1-4 $\mathrm{m}$ below the soil surface along the firing line of the Main Front Firing Range. Both freshwater and brackish water ecosystems exist and are potentially affected by DU munitions testing. In addition, permitted hunting and fishing at APG for fish, shellfish, deer, and other game animals provide potential pathways for DU ingestion by humans.

The lower Sonoran desert environment at YPG is a complex terrestrial ecosystem that could be affected by the DU munitions testing programs. No hunting program exists at YPG, and a direct pathway to humans is not likely. Therefore, most of the emphasis in this study was placed on the potential risk to the ecosystem from fragments of DU deposited in soils and eroded into washes. The groundwater system 
at YPG is at least $200 \mathrm{~m}$ below the surface of the impact area and is not a transport pathway of major concern. There is no permanent surface water at YPG, and only occasionally do the washes carry runoff from rainstorms.

With the above objectives in mind, we designed this study to estimate the potential adverse effects on the ecosystems and humans in the impact areas. The overall approach is shown in Figure 1-1. The initial phase was to evaluate the information that was on hand before beginning extensive sampling. Environmental sampling at APG and YPG provided initial estimates of soil, sediment, surface water, and groundwater concentrations, as did previous studies (Ebinger et al. 1990). The ecosystems were also evaluated so that the animal and plant communities of interest were known. Once the ecosystems were qualitatively inventoried, preliminary pathway models were developed to show potential routes of DU transport through the aquatic and terrestrial food chains. The scientific literature was searched for all information on the effects of $U$ or DU on plants and animals. The information of interest included transfer factors that would allow estimation of $U$ or DU migration from one ecosystem compartment to another and actual uptake rates of $U$ or DU by plants or animals. When the necessary information was not found, approximations from other sites were made. These assumptions will be discussed in more detail later in this report.

Food-chain models allowing DU transport were developed, and the initial concentration data and information about ecosystem composition and dynamics were used to model the potential transport of DU through the ecosystems. Estimates of DU concentrations in individual components of the ecosystems were calculated to complete the preliminary assessment.

Uncertainty in the estimates was compared with the estimates with a single question in mind: are the uncertainties small enough that the risk assessment could terminate at this point? Uncertainties for most estimates ranged from five to seven orders of magnitude; therefore, the uncertainties were far too large to conclude the risk assessment without additional sampling.

The next phase was to begin refinement of the assessment models and to design a field sampling strategy that would provide data to reduce the uncertainties in the assessment models. Sampling of soils, sediments, vegetation, and aquatic and terrestrial animals was conducted at APG and YPG. Over 2500 samples of various media were analyzed for DU and used for the risk assessment. The data collected allowed us to construct probability distributions of DU concentrations in the medium of interest and to use these distributions in the assessment models. The data also allowed estimation of doses to animals and vegetation independently of the assessment models. For example, data from deer collected at APG and on the Eastern Shore of Maryland provided a data set to compare with model results and to use for dose estimates. 


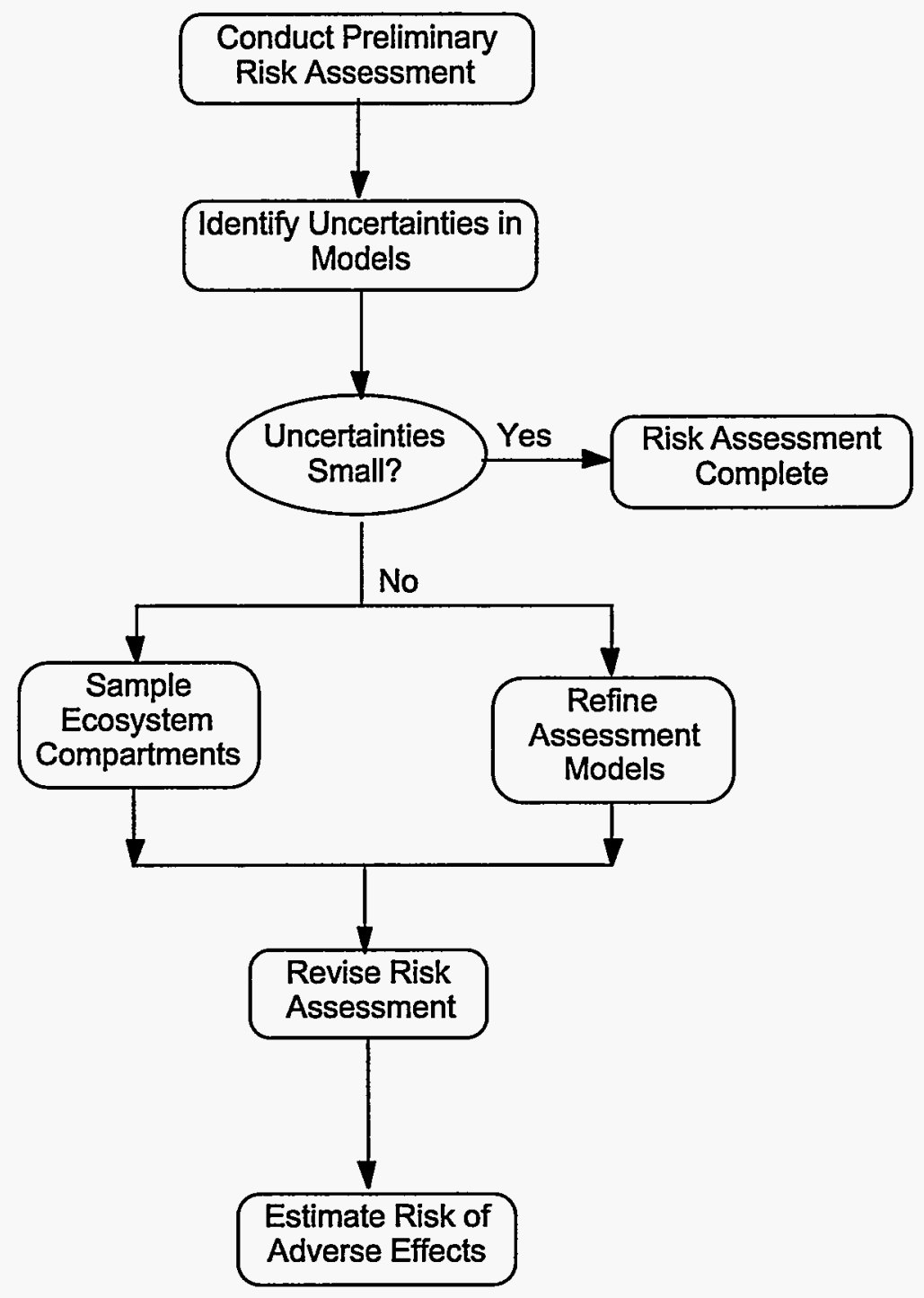

Figure 1-1. Schematic of the method of approach used for this study. 
Model refinements included removing or modifying pathways in the models that did not contribute significantly to the estimates and adding pathways that did significantly contribute. These refinements were aided by sensitivity analyses performed on the models. For example, the deposition of airborne DU on plant surfaces was underestimated in the initial steady state model and was modified in later versions because of the importance of this pathway to the amount of DU ingested by deer.

The field data and refined models led to a revised risk assessment using all available data. The uncertainties were greatly reduced over the preliminary estimates and were more meaningful for further risk management decisions. The revised assessment provided the information necessary to estimate ingestion of DU by animals and humans and thus to convert ingestion by humans and animals into estimates of potential risks of fatal and nonfatal cancers and nephrotoxicity. The risk estimates and the corresponding doses are discussed in detail later in this report.

\subsection{Summary of Results}

The data presented in Chapters 3 and 4 and the dose estimates and risk evaluations in Chapter 5 show the following:

- measurable $U$ is present in most samples;

- DU is present in most compartments of the YPG ecosystem but is not as prevalent in the APG ecosystem;

- U could not always be attributed unambiguously to DU or natural $U$ because the ${ }^{235} U$ concentrations were often below the detection limit;

- radiological doses to humans and ecosystem components were insignificant; and

- toxicological doses to humans and those ecosystem components that could be tested showed that kidney burdens of $U$ were well below assumed thresholds for renal damage in most ecosystem compartments, except for kangaroo rats from YPG, for which histopathological investigations detected possible damage to kidney tissue.

Data in the following chapters suggest that few of the compartments in the ecosystem are adversely affected by DU uptake, although $U$ was detected in most compartments and often could not be assigned unambiguously to DU or natural U. The only data supporting possible adverse effects were collected from the small herbivore compartment of the YPG model. The main pathways by which DU may be transported from impact areas are the detritus and surface water pathways at APG and erosion at YPG. The presence of $U$ and DU in most ecosystem components of the affected areas at YPG, the concentrations of $U$ in aquatic endpoints in or near affected areas at APG, and the measurable $U$ in deer tissues in affected areas at APG and off-site emphasize the importance of monitoring the environment and assessing the potential of adverse effects to humans and ecosystems from DU fragments in the environment.

At APG rainfall provides sufficient moisture to transport corrosion products from DU fragments through soil profiles to depths of at least $20 \mathrm{~cm}$ and probably deeper. Data from groundwater wells in the impact area show $U$ and DU below detection limits, and no DU has been detected in groundwater in nearly 25 years of DU testing at APG. This trend could change with time because DU transport is slow 
and may require many years to reach the sampling volume of the monitoring wells. Subsurface transport of DU at APG is hindered for several reasons. First, the groundwater flow velocity is low (i.e., "inches per day" [U.S. Army Combat Systems Test Activity 1990b]) due to the low gradient in the aquifer. Without the gradient necessary to move groundwater at a higher velocity, the DU transport mechanism is also slow. Second, high water tables at APG are common, as seen in the mottled gray soil profiles throughout the impact area. Measurements of dissolved oxygen and oxidation-reduction potentials indicate the prevalence of reducing conditions, and there is ample visual evidence from soil mottling that shows that microbial activity in the soil is sufficient to reduce uranium to the $\mathrm{U}^{4+}$ state (Lovely et al. 1991). Uranium in the 4+ state is about three orders of magnitude less soluble and significantly less mobile than $\mathrm{UO}_{2}^{2+}$, the common form of soluble $U$ in the $6+$ state. Thus, there is high probability that DU mobility is limited by the precipitation of $\mathrm{U}^{4+}$ solids within the wet soils and in the wetlands surrounding APG. There is also a high probability that precipitation of $U^{4+}$ is enhanced by microbial activity. Third, most of the material in the penetrator fragments is $U$ metal. Penetrator fragments corrode and are released to the soil-water system (Erikson et al. 1993), and the amount of DU entering the soil system depends on the corrosion rate of the fragments. The inventory of DU in the soils, therefore, is released over a long time period and is not available to plants and animals all at once. One of the main uncertainties affecting the amount of DU transported through the environment is the rate at which the fragments corrode and release DU. We incorporated only rudimentary rate information in the assessments reported here because developing rate data was well beyond the scope of this study.

Erosion at YPG is the primary mechanism of DU transport. DU in washes adjacent to impact areas has been observed (e.g., Ebinger et al. 1990), but attempts to systematically sample DU in washes have met with little success. Ward and Stevens (1994) show that desert pavements provide the necessary conditions for collecting the limited rainfall at YPG and channeling it to cause erosion. However, DU movement by way of erosion is limited because most desert pavements have insufficient gradients to cause movement of DU-containing soil. While some of the inventory will erode into the washes, the majority of the material will be retained on desert pavements. Reducing conditions in the soils do not exist at YPG, and DU transport is not limited in that way. However, the low annual rainfall limits the transport of DU into the soil profiles. While the corrosion rates of DU fragments seem to be greater at YPG than APG, the potential for transporting the corrosion products depends on the amount and timing of rain. High-intensity storms are required to create surface flow conditions sufficient to move DU fragments on desert pavement. Adequate storms occur infrequently in the summer and rarely at other times during the year.

Wind deposition is a secondary mode of transport for DU at YPG. Wind dispersal of dust that contains DU was observed in the field during penetrator testing. However, wind blowing across the impact areas is considered a minor DU transport mechanism because it does not disturb DU-containing dust as frequently as munitions testing does. DU-containing dust was found during field surveys and from laboratory samples as coatings on vegetation surfaces, and these data were incorporated into the assessments reported in this study. 
Erosion at YPG deposits DU from runoff into washes that dissect the impact areas, and the washes are potential conduits for moving DU off-site. Sampling for DU in washes revealed concentrations of $U$ and DU greater than detection limits only in washes with known (i.e., visible) DU. The total volume of sediment (without DU) carried by washes dilutes or masks the DU to below detection limits, effectively decreasing the concentration of DU transported off-site. The tortuosity of the washes provides numerous depositional locations within the YPG reservation for DU eroding from impact areas. The availability of numerous depositional areas within washes suggests that finding areas of locally high DU concentrations (i.e., "hot spots") is of low probability and suggests further that high concentrations of DU do not move off-site. DU eroded into washes adjacent to impact areas must be transported through a series of smaller washes before reaching Castle Dome Wash, and sufficient flow in the washes must exist before transport occurs. Limited rainfall of sufficient intensity to cause channel flow limits the distance of DU transport. Thus, the probability of DU moving beyond YPG boundaries is low and depends on the frequency and intensity of rainfall events in the watershed drained by Castle Dome Wash.

The remainder of this report is presented in the following sections: problem formation is discussed in Chapter 2; APG model development and results are discussed in Chapter 3; YPG models and results are discussed in Chapter 4; and risk estimates and conclusions are presented in Chapter 5. The appendices following the text supply the data used in various parts of the report. 


\section{PROBLEM FORMULATION AND APPROACH}

The general thrust of this project was to provide an assessment of the fate of DU in the environment at APG and YPG. Several attempts to state the overall goal in an operational framework involved input from many people working with the DU test mission at APG and YPG. Ultimately, as much information as possible was sought to address the following questions:

- Is DU migrating through soils of the impact areas at APG and YPG?

- Is DU migrating into Chesapeake Bay at APG or off-site at YPG?

- Does DU adversely affect ecosystems at APG or YPG?

- Does residual DU at APG or YPG adversely affect human health?

These objectives were condensed from several interviews with APG and YPG personnel about what each thought the project ought to be concerned with. The above objectives were the consensus of those interviewed and were confirmed at a meeting of APG, YPG, and Los Alamos personnel early in the project. The interviews ensured that all people involved in the project contributed to the way the work was conducted, and they focused the efforts of all involved on a common set of objectives. The knowledge gained from the interviews was an important factor during sampling both at APG and YPG.

Our approach to providing the information to address the objectives was iterative (Figure 1-1). We adopted this approach for several reasons. First, there was no readily available set of modeling or assessment tools that would allow adequate estimation of human health effects and ecological risk at either APG or YPG. Instead, we modified existing models to fit our needs or developed new models as required. The results are a set of models that are highly site-specific to APG and YPG.

Second, we were not certain initially about the most appropriate way to sample the APG and YPG ecosystems to evaluate the effects of DU. The models allowed us to examine uncertainty in estimates of exposures and to examine the exposure pathways that most influenced the estimates. Examining the uncertainty in the input data and the uncertainty apparent from different calculations showed the kinds of processes and samples that needed to be included in the assessment in order to minimize the uncertainty in the dose estimates. Evaluating the sensitivity also showed several processes that were less important than we thought, as well as ecological processes that were more important and were not included adequately in the initial models. The iterative approach provided several opportunities to include different samples and/or modify the models to adequately describe the pertinent ecological processes.

Third, the iterative approach collected information on DU concentrations in different media at different stages of the project. Our approach provided subsets of the data that were useful for preliminary dose and risk estimates throughout the course of the study. For example, we wanted to know early in the sampling campaign if there were acute risks to the ecosystem and to humans using the impact area during hunting season. Had acute risks been observed in the field or suggested from the data, APG and YPG would have taken appropriate actions to minimize additional DU exposure to animals whose home ranges include the impact area and to humans. However, no acute effects were observed or suggested from the data. 
Selecting appropriate endpoints for this risk assessment was essential before sampling or exposure modeling began. Human health endpoints are relatively easy to select since radiation protection is designed to protect individual humans. Individuals, either radiation workers or members of the public not routinely near anthropogenic radiation sources, were endpoints in this study. Ecological endpoints, however, were more difficult to select. Historically, threatened or endangered species were the only individuals considered in ecological risk assessments. At present, however, effects that could alter population structure or function are finding application as endpoints in ecological risk assessments. There is some guidance on the population effects of radiation suggesting that an exposure of $100 \mathrm{mrad} /$ day or less will not adversely affect the reproductive capability of plants and animals (IAEA 1992), but systematic study of radiation effects on populations in different ecosystems has been minimal. In this report we examine the radiation doses to individual mammals, fish, shellfish, and selected vegetation in order to evaluate the potential for adverse effects on the ecosystem as a whole. While this approach does not give definitive information on all possible effects to the populations that comprise the ecosystems at APG and YPG, our data show the expected exposures and subsequent doses to animals and vegetation that use the impact areas or areas adjacent to the impact areas. Data evaluated against the IAEAsuggested exposure limit of $100 \mathrm{mrad} /$ day and against human dose limits provide the framework for evaluating the potential effects of radiation on animals at APG and YPG.

Ingested DU is unique because it is a radiation hazard and a chemical hazard. Literature on the chemical effects of $U$ (and DU, by default) on the kidney shows that, especially for DU, the radiation hazard may be less of a consideration than the chemical effects (Cothern et al. 1983; Kocher 1989; Leggett 1989). Therefore, we were also interested in estimating kidney burdens on animals as well as concentrations in other tissues. Information on population effects of DU is again minimal, and our estimates were be made using the individual animal approach. Human kidney burdens were estimated for APG since there is a plausible pathway for DU to humans via annual deer hunting at APG.

We developed several models of DU transfer through the food webs at APG and YPG. The aquatic model for APG was based on a carbon-flow model of Baird and Ulanowicz (1989) and the terrestrial models for APG and YPG were developed from ecosystem considerations and observations made in the field at both locations. Development and use of each model will be discussed in Chapters 3 and 4 .

The models were developed and tested initially with available data and values from the literature for most parameters. The models at this stage were not specific to APG or YPG but were general models describing a number of different ecosystems. Because of the more general approach, there was significant uncertainty in estimates of doses to animals, vegetation, and humans. Initial calculations varied by as much as seven orders of magnitude and could not be used effectively without site-specific data and modifications to the way we modeled ecological processes. The first iteration showed how poorly the models performed with general data but indicated that site-specific data could significantly reduce the uncertainty. Thus, the significant result of the initial iterations was a focused environmental sampling plan that provided key data for meaningful risk estimates. 
The finite resources available for sample analysis underscored the importance of carefully selecting the samples to include in this study. We needed to ensure that enough samples were collected to represent the species or ecosystem compartment of interest and still leave enough resources for each compartment that required sampling. The approach adopted was to weight each compartment by its importance in the model used for assessment. This approach used the information gained from the sensitivity analyses to help select the most important compartments. The greatest weight was placed on the compartments that needed the best representation to reduce uncertainty in the estimates. For example, data on DU concentrations in suspension feeding fish were more heavily weighted than the DU concentrations in deep sediments simply because the deep sediments were a part of the model that did not impact other compartments as heavily as the suspension feeding fish compartment. The sampling design then called for more-intensive sampling from the suspension feeding fish than the deep sediment compartment. The compartments of the aquatic and terrestrial ecosystems at APG and of the terrestrial ecosystem at YPG were weighted in this way before sampling began.

We also wanted to evaluate predictions of the models against data collected from field specimens. For the APG aquatic ecosystem, laboratory tests were designed to show the effects of DU uptake on phytoplankton and zooplankton in controlled conditions. Difficulties with sample preparation in the plankton and food-chain tests, however, resulted in either no data collection or data of unusable quality. A controlled feeding experiment with kangaroo rats was designed to test potential effects of DU in the YPG terrestrial ecosystem. Histopathological examinations revealed lesions on some kangaroo rat kidney tissue, but adverse effects could not be correlated with dose because of difficulties with the sample preparation and analyses.

Field samples of deer muscle, bone, kidney, and liver tissue were collected and provided data on deer that lived within the impact area and on deer that were not affected by DU fragments in the environment. The deer data allowed comparison of models with field data and provided a data set for dose calculations to humans independent of a pathway model. Two sample populations of deer were collected, one set (25 deer) from the impact area at APG and a second set ( 5 deer) from an unaffected area on the Eastern Shore of Maryland. In order to model uptake of food and water by deer, several parameters, such as the amount of food consumed daily by deer, were estimated from literature values or were extrapolated from data on uptake by other animals. Excretion of $U$ or DU by deer was not explicitly modeled but was included as a factor that adjusted the total amount of $U$ or DU in deer at equilibrium. The results of the deer sampling and modeling of DU uptake are discussed in detail in Chapter 3.

Field data were used to estimate doses to animals from ingesting DU and to humans from ingesting animals that contained DU in the consumed tissues. The assessment models were used, and dose calculations were based on assumed amounts of consumption. The doses to animals were then compared to the IAEA dose limit so the potential for DU-induced adverse effects could be estimated. In addition, we assumed that the same dose limits for humans could be applied to animals and evaluated the doses using human radiation protection limits. Human exposure was estimated from assumed consumption of animal tissue, and the doses were converted to risk estimates. 
Finally, the results of the ecological and human health risk assessments reported in this document were used to modify the existing environmental monitoring plans for YPG and APG and are discussed in the appropriate documents (Ebinger and Hansen 1994a, 1994b, 1995). Some of the results of the risk assessment were the weights applied to the different ecosystem compartments during the sampling campaign. These weights were incorporated into the routine sampling required as a condition of the license to use DU at APG and YPG. The modified environmental sampling plans will also allow the monitoring data to be used as risk assessment data in the future. Thus, routinely collected environmental data can be used for ongoing risk assessments based on the methodology reported here. 


\section{ECOLOGICAL STUDIES AND MODELING AT APG}

\subsection{Introduction}

One purpose of this study was to evaluate the magnitude and sources of ecological risk at APG. The portion of the study presented in this chapter concerns the potential effects of DU on the aquatic ecosystem at APG and, to a limited extent, on the terrestrial ecosystem.

DU is deposited as described previously during the testing of munitions: penetrators pass through target areas, impact the earth, and ricochet downrange. Penetrators ultimately come to rest either at the initial impact site or at several locations downrange. At APG, soils and potentially surface ponds are contaminated when penetrator fragments are deposited. Soil and DU particles remain in the impact crater and are ejected for varying distances, depending on particle size and impact energy. Initial impacts cluster behind targets so that contamination zones from individual impacts may overlap. DU fragments weather readily in the humid APG environment and release DU metal as the mineral schoepite $\left(\mathrm{UO}_{2}(\mathrm{OH})_{2} \bullet \mathrm{H}_{2} \mathrm{O}\right)$ or as soluble $\mathrm{UO}_{2}^{2+}$ species and complexes. Yellow fragments of weathered penetrators are visible when undergrowth is cleared away from the soil surface. Several hundred acres of the Main Front Firing Range contain residual DU from munitions testing. Soil concentrations range from less than ten parts per million in areas not affected by DU deposition to several hundred parts per million in impact craters, and DU fragments are often visible in some locations.

The widespread DU deposition is a potential source of exposure for the plants and animals of the area. Dissolution of the penetrator fragments or of the weathering products could result in transport of DU through the groundwater and surface water systems and makes DU available for uptake by plants and animals. DU is also potentially available for uptake by terrestrial plants and animals either through assimilation through plant roots or through ingestion of DU-containing soil. Further transport of DU is possible as animals and plants exposed to DU are consumed by animals higher in the food chain.

There are several pathways for DU transport to humans at APG through hunting and fishing within the reservation boundaries. Thus, humans and ecosystem components could be adversely affected by the residue of munitions testing. This chapter presents our approach to assessing the potential adverse effects on humans and the ecosystem from DU munitions testing and the results of the study.

\subsection{Preliminary Aquatic Model, APG}

DU in the environment has potential for causing adverse ecological and human health effects due to its toxicological and radiological properties (Kocher 1989; Leggett 1989). Natural U is $99.2746 \%{ }^{238} \mathrm{U}$ (physical half-life $\left.=4.5 \times 10^{9} \mathrm{yr}\right), 0.7200 \%{ }^{235} \mathrm{U}\left(7.1 \times 10^{8} \mathrm{yr}\right)$, and $0.0054 \%{ }^{234} \mathrm{U}\left(2.5 \times 10^{5} \mathrm{yr}\right)$ (Walker et al. 1977) and has a specific activity of about $7.1 \times 10^{5} \mathrm{pCi} / \mathrm{g}(26 \mathrm{kBq} / \mathrm{g})$. DU, in comparison, is $99.7956 \%$ ${ }^{238} \mathrm{U}, 0.2002 \%{ }^{235} \mathrm{U}$, and $0.0007 \%{ }^{234} \mathrm{U}$ and has a specific activity of about $3.6 \times 10^{5} \mathrm{pCi} / \mathrm{g}(13 \mathrm{kBq} / \mathrm{g})$ due to the lower activity of ${ }^{234} \mathrm{U}$ and ${ }^{235} \mathrm{U}$ in DU. Although radiation from uranium can cause lethal cancers when ingested or inhaled (Kocher 1989; Wrenn et al 1987); Leggett (1989) argues that chemical toxicity should be the basis of standards to protect humans. The threshold for toxic effects to the mammalian kidney may occur at concentrations of 1-3 $\mu$ g of $U$ per gram of kidney ( $\mu$ g-U/g-kidney), but it could be 
an order of magnitude lower if toxicity is indicated by increased urinary excretion of proteins and amino acids (Leggett 1989; SuLu and Zhao 1990). Humans appear to be more tolerant of renal injury than other animals (Leggett 1989), although the toxicity threshold for other organisms is less well understood.

Models to calculate ecological exposure and risk are hypotheses about the distribution and effects of environmental disturbances. Uncertainty about processes and parameters in these models can cause risk estimates to span several orders of magnitude (Lipton and Gillett 1991). However, it is only recently that point estimates of risk have been replaced with more realistic exposure assessments in which projected risk estimates also include the uncertainty in the prediction (Lipton and Gillett 1991; Bartell et al. 1992). Evaluation of the risk of adverse effects to Chesapeake Bay aquatic life due to the deposition and persistence of DU in the environment at APG is reported in this chapter. Adverse effects for the purpose of this ecological risk assessment are a kidney burden of $1 \mu \mathrm{g}$-U/g-kidney (or greater) for toxicological effects or a radiation dose of $100 \mathrm{mrad} /$ day (or greater) for radiological effects. Adverse effects for human health are incidence of fatal and nonfatal cancers or a kidney burden of $1 \mu \mathrm{g}$-U/g-kidney or greater. Our goal is to present an approach whereby a reliable exposure model may be produced. We used a bioenergetics-based food-web model to provide the foundation of an ecological exposure assessment for $\mathrm{DU}$, and we present the following in this section:

- a food-web model as our hypothesis of how DU is transported through the aquatic and terrestrial food webs at APG;

- descriptions of the uncertainty and sensitivity analyses we performed to identify model parameters and processes most influential in causing uncertainty in hypothesized DU concentrations in aquatic and terrestrial organisms at APG; and

- demonstrations of how these analyses can provide guidance for planning field and laboratory experiments that will reduce uncertainty in predicted DU concentrations in aquatic and terrestrial organisms.

Our work illustrates the value of using uncertainty and sensitivity analyses in an iterative approach to exposure/risk modeling and assessment (Norton et al. 1992).

\subsubsection{Model Structure}

We developed a food-web model (Myers et al. 1993) to describe our hypothesis about DU transport in the aquatic ecosystem adjacent to APG by adapting a carbon-flux model for the mesohaline (6-18 $\mathrm{g}$ of dissolved salt per kilogram of water) region of Chesapeake Bay (Baird and Ulanowicz 1989). APG is situated $20 \mathrm{~km}$ north of Baltimore, Maryland, near the northern boundary of the mesohaline region of Chesapeake Bay. Although APG is not mesohaline, the model was our working hypothesis about biotic DU transport because a more site-specific transport model does not exist. An assumption made within the working hypothesis is that $U$ and/or DU is a minor constituent in the food chain; that is, $U$ or DU is a contaminant in the aquatic system. We used the carbon-flux model to show uptake and elimination of carbon as the food source within the aquatic ecosystem. As carbon, or food, is consumed, $U$ or DU is also consumed because of its presence in the food source. Thus, we modeled the flow of $U$ or DU as part of the dynamics of carbon flow in the food chain. 
In developing the carbon-flux model, Baird and Ulanowicz (1989) calculated mean seasonal biomass for 36 compartments and the exchanges of carbon among them. Compartments are categories of animals or plants that have similar feeding habits (animals) or growth environments (plants). In their model, gross primary production of autotrophic organisms (phytoplankton and benthic algae) was assumed to equal combined net primary production and algal respiration. Net primary production was partitioned among consumers according to herbivores' diets and energy requirements. Intake rates of heterotrophs were balanced relative to their summed rates of secondary production, respiration, and egestion, where egestion was material ingested and released back to the environment as feces without assimilation. Catches of commercially harvested fish populations were used to estimate standing crop biomass and export. The carbon-flux model is deterministic and aggregates several species into compartments that correspond to dominant feeding strategies (see Figure 13 of Baird and Ulanowicz 1989). We represent our adaptation of this model (Myers et al. 1993) schematically in Figure 3-1, where compartments (boxes) correspond to species aggregates and flows between compartments (arrows) are pathways for DU transport. We calculated the amount of DU in dry matter (in mg-DU/kg) for all compartments $C_{i}$ except the deep sediment sink and the export compartment $\left(Q_{i}\right)$, which were in mass per unit area (mg-DU/m $\left.{ }^{2}\right)$ removed from the system. Dose calculations, however, were based on the wet mass of tissue in order to account for the mass of water in tissue samples. Dose calculations are discussed in detail in Chapter 5 . Harvesting by humans and emigration were included in export (Baird and Ulanowicz 1989; Myers et al. 1993). The DU source in the model simulations was ambient water concentrations of $\mathrm{DU}\left(C_{0}\right.$, in milligrams of DU per liter, or mg-DU/L) in tidal creeks flowing from APG into Chesapeake Bay.

Our food-web model has the form of a first-order rate equation (Landrum et al. 1992). We calculated changes in the DU concentration in any compartment $C_{i}$ with each time step as the sum of all inputs from other compartments $C_{j}$ to $C_{i}$, minus all losses from $C_{i}$

$$
\frac{d C_{i}}{d t}=\sum\left(C_{j} \lambda_{i j}-C_{i} \lambda_{j i}\right)
$$

where $\lambda_{i j}$ is the rate of uptake (per day) by $C_{i}$ from $C_{j}$ and $\lambda_{j i}$ is the rate of loss (per day) from $C_{i}$ to $C_{j}$ (Myers et al. 1993). The inventory of DU at any given time is thus represented by the values of $C_{i}$ for each compartment at the time of interest. Inventories in compartments representing DU sinks, $Q_{11}$ (export) and $Q_{12}$ (deep sediment sink), were calculated in the same fashion. Most of the rate coefficients could not be obtained directly because they have not been estimated empirically; we derived them from the structural and functional characteristics of the aquatic food web and physiological properties of the compartment organisms. The structural characteristics are the relative abundance of species in different compartments. Functional characteristics are the rates of material flow between compartments. We 


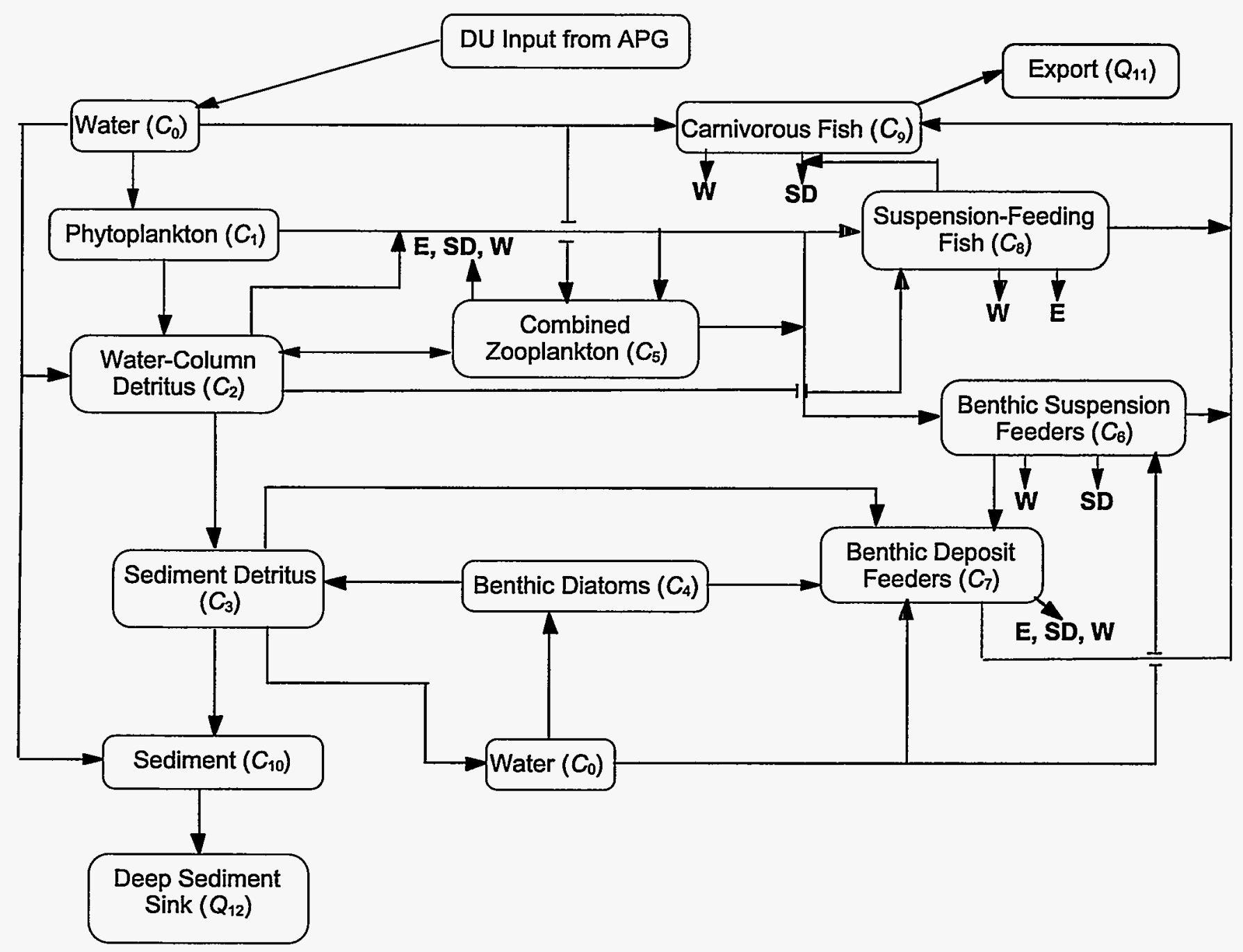

Figure 3-1. Conceptual model of uranium transport through the aquatic food web at APG based on the carbon-flux model of Baird and Ulanowicz (1989). Representative organisms for compartments (or feeding categories) are listed in Table 3-1. Arrows in the diagram indicate potential DU flow or transport to different compartments. Arrows to W, SD, and E represent losses from compartments to water, sediment detritus, and export compartments, respectively.

assumed that three compartments, $C_{1}, C_{2}$, and $C_{4}$ (Table 3-1), had DU transfer rates that were much more rapid than one day, primarily due to adsorptive processes. The model does not use different-sized time steps, so concentrations in these compartments were calculated using bioconcentration factors $B C F_{i}$, which were multiplied by $C_{0}$. 
Table 3-1. Ecological compartments of a carbon-flow model in the mesohaline region of Chesapeake Bay (Baird and Ulanowicz 1989) and corresponding compartments in the DU exposure model (Myers et al. 1993).

\begin{tabular}{|l|c|}
\hline \multicolumn{1}{|c|}{ Carbon Model Compartment } & $\begin{array}{c}\text { Exposure Model } \\
\text { Compartment } \\
\text { Identification }\end{array}$ \\
\hline Phytoplankton & $\mathrm{C}_{1}$ \\
Suspended Organic Matter & $\mathrm{C}_{2}$ \\
Sediment Organic Matter & $\mathrm{C}_{3}$ \\
Benthic Algae & $\mathrm{C}_{4}$ \\
Combined Zooplankton & $\mathrm{C}_{5}$ \\
Benthic Suspension Feeders & $\mathrm{C}_{6}$ \\
Benthic Deposit Feeders & $\mathrm{C}_{7}$ \\
Suspension Feeding Fish & $\mathrm{C}_{8}$ \\
Carnivorous Fish & $\mathrm{C}_{9}$ \\
\hline
\end{tabular}

\subsubsection{Derivation and Estimation of $\lambda_{i j}$}

DU transfer between compartments is represented conceptually by arrows in the box model (Figure 3-1), and the rate of transfer is described mathematically by $\lambda_{i j}$. Any compartment $j$ contained $C_{j} \mathrm{mg}-$ DU $/ \mathrm{kg}$, so the rate of increase in another compartment $C_{i}$ from any food source, $C_{j}$, was $C_{j} \lambda_{i j}$. The $\lambda_{i j}$ used to describe rates of DU transfer between model compartments is a function of physiological and ecological parameters such as compartment biomass $\beta_{i}\left(\mathrm{~kg}\right.$-dry matter $/ \mathrm{m}^{2}$ ), consumption rates $k_{i j}$ (per day), and DU assimilation rates $\alpha_{i j}$ (dimensionless). Of the 86 total input variables used in the model, $49 \%$ were obtained from the Chesapeake Bay model (Baird and Ulanowicz 1989) to describe food-web structure and function, and another $26 \%$ specifically describe DU transport processes or equilibrium concentrations (Mahon 1982; Trabalka and Garten 1983; Wrenn et al. 1985; Anderson et al. 1989a, $1989 \mathrm{~b})$. The remaining $\lambda_{i j}$ and their ranges were taken from published values for similar systems or species (Myers et al. 1993). Baird and Ulanowicz (1989) found that the structure and function of the mesohaline region of Chesapeake Bay did not change substantially from season to season, so we felt justified in using static structural and functional characteristics in each simulation. We scaled annual rates of carbon flow between model compartments (mg-C/m $\mathrm{m}^{2}-\mathrm{yr}^{-1}$; Baird and Ulanowicz 1989) so that daily rates integrated over a year were equal to annual flows in the carbon model and expressed this rate $k_{i j}$ as the daily fraction of prey biomass $\beta_{j}\left(\mathrm{~kg}-\mathrm{dry}\right.$ matter $/ \mathrm{m}^{2}$ ) consumed each day (Myers et al. 1993). Thus, $\beta_{j} k_{l j}$ represents the daily amount of biomass ingested $\left(\mathrm{kg}\right.$-dry matter $/ \mathrm{m}^{2}$-day $\left.{ }^{-1}\right)$ by organisms in the compartment described by $\mathrm{C}_{i}$. We used published mean, median, or modal values to set nominal values for input variables. Upper and lower values were estimated from published ranges or variances or assumed to be a constant proportion of nominal values (Myers et al. 1993). We used these values to analyze uncertainty in estimated DU concentrations caused by uncertainty in input variables. 
We allowed model compartments to accumulate DU from food and water ingestion only. Dermal exposure to DU from submersion in water or sediments was not included because DU concentrations were too low to make these exposure pathways significant relative to DU ingestion. Likewise, dermal exposure due to particles adsorbed to organisms comprising the compartments resulted in insignificant doses to the organisms compared with ingested DU.

A fraction of total DU ingested with food was assimilated, $\alpha_{i j}$, (Wrenn et al. 1985) so that the DU transfer rate $\lambda_{i j}$ (per day) to compartment $i$ from compartment $j$ was

$$
\lambda_{i j}=\frac{\beta_{j} k_{i j} \alpha_{i j}}{\beta_{i}},
$$

where $\beta_{i}\left(\mathrm{~kg}\right.$-dry matter $\left./ \mathrm{m}^{2}\right)$ is standing crop biomass of the consumer (Baird and Ulanowicz 1989). The rate parameters for uptake from water, $\lambda_{i 0}\left(\mathrm{~L} / \mathrm{kg}^{\left.-\mathrm{day}^{-1}\right)}\right.$ for $C_{5}$ through $C_{9}$ were calculated as:

$$
\lambda_{i 0}=\frac{k_{i 0} \alpha_{i 0 j}}{\beta_{i}},
$$

where $k_{i 0}$ is in $\mathrm{L} / \mathrm{m}^{2}$-day ${ }^{-1}$ and other parameters are as already defined. We derived $k_{i 0}$ for $C_{7}-C_{9}$ using the relationship

$$
k_{i 0}=\frac{v_{i} \beta_{i} \gamma_{i}}{\delta_{i}},
$$

where $v_{i}$ is the volume of water passing over the gill (L/day- $\mathrm{kg}^{-1}$ of fresh mass) (Langille et al. 1983), $\gamma_{i}$ is the parameter to scale ventilation volume to compartment-specific metabolic rates, and $\delta_{i}$ converts $\beta_{i}$ to fresh mass (Jørgenson 1979). For any $C_{i}$, the loss rate of DU from $C_{i}$ to $C_{j}$ due to feeding was calculated as $-\lambda_{j i} C_{i}$.

We used the steady-state, average annual biomass values from the Baird and Ulanowicz model (Baird and Ulanowicz 1989) for our nominal $\beta_{i}$ (Myers et al. 1993). A constant biomass structure causes DU concentrations to be diluted as growth and recruitment of new biomass replace that lost through mortality processes. Many physiological processes are related to body size and metabolic rates of organisms (Calder 1984), including contaminant elimination rates (Newman and Heagler 1991). For example, we calculated DU elimination $\left(\lambda_{0, i}\right)$ from compartments $\mathrm{C}_{5}-\mathrm{C}_{9}$ to water at rates that were a multiple $\left(\tau_{i}\right)$ of respiration rate, $\rho_{i}$ (per day) (Myers et al. 1993). These rates were calculated as

$$
\lambda_{0, i}=\frac{\rho_{i}}{\tau_{i}}
$$

\subsection{Model Uncertainty and Sensitivity Analyses}

Investigations of relationships between input variables and output from exposure and risk assessment models can be divided into analyses of uncertainty and sensitivity. The goal of uncertainty analysis is to estimate the uncertainty in the computed DU concentrations by incorporating into the computations the uncertainty from the input variables. Input variables in these model equations represent abiotic or biotic 
parameters that are known with varying degrees of certainty. When the input values are uncertain, the calculated model outputs are also uncertain. One way to characterize uncertainty of input variables is with a lower bound, an upper bound, and a nominal value. The nominal value may in some sense represent the most likely value for the input variable, whereas the range represents the possible values of input variables. The uncertainty in model output depends on the variability in these input variables.

There are two basic perspectives for uncertainty analyses. One may assume that there is a true value for each input variable and that the ranges and nominal values of each do not represent any notion of probability. In this case, the uncertainty in the output variable is viewed as the maximum and minimum values the output variable can attain over the range of input variables. From a practical standpoint, the maximum and minimum values of the output variables are impossible to compute for all but the smallest number of input variables. For example, if there are 84 input variables and if it is known that the output variable is a monotonic function of the inputs (the direction unknown), then it would take more than $10^{25}$ computer runs to determine the maximum and minimum values of the output variables. Given the impossibility of this task even with an efficient search algorithm, we used the second method of uncertainty analysis, which is to assume that a probability distribution characterizes the uncertainty in the input parameters.

Assuming that the input parameters are characterized by a probability distribution is not unusual. Probability distributions are subjective evaluations of parameters (Savage 1954; Lindley 1965). Without this probabilistic paradigm, the nominal value of an input variable has no clear meaning. With the probabalistic paradigm, however, the nominal value is considered the "most likely" value of the parameter (e.g., the mean or median value).

In contrast to uncertainty analysis, sensitivity analysis involves the study of the uncertainty bounds of model output to determine which parameters contribute the most to these bounds. There are numerous methods for conducting sensitivity analyses (Inman and Helton 1988; Helton and Inman 1982). We used a derivative method similar to that of Ronen (1988), in which the variance of a function of $n$ variables, $f\left(v_{1}, v_{2}, \ldots, v_{n}\right)$ is approximated by

$$
\operatorname{Var}(f) \approx \sum_{i}^{n}\left(\frac{\delta f}{\delta v_{i}}\right) \sigma_{i}^{2},
$$

where $\sigma_{l}^{2}$ is the variance of the variable, $v_{i}$. The range (upper bound minus the lower bound) of each individual input variable is an approximation of its variance. Therefore, the individual components of $\left|\partial f / \partial v_{i}\right| R_{i}$ are sensitivity measures, where $R_{i}$ is the range of the $i$ th input variable $v_{i}$. The values of $\left|\partial f / \partial v_{i}\right|$ are computed by numerical derivatives for each of the input variables.

We used two distributions in our uncertainty and sensitivity analyses. First, a uniform distribution was assumed across the range of the input variables. In this case the nominal value is discarded, and any value in the range of input values is equally likely. Our second approach was to use the nominal value and assume that the uncertainty in an input variable can be represented by a probability distribution that has the nominal value as its mean value and is unimodal like a normal or Gaussian distribution. The family of beta distributions can take on a variety of shapes that describe the uncertainty in the parameter of interest 
and are defined within the range of values for the parameter. Shapes of beta distributions include the uniform distribution as a special case of the unimodal distributions that can be symmetric (e.g., a bell curve) or skewed and not symmetric.

The probability density functions used here are represented by

$$
f(x) \propto(x-L)^{(p-1)}(U-x)^{(q-1)},
$$

where $L$ is the lower limit of the range and $U$ is the upper limit. The constants $p$ and $q$ fix the mean of the distribution at the nominal value, make the distribution unimodal, and ensure the distribution has a large variance. The variance of the distribution is made large to reflect the fact that the range of the input parameter along with its nominal value determines the uncertainty in the parameter.

For the distribution to be unimodal, both $p$ and $q$ must be larger than 1 . The expected, or mean, value of the beta distribution is

$$
E(x)=\left(\frac{p}{p+q}\right)(\mathrm{U}-\mathrm{L})+L .
$$

In order to maintain the desired shape of the beta distribution, both $p$ and $q$ are greater than 1 . The variance of the distribution is maximized if either $p$ or $q$ is close to 1 . Therefore, we set one of these parameters equal to 1.1 to reflect the large and realistic uncertainly in the input parameters. Values greater than 1.1 would maintain the shape of the distribution but would unrealistically reduce uncertainty; conversely, values less than 1.1 but still greater than 1 would increase uncertainty more than necessary. The beta distributions are skewed toward the lower bound $L$ if $p>q$ and toward the upper bound $U$ if $p<$ $q$. The distribution is symmetric for $p=q$. We skewed the distribution toward the lower bound if the nominal value was closer to the lower bound than to the upper bound. We skewed it toward the upper bound if the opposite was true.

If the nominal value $N$ is closer to $L$ than to $U$,

$$
p=1.1\left(\frac{N-L}{U-N}\right)
$$

and $q=1.1$; otherwise, $p=1.1$ and

$$
q=1.1\left(\frac{U-N}{N-L}\right)
$$

Regardless of the distribution used (uniform or beta distribution), the variance (i.e., the uncertainty) in the output $Y_{i}$ can be approximated using a Taylor series of the form

$$
\operatorname{Var}\left(Y_{i}\right)=\operatorname{Var}(h(\mathbf{X})) \approx \sum_{j=1}^{n}\left(\frac{\partial h}{\partial x_{j}}\right)^{2} \sigma_{x_{j}}^{2}
$$

where $\mathbf{X}$ is a vector of input variables, $x_{j}$ is the $j$ th input variable and $\sigma_{x j}^{2}$ is the variance of $x_{j}$ (Ronen 1988). Each input variable therefore contributes 


$$
\left(\frac{\partial h}{\partial x_{j}}\right)^{2} \sigma_{x_{j}}^{2}
$$

to the uncertainty in the output variable. So,

$$
\left|\left(\frac{\partial h}{\partial x_{j}}\right)\right| \sigma_{x_{j}}
$$

is used as a sensitivity measure for the $j$ th input variable.

The variance $\sigma_{x_{j}}^{2}$ for the uniform distributions was

$$
\sigma_{x_{j}}^{2}=\frac{(U-L)^{2}}{12}
$$

and

$$
\sigma_{x_{j}}^{2}=\frac{(U-L)^{2} p q}{(p+q)^{2}(p+q+1)}
$$

for the beta distributions.

For the uncertainty analyses, one-thousand 5000-day-long simulations were performed. For each simulation, values of the input variables were chosen by Latin hypercube sampling (LHS) from the uniform or beta probability distributions (McKay et al. 1979). LHS forces input variables to be drawn from the full range of possible values by dividing the range into $n$ subranges of equal probability from which random values are drawn. For each output variable, summary statistics, such as the mean, standard deviation, and percentiles, were compiled.

For the sensitivity analyses, numeric derivatives were computed by perturbing each parameter at its nominal value. We constructed plots of sensitivity measures for each combination of input and output variables and graphically assessed the importance of each input variable on DU concentrations. We collected input variable sensitivity measures (Equation 3-13) from an arbitrary time step (day 3000), squared these values, and used the square root of the sum of these measures to evaluate the relative contributions to output uncertainty by different food-web compartments and parameter categories. Model input parameters were divided into those that described the uranium source, physiological processes, structural and functional characteristics of the aquatic food web, and abiotic properties and processes. Physiological parameters described interactions of individual organisms with DU, such as DU assimilation and elimination rates. Structural parameters described the state of the system, such as the standing crop biomass of each compartment. Functional parameters described rates of change of the system on an ecological scale, such as feeding rates (Bartell et al. 1992). We used 3 bioconcentration factors in the model, 4 input variables classified into the abiotic transport category, 10 structural variables, 28 functional variables, and 39 physiological variables for the beta distributions. 


\subsection{Results of Modeling}

\subsubsection{Model Uncertainty and Sensitivity Analyses-Aquatic Model}

\subsubsection{Uniform Input Distributions}

DU concentrations calculated for model compartments were highly sensitive to the water concentrations (Table 3-2) used to drive the model. Input water concentrations ranged from $3 \times 10^{-4} \mathrm{mg} / \mathrm{L}$ to $3 \mathrm{mg} / \mathrm{L}$, and uncertainty in this input variable dominated all others. If water concentration was exactly known, then no more than 2 parameters per compartment accounted for nearly $95 \%$ of the uncertainty in DU concentrations in zooplankton, fish, and benthic organisms $\left(C_{5}-C_{9}\right)$. The most influential food-web parameter derived from uniform input distributions was the assimilation coefficient for uptake from water $\alpha_{i, 0}$ (Table 3-2), which averaged 78\% (range: 62\%-86\%) of total uncertainty in consumer compartments when source effects were excluded. The next most important parameter, $\tau_{i}$, scaled DU elimination rates to compartment metabolic rates. It averaged about $16 \%(8 \%-32 \%)$ of the uncertainty when the effects of water concentration were eliminated.

Total uncertainty, as estimated by the sum of the squared sensitivity measures (Equations 3-11 and 313) relative to each compartment, was greatest for carnivorous fish. The order of relative uncertainty (from greatest to least) for other compartments was suspension feeding fish, zooplankton, benthic diatoms and phytoplankton, benthic deposit feeders, and benthic suspension feeders (Figure 3-2a). We calculated the DU concentrations in phytoplankton $\left(C_{1}\right)$ and benthic diatoms $\left(C_{4}\right)$ using equilibrium $B C F \mathrm{~s} ; C_{1}$ and $C_{4}$ were large contributors to overall uncertainty in the exposure model (Figure 3-2b). Physiological model parameters used to predict DU uptake and elimination contributed the most to uncertainty in DU concentrations, largely due to uncertainty in DU assimiliation rates from water (Table 3-2). DU elimination rates and ventilation rates also contributed significantly to the uncertainty. Structural and functional aspects of the aquatic food web and abiotic transport parameters had small effects on overall model uncertainty (Figure 3-2b).

\subsubsection{Beta Input Distributions}

When input water concentrations were drawn from a beta distribution having a nominal value of $0.003 \mathrm{mg} / \mathrm{L}$ and variance scaled from $3 \times 10^{-4} \mathrm{mg} / \mathrm{L}$ to $3 \mathrm{mg} / \mathrm{L}$, large values were much less likely and the sensitivity of the output to DU concentrations was reduced accordingly (Table 3-2). Water concentration was a major contributor to uncertainty in DU concentrations for only three compartments $\left(C_{1}, C_{2}\right.$, and $\left.C_{4}\right)$ when values were selected from the beta distribution. Water concentrations derived from beta distributions accounted for an average of $2 \%(0.6 \%-2.7 \%)$ of the uncertainty in DU concentrations for the other five biotic compartments $\left(C_{5}-C_{9}\right)$ and $2 \%$ of the uncertainty in sediment detritus concentrations $\left(C_{3}\right)$. Forty-four percent of the variation in sediment concentrations was attributed to variation in the concentration of $\mathrm{DU}$ in water. Fractional assimilation of $\mathrm{DU}$ from water by organisms $\left(\alpha_{i, 0}\right)$ also influenced output uncertainty when beta probability distributions were used to propagate uncertainty (Table 3-2). An average of $61 \%$ of output uncertainty for the zooplankton, fish, and benthic organism compartments $\left(C_{5}-C_{9}\right)$ was due to $\alpha_{i, 0}$. Compartment biomass $\beta_{i}$ was more influential than $\alpha_{i, 0}$ for the 
Table 3-2. Relative sensitivities of calculated DU concentrations $\left(C_{i}\right.$ in $\left.\mathrm{mg} / \mathrm{kg}\right)$ and inventories $\left(Q_{i}\right.$ in $\mathrm{mg} / \mathrm{m}^{2}$ ) to exposure model input variables when input values were drawn from uniform and beta probability distributions. Appendix A is a list of input values for all parameters.

\begin{tabular}{|c|c|c|c|}
\hline \multirow[b]{2}{*}{$\begin{array}{c}\text { Compartment } \\
\text { ID }^{\mathrm{b}}\end{array}$} & \multicolumn{3}{|c|}{ Relative Sensitivities of Input Variables ${ }^{2}$} \\
\hline & Major & Intermediate & Minor \\
\hline \multicolumn{4}{|c|}{ Uniform Distributions } \\
\hline $\mathrm{C}_{1}$ & $C_{0}$ & $B C F_{1}$ & \\
\hline $\mathrm{C}_{2}$ & $C_{0}$ & $B C F_{2}$ & \\
\hline $\mathrm{C}_{3}$ & $C_{0}$ & $\alpha_{7,0}, B C F_{2}$ & $k_{10,3}, \tau_{7}$ \\
\hline $\mathrm{C}_{4}$ & $C_{0}$ & $B C F_{4}$ & \\
\hline $\mathrm{C}_{5}$ & $C_{0}$ & $\alpha_{5,0}$ & $\tau_{5}, k_{5,0}$ \\
\hline $\mathrm{C}_{6}$ & $C_{0}$ & $\alpha_{6,0}$ & $\tau_{6}$ \\
\hline $\mathrm{C}_{7}$ & $C_{0}$ & $\alpha_{7,0}$ & $\tau_{7}$ \\
\hline $\mathrm{C}_{8}$ & $C_{0}$ & $\alpha_{8,0}$ & $\tau_{8}$ \\
\hline $\mathrm{C}_{9}$ & $C_{0}$ & $\alpha_{9,0}$ & $\tau_{9}$ \\
\hline $\mathrm{C}_{10}$ & $C_{0}$ & $\beta_{10}, k_{10,0}$ & \\
\hline $\mathrm{Q}_{11}$ & $C_{0}$ & $\alpha_{5,0}, \tau_{5}$ & $k_{5,0}$ \\
\hline $\mathrm{Q}_{12}$ & $C_{0}$ & $k_{12,10}, k_{10,0}$ & \\
\hline \multicolumn{4}{|c|}{ Beta Distributions } \\
\hline $\mathrm{C}_{1}$ & $C_{0}$ & $B C F_{1}$ & \\
\hline $\mathrm{C}_{2}$ & $C_{0}$ & & \\
\hline $\mathrm{C}_{3}$ & $k_{10,3}$ & $\alpha_{7,0}, \beta_{7}, k_{7,3}, k_{3,2}$ & $C_{0}, \beta_{9}, \delta_{7}, \rho_{7}, \beta_{8}, \beta_{3}$ \\
\hline $\mathrm{C}_{4}$ & $C_{0}$ & $B C F_{4}$ & \\
\hline $\mathrm{C}_{5}$ & $\beta_{5}$ & $\alpha_{5,0}$ & $C_{0}, \rho_{5}$ \\
\hline $\mathrm{C}_{6}$ & $\beta_{6}, \alpha_{6,0}$ & $\rho_{6}, k_{3,6}, k_{11,6}$ & $C_{0}, k_{6,1}, k_{6,0}, \alpha_{6,1}, k_{7,6}$, \\
\hline $\mathrm{C}_{7}$ & $\alpha_{7,0}$ & & $k_{3,7}, C_{0}, \delta_{7}, \rho_{7}, \gamma_{7}$ \\
\hline $\mathrm{C}_{8}$ & $\alpha_{8,0}$ & $k_{3,8}$ & $\rho_{8}, C_{0}, \delta_{8}, k_{9,8}, k_{11,8}, \beta_{8}$ \\
\hline $\mathrm{C}_{9}$ & $\alpha_{9,0}$ & $k_{3,9}$ & $\rho_{9}, k_{11,9}, C_{0}, \delta_{9}, \gamma_{9}$ \\
\hline $\mathrm{C}_{10}$ & $k_{12,10}$ & $C_{0}$ & $k_{10,0}$ \\
\hline $\mathrm{Q}_{11}$ & $\alpha_{5,0}$ & $k_{11,5}$ & $C_{0}, k_{11,7}, \beta_{9}$ \\
\hline $\mathrm{Q}_{12}$ & $k_{12,10}$ & & $C_{o}$ \\
\hline
\end{tabular}

${ }^{2}$ Inputs are listed in order of descending sensitivity values within each compartment and category.

${ }^{b}$ Compartment IDs are explained in Table 3-1 except for $C_{10}$ (surface sediments), $Q_{11}$ (quantity exported from the system), and $Q_{12}$ (quantity in the deep sediment sink). 


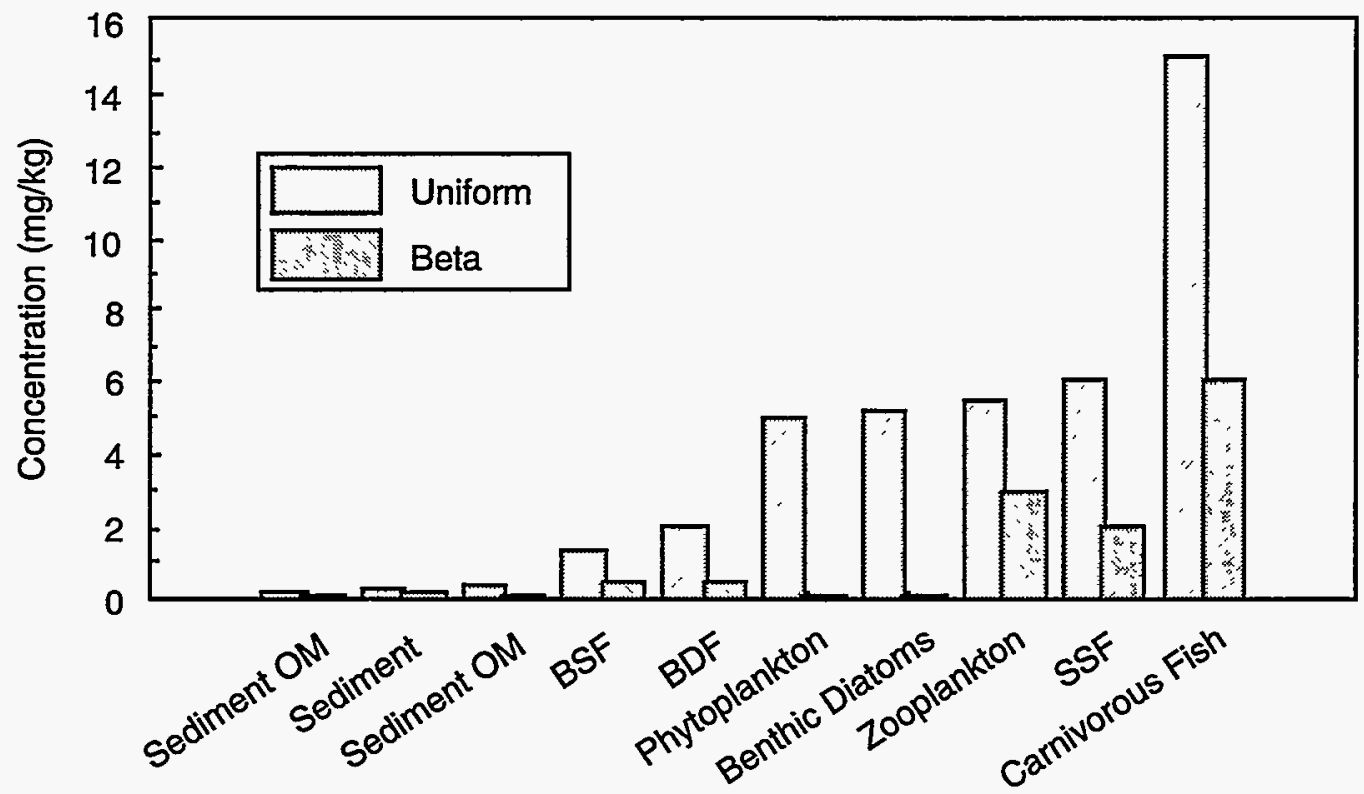

Figure 3-2a. Relative uncertainty of exposure model compartments measured by the square root of the sum of the squared input variable sensitivity values. Input values were generated from uniform and beta distributions as indicated. OM is organic matter, BSF is benthic suspension feeders, BDF is benthic deposit feeders, and SFF is suspension feeding fish.

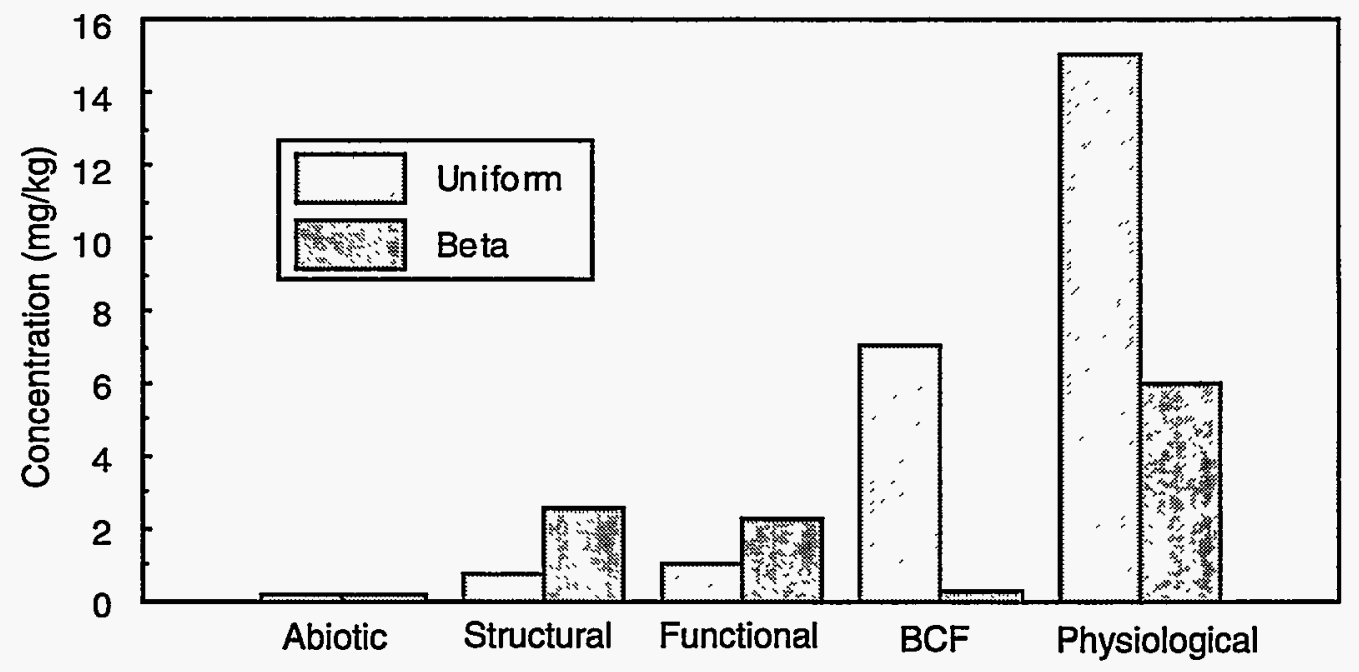

Figure 3-2b. Relative uncertainty of compartments when input values were aggregated by their roles in the exposure model. Abiotic parameters describe transport between abiotic compartments, structural parameters describe the standing crop biomass structure of the food web, functional parameters describe the rates of material exchange between compartments, BCFs were bioconcentration factors used to calculate compartment concentrations at equilibrium, and physiological parameters include compartment ventilation rates, elimination rates, and DU assimilation rates. Uncertainty due to water concentration was not included. 
zooplankton (78\% of the uncertainty) and shellfish (44\% of the uncertainty) compartments. Compartment biomass determined the dilution of DU in each compartment and helped set consumption, ventilation, and elimination rates. Decomposition rate $\left(k_{10,3}\right)$ for sediment detritus $\left(\mathrm{C}_{3}\right)$ most strongly influenced DU concentrations in this compartment.

Overall, imposing a "most likely" nominal value on each range of input values caused model uncertainty to be much less than when uncertainty was estimated from uniform distributions. Uncertainty measures based on uniform distributions and aggregated over food-web compartments $C_{1}-C_{9}$ were more than $3 \times 10^{5}$ times greater than the same measures based on beta distributions. Most of the uncertainty was due to water concentrations. When uncertainty due to variation in water concentration was removed from the analysis, uncertainty due to variations in the parameters of the different food-web compartments was about seven times greater when drawn from uniform distributions than from beta input distributions. Exposure model compartments did not have the same rank order of uncertainty as when uniform input distributions were used (Figure 3-2a). Physiological parameters also were a dominant source of uncertainty when beta input distributions were used, but the importance of bioconcentration factors was reduced. Structural characteristics of the food web contributed $18 \%$ of the food-web uncertainty and functional characteristics accounted for 13\% (Figure 3-2b).

\subsubsection{Predicted DU Concentrations}

The amounts of DU in the phytoplankton and benthic diatom compartments were calculated based on bioconcentration factors and had the largest estimated nominal concentrations of $2.6 \mathrm{mg} / \mathrm{kg}$. Carnivorous fish had an estimated nominal concentration of $1.7 \mathrm{mg} / \mathrm{kg}$. Estimated nominal DU levels were $0.5-1.0 \mathrm{mg} / \mathrm{kg}$ for zooplankton, suspension feeding fish, and sediments; $0.5-1.0 \mathrm{mg} / \mathrm{kg}$ in suspended detritus, benthic deposit feeders, and benthic suspension feeders; and $0.05 \mathrm{mg} / \mathrm{kg}$ in sediment detritus. The nominal water concentration was $0.003 \mathrm{mg} / \mathrm{L}$, although concentrations as low as $3 \times 10^{-4} \mathrm{mg} / \mathrm{L}$ and as high as $3 \mathrm{mg} / \mathrm{L}$ were allowed as model inputs. The range of water concentrations was based on the distribution of $U$ in a nationwide study of $U$ in water samples from wells and surface sources (Cothern and Lappenbusch 1983). The range is extremely conservative but incorporates $U$ concentrations that could result from natural $U$ or $D U$ from penetrators in groundwater. With a uniform probability distribution of water concentrations the expected value for the mean is the midpoint of the range (that is, $1.5 \mathrm{mg} / \mathrm{L}$ ). When we sampled input water concentrations from a beta distribution based on these values, the mean input value was $0.0045 \mathrm{mg} / \mathrm{L}$, although extreme values were possible (Table 3-3). The large range of input water concentrations plus uncertainty associated with other model parameter values caused equilibrium output concentrations calculated for compartments to cover several orders of magnitude (Table 3-3). Values between the 25th and 75th percentiles give approximations of likely values from this exposure model and of the beta distributions assigned to model inputs. At the 75th percentile, concentrations of DU were about $3.5 \mathrm{mg} / \mathrm{kg}$ in phytoplankton and benthic diatoms, about $2 \mathrm{mg} / \mathrm{kg}$ in carnivorous fish, and $<1 \mathrm{mg} / \mathrm{kg}$ in all other compartments. Median values for biotic compartments were all greater than the median water concentration. DU did not concentrate in any compartment greater than two to three orders of magnitude more than in water. 
Table 3-3. DU concentrations calculated from aquatic exposure model. Inputs drawn from beta distributions. All values are in $\mathrm{mg} / \mathrm{kg}$.

\begin{tabular}{|l|c|c|c|c|}
\hline \multicolumn{1}{|c|}{ Compartment Name } & Mean & Median & Range & 25th to 75th Percentiles \\
\hline Water & 0.008 & 0.004 & 0.0005 to 2 & 0.002 to 0.008 \\
Phytoplankton & 4 & 2 & 0.01 to 1000 & 0.9 to 3.5 \\
Suspended OM & 0.2 & 0.08 & 0.001 to 100 & 0.04 to 0.1 \\
Sediment OM & 0.08 & 0.04 & 0.0005 to 10 & 0.01 to 0.1 \\
Benthic Diatoms & 4 & 2 & 0.01 to 1000 & 1 to 3.5 \\
Zooplankton & 1 & 0.4 & 0.004 to 1000 & 0.1 to 0.8 \\
Benthic Sediment Feeders & 0.5 & 0.1 & 0.001 to 100 & 0.08 to 0.4 \\
Benthic Deposit Feeders & 0.5 & 0.2 & 0.001 to 40 & 0.09 to 0.5 \\
Suspension Feeding Fish & 1 & 0.8 & 0.006 to 100 & 0.1 to 1 \\
Carnivorous Fish & 4 & 1 & 0.006 to 1000 & 0.8 to 2 \\
\hline
\end{tabular}

\subsection{Discussion}

\subsubsection{Model Uncertainty and Sensitivity Analyses}

\subsubsection{Uncertainty and Sensitivity Analysis Methodology}

The sensitivity analysis we used is one of many possible techniques to investigate the behavior of response variables (in our case, predicted DU concentrations) as they relate to the input parameters of a model. Computing the derivatives is the simplest of all sensitivity methods; the number of computer runs required is at most only twice the number of parameters, and these methods allow for combining the sensitivities of various parameters by adding together some of the individual terms from Equation 3-12. However, these sensitivity measures give information about the derivatives only at one point (here the nominal value of the parameter) in a multidimensional space. Other methodologies compute the standard deviation and the variance of the derivatives at randomly selected positions in the multidimensional parameter space (Morris 1991), compute regressions or partial correlation coefficients (Inman et al. 1981a, 1981b), calculate variance decompositions (McKay 1992), or find directions of reduced dimensionality in the parameter space $(\mathrm{Li} 1991,1992)$. The number of computer runs required for each of these methodologies is about 10 times the number of parameters. Since the code used here ran in a short amount of time, each of these alternative methodologies was implemented and computed. The alternative methodologies identified the same most-sensitive parameters for this model as our analysis using uniform input distributions (R.J. Beckman, unpublished data).

\subsubsection{Input Distributions, Sensitivities, and Output Uncertainty}

The range of water concentrations dominated all other sources of uncertainty generated using uniform input distributions. Uncertainty projections using uniform input distributions were unacceptably high because this approach ignored information about biotic and abiotic processes. When uncertainty due to 
the source term was eliminated, overall model uncertainty was still greater when uniform distributions were used instead of beta distributions because of the inefficient use of available information. When information on likely nominal values and probability distributions was included, predicted uncertainty about DU concentrations decreased. Although we could have used normal, lognormal, or other probability distributions to generate input variable distributions, we used beta distributions because of their flexibility and because we often lacked the information necessary to specify other distributions. We scaled beta input distributions with the ranges of input variables and estimates of central tendency so that values selected for input variables were more likely to be nearer to the nominal value than to the upper and lower bounds of their ranges. These distributions also had large variances to reflect our uncertainty about the exact parameter distributions. Better descriptions of ecological exposure model rate equations are likely to further reduce uncertainty by reducing variance in predicted exposures, as well as by reducing bias in predicted DU concentrations.

Physiological, structural, and functional parameters caused the most uncertainty in predictions of the exposure model based on beta distributions because DU transport through the aquatic food web was mainly a function of these three parameter groups. Physiological parameters determined rates of DU assimilation and elimination by organisms. In the case of trophic interactions, structural parameters governed which organisms interacted in the model ecosystem, and functional parameters controlled the rates of these interactions. The nature of how these parameters interact to control and limit organisms has long been a topic of disagreement (see Arditi and Saïah 1992; Berryman 1992; Ginzburg and Akçakaya 1992; Gutierrez 1992; Hunter and Price 1992; Mattson and Berryman 1992; Mattson and Hunter 1992; Menge 1992; Power 1992; Strong 1992; and Slobodkin 1992 for recent reviews). Therefore, the same factors that are important in setting food-web dynamics are important determinants of DU transport in food webs.

\subsubsection{Predicted DU Concentrations}

We calculated equilibrium compartment concentrations using nominal input values, and the calculated values were within an order of magnitude of reported uranium concentrations in the respective trophic levels. The model predicted that zooplankton $\left(C_{5}\right)$ would accumulate DU an average of $175-350$ times the ambient water concentrations, compared with bioconcentration factors of about 350 for marine amphipods (Ahsanullah and Williams 1986). Predicted DU concentrations in shellfish $\left(C_{6}\right)$ and other benthic organisms $\left(C_{7}\right)$ were 50-75 times the DU concentration in water (Myers et al. 1993), compared with $U$ bioconcentration factors of between 4 and 18 for benthic organisms (Ahsanullah and Williams 1989). At environmental U concentrations similar to our nominal water value, wild trout had bioconcentration factors as high as 140-260 (Nichols and Scholz 1989), and experimentally exposed trout accumulated between 2 and 40 times the concentration of $U$ in water (Poston 1982; 1983). Although trout are not found at APG, the data from the trout studies were used because no other data were available for fish found at APG. Using these data, fish compartments in the model accumulated 230-554 times nominal water concentrations. 


\subsubsection{A Strategy for Reducing Uncertainty of Predicted Exposures}

Modeling approaches are necessary for producing probabilistic estimates of ecological exposure and risk (Barnthouse 1992), and ecological exposure models based on literature values from surrogate ecosystems can provide a valuable starting point. However, knowledge about transport processes in these models will be imperfect and will cause uncertainty in predictions. Uncertainty and sensitivity analyses of exposure models have provided insight into sources of uncertainty in model predictions. For example, Breshears et al. (1992) conducted variance-based sensitivity analyses of the PATHWAY terrestrial foodchain model (Whicker and Kirchner 1987) to identify input parameters that were most influential in determining human ingestion of ${ }^{131} \mathrm{I}$ and ${ }^{137} \mathrm{Cs}$. Parameters such as foliar deposition rates and radionuclide resuspension factors significantly influenced model outputs, and further study of these processes were encouraged to reduce uncertainty in model predictions. Instead of performing uncertainty/sensitivity analyses when a final exposure model was assembled, we constructed and analyzed a preliminary model (set of working hypotheses) about DU transport at APG. We used uncertainty and sensitivity analyses to identify potentially important pathways in the model so that relevant experiments and field sampling programs could be designed to test these hypotheses.

We identified the obvious result that the source of DU exposure in our model was the single most important piece of information needed to estimate concentrations in biota. When a likely probability distribution was assumed for water concentrations, other uptake processes from water to organisms influenced the DU concentrations in the food web. DU concentrations also were sensitive to flows of DU between compartments due to elimination, export from the system, and return to the sediment detritus pool. With this information, research aimed at reducing uncertainty about these pathways and other transport processes can be planned and implemented. Any reduction in uncertainty about DU transport processes and parameters will reduce uncertainty about predicted DU concentrations in aquatic life at APG (Figure 3-3). Important transport pathways will have $\lambda_{i j}$ values that are significantly greater than zero when estimated from laboratory or field experiments. For example, laboratory studies could be conducted to estimate time-dependent concentrations of DU in phytoplankton, zooplankton, benthic deposit feeders, and carnivorous fish from the water pathway and to estimate the sediment-to-water sorption/desorption parameters (Figure 3-3). In addition, measurements of site-specific uptake kinetics in benthic suspension feeders and benthic deposit feeders from field exposures or on-site collections could be used to modify the food-web structure from that in the Chesapeake Bay model (Baird and Ulanowicz 1989) to that at APG. For example, Bartell et al. (1992) showed that the most important factor controlling the quality of their site-specific predictions was knowledge of the site-specific food-web structure, followed by the quality of exposure concentration estimates. Our analyses did not evaluate uncertainty resulting from inadequate model structure, but the model does provide a framework for studies to evaluate the appropriateness of the structure. Our site is north of the mesohaline region of Chesapeake Bay, upon which the model is based, and it has a different suite of interacting species. Thorough field surveys can provide information on food-web structural components. During model development we recognized that our adaptation of the Chesapeake Bay model (Baird and Ulanowicz 1989) does not adequately account for DU uptake from sediments and sediment pore-water or by transdermal absorption 


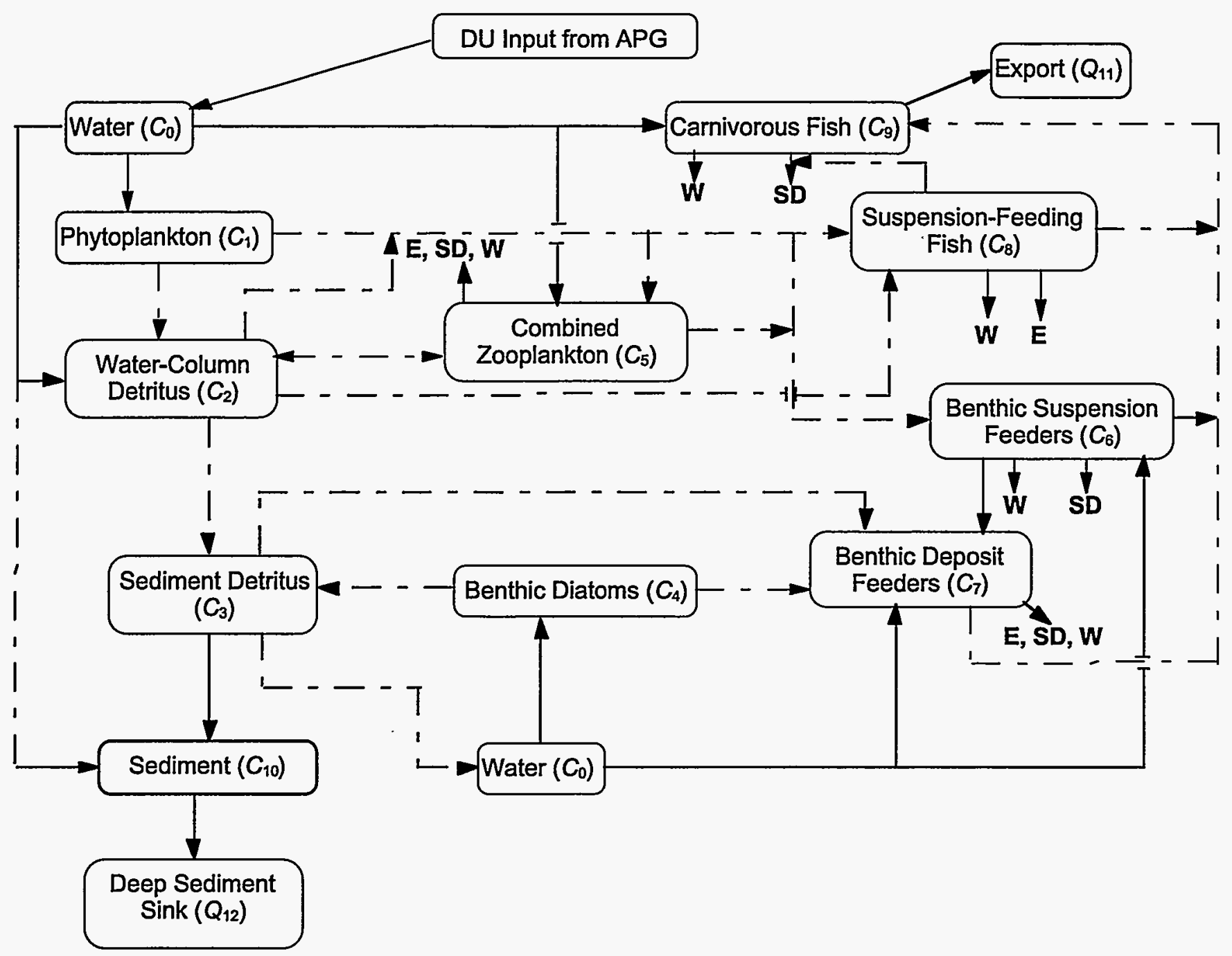

Figure 3-3. DU transport pathways identified from sensitivity analyses. Solid lines indicate major importance to model output, and dashed lines indicate intermediate to minor importance.

(Yuile 1973). Therefore, relevant laboratory experiments to explore these processes and to estimate their parameters would be needed to reduce uncertainty in these DU transport pathways.

Laboratory and field experiments can generate input parameter values and probability distributions for refining preliminary exposure models. Comparison of a revised model with DU concentrations in aquatic organisms from APG can give an assessment of the quality of model predictions. The relative fit of predictions from each model version also documents the change in uncertainty produced by each set of experiments (Bartell et al. 1992). Additional iterations of sensitivity/uncertainty analysis, experimentation, and model revision can be performed until sufficient information is assembled to predict exposures to within acceptable levels of uncertainty.

Complex ecosystem exposure models may not be the most efficient models for making long-term predictions about ecological impacts. Data to select a best model from competing models, however, are 
often inadequate (Barnthouse et al. 1984). The primary function of the model used for APG was to provide a framework for collecting data that could be used to reject the original model in favor of a better one, not to predict accurate DU concentrations in aquatic organisms. An alternative model having sitespecific structural and functional characteristics and parameterized with site-specific data would better predict DU concentrations in aquatic organisms at APG. Our uncertainty and sensitivity analyses helped to identify data needed to construct such a model. Thus, by focusing attention and resources on parameters and processes that most contribute to uncertainty in the modeling scenarios, efficient reduction of uncertainty about ecological exposures may be achieved.

\subsection{Steady-State Model, APG Terrestrial Ecosystem}

\subsubsection{Model Structure}

The steady-state model was based on a two-component model described elsewhere (NCRP 1984a). The National Council on Radiation Protection and Measurements (NCRP) model was developed for estimating radionuclide uptake by plants and animals in the vicinity of nuclear power plants and includes intake by animals of radionuclides from foliar surfaces and radionuclides internally deposited in the forage plants, as well as those ingested from drinking water and contaminated soil. Inhalation of radionuclides was not included in the model shown in Figure 3-4 because initial calculations showed that inhalation is an insignificant exposure pathway compared with the other pathways. Airborne DU is concentrated on foliar surfaces through resuspension and contributes approximately two orders of magnitude more radiological dose than inhalation of DU-containing dust in ambient air.

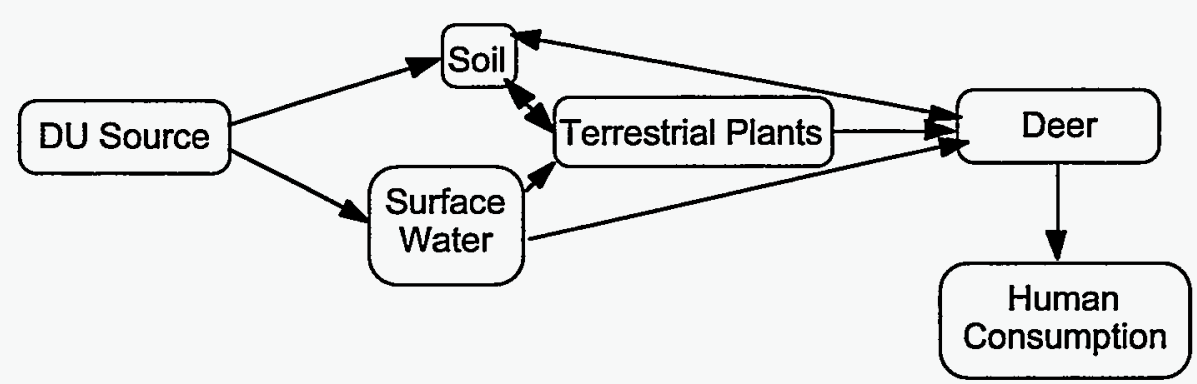

Figure 3-4. Schematic of the steady-state model. Model shows DU transfer to deer from drinking water, soil ingestion, and plant ingestion pathways and transfer to humans from deer tissue consumption.

The NCRP model was modified to estimate the concentration of $U$ or DU in deer tissue by including a term for soil ingestion by animals, changing the animal of interest from cattle to deer and modifying the food and water intake rates accordingly, and including the transfer of DU to man by way of consumption of deer tissue (Arthur and Gates 1988; Lautier et al. 1988; Alldredge 1974; Arthur and Alldredge 1979). Figure 3-4 shows a schematic of the steady-state model. The modified NCRP model was tested in a risk 
assessment of DU fragments at Jefferson Proving Ground (JPG) and was shown to provide results similar to other risk assessment models (Ebinger and Hansen 1994a).

DU transfer between system compartments is quantified as

$$
C_{i}=F_{\mathrm{a}}\left[\left(C_{\mathrm{a}}+C_{\mathrm{d}}\right) f_{\mathrm{p}} f_{\mathrm{f}} Q_{\mathrm{f}}+f_{\mathrm{s}} C_{\mathrm{s}} Q_{\mathrm{s}}+C_{\mathrm{w}} f_{\mathrm{w}} Q_{\mathrm{w}}\right]
$$

where $C_{\mathrm{i}}$ is the concentration of DU in deer tissue, $F_{\mathrm{a}}$ is the transfer coefficient of ingested DU to deer tissue, $C_{\mathrm{a}}$ is the DU concentration that passes into plant roots from soil and is incorporated into the plant tissue, $C_{\mathrm{d}}$ is the DU concentration deposited on the surface of plants eaten by deer, $f_{\mathrm{p}}$ is the fraction of time deer spend in the contaminated area, $f_{\mathrm{f}}$ is the fraction of the fodder supply that is contaminated, $Q_{\mathrm{f}}$ is the consumption rate of vegetation by deer, $f_{\mathrm{s}}$ is the fraction of the ingested soil that is contaminated with $\mathrm{DU}, C_{\mathrm{s}}$ is the DU concentration in the soil, $Q_{\mathrm{s}}$ is the soil ingestion rate by deer, $C_{\mathrm{w}}$ is the DU concentration in water drunk by deer, $f_{\mathrm{w}}$ is the fraction of the water that is contaminated, and $Q_{\mathrm{w}}$ is the consumption rate of water by deer. The term $f_{\mathrm{s}} C_{\mathrm{s}} Q_{\mathrm{s}}$ describes the soil ingestion component of $\mathrm{C}_{\mathrm{i}}$, the $C_{\mathrm{w}} f_{\mathrm{w}} Q_{\mathrm{w}}$ term describes the water ingestion component of $C_{\mathrm{i}}$, and the remaining term describes the contribution of DU from plants. The DU concentration absorbed through plant roots and incorporated into plant tissue, $C_{\mathrm{a}}$, is calculated by

$$
C_{a}=\frac{B_{1} d_{1}}{P}\left(\frac{1-\exp \left(-\lambda_{1} t_{\mathrm{b}}\right)}{\lambda_{1}}\right),
$$

where $d_{1}$ is the DU inventory available to plant roots, $B_{1}$ is the concentration ratio for soil to plants (NCRP 1984a), $P$ is the soil bulk density divided by the depth of the rooting layer, $\lambda_{1}$ is the removal rate or leaching rate of DU from the soil, and $t_{\mathrm{b}}$ is the time the DU accumulates in the contaminated area.

The inventory of DU in the soil, $d_{1}$ in Equation 3-17, is assumed to be $100 \%$ available to plant roots. The actual chemical composition of DU in the field includes DU metal, schoepite (which is the only corrosion product identified), and "soluble" $\mathrm{UO}_{2}{ }^{2+}$ or $\mathrm{U}^{4+}$. "Soluble" $\mathrm{U}$ can be truly in solution, adsorbed onto clay minerals, or complexed with naturally occurring organic and inorganic substances. The concentration of DU available for absorption by plant roots is that fraction that is soluble as $\mathrm{UO}_{2}^{2+}$ or $\mathrm{U}^{4+}$. The amount of DU in solution in these forms is controlled by the rate of dissolution of the solid phases, either DU or schoepite, and the solution concentrations can range from less than $1 \mathrm{pCi} / \mathrm{L}$ to about 4000 $\mathrm{pCi} / \mathrm{L}$. Most of the DU in the soil is present as one of the solid phases because of the low solubility of either solid phase. The actual contribution of $d_{1}$ to the total $U$ in plants is small compared with the amount ingested by animals eating vegetation coated with resuspended soil.

The rate of removal of DU from soil in Equation 3-17 is defined as

$$
\lambda_{1}=\lambda_{t}+\lambda_{L}+\lambda_{H},
$$

where $\lambda_{t}$ is the loss of DU due to radioactive decay, $\lambda_{L}$ is the loss of DU due to leaching through the soil, and $\lambda_{\mathrm{HI}}$ is loss of DU due to harvest and removal of vegetation from the food supply. Since DU has an extremely long half-life $\left({ }^{235} \mathrm{U}=7.1 \times 10^{8}\right.$ years, ${ }^{234} \mathrm{U}=2.5 \times 10^{5}$ years, and ${ }^{238} \mathrm{U}=4.5 \times 10^{9}$ years $)$, the amount of DU lost through radioactive decay per day is small and does not contribute significantly to $\lambda_{1}$. 
There is no harvest or weed removal reported at APG except for vegetation consumed by deer and other animals, so the loss rate due to harvest is 0 . Thus, $\lambda_{1}$ depends only on leaching of DU through the soil. A simple leaching model was used (NCRP 1984a) and is described as

$$
\lambda_{1}=\lambda_{\mathrm{L}}=\frac{V_{\mathrm{w}}}{D_{\mathrm{s}}\left[1+\left(\frac{\rho}{\theta} K_{\mathrm{d}}\right)\right]},
$$

where $V_{\mathrm{w}}$ is the velocity of water percolating downward through the soil, $D_{\mathrm{s}}$ is the depth of the root zone, $\rho$ is the bulk density of the soil, $\theta$ is the volumetric water content of the soil, and $K_{\mathrm{d}}$ is the distribution coefficient or the ratio at equilibrium of DU adsorbed on the soil particles to the DU concentration in the soil water.

The concentration on the surface of foliage, $C_{\mathrm{d}}$ in Equation 3-16, was calculated using

$$
C_{d}=\frac{f_{r} T_{v} d_{2}}{Y_{v}}\left(\frac{1-\exp +\lambda_{E} t_{c}}{\lambda_{E}}\right),
$$

where $f_{\mathrm{r}}$ is the fraction of material intercepted by the plant surface, $T_{\mathrm{v}}$ is the translocation factor to the edible portions of the plant, $d_{2}$ is the deposition rate and is defined as the amount of DU deposited from the air onto plant surfaces, $Y_{v}$ is the standing plant biomass at the end of the growing season, $\lambda_{\mathrm{E}}$ is the removal constant of DU from the plant surface, and $t_{\mathrm{e}}$ is the time the plant has been exposed to DU at the rate specified by $d_{2}$. The deposition rate from air is the amount of DU that is deposited after a penetrator impacts contaminated soil and lifts the soil into the air. Ambient winds also result in resuspended DU but less frequently than resuspension from penetrator impacts. The rate and amount of deposition depend on the soil concentration of DU in the area where the penetrators impact and on the amount of DU on particles small enough to be transported in the air. From measurements and field observations at APG, only a fraction of the total inventory is available for transport by resuspension because some of the fragments are deposited in areas of infrequent impact, some fragments are too large to be deposited on plant surfaces, and some fragments are buried too deeply for released particles to be resuspended .

We used data from YPG (Price 1991) and APG (Larry Davis, personal communication) to estimate probable locations within the impact areas that would result in the largest surface deposition rates and therefore the greatest DU deposition on plant surfaces. The location at YPG that is most likely to result in large values of $d_{2}$ is about $4000 \mathrm{~m}$ downrange from GP17A (Appendix A of Price 1991). Assuming that the impact area of GP17A is as indicated, the area of greatest concentration is conservatively estimated at $5 \%$ of the total impact area and extends about $500 \mathrm{~m}$ downrange of and $50 \mathrm{~m}$ perpendicular to the firing line. A similar estimate of the size of the affected area was made from soil transect data and surface survey information. The total DU concentration in the affected area is estimated to be no greater than about $10 \%$ of the total inventory deposited on the entire impact range using Price's data (Price 1991) and soil data from APG. Using this information, the deposition rate was calculated by taking the fraction of the total inventory that is available for resuspension and dividing it by the area of potential deposition. DU recovery from APG showed that there are two areas that could be most susceptible to conditions favoring resuspension of small particles, and both were included in the model. 
The value of the removal constant, $\lambda_{\mathrm{E}}$, is calculated using

$$
\lambda_{\mathrm{E}}=\lambda_{\mathrm{t}}+\frac{\ln 2}{t_{\mathrm{w}}},
$$

where $\lambda_{\mathrm{t}}$ is the radioactive half-life and $t_{\mathrm{w}}$ is the time required for one-half of the deposited DU to be lost from the surface of the plant. Since $\lambda_{1}$ (per day) is small with respect to the second term of Equation 3-21, $\lambda_{\mathrm{E}}$ is effectively an inverse function of only $t_{\mathrm{w}}$. The values, ranges, and statistical distributions of the variables listed in Equations 3-16-3-21 are tabulated in Appendix B.

\subsubsection{Sensitivity and Uncertainty Analyses}

Estimating the DU concentration in deer meat consumed by humans $\left(C_{i}\right.$ in Equation 3-16) also requires estimating the uncertainty in the statistical distribution of $C_{i}$. The calculations discussed above were used in a Monte Carlo simulation to show the effects of variation in the input parameters on the estimated DU concentration in deer tissue. LHS was not used in evaluating the terrestrial model because the model is mathematically simple and can be efficiently tested using Monte Carlo methods. The Monte Carlo simulation is an iterative approach for determining the uncertainty in the estimate of $C_{i}$ and works similarly to the LHS method discussed for the aquatic model. The main difference from the LHS method is that in the Monte Carlo method the distribution of input variables is not divided into a specific number of segments with equal probabilities of being selected. In simulations with few iterations, the Monte Carlo method tends to underrepresent input values on the tails of distributions. In simulations with large numbers of iterations, however, the difficulty with underrepresentation is decreased. The probability distribution of the input parameters in Monte Carlo simulations is analogous to its role in LHS.

In a Monte Carlo simulation, one value of each parameter in the model (Equation 3-16, including the contributions from Equations 3-17-3-21) is chosen at random from the range and within the probability distribution of that parameter. The chosen values are then used to calculate a single-point estimate of $C_{i}$ in Equation 3-16. After the first iteration, new values of each parameter are selected at random and a new value of $C_{i}$ is calculated. The simulation continues for any number of iterations, thereby generating a probability distribution that indicates the variation in the estimates of $C_{i}$. Estimates of the uncertainty in $C_{i}$ are most accurate when a large number of iterations is used.

We used the values and distributions listed in Appendix B (Tables B-21 and B-22) to estimate the uncertainty in the predicted values of $C_{i}$, the concentration of DU in deer tissue (Table 3-4). Distributions for input parameters were constructed when sufficient data were available. Data from field sampling were used extensively to construct input parameter distributions. Some parameters, such as soil bulk density or downward percolation velocity in Equation 3-19, were insensitive with regard to their effect on the predicted DU concentration in deer tissue and were estimated from the literature or other sources (NCRP 1984a; USDA 1975). Uniform distributions were used for insensitive parameters and were based on the range of values reported. 
Table 3-4. Various statistics from the uncertainty analysis of the steady-state model for predicted values of $C_{i}$.

\begin{tabular}{|l|c|}
\hline \multicolumn{1}{|c|}{ Statistic } & Value \\
\hline Number of Trials & 10,000 \\
\hline Mean & $2.2 \times 10^{-4} \mathrm{pCi} / \mathrm{g}$ \\
\hline Median & $1.7 \times 10^{-4} \mathrm{pCi} / \mathrm{g}$ \\
\hline Mode & $1.4 \times 10^{-4} \mathrm{pCi} / \mathrm{g}$ \\
\hline $\begin{array}{l}\text { Standard Deviation } \\
\text { Range } \\
\text { Minimum } \\
\text { Maximum }\end{array}$ & $\begin{array}{c}2.3 \times 10^{-4} \mathrm{pCi} / \mathrm{g} \\
0.0 \times 10^{-3} \mathrm{pCi} / \mathrm{g}\end{array}$ \\
\hline Skewness & 6.38 \\
\hline
\end{tabular}

\subsubsection{Results and Discussion}

Equations 3-16-3-21 were used in Monte Carlo simulations to evaluate the parameters that were most influenced by variations in input values. Monte Carlo simulation was used to evaluate which parameters were the most sensitive. To estimate the sensitivity of the model, the parameters were varied at random within their ranges, just as they were in the uncertainty analysis. However, only one parameter per simulation was varied, and the remaining parameters were held at fixed values. Ten thousand iterations were run, and then the probability distribution was constructed and statistics were determined for each family of $C_{i}$. The standard deviation of the estimated $C_{i}$ was one measure of the variation caused by each parameter. Parameters that resulted in the largest variation in $C_{i}$ values were considered sensitive parameters.

Each equation describes a particular part of the model and, therefore, each has a set of sensitive parameters. The contribution of some of the sensitive parameters overshadows the effects of others when the whole model is considered, thereby rendering insensitive some parameters that were at one level considered sensitive. One example is the calculation of $\lambda_{L}$, the amount of DU lost through soil leaching, in Equation 3-18. The velocity with which water flows vertically through the soil profile is not known from field measurements. One report (U.S. Army 1990b) states that the velocity of groundwater flow is "several inches per day" and leaves significant uncertainty in the $V_{w}$ parameter. While the value of $\lambda_{L}$ depends on $V_{\mathrm{w}}$ in Equation 3-19, variations of $V_{\mathrm{w}}$ had no significant effect on the calculated value of $C_{i}$ (Equation 3-16) because other variables were more influential. Distributions for all variables listed in Equations 3-16 through 3-21 were constructed initially, then parameters such as $V_{\mathrm{w}}$ were eliminated to simplify the analyses.

After the parameters that contributed less than $1 \%$ to $C_{i}$ were eliminated, the sensitivity analysis showed that soil concentration ( $C_{s}$, Equation 3-16), dust mass in the air (imbedded in the calculation of $d_{2}$, Equation 3-20), and the weathering half-time ( $t_{\mathrm{w}}$, Equation 3-21) explained about $98 \%$ of the variance in 
the predicted values of $C_{i}$. Table 3-5 shows the six most influential parameters in the model in terms of the percent to which they contribute to the variance in $C_{i}$.

Table 3-4 shows that the distribution of values of $C_{i}$ is positively skewed and indicates that the probability of a value less than the mean is much greater than the probability of a value greater than the mean. The median value of $C_{i}$ in Table 3-4 shows that there are equal numbers of occurrences above and below $1.7 \times 10^{-4} \mathrm{pCi} / \mathrm{g}$, and the mode shows that the most probable estimate of $C_{i}$ is about $1.4 \times 10^{-4}$ $\mathrm{pCi} / \mathrm{g}$. The standard deviation of the mean is slightly larger than the mean (about $2.3 \times 10^{4} \mathrm{pCi} / \mathrm{g}$ ) and suggests that an estimated value of $C_{i}$ could range from 0 to about $5.0 \times 10^{-4} \mathrm{pCi} / \mathrm{g}$.

Table 3-5. Contributions of the six parameters that most affect $C_{\mathrm{i}}$, the concentration of DU in deer tissue. The results of 10,000 iterations were used to generate the distribution of predicted values.

\begin{tabular}{|l|c|}
\hline \multicolumn{1}{|c|}{ Parameter } & \% of Total Variance \\
\hline DU Concentration in Soil, $C_{\mathrm{s}}$ & 41.4 \\
Airborne Dust $\left(d_{2}\right.$ In Equation 3-20) & 34.8 \\
Weathering Time ( $t_{\mathrm{w}}$, Equation 3-21) & 22.5 \\
Feed Intake Rate (Equation 3-16) & 0.8 \\
Soil Ingestion Rate (Equation 3-16) & 0.5 \\
Water Intake Rate (Equation 3-16) & 0.05 \\
\hline
\end{tabular}

Of the three components described in Equation 3-16, soil ingestion contributes the most to $C_{i \text {.. }}$ The magnitude of the soil ingestion term depends primarily on the DU concentration in the soil, and the soil ingestion rate plays a secondary role. Table 3-5 illustrates the dominance of $C_{\mathrm{s}}$ in the soil ingestion term. Two of the parameters that determine the amount of DU on the surface of plants are the mass of dust resuspended after impacts and the weathering time or the rate at which DU on surfaces is washed off to the soil. These two parameters are the next most important ones after soil concentration and account for about $57 \%$ of the variance.

While the contribution to $C_{i}$ from DU taken into plants through roots from soil is small, there are other important factors. Equation 3-16 shows the effect of DU internally deposited in plants through plant roots. The small magnitude of the bioconcentration factor indicates that little DU is taken into plants through their roots. The amount of DU absorbed through plant roots would increase with higher soil concentrations, lower leaching rates, an increased percentage of DU in finely divided particles, and moreextensive root systems within the contaminated soils. Relatively high concentrations of $U$ and other metals have been found in plants, but high plant concentrations occur in areas of much greater $U$ availability than the impact area (Ibrahim and Whicker 1988).

In this model, the DU deposited on plant surfaces contributes more to $C_{i}$ than the DU absorbed through the roots. Equation 3-20 shows the parameters that are used to calculate the amount of DU 
deposited on the surface of plants. The most influential parameters are the size of the area containing the available DU, discussed previously, and the biomass that is available as a depositional surface for DU and, therefore, as food for the deer. The interception fraction of the plants and the translocation factor are important but less so than the biomass and area of contamination parameters. The dependence of $C_{\mathrm{d}}$ on the area available for resuspension, the biomass, the translocation factor, and the interception fraction shows the importance of the density of the plants used for food and the amount of area covered by the plants. Interactions of different factors can significantly alter the model output if a large area for resuspension is used in the modeling or if a large biomass is associated with an area.

\subsection{APG Field Sample Collection}

\subsubsection{Introduction}

Concentrations of DU in the environment are likely to reflect the total amount of uranium introduced into the impact areas. Spatial patterns of DU concentrations also approximate the distribution of penetrator fragments in soils and sediment. The chemical instability of $U$ metal in the ambient environment results in oxidation of the $U$ fragments and the transport of soluble $U$ constituents or small particles. Potential contamination of a large area such as the Main Front Firing Range is possible because of the amount of DU munitions tested at APG. Due to the nature of munitions testing, spatial and temporal concentrations in soils and sediments are expected to vary considerably.

It is unclear how much uranium would be taken up by living organisms, even though measurable amounts of DU are expected in sediments and water. Uranium has been shown to accumulate in living tissue with concentrations decreasing with successive trophic levels of the food chain (Kovalsky et al. 1967; Thompson et al. 1972; Blaylock and Witherspoon 1976; Mahon 1982). Consequently, we would expect to find the highest concentrations in phytoplankton and the lowest levels in carnivorous fish (e.g., sunfish [Centrarchidae] and white perch [Morone americanus]).

The total amount of uranium accumulated in aquatic ecosystems appears to be site-specific (Mahon 1982). Uranium uptake in the environment depends on the amount and chemical forms of uranium present in the environment, their spatial distribution, the type of biota present in the area, and individual physiological capabilities to bioaccumulate uranium, as well as abiotic factors such as the physicochemical characteristics of DU in water and the amount of DU that can bind to local soils and sediments (Osburn 1974 [in Mahon 1982]; Brenchley et al. 1977; Mahon 1982). To obtain information on uranium concentrations and their spatial distribution at APG, field samples were collected from many of the trophic compartments. Biota containing DU could indicate the bioaccumulation of corrosion products from penetrators that might represent toxicological or radiological hazards or both. 


\subsubsection{Materials and Methods}

Field sampling locations 5-8 were selected to maximize the chance of detecting DU in the environment; thus, nonrandom site selection was used (Figure 3-5). Selection of sampling sites was based on estimates of where the highest DU concentrations might be found using information from penetrator recovery efforts and on where tidal flows could potentially transport corrosion products off-site. Penetrators were typically not visible due to heavy vegetation and turbid water.

Field collections were made in APG impact areas during 1992 (25-26 July and 10-11 October 1992). Sampling time at APG was limited by firing schedules and the availability of explosive-ordnance demolition personnel. A total of 11 sites were selected, and 394 samples were collected during two sampling trips. Biotic and abiotic samples were collected from as many different environmental compartments as possible at each sampling location. Because of the diversity of taxa, the number of samples, and the nonrandom locations, samples collected were likely to contain DU, if it were present in the sampled media.

Field sampling techniques employed during the July and October trips were identical. A portion of the July field samples were analyzed prior to the October trip to identify environmental compartments, such as sediment, that contained DU. Based on the early analyses, the compartments containing DU were collected preferentially during the October trip. Additional trips were made to collect soil samples and water data from selected surface locations and wells installed by the U.S. Army Corps of Engineers (U.S. Army 1990b).

Concentrations of different isotopes of $U$ were used to evaluate the source of $U$ in the samples. Natural $U$ has a nominal mass ratio of ${ }^{235} U{ }^{238} U$ of 0.0072 , whereas the DU used in U.S. Army penetrators has a nominal mass ratio of ${ }^{235} \mathrm{U} /{ }^{238} \mathrm{U}$ of 0.0020 . Samples with mass ratios significantly less than 0.0072 are considered to contain DU, and samples with mass ratios near 0.0020 are considered to come from DU penetrator corrosion. In our analyses, samples with ratios less than 0.0065 (the lower $95 \%$ confidence interval of the mean of 0.0072 ) were categorized as potentially containing DU from penetrators, and samples with ratios from 0.0010 to 0.0032 were categorized as containing DU from penetrators. Samples with ratios between 0.0032 and 0.0065 could contain both $D U$ and natural $U$, or there could be an error in the reported concentrations of ${ }^{235} \mathrm{U},{ }^{238} \mathrm{U}$, or both. 


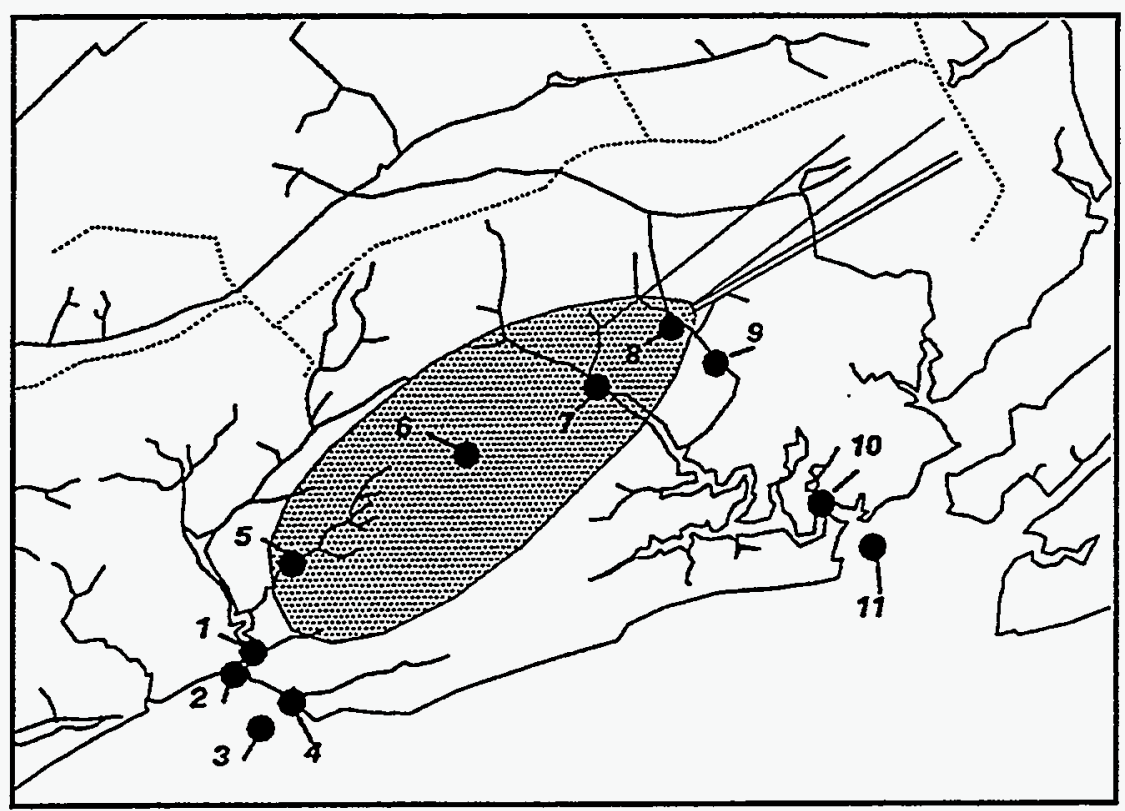

\begin{tabular}{|c|c|}
\hline $\begin{array}{c}\text { Map Identification } \\
\text { Number }\end{array}$ & Site Name \\
\hline 1 & Delph Creek \#1 \\
\hline 2 & Delph Creek \#2 \\
\hline 3 & Old Woman's Gut \#2 \\
\hline 4 & Old Woman's Gut \#1 \\
\hline 5 & Upper Delph Creek \\
\hline 6 & DU Road \\
\hline 7 & Upper Mosquito Creek \\
\hline 8 & B3 Catch Box \\
\hline 9 & B3 Creek \\
\hline 10 & Mosquito Creek \#1 \\
\hline 11 & Mosquito Creek \#2 \\
\hline
\end{tabular}

Figure 3-5. Sampling locations at APG in July 1992 and October 1992. 


\subsubsection{Analytical Methods}

\subsubsection{Neutron Activation}

Several instrumental analytical methods were used for analysis of $U$ and its isotopes. Neutron activation analysis (NAA) was considered an important tool and was available at Los Alamos for use at the beginning of our study. The effectiveness of NAA for environmental samples has been shown previously (Gladney et al. 1983, 1980, 1978, 1976). Analysis of small samples and the need for minimal preparation make NAA ideal for determining the $U$ concentration in a large number of samples.

Samples were prepared for NAA by weighing the fresh samples ("wet weight"), drying them at $120^{\circ} \mathrm{C}$ for at least 8 hours, weighing the samples again after the drying period ("oven-dry weight"), packing the dried sample into a tared plastic NAA capsule, or rabbit, and then measuring the net wet of the sample in the rabbit by the difference. Weight data were recorded for later reference. Samples were submitted to the Omega West Reactor Facility at Los Alamos for analysis. Data on U isotopes and concentrations of several other elements were transferred electronically to EES-15.

Sediment and tissue samples were also prepared for analysis by inductively coupled plasma-mass spectroscopy, also at Los Alamos. Initial "wet" weights of all samples were recorded, then samples were dried at $120^{\circ} \mathrm{C}$. Dry weights were recorded, and sediment samples were packaged in storage containers. Biological samples were then ashed in a muffle furnace programmed to hold them at $250^{\circ} \mathrm{C}$ for 3 hours, $350^{\circ} \mathrm{C}$ for 3 hours, and finally $450^{\circ} \mathrm{C}$ for four hours. Ash weights were recorded, and samples were packaged for later analysis and storage.

Samples prepared for NAA were delivered to the Omega West Reactor Facility at Los Alamos. Samples were queued for injection into the reactor. After injection and irradiation for approximately 30 seconds, the samples were remotely transferred to the counting facility. For delayed neutron activation (Gladney et al. 1978, 1980; Amiel 1962; Coleman and Pierce 1967), samples were irradiated by a flux of neutrons; after a 25- to 35-s delay, the samples were counted in a boron trifluoride neutron detector. Gamma radiation emitted from ${ }^{239} U$ (an activation product) and ${ }^{235} U$ were quantified and used to determine the amount of ${ }^{238} U$ and ${ }^{235} U$ in each sample. Actual $U$ concentrations in each sample were determined from the total $U$ in the sample divided by the mass of the dried sample.

Delayed neutron activation provides isotopic ratio data that allows differentiation of natural $U$ and DU. Samples collected in the field were expected to contain either background concentrations of natural $\mathrm{U}$ or DU from the corrosion of spent penetrators. Detection limits ranged from 0.22 to $5 \mu \mathrm{g}$ for ${ }^{235} \mathrm{U}$ and 0.07 to $4 \mu \mathrm{g}$ for ${ }^{238} \mathrm{U}$. These detection limits correspond to concentrations of approximately 0.1 to $2.5 \mu \mathrm{g} / \mathrm{g}$ for ${ }^{235} \mathrm{U}$ and 0.04 to $2 \mu \mathrm{g} / \mathrm{g}$ for ${ }^{238} \mathrm{U}$. The detection limits varied because of self-absorption of the gamma signal from the sample by the sample matrix. Quality assurance samples were included and consisted of traceable reference standards from NIST, as well as environmental samples from previous APG sampling efforts (Ebinger et al. 1990). Standards and previously collected environmental samples were submitted without the analyst's knowledge. The results of the analyses of the NIST standards were within acceptable limits of the reported quantities, as were the results of analyses of the previously collected samples. 
NAA data were reported as total $U$ based on the activity of ${ }^{235} U$ and total $U$ based on the activity of ${ }^{239} \mathrm{U}$ in a sample. ${ }^{239} \mathrm{U}$ results from the activation of ${ }^{238} \mathrm{U}$ during irradiation. The ratio of total $U$ from ${ }^{235} U$ to total $U$ from ${ }^{239} U$ provides information on the source of the $U$. Ratios less than 0.8 indicate that the sample contains a significant amount of DU, whereas ratios between 0.9 and 1.1 indicate that the U was derived from natural sources. Absolute masses of ${ }^{235} \mathrm{U}$ and ${ }^{238} \mathrm{U}$ were not measured in these analyses.

The Omega West Reactor Facility was closed for extensive repairs after an initial set of samples were analyzed and the data reported. In early 1993 ICP-MS was selected as the main analytical tool for the duration of this project because the Omega West Reactor Facility would not be available for further analyses.

\subsubsection{Ion Chromatography}

Ion chromatography (IC) was also used for the analysis of total U in samples. Unlike NAA, IC could not be used for isotope identification and quantification, so data from IC pertain only to the total amount of $U$ in a sample. IC involves separating the ionic constituents of a solution via a chromatographic column, complexing the U with a UV-sensitive reagent, and then measuring the absorption of light by the $\mathrm{U}$-complex. The amount of $\mathrm{U}$ in the solution is related to the quantity of light absorbed by the sample.

Samples were extracted using Environmental Protection Agency (EPA) methods (EPA 1987, 1991). Extracts were obtained by acid digestion followed by peroxide oxidation and dilution to approximately $\mathrm{pH} 2$. The resulting solutions were diluted as needed and analyzed. We used a Dionex 4500i Ion Chromatograph equipped with a variable-wavelength detector, HPIC CS2 column, Spectra Physics

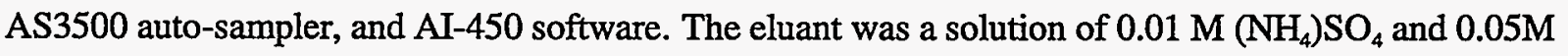
$\mathrm{H}_{2} \mathrm{SO}_{4}$. The complexant was a solution of $2 \times 10^{4} \mathrm{M} 4$-(2-pyridylazo) resorcinol (PAR), $3 \mathrm{M} \mathrm{NH}_{4} \mathrm{OH}$, and $1 \mathrm{M} \mathrm{CH}_{3} \mathrm{COOH}$. The variable-wavelength detector was optimized near $520 \mathrm{~nm}$ to detect the $\mathrm{UO}_{2}{ }^{2+}-\mathrm{PAR}$ complex. Extracted samples were diluted and sealed in clean sample vials fitted with teflon septa and then placed into the auto-sampler for analysis. After injection, samples were mixed with eluant and PAR reagent and admitted to the separation column. Concentration data were collected and analyzed via the AI-450 software.

Calibration standards were prepared from NIST-traceable $\mathrm{UO}_{2}\left(\mathrm{NO}_{3}\right)_{2}$ solution. Standards from 0 to 20 $\mathrm{mg}-\mathrm{U} / \mathrm{L}$ were prepared and run in the batch with the samples. At least three sets of standards were run with each batch of samples. Calibration standards were run before and after all samples and at least once during the run to ensure that the instrument was calibrated. Multiple calibrations within each batch of samples allowed evaluation of the quality of the data at all times during the analyses. The IC system functioned acceptably throughout the analysis period.

Method detection limits from water matrices were in the low micrograms per liter range. The extraction method, however, caused extreme interference with complex formation and/or the elution of $U$ peaks from the column. Because of the interference, the $\mathrm{pH}$ of the extracts was adjusted to between $\mathrm{pH}$ 2.5 and 3.5 before injection. The increased dilution that resulted from the $\mathrm{pH}$ adjustments made the detection limit $1 \mathrm{mg} / \mathrm{L}$ at best. Extracting $U$ from samples such as biological tissue or plant material, 
therefore, was of little value. Conversely, U extracted from soils or sediments that were highly contaminated ( $>100 \mathrm{mg}-\mathrm{U} / \mathrm{kg}$, in some cases) was easily detected.

Reproducibility of calibration curves was not always achievable. Significant differences in the slopes of calibration curvès were observed when calibrations from different batches were compared. However, no significant differences were observed between the three or more calibration curves from the same batch of samples. All calibration data were retained with the appropriate sample data in order to provide a means for checking the reported concentrations if needed. Sample analysis time was approximately 17 minutes per sample. The IC system was in continuous use during most of the analytical period.

\subsubsection{Inductively Coupled Plasma-Mass Spectroscopy}

Inductively coupled plasma (ICP) used in conjunction with mass spectroscopy (ICP-MS) has also been effectively used to determine total $U$ and isotopic $U$ concentrations in environmental samples (Gladney et al. 1989; Ebinger et al. 1990). The extraction procedure is similar to that used for IC analyses without the need for the additional $\mathrm{pH}$ adjustments required for IC. The mass spectroscopic capability allows quantification of the mass concentrations of ${ }^{238} \mathrm{U}$ and ${ }^{235} \mathrm{U}$ if both are above the detection limits.

The analytical options were to use $\alpha$-spectroscopy or ICP-MS. ICP-MS was significantly less expensive per sample than $\alpha$-spectroscopy and has similar detection limits. Samples intended for ICP-MS analyses were shipped by overnight courier to Core Laboratories for analysis. ${ }^{1}$ Core Laboratories personnel completed a Receipt of Acknowledgment form for each sample and returned completed forms to Los Alamos. U or DU in each sample was extracted using EPA Method 3050 (EPA 1987). The method involves extraction of metals with hot, concentrated nitric and hydrochloric acids, then oxidation of organics with hydrogen peroxide. The extracts were separated from the remaining solids, filtered, and diluted for ICP-MS analysis. ICP-MS analyses were conducted using EPA Method 200.2 (EPA 1991).

Quality assurance and quality control (QA/QC) measures were followed as prescribed in EPA Method 6020 (EPA 1991). A series of QA/QC blanks, standards, duplicates, and spikes were analyzed with each set of samples. Instrumental and method blanks demonstrated that there was no uranium contamination in the samples as a result of sample extraction. ${ }^{238} \mathrm{U}$ samples were run after calibration with certified laboratory standards of 0.5 to $200 \mu \mathrm{g} / \mathrm{L}$. Each batch of samples and standards showed the expected and actual values of the standards for comparison and to demonstrate that the instrument was calibrated. ${ }^{235} \mathrm{U}$ samples were run in the same manner as the ${ }^{238} \mathrm{U}$ samples, except that the laboratory standard was derived from weathered DU collected from YPG. The DU had an isotopic ratio $\left({ }^{235} U / /^{238} U\right)$ of $0.0021 \pm 0.0004$ that was determined independently. The calibration range for ${ }^{235} \mathrm{U}$ was 1 to $200 \mu \mathrm{g}-\mathrm{U} / \mathrm{L}$.

Matrix spikes were samples of $1.2,1.5$, and $46 \mu \mathrm{g}-\mathrm{U} / \mathrm{L}$ to which spikes of $50 \mu \mathrm{g}-\mathrm{U} / \mathrm{L}$ were added. These spiked samples provided a check on the recovery of analyte during the analyses. Duplicates of the $1.2-, 1.5-$, and $46-\mu \mathrm{g} / \mathrm{L}$ samples were run and compared to ensure proper instrument function.

Concentrations determined through ICP-MS (in $\mu \mathrm{g}-\mathrm{U} / \mathrm{L}$ ) were converted to $\mu \mathrm{g}-\mathrm{U} / \mathrm{kg}$ by adjusting the

\footnotetext{
${ }^{1}$ Use of Core Laboratories does not constitute endorsement by Los Alamos National Laboratory.
} 
reported ICP-MS concentration for the dilution factors and the weight of the sample. The resulting concentrations for ashed or oven-dried samples were reported to Los Alamos by Core Laboratories.

\subsubsection{Field Instruments}

Several field instruments were used during sample collection trips. Dissolved oxygen was measured from surface and groundwaters using a YSI Model 73 dissolved oxygen meter and a portable Corning dissolved oxygen meter. ${ }^{1}$ Both the YSI and the Corning meters were calibrated and checked against a known standard before each use in the field. A portable oxidation-reduction potential (ORP) pen indicator was used to estimate the redox potential of water samples and to obtain additional estimates of dissolved oxygen concentrations. Several portable $\mathrm{pH}$ meters were used for $\mathrm{pH}$ measurements in water. The appropriate commercially available standards were used to calibrate all meters before each use and to verify calibration during use.

\subsubsection{Water Chemistry and Stream Flow Measurements}

Volume flow rates of Mosquito Creek were measured on two occasions, and two sites were selected. The first site was near the headwaters of Mosquito Creek, approximately $100 \mathrm{~m}$ downrange from the Trench Warfare Catch Box. The second site was the mouth of Mosquito Creek, approximately $1.5 \mathrm{~km}$ downstream from the first site. The area of the channel at each site was estimated by measuring the depth of a section perpendicular to the flow of the stream.

Water samples were collected at sampling locations during both trips. Visibility (turbidity) and water depth were measured in the field to the nearest $0.1 \mathrm{~m}$ using a Seichi disk. Dissolved oxygen and temperature were measured with a YSI Model 57 oxygen meter. Salinity and conductivity were measured with a YSI Model 33 conductivity meter, and $\mathrm{pH}$ was measured with a Jenco Model $60009 \mathrm{pH}$ meter. All instruments were calibrated prior to use according to the manufacturer's directions, and probes were rinsed with $10 \%$ nitric acid and ultrapure water between uses to prevent cross-contamination.

Approximately $3 \mathrm{~L}$ of unfiltered water were collected at all sites and stored in clean (certified) plastic cubitainers. Water was acidified with analytical grade nitric acid (Mallinkrodt Specialty Chemical Co.) to pH 3 and frozen for shipment to Los Alamos. Freezing and acidification are preservation techniques that minimize surface adsorption of uranium to container surfaces.

Well samples were collected during the October trip and on additional trips throughout 1992 and 1993. Temperature, dissolved oxygen, $\mathrm{pH}$, alkalinity, and oxidation-reduction potential were measured in the field at each of the wells before sampling. The oxidation-reduction potential was measured with an ORP pen instrument; all other measurements were made with the same instruments as those used for water samples collected from the sample locations shown in Figure 3-6. Any samples collected for U analysis were preserved by acidification and freezing. Chelex $100 \mathrm{Resin}$ (BioRad) was used to concentrate $U$ isotopes from water samples. The resin was washed to remove impurities, then converted to the ammonium form to exchange cations in the water for the ammonium ions on the resin (Greenberg

\footnotetext{
${ }^{1}$ Use of specific trade names does not constitute endorsement by Los Alamos National Laboratory.
} 
and Kingston 1982; Greenberg and Kingston 1983; Kingston et al. 1978; Kingston and Greenberg 1984). After a sample was eluted through the resin column, the $U$ was removed by acid leaching (Kingston and Greenberg 1984) if the samples were intended for IC analysis or the resin was dried for NAA analysis.

\subsubsection{Sampling Procedures}

Hand-held seines and kick nets were used to collect fish and benthic macroinvertebrates in shallow areas. Taxa from several different aquatic feeding guilds were collected, including suspension feeders (e.g., creek chub [Semotilus atromaculatus]), benthic detrivores (e.g., blue crabs [Callinectes sapidus], tadpoles [Rana sp.], and grass shrimp [Paleomonetes sp.]), and carnivores (mummichog [Fundulus heteroclitus], dragonfly naiads [Gomphus sp.], giant water bugs [Belastoma sp.], American eel [Anguilla rostrata], sunfish [Centrarchidae], and pickerel [Esox americanus]). Sediment was also collected from each sampling location using a stainless steel scoop. Sediment samples were frozen before they were shipped to Los Alamos for further analysis.

In deeper water, samples were collected using an otter trawl at the mouths of Mosquito Creek and Delph Creek. A plankton tow net with $80-\mu \mathrm{m}$ mesh was used to collect phytoplankton, zooplankton, and suspended particulate matter. Sediment and benthic invertebrates were collected with a Ponar sampler. Benthic invertebrates were retrieved from sediment by washing the sediment through a 1.0 -mm sieve with de-ionized (DI) water and removing organisms from the remaining litter. Sorted invertebrates were rinsed with DI water, sealed in plastic bags, and frozen. Aquatic macrophytes were collected by hand, rinsed with DI water, and frozen in plastic bags.

All samples were sorted to the lowest possible taxa in the field and frozen for shipment to Los Alamos for total and isotopic uranium analyses. Kidney and bone tissues were dissected from larger organisms since uranium tends to preferentially accumulate in these tissues. Individual organisms and individual organs were used for uranium analyses whenever practical, but restricted numbers of samples and small biomass forced pooling of some samples. All dissection equipment was rinsed with $10 \%$ nitric acid and ultrapure water to prevent cross-contamination.

Deer were harvested by way of special permits from the Maryland Department of Game and Fish. Deer were dissected on top of a plastic cloth spread on the soil surface to minimize contamination from DU-containing soil. Samples of liver, kidney, muscle, and bone tissues were collected using tools cleaned with a $10 \%$ nitric acid solution and rinsed with DI water. Tools were cleaned before and after each tissue sample was taken. The plastic ground cloths were disposed of after a single use.

Field sampling was often serendipitous. The July 1992 collection included a moribund great blue heron (Ardea herodias). Since herons feed predominately on fish and may accumulate DU from the environment as a consequence, tissue samples from the heron were analyzed for uranium content. Tissue samples included vertebral bone, pectoral muscle, kidney, liver, and portions of the gastrointestinal tract. 


\subsubsection{Results}

\subsubsection{Groundwater and Surface Water}

Surface water quality parameters at APG sampling sites are summarized in Tables 3-6 and 3-7. Generally, the October data show lower temperatures and corresponding higher dissolved oxygen concentrations, higher visibility (that is, the depth at which the Seiche disk is visible), and higher salinity and conductivity. Data from groundwater wells (Table 3-8) show significantly cooler water temperatures, lower dissolved oxygen, lower oxidation-reduction potentials, relatively low alkalinity, and stable $\mathrm{pH}$ from sample to sample. Throughout the groundwater sampling campaign, the $\mathrm{pH}$ and the temperature of the well water varied by only about $0.5 \mathrm{pH}$ units and about $5^{\circ} \mathrm{C}$.

Stream flow rates calculated at the mouth of Mosquito Creek and near the Trench Warfare Catch Box showed significant variation with the seasons and also with the daily tide cycles (Table 3-9). The flow rate at the mouth of Mosquito Creek on the morning of September 10, 1993, was almost an order of magnitude greater than that in the afternoon, between the tides. Flow after a high tide reflects drainage of the water added to the channel during high tide, and the flow in the period between tides reflects base flow of the stream. Stream flow near the headwaters could not be measured in April 1993 because of overgrowth in the channel that nearly stopped all flow.

Uranium concentrations in the surface and groundwater samples are shown in Table 3-10 and in Appendix C (Table C-1). None of the water samples analyzed had ${ }^{235} \mathrm{U}$ or ${ }^{234} \mathrm{U}$ in concentrations greater than detection limits. Therefore no isotopic ratios could be computed, and it was not possible to attribute the source of uranium to DU or natural U. ${ }^{238} \mathrm{U}$ concentrations above detection limits in groundwater samples ranged from $1.1 \times 10^{-2} \mathrm{pCi} / \mathrm{L}$ to $3.3 \times 10^{-1} \mathrm{pCi} / \mathrm{L}$. These concentrations are well below the nationwide mean $U$ concentration of $2 \mathrm{pCi} / \mathrm{L}$ for groundwaters reported by Cothern and Lappenbusch (1983) and are most probably due to the weathering of natural $U$ in the soils and rock formations of the area. One $U$ concentration from surface water was significantly greater than the groundwater concentrations, one was approximately equal to the highest groundwater measurement, and the concentrations of the remaining surface water samples were below the detection limit. The sample from Mosquito Creek near the catch box had the largest ${ }^{238} \mathrm{U}$ concentration of $3.4 \mathrm{pCi} / \mathrm{L}$. However, no ${ }^{235} \mathrm{U}$ was detected in that sample, and the isotopic ratio could not be calculated. A large penetrator fragment was found about $10 \mathrm{~m}$ upstream from the collection point, and the sampling site itself is on the firing line of the Main Front Firing Range, about $50 \mathrm{~m}$ downrange from the 4000-m target. Samples from the mouth of Mosquito Creek showed low concentrations of $U$. 
Table 3-6. Water quality data for surface water collected in July 1992 at APG. ND indicates no data collected.

\begin{tabular}{|c|c|c|c|c|c|c|c|}
\hline SITE & $\begin{array}{c}\text { Temperature } \\
\left({ }^{\circ} \mathrm{C}\right)\end{array}$ & $\mathbf{p H}$ & $\begin{array}{c}\text { Dissolved } \\
\text { Oxygen } \\
\text { (mg/L) }\end{array}$ & $\begin{array}{c}\text { Salinity } \\
(\%)\end{array}$ & $\begin{array}{c}\text { Conductivity } \\
\text { ( } \mu \text { mhos })\end{array}$ & $\begin{array}{c}\text { Depth } \\
\text { (m) }\end{array}$ & $\begin{array}{c}\text { Visibility } \\
\text { (m) }\end{array}$ \\
\hline $\begin{array}{l}\text { Delph } \\
\text { Creek \#1 }\end{array}$ & 22.0 & 7.04 & 5.5 & 0.5 & 975 & 1.2 & 0.4 \\
\hline $\begin{array}{l}\text { Delph } \\
\text { Creek \#2 }\end{array}$ & 22.5 & 6.96 & 6.0 & 0.75 & 1100 & 0.8 & 0.4 \\
\hline $\begin{array}{l}\text { Upper } \\
\text { Delph } \\
\text { Creek }\end{array}$ & ND & 5.07 & ND & 0 & 440 & ND & ND \\
\hline $\begin{array}{l}\text { Upper } \\
\text { Mosquito } \\
\text { Creek }\end{array}$ & 20.0 & 5.99 & 5.2 & 0 & 70 & ND & ND \\
\hline $\begin{array}{l}\text { Mosquito } \\
\text { Creek \#1 }\end{array}$ & 23.2 & 7.07 & 6.5 & 0.5 & 925 & 0.4 & 0.3 \\
\hline $\begin{array}{l}\text { Mosquito } \\
\text { Creek \#2 }\end{array}$ & 23.5 & 7.40 & 7.0 & 0.5 & 1025 & 1.4 & 0.4 \\
\hline $\begin{array}{l}\text { Old } \\
\text { Woman's } \\
\text { Gut \#1 }\end{array}$ & 22.5 & 7.18 & 6.9 & 0.5 & 910 & 0.6 & 0.4 \\
\hline $\begin{array}{l}\text { Old } \\
\text { Woman's } \\
\text { Gut \#2 }\end{array}$ & 23.2 & 7.53 & 7.5 & 0.5 & 850 & 1.5 & 0.4 \\
\hline B3 Creek & 23.0 & 6.00 & 6.5 & 0 & 62 & ND & ND \\
\hline DU Road & 23.0 & 5.77 & 4.0 & 0 & 40 & ND & ND \\
\hline $\begin{array}{l}\text { B3 Catch } \\
\text { Box }\end{array}$ & ND & ND & ND & ND & ND & ND & ND \\
\hline
\end{tabular}


Table 3-7. Water quality data for surface water collected in October 1992 at APG. ND indicates no data collected.

\begin{tabular}{|l|c|c|c|c|c|c|c|}
\hline \multicolumn{1}{|c|}{ Site } & $\begin{array}{c}\text { Temperature } \\
\left({ }^{\circ} \mathbf{C}\right)\end{array}$ & $\mathbf{p H}$ & $\begin{array}{c}\text { Dissolved } \\
\text { Oxygen } \\
(\mathbf{m g} / \mathrm{L})\end{array}$ & $\begin{array}{c}\text { Salinity } \\
(\%)\end{array}$ & $\begin{array}{c}\text { Conductivity } \\
(\mu \mathbf{m h o s})\end{array}$ & $\begin{array}{c}\text { Depth } \\
(\mathbf{m})\end{array}$ & $\begin{array}{c}\text { Visibility } \\
(\mathbf{m})\end{array}$ \\
\hline Delph Creek \#1 & 17.0 & $\mathrm{ND}$ & 10.0 & 2.2 & 2950 & 1.7 & 0.9 \\
Delph Creek \#2 & 17.0 & $\mathrm{ND}$ & 10.8 & 2.1 & 3600 & 1.1 & 1.0 \\
Upper Delph Creek & 16.0 & 5.0 & 8.4 & $\mathrm{ND}$ & 1900 & $\mathrm{ND}$ & $\mathrm{ND}$ \\
Mosquito Creek \#1 & 18.0 & 4.9 & 10.0 & 0.05 & 1000 & 0.7 & 0.4 \\
Mosquito Creek \#2 & 18.5 & $\mathrm{ND}$ & 11.4 & 0.8 & 1100 & 1.2 & 0.4 \\
Old Woman's Gut \#1 & 17.0 & $\mathrm{ND}$ & 9.6 & 2.0 & 3300 & 0.65 & 0.65 \\
Old Woman's Gut \#2 & $\mathrm{ND}$ & $\mathrm{ND}$ & $\mathrm{ND}$ & $\mathrm{ND}$ & $\mathrm{ND}$ & $\mathrm{ND}$ & $\mathrm{ND}$ \\
B3 Creek & $\mathrm{ND}$ & $\mathrm{ND}$ & $\mathrm{ND}$ & $\mathrm{ND}$ & $\mathrm{ND}$ & $\mathrm{ND}$ & $\mathrm{ND}$ \\
\hline
\end{tabular}

Table 3-8. Data from wells collected 4/4/93.

\begin{tabular}{|c|c|c|c|c|c|c|}
\hline Site & $\begin{array}{c}\text { Temperature } \\
\left({ }^{\circ} \mathbf{C}\right)\end{array}$ & $\mathbf{p H}$ & $\begin{array}{c}\text { Dissolved } \\
\text { Oxygen } \\
(\mathbf{m g} / \mathbf{L})\end{array}$ & $\begin{array}{c}\text { ORP } \\
(\mathbf{m V})\end{array}$ & $\begin{array}{c}\text { Alkalinity } \\
(\mathbf{m g} / \mathbf{L})\end{array}$ & $\begin{array}{c}\text { Depth }^{\mathrm{b}} \\
(\mathbf{m})\end{array}$ \\
\hline B-3-1 & 8 & 5.6 & 4.8 & +209 & nd & 1.2 \\
B-3-CB1 & 8 & 5.4 & 5.5 & +175 & 34 & 0.8 \\
B-3-CB2 & 8.7 & 5.3 & 1.9 & +195 & 20 & 2.1 \\
B-3-CB3 & 8.7 & 5.2 & 7.7 & +212 & 25 & 2.4 \\
\hline
\end{tabular}

a ORP is the oxidation-reduction potential. Lower values correspond to reducing environments, higher values to oxidizing environments.

${ }^{b}$ Depth to water surface.

Table 3-9. Flow rate data for Mosquito Creek near the Trench Warfare Catch Box and at the mouth. Volume flow rates are averages of three repeat measurements and the standard deviation is in parentheses.

\begin{tabular}{|c|c|c|c|}
\hline Channel Area $\left(\mathbf{m}^{2}\right)$ & $\begin{array}{c}\text { Volume Flow Rate, } \\
\text { Surface }\left(\mathbf{m}^{3} / \mathrm{sec}\right)\end{array}$ & $\begin{array}{c}\text { Volume Flow Rate } \\
\mathbf{1} \mathbf{~} \text { Depth }\left(\mathbf{m}^{3} / \mathbf{s e c}\right)\end{array}$ & Location \\
\hline $240^{\mathrm{a}}$ & $145(12)$ & $130(10)$ & Mouth \\
$240^{\mathrm{b}}$ & $21(3)$ & $\mathrm{ND}$ & Mouth \\
$1^{\mathrm{c}}$ & $0.03(0.002)$ & $0.02(0.003)$ & Catch Box \\
\hline
\end{tabular}

${ }^{2}$ Measurements made 9/10/93 at 8:30 am. Low tide was at 3:00 am, high tide at 10:20 am.

${ }^{b}$ Measurements made $9 / 10 / 93$ at 4:30 pm. Tide information same as for note 1. ND indicates flow data too low for measurement.

${ }^{c}$ Measurements made on 4/4/93 during low tide. Weeds clogged channel 9/11/93, so no measurements were made on that date. 
Table 3-10. Uranium concentrations in surface water and groundwater from APG. Numbers in parentheses indicate number of samples used to compute the average and standard deviations.

\begin{tabular}{|c|c|c|}
\hline \multirow[b]{2}{*}{ Site ID } & \multicolumn{2}{|c|}{ Total U Concentration } \\
\hline & Average (pCi/L) & $\begin{array}{c}\text { Standard Deviation } \\
(\mathbf{p C i} / \mathrm{L})\end{array}$ \\
\hline \multicolumn{3}{|l|}{ Groundwater } \\
\hline B-3-1 & $5.7 \times 10^{-2}(3)^{a}$ & $4.6 \times 10^{-2}$ \\
\hline B-3-2 & $3.3 \times 10^{-1}(3)^{a}$ & $2.4 \times 10^{-1}$ \\
\hline B-3-3 & $1.7 \times 10^{-1}(3)^{a}$ & $1.2 \times 10^{-1}$ \\
\hline B-3-4 & $\mathrm{nd}^{\mathrm{b}}$ & nd \\
\hline B-3-5 & $1.1 \times 10^{-2}(3)^{2}$ & $3.5 \times 10^{-3}$ \\
\hline B-3-6 & $6.2 \times 10^{-2}(3)^{a}$ & $5.1 \times 10^{-2}$ \\
\hline TW-CB1 & $3.3 \times 10^{-1}(3)^{\mathrm{a}}$ & $1.8 \times 10^{-1}$ \\
\hline TW-CB2 & $3.2 \times 10^{-1}(3)^{2}$ & $2.0 \times 10^{-1}$ \\
\hline $\begin{array}{l}\text { Surface Water } \\
\text { Pond Behind } \\
\text { B-3 Catch Box }\end{array}$ & $3.42(3)^{\mathrm{a}}$ & 1.63 \\
\hline $\begin{array}{l}\text { Mosquito Creek } \\
\text { Mouth }\end{array}$ & $\begin{array}{c}3.38 \times 10^{-1}(2)^{\mathrm{a}} \\
(1 \mathrm{BDL})\end{array}$ & $4.9 \times 10^{-2}$ \\
\hline Delph Creek & $\operatorname{BDL}(3)^{c}$ & \\
\hline Bolt Bridge & $\operatorname{BDL}(1)^{c}$ & \\
\hline $\begin{array}{l}\text { Mosquito Creek } \\
(\text { AW-1) }\end{array}$ & $\operatorname{BDL}(1)^{\mathrm{c}}$ & \\
\hline $\begin{array}{l}\text { Romney Creek } \\
(\mathrm{AW}-2)^{d}\end{array}$ & $\operatorname{BDL}(1)^{c}$ & \\
\hline $\begin{array}{l}\text { Mosquito Creek } \\
(\text { AW-3) }\end{array}$ & $\operatorname{BDL}(1)^{c}$ & \\
\hline $\begin{array}{c}\text { Pond, B-3 Line } \\
\text { of Fire, } 6000 \mathrm{~m} \\
(\mathrm{AW}-4)^{\mathrm{d}}\end{array}$ & $\operatorname{BDL}(1)^{c}$ & \\
\hline
\end{tabular}

${ }^{a}$ Data were total $\mathrm{U}$ or ${ }^{238} \mathrm{U}$ since ${ }^{235} \mathrm{U}$ or ${ }^{234} \mathrm{U}$ were below detection.

b Water sample from Well B-3-4 was cloudy due to well cement and was not analyzed.

c Sample analyzed but U concentration below detection limits (BDL).

d Reported in Ebinger et al. (1990). 


\subsubsection{Sediments}

Data from sediment samples analyzed by neutron activation are shown in Table 3-11 and in Appendix C (Table C-2). The NAA samples from Delph Creek and the mouth of Mosquito Creek appear to contain only natural U, whereas samples from DU Road and Upper Delph Creek appear to contain DU. A sample collected upstream from the mouth of Mosquito Creek may contain DU, but the isotopic ratio is inconclusive. Figure 3-5 shows the sampling locations.

Uranium concentrations measured with ICP-MS range from $6.7 \times 10^{-5} \mathrm{pCi} / \mathrm{g}$ to $4.1 \mathrm{pCi} / \mathrm{g}$ (Appendix C). Figure 3-6 shows the frequency distribution of the reported values. Forty samples analyzed by ICP-MS (82\%) had concentrations less than $0.6 \mathrm{pCi} / \mathrm{g}$, and isotopic ratios from only 12 samples analyzed by ICP-MS unambiguously indicated DU or natural U (Table 3-11 and Table C-3). The mean and median values for the samples were $3.4 \times 10^{-1} \mathrm{pCi} / \mathrm{g}$ and $9.6 \times 10^{-2} \mathrm{pCi} / \mathrm{g}$, respectively, and the mode was $9.6 \times 10^{-4} \mathrm{pCi} / \mathrm{g}$, showing that the distribution is skewed to values lower than the mean. DU was identified by analyzing isotopic ratios in samples from Upper Mosquito Creek and Upper Delph Creek, and both sites were within the impact area (Figure 3-6). Natural $U$ was identified in samples from each of the sediment sites (Figure 3-5).

Table 3-11 shows large differences in the concentrations of $U$ or DU in samples analyzed by NAA and ICP-MS. The results from ICP-MS are lower than the NAA results from similar samples by up to a factor of 10. The digestion of ICP-MS samples removes the $U$ and DU that are not bound in a stable form, as well as some of the $U$ or DU that is occluded in different mineral phases. At the end of the digestion, significant solid residue remained from each sample. It is probable that additional U remained in the residual solid and was not analyzed because it was not in the fraction of the sample that had dissolved. In contrast, concentrations reported in NAA results are from U or DU in the entire sample, regardless of whether or not the $U$ was in a form that could be dissolved. Thus, NAA results are expected to be greater than ICP-MS results. 
Table 3-11. Sediment samples collected at APG and analyzed by neutron activation or ICP-MS. Since ${ }^{238} \mathrm{U}$ is activated to ${ }^{239} \mathrm{U}$ in NAA, ${ }^{239} \mathrm{U}$ activities are reported instead of ${ }^{238} \mathrm{U}$. A ${ }^{235} \mathrm{U} /{ }^{339} \mathrm{U}$ activity ratio less than 0.8 for NAA data indicates the presence of DU, whereas values greater than 1.0 indicate natural uranium. In the ICP-MS data, $a^{235} \mathrm{U} /{ }^{238} \mathrm{U}$ mass ratio less than 0.0045 indicates the presence of DU whereas a ratio greater than 0.0065 indicates natural U. Site names in bold italics indicate that at least one sample contained DU. See Figure 3-6 for map locations of the sites. ND indicates that insufficient data were collected to calculate a meaningful ratio.

\begin{tabular}{|c|c|c|c|}
\hline SITE & U Concentration (pCi/g) & ${ }^{235} \mathbf{U} /{ }^{238,239}$ Ratio & No. of Samples \\
\hline \multicolumn{4}{|l|}{ NAA Data } \\
\hline Mosquito Creek \#1 & 1.8 & 0.8 & 1 \\
\hline Mosquito Creek \#2 & 3.0 & 1.1 & 1 \\
\hline Delph Creek \#1 & 4.4 & 1.1 & 1 \\
\hline Delph Creek \#2 & 3.0 & 1.2 & 1 \\
\hline Upper Delph Creek & 2.8 & 0.6 & 1 \\
\hline Upper Delph Creek & 5.4 & 0.6 & 1 \\
\hline DU Road & 1.7 & 0.6 & 1 \\
\hline \multicolumn{4}{|l|}{ ICP-MS Data } \\
\hline Upper Mosquito Creek & $3.2 \times 10^{-1}$ & $\mathrm{ND}$ & 3 \\
\hline Mosquito Creek \#I & $3.2 \times 10^{-1}$ & 0.0065 to 0.0073 & 4 \\
\hline Mosquito Creek \#2 & $1.3 \times 10^{-1}$ & $\mathrm{ND}$ & 4 \\
\hline Upper Delph Creek & $6.5 \times 10^{-1}$ & 0.0050 to 0.0079 & 12 \\
\hline Delph Creek \#1 & $2.6 \times 10^{-1}$ & ND & 4 \\
\hline Delph Creek \#2 & $1.6 \times 10^{-1}$ & ND & 4 \\
\hline DU Road & $2.4 \times 10^{-1}$ & ND & 3 \\
\hline Old Woman's Gut I & $4.9 \times 10^{-1}$ & $0.0050^{\mathrm{a}}$ & 3 \\
\hline Old Woman's Gut 2 & $3.1 \times 10^{-2}$ & $\mathrm{ND}$ & 2 \\
\hline High Velocity Road & $1.3 \times 10^{-4}$ & $\mathrm{ND}$ & 1 \\
\hline
\end{tabular}

Only one reliable value calculated. 


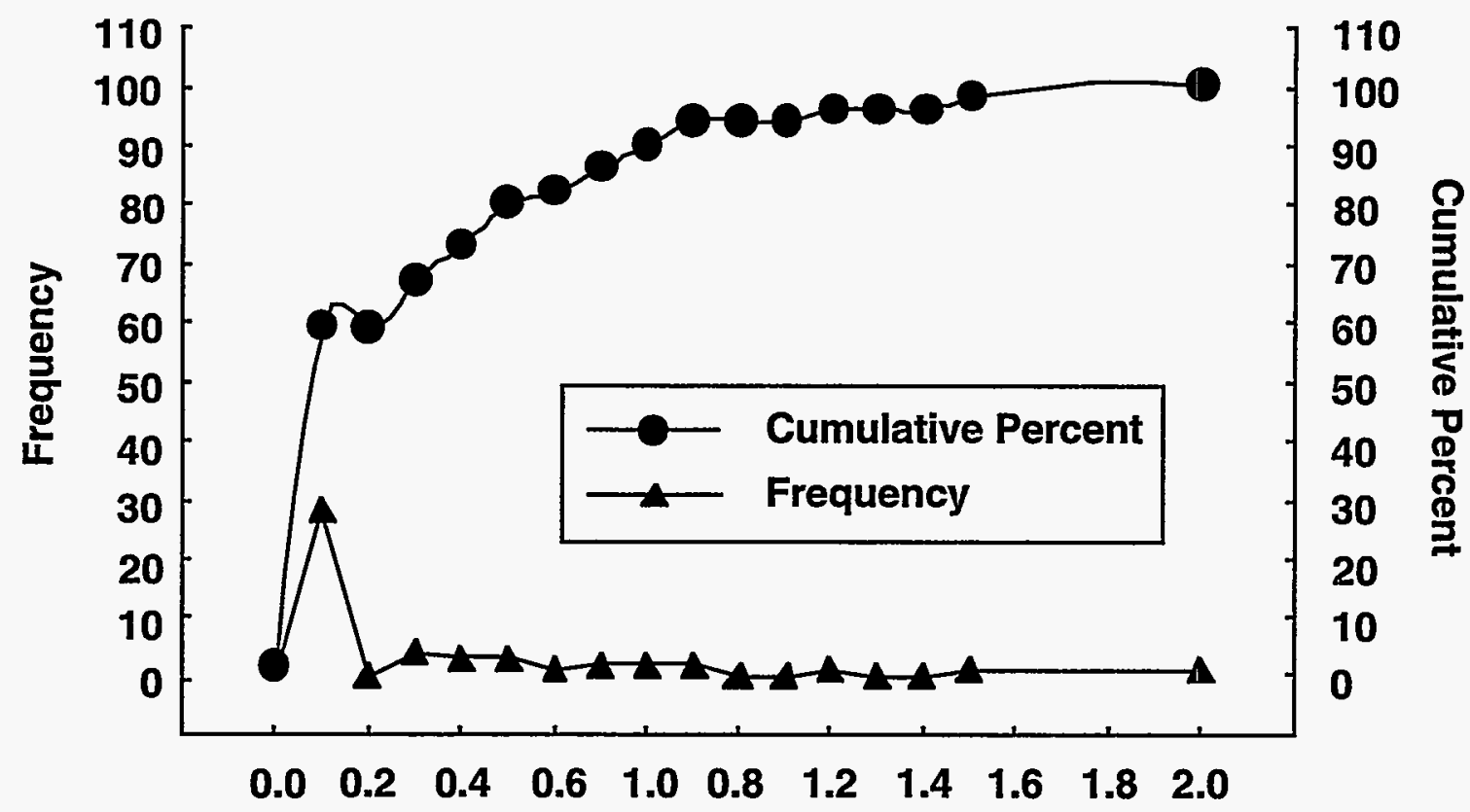

Figure 3-6. Probability distribution of U concentrations in sediments from APG. Approximately $82 \%$ of all sediment concentrations are $0.6 \mathrm{pCi} / \mathrm{g}$ or less.

\subsubsection{Aquatic Biota}

Table 3-12 and Appendix C (Tables C-3 and C-4) show the results of the $U$ analyses for aquatic animal and vegetation samples. Although DU mass per dry-tissue weight was reported for model compartments, dose calculations were made using fresh tissue masses. Doses calculated on a dry-tissue basis are $80 \%$ to $95 \%$ greater than doses to the same tissues calculated on a wet-tissue basis because of the amount of water in the wet tissues. Forty-three analyses were reported for sunfish and 131 for white perch. These sample populations were used to construct probability distributions that are shown in Figure 3-7 and Figure 3-8. Data from sunfish samples showed that $95 \%$ of the values were less than $1 \times 10^{-2}$ $\mathrm{pCi} / \mathrm{g}$ and that the samples had a mean value of $3.1 \times 10^{-3} \mathrm{pCi} / \mathrm{g}$, median of $2 \times 10^{-3} \mathrm{pCi} / \mathrm{g}$ and standard deviation of $3 \times 10^{-3} \mathrm{pCi} / \mathrm{g}$. The $95 \%$ confidence interval of the mean was $2.2 \times 10^{-3}$ to $4 \times 10^{-3} \mathrm{pCi} / \mathrm{g}$. As shown in Figure 3-7, the distribution is not normal and is skewed to the lower concentrations. Data from white perch samples showed a similar distribution with $99 \%$ of the values less than $1 \times 10^{-2} \mathrm{pCi} / \mathrm{g}$, a mean of $2.8 \times 10^{-3} \mathrm{pCi} / \mathrm{g}$, median of $2.4 \times 10^{-3} \mathrm{pCi} / \mathrm{g}$, and standard deviation of $2.2 \times 10^{-3} \mathrm{pCi} / \mathrm{g}$. The $95 \%$ confidence interval of the mean was from $2.2 \times 10^{-3} \mathrm{pCi} / \mathrm{g}$ to $3.2 \times 10^{-3} \mathrm{pCi} / \mathrm{g}$. Both data sets indicate that there is $U$ in the fish samples that is statistically greater than zero. Usually, the small concentrations of $U$ meant that the ${ }^{235} \mathrm{U}$ concentration was insufficient to calculate a reliable ${ }^{235} \mathrm{U} /{ }^{238} \mathrm{U}$ ratio. The only sunfish or perch sample that had a reliable ratio was one sunfish sample containing natural U. Five samples of various vegetation (seston, pickerel weed, millfoil, and cattails) indicated $D U$ as the source of $U$, and one Oodonta larva sample contained DU (Appendix C, Table C-1). Data from NAA showed that tadpoles collected from a puddle on DU Road about $6000 \mathrm{~m}$ downrange contained DU. Table 3-12 lists the species for which with at least one sample had an isotopic ratio suggesting the presence of DU. 
Table 3-12. Concentration of U by species from APG samples. Average and standard deviation given in $\mathrm{pCi} / \mathrm{g}$ of wet tissue. Species in bold italics indicate at least one measurement with uranium from DU. Remaining species either had insufficient data to calculate the ${ }^{235} \mathrm{U} /{ }^{238} \mathrm{U}$ ratio or the ratio indicated natural U. Additional data by species in Appendix C.

\begin{tabular}{|c|c|c|c|}
\hline Species & Average $(\mathrm{pCi} / \mathrm{g})$ & Standard Deviation (pCi/g) & $\begin{array}{c}\text { Number of } \\
\text { Measurements }\end{array}$ \\
\hline Anchovy & $1.0 \times 10^{-1}$ & $9.1 \times 10^{-2}$ & 7 \\
\hline $\begin{array}{c}\text { Blue Crab, muscle } \\
\text { Blue Crab, hepatopancreas }\end{array}$ & $\begin{array}{l}3.3 \times 10^{-2} \\
2.4 \times 10^{-2}\end{array}$ & $1.9 \times 10^{-2}$ & $\begin{array}{l}6 \\
1\end{array}$ \\
\hline Bullhead & $9.3 \times 10^{-2}$ & $2.8 \times 10^{-2}$ & 4 \\
\hline Belastoma & $9.5 \times 10^{-2}$ & & 1 \\
\hline Carp, fillets & $4.8 \times 10^{-3}$ & & 1 \\
\hline Carp, bones & $1.4 \times 10^{-1}$ & & 1 \\
\hline Carp, liver & $1.4 \times 10^{-1}$ & $7.0 \times 10^{-2}$ & 2 \\
\hline Carp, gut & $1.5 \times 10^{-2}$ & $9.2 \times 10^{-3}$ & 2 \\
\hline Catfish & $7.8 \times 10^{-3}$ & & 1 \\
\hline Cattail & 2.0 & 1.9 & 2 \\
\hline Chub & $1.4 \times 10^{-2}$ & $3.9 \times 10^{-3}$ & 2 \\
\hline Clam & $6.1 \times 10^{-2}$ & $5.0 \times 10^{-2}$ & 6 \\
\hline Crawfish & $1.1 \times 10^{-1}$ & & 1 \\
\hline Darter & $1.1 \times 10^{-2}$ & & 1 \\
\hline Eel & $4.1 \times 10^{-2}$ & & 1 \\
\hline Frog & $2.6 \times 10^{-2}$ & $3.5 \times 10^{-2}$ & 2 \\
\hline Killifish & $1.2 \times 10^{-1}$ & $1.9 \times 10^{-1}$ & 3 \\
\hline Millfoil & $4.9 \times 10^{-1}$ & $3.1 \times 10^{-1}$ & 2 \\
\hline Mosquito Fish & $8.5 \times 10^{-3}$ & & 1 \\
\hline Hogchoker & $4.2 \times 10^{-2}$ & $2.0 \times 10^{-2}$ & 7 \\
\hline Heron & $1.7 \times 10^{-3}$ & $1.8 \times 10^{-3}$ & $4^{a}$ \\
\hline Pickerel & $3.8 \times 10^{-3}$ & $1.8 \times 10^{-3}$ & 2 \\
\hline Pickerel Weed & 2.1 & & 1 \\
\hline Seston & 1.2 & $5.6 \times 10^{-1}$ & 7 \\
\hline Sunfish & $4.9 \times 10^{-2}$ & $5.2 \times 10^{-2}$ & 43 \\
\hline Shad & $6.8 \times 10^{-1}$ & $4.3 \times 10^{-1}$ & 2 \\
\hline Shrimp & $1.0 \times 10^{-1}$ & $8.9 \times 10^{-2}$ & 4 \\
\hline
\end{tabular}


Table 3-12. (cont.)

\begin{tabular}{|c|c|c|c|}
\hline Species & Average $(\mathrm{pCi} / \mathrm{g})$ & Standard Deviation $(\mathbf{p C i} / \mathrm{g})$ & $\begin{array}{c}\text { Number of } \\
\text { Measurements }\end{array}$ \\
\hline Silversides & $1.6 \times 10^{-2}$ & $4.0 \times 10^{-3}$ & 3 \\
\hline Stoneroller & $1.6 \times 10^{-1}$ & $2.5 \times 10^{-1}$ & 7 \\
\hline Tadpole & $1.8 \times 10^{-1}$ & $6.6 \times 10^{-2}$ & 5 \\
\hline Tadpole & 3.2 & & 1 \\
\hline White Perch, Whole & $5.3 \times 10^{-2}$ & $6.5 \times 10^{-2}$ & 122 \\
\hline White Perch, Liver & $8.1 \times 10^{-2}$ & $9.2 \times 10^{-2}$ & 5 \\
\hline White Perch, Fillets & $2.1 \times 10^{-2}$ & $1.3 \times 10^{-2}$ & 2 \\
\hline Yellow Perch, Whole & & 7 \\
\hline Yellow Perch, Liver & $8.1 \times 10^{-2}$ & $1.1 \times 10^{-1}$ & 1 \\
\hline Yellow Perch, Kidney & $6.8 \times 10^{-2}$ & & 1 \\
\hline
\end{tabular}

a One individual sampled, average concentration of various tissues.

b Whole samples included carcass samples (1) and fillets (2).

c Data from neutron activation, samples from ponded water on DU Road (Map location in Figure 3-6).

The biotic data were sorted into compartments according to Figure 3-1. Table 3-13 shows that the highest concentrations are measured in the detritus compartment. Ebinger et al. (1990) showed that the highest water concentration of $U$ measured from the material was that retained on $0.45 \mu \mathrm{m}$ filters, suggesting that detritus is an important transport mechanism for DU. The remaining compartments show $\mathrm{U}$ concentrations at least one order of magnitude lower than those in the detritus compartment.

Table 3-13. Uranium concentrations of various compartments of the aquatic model. Average and standard deviation given in $\mathrm{pCi} / \mathrm{g}$ of wet tissue. Bold italics indicate DU identified in at least one sample.

\begin{tabular}{|c|c|c|c|}
\hline Compartment ID & Average $(\mathbf{p C i} / \mathrm{g})$ & $\begin{array}{c}\text { Standard Deviation } \\
(\mathbf{p C i} / \mathbf{g})\end{array}$ & $\begin{array}{c}\text { Number of } \\
\text { Measurements }\end{array}$ \\
\hline Water Column Detritus $\left(\mathrm{C}_{2}\right)$ & 2.4 & 1.3 & 7 \\
Sediment $\left(\mathrm{C}_{3}\right)$ & $3.6 \times 10^{-1}$ & $6.4 \times 10^{-1}$ & 42 \\
Combined Zooplankton $\left(\mathrm{C}_{5}\right)$ & $1.9 \times 10^{-1}$ & $2.4 \times 10^{-1}$ & 7 \\
Benthic Suspension Feeders $\left(\mathrm{C}_{6}\right)$ & $6.1 \times 10^{-2}$ & $5.0 \times 10^{-2}$ & 6 \\
Benthic Deposit Feeders $\left(\mathrm{C}_{7}\right)$ & $8.6 \times 10^{-2}$ & $1.2 \times 10^{-1}$ & 40 \\
Suspension Feeding Fish $\left(\mathrm{C}_{8}\right)^{\mathrm{a}}$ & $5.8 \times 10^{-2}$ & $1.0 \times 10^{-2}$ & 129 \\
Carnivorous Fish $\left(\mathrm{C}_{9}\right)^{\mathrm{a}}$ & $4.7 \times 10^{-2}$ & $5.2 \times 10^{-2}$ & 74 \\
\hline
\end{tabular}

${ }^{2}$ White perch are included in compartments $\mathrm{C}_{8}$ and $\mathrm{C}_{9}$ in this table. 


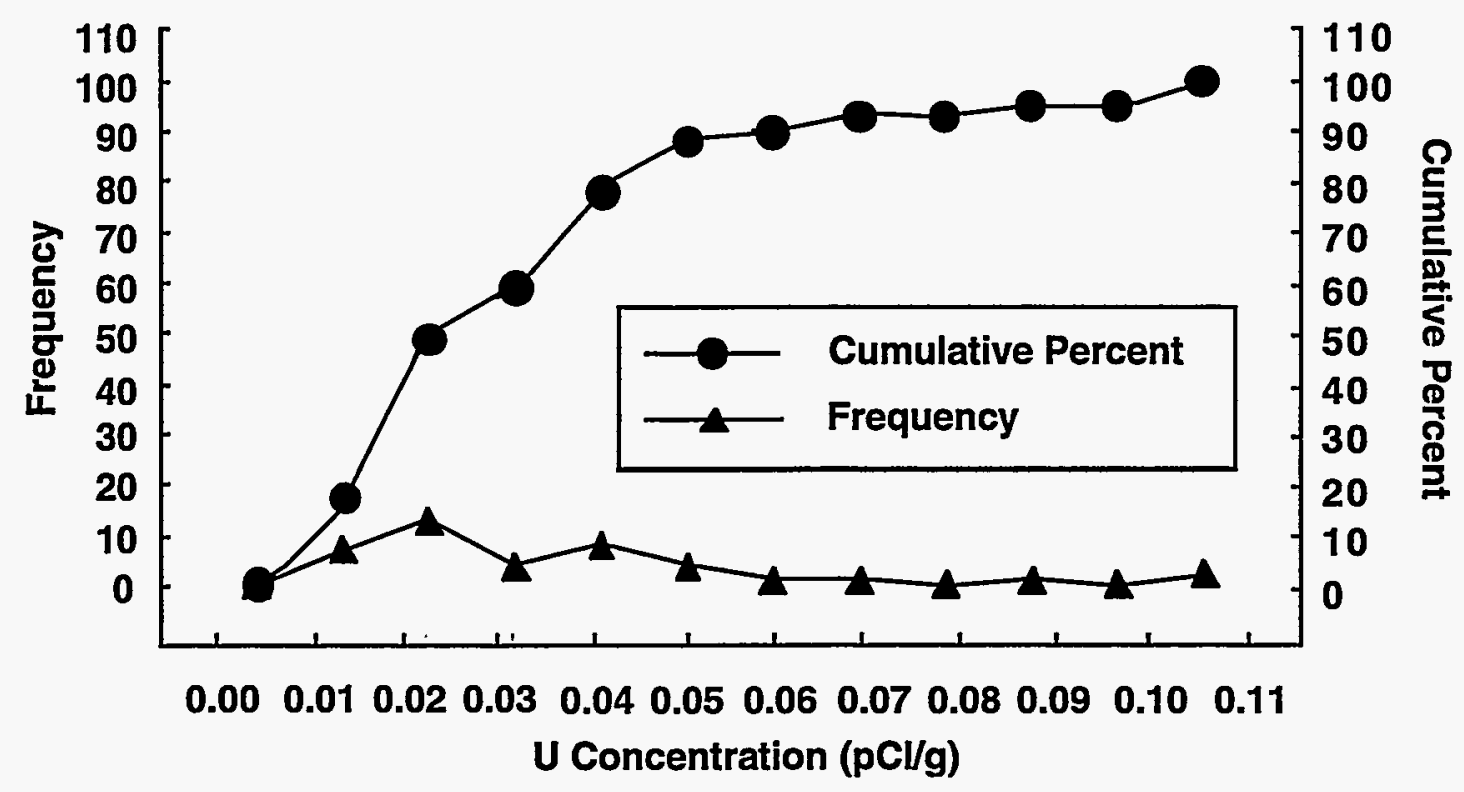

Figure 3-7. Probability distribution of U concentrations in sunfish samples from APG.

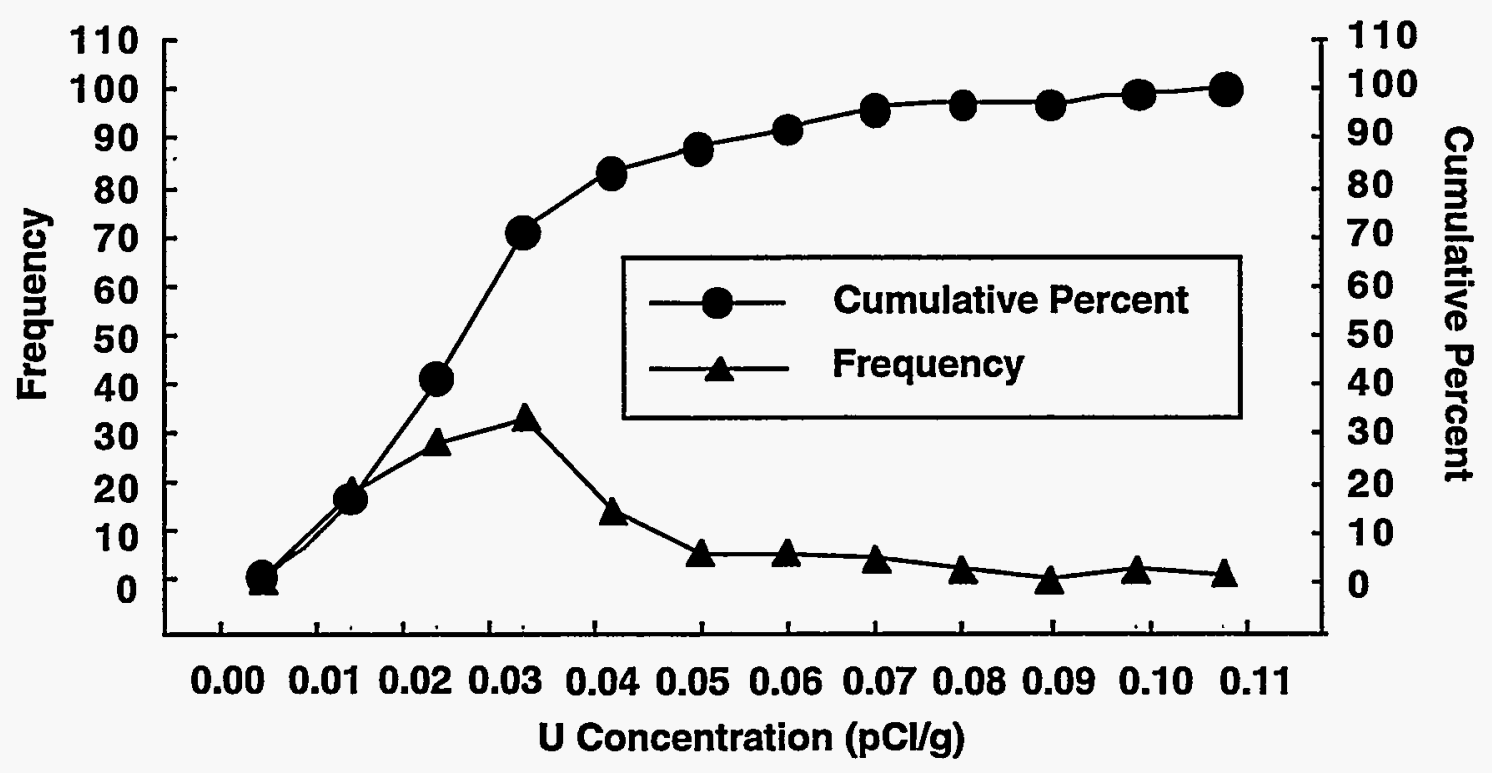

Figure 3-8. Probability distribution for U concentrations in perch from APG. 


\subsubsection{Deer Samples}

Thirty deer were collected for this study to evaluate potential DU uptake and transfer of DU to humans who consume deer tissue. Twenty-five deer were collected from the impact area and five were collected as a control group from the Eastern Shore of Maryland, approximately 10 miles east of APG and separated from APG by about 9 miles of open water. Probability of contamination of the Eastern Shore deer from DU testing at APG was considered extremely low. Kidney, liver, muscle, and bone samples were collected from each deer, and the deer's sex and probable age were determined in the field. The samples were dried, ashed, and analyzed by ICP-MS. Sampling was done to eliminate the possibility of contamination from the ground where the deer were harvested and to avoid cross-contamination between samples. Tools were cleaned before and after sampling each tissue and before a new deer was dissected. Data from the deer samples are tabulated in Appendix C (Table C-5).

Table 3-14 shows statistics derived from the deer data. Concentrations in kidney and bone samples were significantly higher (95\% confidence) in impact area deer than in off-site deer, whereas liver and muscle tissue samples were not significantly different in the two groups. Impact area deer were expected to show higher $U$ concentrations than off-site deer in tissues because of probable exposure to DU from penetrator testing. The data suggest that the expectation was correct, although the $U$ concentrations in the samples from the impact area were too low to determine the isotopic ratio and thus the source of the U. Therefore, caution is prescribed in the interpretation of the data in Table 3-14 because complete isotopic data were not available and because of the small number of samples from the off-site group.

The data in Table 3-14 show that deer from the impact area and from the off-site areas ingest U. In both data sets, however, the concentrations are minimal and no adverse health effects due to radiation from $U$ or DU are expected. Kidney burdens from impact area deer range from $7.2 \times 10^{4} \mu \mathrm{g}-\mathrm{U} / \mathrm{g}$-kidney to $1.6 \times 10^{-3} \mu \mathrm{g}-\mathrm{U} / \mathrm{g}$-kidney (95\% confidence interval), which suggests that deer do not ingest enough $\mathrm{U}$ or DU to cause nephrotoxic damage (Kocher 1989; Leggett 1989; SuLu and Zhao 1990). Kidney burdens ranged from $1.7 \times 10^{-5} \mu \mathrm{g}-\mathrm{U} / \mathrm{g}$-kidney to $7.0 \times 10^{-4} \mu \mathrm{g}$-U/g-kidney in the off-site deer. Since these values are significantly less that the $1 \mu \mathrm{g}-\mathrm{U} / \mathrm{g}$-kidney threshold level, nephrotoxic damage is not expected. 
Table 3-14. Data from deer sampling program. Off-site deer were collected on the Eastern Shore and in Cecil County, Maryland. Impact area deer were collected near the firing line of the Main Front Firing Range.

\begin{tabular}{|c|c|c|c|c|c|}
\hline Tissue & $\begin{array}{c}\text { Average } \\
\text { (pCi/g) }\end{array}$ & $\begin{array}{c}\text { Median } \\
\text { (pCi/g) }\end{array}$ & $\begin{array}{c}\text { Standard } \\
\text { Deviation } \\
\text { (pCi/g) }\end{array}$ & $\begin{array}{c}\text { 95\% Confidence } \\
\text { Interval (pCi/g) }\end{array}$ & $\begin{array}{c}\text { Number of } \\
\text { Samples }\end{array}$ \\
\hline Impact Area & & & & & 24 \\
Kidney & $4.3 \times 10^{-4}$ & $2.6 \times 10^{-4}$ & $4.3 \times 10^{-4}$ & $2.6 \times 10^{-4}$ to $5.9 \times 10^{-4}$ & 25 \\
Liver & $2.1 \times 10^{-4}$ & $1.3 \times 10^{-4}$ & $2.6 \times 10^{-4}$ & $1.1 \times 10^{-4}$ to $3.1 \times 10^{-4}$ & 19 \\
Muscle & $2.6 \times 10^{-4}$ & $2.0 \times 10^{-4}$ & $1.9 \times 10^{-4}$ & $1.7 \times 10^{-4}$ to $3.4 \times 10^{-4}$ & 18 \\
Bone & $2.7 \times 10^{-3}$ & $2.0 \times 10^{-3}$ & $2.0 \times 10^{-3}$ & $1.8 \times 10^{-3}$ to $3.6 \times 10^{-3}$ & \\
\hline Off-Site & & & & & \\
Kidney & $1.3 \times 10^{-4}$ & $1.8 \times 10^{-4}$ & $1.1 \times 10^{-4}$ & $6.0 \times 10^{-6}$ to $2.5 \times 10^{-4}$ & 2 \\
Liver & $4.0 \times 10^{-4}$ & $4.0 \times 10^{-4}$ & $2.4 \times 10^{-4}$ & $6.3 \times 10^{-5}$ to $7.4 \times 10^{-4}$ & 2 \\
Muscle & $1.0 \times 10^{-4}$ & nd & nd & nd & 2 \\
Bone & $3.4 \times 10^{-4}$ & $3.4 \times 10^{-4}$ & $3.0 \times 10^{-5}$ & 0 to $7.6 \times 10^{-4}$ & 2 \\
\hline
\end{tabular}

a Divide the value in $\mathrm{pCi} / \mathrm{g}$ by 0.37 to convert to $\mu \mathrm{g}-\mathrm{U} / \mathrm{g}$-tissue.

b Median, standard deviation, and confidence interval not calculated with only one sample reported.

\subsubsection{Soils}

Impact area soils were sampled several times during this study, mainly in conjunction with well sampling. Most soil concentrations fell between $0.5 \mathrm{pCi} / \mathrm{g}$ and $3 \mathrm{pCi} / \mathrm{g}$, unless penetrator fragments were observed (Table 3-15). The highest soil concentration measured during the current project was $32 \mathrm{pCi} / \mathrm{g}$ near Well B-3-6 on the Main Front Firing Range, and only three samples exceeded $3 \mathrm{pCi} / \mathrm{g}$. Previous studies (Ebinger et al. 1990) showed that soil concentrations could be as high as $4.3 \times 10^{4} \mathrm{pCi} / \mathrm{g}$ (43 $\mathrm{nCi} / \mathrm{g}$ ) when samples contained weathered DU fragments and were collected from soils in contact with penetrator fragments. Data from the current study and environmental monitoring at APG are shown in Appendix C (Table C-6).

We sampled soils from three locations: the vicinity of Wells B-3-3 and B-3-6, near the Trench Warfare Catch Box, and from a transect perpendicular to the line of fire about $6500 \mathrm{~m}$ downrange on the Main Front Firing Range. The transect was established with one boundary $100 \mathrm{~m}$ right (east) of the firing line and another $100 \mathrm{~m}$ left (west) of the firing line. Surface soils were sampled at $10-\mathrm{m}$ intervals, and results are shown in Table 3-15 and graphed in Figure 3-9. ${ }^{235} \mathrm{U}$ data from only three transect samples were sufficient for calculating ${ }^{235} U{ }^{238} U$ ratios. Natural $U$ was identified at the $20 \mathrm{~m}$ west location, whereas DU was the probable source of $U$ at $30 \mathrm{~m}$ and $100 \mathrm{~m}$ west. The highest $U$ concentrations were measured closest to the firing line on both the east and west arms of the transect, and both concentrations were less than $3 \mathrm{pCi} / \mathrm{g}$. All concentrations $20 \mathrm{~m}$ and beyond were $1 \mathrm{pCi} / \mathrm{g}$ or less. 
Concentrations in soil samples from the Trench Warfare Catch Box $(n=2)$ ranged from $1.9 \mathrm{pCi} / \mathrm{g}$ to $2.7 \mathrm{pCi} / \mathrm{g}$ and isotopic ratio analysis indicated that both contained natural $\mathrm{U}$ from the (Table 3-15). Concentrations in soil samples from near Well B-3-3 $(n=3)$ ranged from $1 \mathrm{pCi} / \mathrm{g}$ to $6.4 \mathrm{pCi} / \mathrm{g}$, and two of the three samples contained DU and the third, natural U. Concentrations in soil samples from the vicinity of Well B-3-6 $(n=2)$ ranged from $3.4 \mathrm{pCi} / \mathrm{g}$ to $32 \mathrm{pCi} / \mathrm{g}$. The larger concentration was due to $\mathrm{DU}$, as indicated by an isotopic ratio of 0.0029 , but the isotope ratio for the smaller concentration was 0.0055 and could not be attributed unambiguously to DU or natural U.

Eight additional soil samples were collected at different locations during the study. One sample collected on the slope leading to the headwaters of Mosquito Creek from the Trench Warfare Catch Box (TWCB-1, Table 3-15) had a concentration of $4.1 \mathrm{pCi} / \mathrm{g}$, which was attributable to DU $\left({ }^{235} \mathrm{U} /{ }^{238} \mathrm{U}\right.$ mass ratio of 0.0035), whereas two other samples from the same vicinity (TWCB-2, TWCB-3) had concentrations of $0.35 \mathrm{pCi} / \mathrm{g}$ and $0.44 \mathrm{pCi} / \mathrm{g}$ with ${ }^{235} \mathrm{U}$ below detection limits. Three samples were collected near the mouth of Delph Creek (DC-1 through DC-3) and had concentrations ranging from 0.3 $\mathrm{pCi} / \mathrm{g}$ to $1.5 \mathrm{pCi} / \mathrm{g}$. Only DC-1 had sufficient data to calculate the isotopic ratio, and natural $\mathrm{U}$ was the source of the $U$ in this sample. Two samples were collected near the mouth of Mosquito Creek (MC-1 and MC-2) and had concentrations ranging from 0.1 to $0.2 \mathrm{pCi} / \mathrm{g}$. Neither Mosquito Creek sample had sufficient ${ }^{235} \mathrm{U}$ for isotopic ratio calculation. 
Table 3-15. Soil concentrations of U from APG locations. Samples were collected between July 1992 and September 1993.

\begin{tabular}{|c|c|c|c|c|}
\hline Location & ${ }^{238} \mathrm{U}(\mathrm{pCi} / \mathrm{g})$ & ${ }^{235} \mathrm{U}(\mathrm{pCi} / \mathrm{g})$ & ${ }^{235} \mathrm{U} /{ }^{238} \mathrm{U}$ & Total $\mathrm{U}^{\mathrm{a}}(\mathrm{pCi} / \mathrm{g})$ \\
\hline \multirow[t]{3}{*}{ B-3-3 } & 6.29 & $1.1 \times 10^{-1}$ & $2.9 \times 10^{-3}$ & 6.4 \\
\hline & $9.9 \times 10^{-1}$ & $4.1 \times 10^{-2}$ & $6.7 \times 10^{-3}$ & 1.0 \\
\hline & 2.2 & $8.4 \times 10^{-2}$ & $6.6 \times 10^{-3}$ & 2.2 \\
\hline \multirow[t]{2}{*}{ B-3-6 } & $3.2 \times 10^{1}$ & $5.4 \times 10^{-1}$ & $2.9 \times 10^{-3}$ & 32 \\
\hline & 3.4 & $1.1 \times 10^{-1}$ & $5.5 \times 10^{-3}$ & 3.4 \\
\hline \multirow{2}{*}{$\begin{array}{c}\text { Trench Warfare } \\
\text { Catch Box }\end{array}$} & 2.6 & $1.2 \times 10^{-1}$ & $8.6 \times 10^{-3}$ & 2.7 \\
\hline & 1.9 & $8.6 \times 10^{-2}$ & $7.8 \times 10^{-3}$ & 1.9 \\
\hline TWCB-1 & 4.1 & $8.2 \times 10^{-2}$ & $3.5 \times 10^{-3}$ & 4.1 \\
\hline TWCB-2 & $4.4 \times 10^{-1}$ & $5.2 \times 10^{-2}$ & $3.5 \times 10^{-3}$ & 0.4 \\
\hline TWCB-3 & $3.5 \times 10^{-1}$ & $\mathrm{nd}^{\mathrm{b}}$ & nd & 0.4 \\
\hline DC-1 & 1.5 & $7.1 \times 10^{-2}$ & $8.0 \times 10^{-3}$ & 1.5 \\
\hline $\mathrm{DC}-2$ & $3.1 \times 10^{-1}$ & nd & nd & 0.3 \\
\hline DC-3 & $3.0 \times 10^{-1}$ & nd & nd & 0.3 \\
\hline $\mathrm{MC}-1$ & $2.0 \times 10^{-1}$ & nd & nd & 0.2 \\
\hline MC-2 & $1.0 \times 10^{-1}$ & nd & nd & 0.1 \\
\hline \multicolumn{5}{|l|}{ Transect } \\
\hline $10 \mathrm{~m}$ East & 2.8 & $6.5 \times 10^{-1}$ & $4.0 \times 10^{-2}$ & 2.8 \\
\hline $10 \mathrm{~m} \mathrm{West}$ & 2.0 & $5.1 \times 10^{-1}$ & $4.3 \times 10^{-2}$ & 2.0 \\
\hline $20 \mathrm{~m}$ East & $1.0 \times 10^{-1}$ & nd & nd & $1.0 \times 10^{-1}$ \\
\hline $20 \mathrm{~m} \mathrm{West}$ & $6.7 \times 10^{-1}$ & $2.6 \times 10^{-2}$ & $6.7 \times 10^{-3}$ & $6.7 \times 10^{-1}$ \\
\hline $30 \mathrm{~m}$ East & $1.0 \times 10^{-1}$ & nd & nd & $1.0 \times 10^{-1}$ \\
\hline $30 \mathrm{~m} \mathrm{West}$ & $3.7 \times 10^{-1}$ & $1.0 \times 10^{-2}$ & $4.8 \times 10^{-3}$ & $3.7 \times 10^{-1}$ \\
\hline $40 \mathrm{~m}$ East & $7.0 \times 10^{-2}$ & nd & nd & $7.0 \times 10^{-2}$ \\
\hline $40 \mathrm{~m}$ West & $4.1 \times 10^{-1}$ & $5.2 \times 10^{-2}$ & $2.2 \times 10^{-2}$ & $4.1 \times 10^{-1}$ \\
\hline $50 \mathrm{~m}$ East & $1.1 \times 10^{-1}$ & nd & nd & $1.1 \times 10^{-1}$ \\
\hline $50 \mathrm{~m} \mathrm{West}$ & $8.1 \times 10^{-1}$ & $1.1 \times 10^{-1}$ & $2.4 \times 10^{-2}$ & $8.1 \times 10^{-1}$ \\
\hline $60 \mathrm{~m}$ East & $1.8 \times 10^{-1}$ & nd & nd & $1.8 \times 10^{-1}$ \\
\hline $60 \mathrm{~m} \mathrm{West}$ & $2.0 \times 10^{-1}$ & nd & nd & $2.0 \times 10^{-1}$ \\
\hline $70 \mathrm{~m}$ East & $1.0 \times 10^{-1}$ & nd & nd & $1.0 \times 10^{-1}$ \\
\hline $70 \mathrm{~m}$ West & 1.0 & $1.210^{-1}$ & $2.0 \times 10^{-2}$ & 1.0 \\
\hline $80 \mathrm{~m}$ East & $1.4 \times 10^{-1}$ & nd & nd & $1.4 \times 10^{-1}$ \\
\hline $80 \mathrm{~m} \mathrm{West}$ & $5.2 \times 10^{-1}$ & $5.210^{-2}$ & $1.7 \times 10^{-2}$ & $5.2 \times 10^{-1}$ \\
\hline
\end{tabular}


Table 3-15. (cont.)

\begin{tabular}{|c|c|c|c|c|}
\hline Location & ${ }^{238} \mathrm{U}(\mathbf{p C i} / \mathrm{g})$ & ${ }^{235} \mathbf{U}(\mathbf{p C i} / \mathbf{g})$ & ${ }^{235} \mathbf{U} /{ }^{238} \mathbf{U}$ & Total U $^{\mathbf{a}}(\mathbf{p C i} / \mathbf{g})$ \\
\hline $90 \mathrm{~m}$ East & $2.4 \times 10^{-1}$ & nd & nd & $2.4 \times 10^{-1}$ \\
\hline $90 \mathrm{~m}$ West & $1.6 \times 10^{-1}$ & nd & nd & $1.6 \times 10^{-1}$ \\
\hline $100 \mathrm{~m}$ East & $1.3 \times 10^{-1}$ & nd & nd & $1.3 \times 10^{-1}$ \\
$100 \mathrm{~m}$ West & $8.1 \times 10^{-1}$ & $1.9 \times 10^{-2}$ & $4.1 \times 10^{-3}$ & $8.1 \times 10^{-1}$ \\
\hline
\end{tabular}

a Total $U$ was the sum of ${ }^{238} U$ and ${ }^{235} U$ from the previous columns.

b nd indicates no data collected and no isotopic ratio calculated.

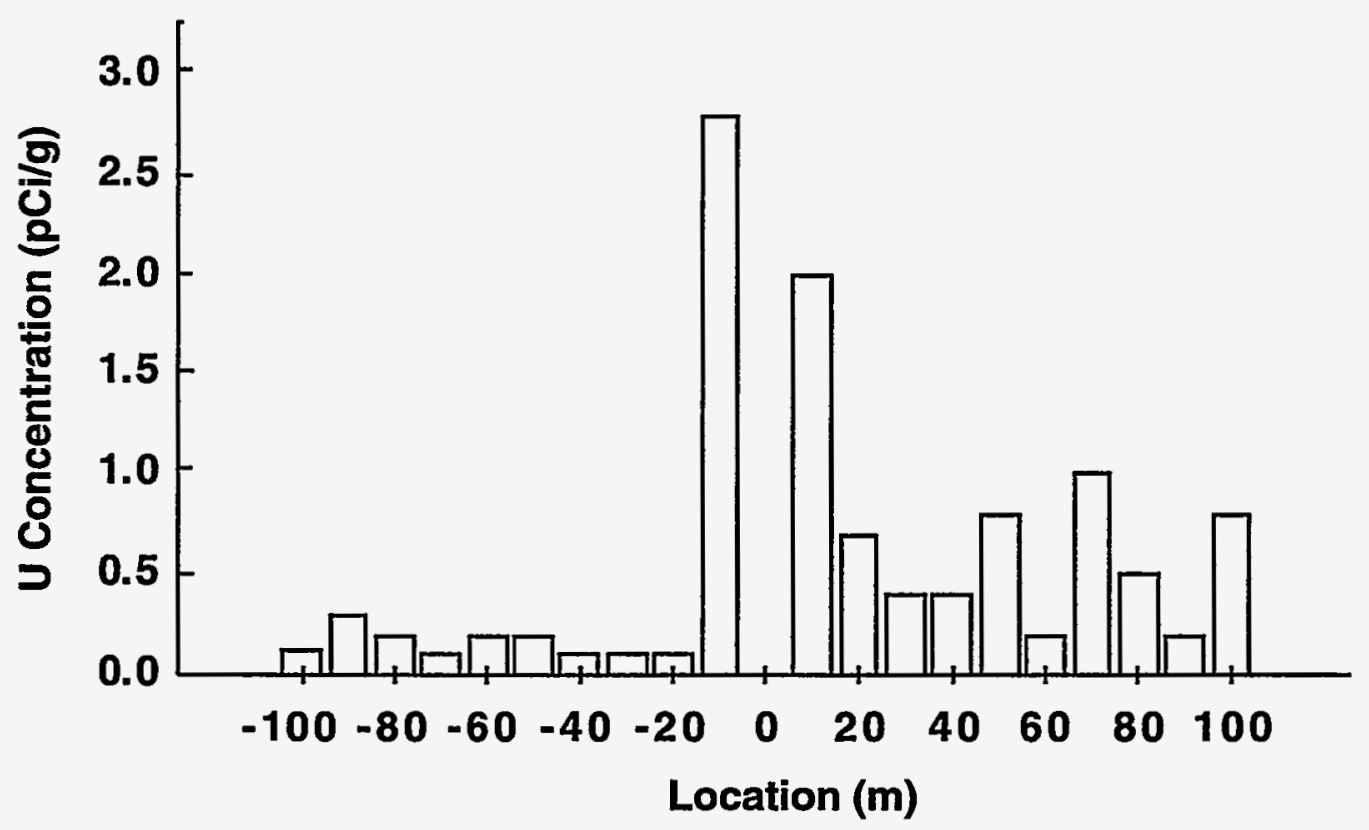

Figure 3-9. Soil transect results at $6500 \mathrm{~m}$ downrange on the Main Front Firing Range line of fire. Negative values refer to distances east (left) of the firing line, positive values refer to locations west (right) of the firing line. 
Data from environmental monitoring at APG were used to estimate the soil concentrations of $U$ at the impact area. The frequency distribution that was generated from these data is shown in Figure 3-10. About half of the 306 values reported in the environmental monitoring reports are for less than $10 \mathrm{pCi} / \mathrm{g}$, and $94 \%$ are for less than $40 \mathrm{pCi} / \mathrm{g}$. The data represent gross alpha and gross beta values and are of uncertain quality for several reasons. First, all gross alpha and gross beta activity were attributed to ${ }^{238} \mathrm{U}$. Second, no isotopic information about the $U$ in the samples was reported that could be used to account for the alpha and beta contributions specifically from ${ }^{234} U$ or ${ }^{235} U$. Third, soil moisture and density and the density of litter on the soil surface affect the gross alpha/gross beta values, and no compensation for these parameters was reported. While the environmental monitoring data are useful, their use is with the acknowledged uncertainty.

The mean of the distribution is $16.6 \mathrm{pCi} / \mathrm{g}$, the median is $8.8 \mathrm{pCi} / \mathrm{g}$, and the $95 \%$ confidence interval is 12.3 to $21 \mathrm{pCi} / \mathrm{g}$. About $64 \%$ of the samples fall below the lower bound of the $95 \%$ confidence interval because the distribution is highly skewed to values lower than the mean. While the descriptive statistics do not characterize the distribution of soil concentrations well, the distribution itself shows the large variation in soil concentration data.

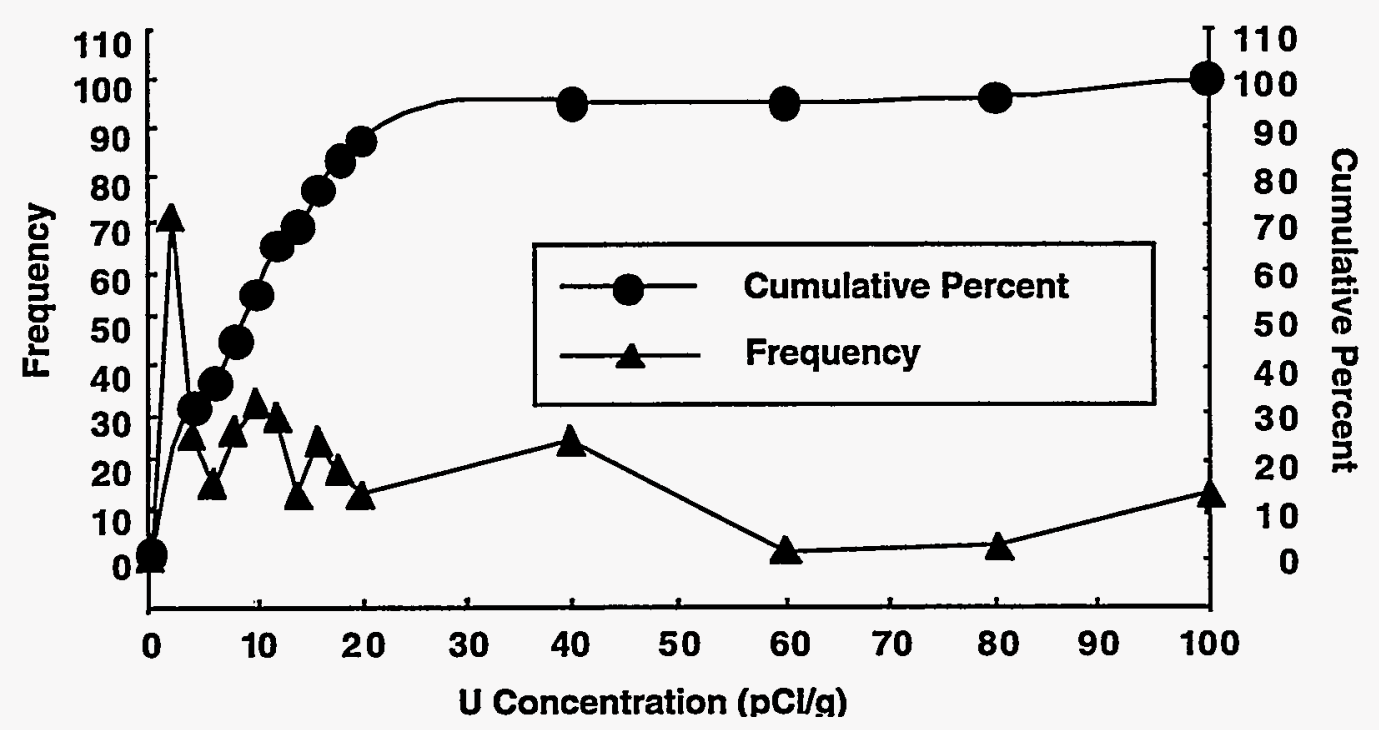

Figure 3-10. Distribution of the soil concentrations of $U$ or DU in APG environmental monitoring samples from 1980 through 1990 . See Table C-6 for concentrations $>50 \mathrm{pCi} / \mathrm{g}$. 


\subsubsection{Discussion}

Preliminary results indicate that detectable amounts of $U$ were found in many biotic samples from APG. Presence of $U$ in deer tissue and organs and in fish tissue are examples. Presence of DU could not be confirmed in any of the samples because the ${ }^{235} \mathrm{U}$ concentrations were below detection limits and the isotopic ratios could not be calculated. The $U$ concentrations in deer tissue and organs were significantly greater (95\% confidence interval) than the concentrations in off-site deer even though the concentrations in impact area deer were nearly equal to those in the off-site deer. A larger sample of deer is needed, particularly from the off-site areas, before the impact area can be shown to be the cause of the higher $U$ concentrations.

${ }^{235} U$ was not detected in most of the samples even when ${ }^{238} U$ was greater than the detection limit. Therefore, meaningful ${ }^{235} \mathrm{U} /{ }^{238} \mathrm{U}$ ratios could not be calculated, and the source of $U$ in the majority of the samples was not identified. The only confirmed DU in aquatic samples was in tadpoles collected from ponded water on DU Road. Sediment samples also contained DU, and field observations confirmed that penetrator fragments were present in the water at the sampling site. The presence of DU was confirmed by isotopic ratios in cattail, millfoil, pickerel weed, and seston samples, indicating some uptake, attachment, or adsorption of DU from water or sediments where these aquatic organisms grow. Detritus was the compartment that contained the largest concentration of confirmed DU, with concentrations averaging about $2.4 \mathrm{pCi} / \mathrm{g}$. Sufficient numbers of perch and sunfish were sampled so that probability distributions of $U$ concentrations could be derived. These distributions showed that most of the concentrations are in the $10^{-3} \mathrm{pCi} / \mathrm{g}$ range, and only three samples contained sufficient ${ }^{235} \mathrm{U}$ for the isotopic ratio to be calculated. The isotopic ratios did not indicate that DU was present, and the low concentrations of $U$ in tissue suggest that only background concentrations of $U$ are present.

$\mathrm{U}$ was detected in most sediment samples, and DU was identified in seven sediment samples. The samples that contained DU were collected from the mouth of the stream draining the Old Woman's Gut area, Upper Delph Creek inside the impact area, and Mosquito Creek downstream from the Bolt Bridge. $U$ was detected in all samples that were not identified explicitly as containing DU, and most of the concentrations measured were less than $1.5 \mathrm{pCi} / \mathrm{g}$. The data confirm that sediments may be one sink for natural $U$ as well as DU, but the data do not support sediment as the only sink for DU at APG. Transport of DU within the impact area is supported by the sediment data and from environmental monitoring data, and the data suggest that $U$ and $D U$ are in sediments at the mouths of the streams that drain the impact area. While the data suggest that minimal $U$ and possibly DU reach Chesapeake Bay by way of Mosquito Creek, Delph Creek, and Old Woman's Gut, additional sampling of the mouths of these streams and further into the Bay is recommended before definitive statements are made. The additional sampling should be conducted as part of continuing environmental radiation monitoring.

Surface water and groundwater samples indicate that extremely low concentrations of $U$ were found and ranged from $3.5 \times 10^{-3} \mathrm{pCi} / \mathrm{L}$ to $2.4 \times 10^{-3} \mathrm{pCi} / \mathrm{L}$. Samples were concentrated before analysis; otherwise, all samples would have been below detection limits. Even though samples were concentrated from large volumes of groundwater, ${ }^{235} \mathrm{U}$ concentrations were below the detection limit in all water samples and the source of the $U$ could not be confirmed. However, the concentrations of ${ }^{238} U$ reported are 
consistent with concentrations of natural $U$ in groundwater and would not result in significant health risk to humans or the ecosystem. The surface water data confirm the presence of $U$ in water draining from the Trench Warfare Catch Box and collecting downslope in a pond that forms behind the embankment. Other ponds along the firing line, as well as the creeks that run through the impact area, did not contain detectable $U$, and no water samples contained detectable ${ }^{235} \mathrm{U}$ or ${ }^{234} \mathrm{U}$. The water data do not support or refute transport of DU from soil to groundwater because the concentrations of ${ }^{235} U$ or ${ }^{234} U$ were below detection limits and isotopic ratios could not be calculated. Continued sampling through the environmental radiation monitoring program should include groundwater and surface water samples concentrated from up to $10-\mathrm{L}$ bulk volumes to address the question of DU transport by water at APG.

Soil concentrations vary greatly from the detection limit to nearly $32 \mathrm{pCi} / \mathrm{g}$ in samples collected during this study, and concentrations show a similar trend in the environmental monitoring samples. Groundwater data do not confirm or refute wide-scale transport of DU via dissolution from soils, and soil data along with field observations suggest that only a small fraction of the total inventory of DU is available for transport in the environment at APG. The materials available for transport are probably the corrosion products weathered from penetrator fragments exposed to soils and the atmosphere and particles abraded from the penetrators as they strike the ground. Most of the DU appears to be concentrated in large, visible fragments and not dispersed throughout the soils as smaller particles or dissolved species. 



\section{ECOLOGICAL STUDIES AND MODELING AT YPG}

\subsection{Introduction}

The magnitude and sources of ecological risk to the YPG environment are important parts of a comprehensive evaluation of the continued use of DU at YPG. This portion of our study of DU in the environment was initiated to examine the potential risk from DU to the terrestrial ecosystem at YPG. Although our evaluation of DU in the environment emphasized APG ecosystems over YPG ecosystems, more than 500 soil samples and approximately 300 biotic samples were collected from YPG firing lines.

DU is deposited at YPG in much the same way as at APG: penetrators pass through soft targets, impact the earth, and ricochet downrange. Penetrators or fragments ultimately come to rest either at the initial impact site or at several locations downrange. Soils become contaminated with fragments and abraded particles as penetrators impact the soil. Soil and DU particles remain in the impact trace or are ejected for varying distances, depending on particle size, projectile velocity, wind speed, and topography. Initial impacts cluster behind targets, so that contamination zones from individual impacts concentrate in small areas. After multiple impacts, the surface of hundreds to thousands of square meters is contaminated with up to hundreds of $\mathrm{mg}-\mathrm{DU} / \mathrm{kg}$-soil (Price 1991). When penetrators ricochet downrange, more impact craters and soil ejection zones are produced. Soil contamination may be lower at these sites because multiple impacts are much less likely to occur nearby. DU also is dispersed as penetrators ultimately come to rest and degrade in the environment. In situ degradation processes can produce locally high levels of soil contamination (on the scale of a few square centimeters) around the fragment. For example, Ebinger et al. (1990) detected approximately $0.5 \%$ U by mass in surface soil horizons beneath DU penetrators. Price (1991) described the spatial distribution of uranium along two firing lines in the Kofa Firing Range, which are designated GP 20 and GP 17A (Figure 4-1). Soil concentrations were generally greater at GP 17 A than at GP 20, but significant DU concentrations were observed well downrange from the initial impact area at GP 20. Uranium concentrations in most locations downrange at GP 17A and GP 20 were near background levels except in the vicinity of DU penetrators or fragments. DU was confirmed as the source of elevated uranium concentrations by isotopic ratios (Figure 4-2).

Widespread deposition of DU on the GP 17A and GP 20 firing ranges presents the potential for uptake of DU by plants and animals in the terrestrial environment at YPG. The firing lines are situated on terraces above the Lower Colorado River Valley near the transition between the Lower Sonoran Desert and the Mojave Desert. Moisture availability is low; average annual precipitation is about $90 \mathrm{~mm}$ per year and sporadic (coefficient of variation, $\mathrm{CV},=42 \%$, Figure 4-3). Precipitation is patterned into summer monsoons and winter rains (Figure 4-4). Moisture stress due to low precipitation causes primary productivity to be low relative to other environments. The sparse vegetation is dominated by creosote (Larrea tridentata) and white bursage (Ambrosia dumosa), with palo verde (Cercidium floridum and $C$. mircophyllum) found in washes where moisture availability is greater. Portions of the area are covered by desert pavement, which is almost completely lacking in vegetative cover (Brown 1982). The 
combination of aridity and sparse vegetation cause other forms of biota to be less abundant than in other communities (Polis 1991). Nevertheless, unique and complex food webs have evolved under these conditions. Considering only terrestrial vertebrates, there are some 137 families, 422 genera, and 735 species associated with the Sonoran Desert (Crosswhite and Crosswhite 1982). The biological diversity of the invertebrate fauna is even more rich (Polis 1991). This richness of ecological communities is made possible by specialized behavioral and physiological adaptations to the arid environment.

Water conservation strategies are critical factors in the success of terrestrial life at YPG. One aspect of water conservation is minimization of water loss. Animals may accomplish this using behavioral approachesto minimize the time spent in the driest, hottest microclimates. Water loss can be reduced further by physiological specialization. Kangaroo rats (Dipodomys meriamii), for example, have specialized kidneys, elongated renal papillae, and long nasal passages for countercurrent heat exchange to reduce water loss (Mares 1983). Because an early, sublethal effect of $U$ in mammals is interference with renal function (Hodge 1973), physiological water conservation processes could be adversely affected by exposure to $\mathrm{U}$ or DU, and increased risk to animals such as kangaroo rats could result.

Although many of these effects are not acutely toxic to laboratory animals in controlled, benevolent environments (Yuile 1973), minor inhibition of water conservation mechanisms could significantly affect the survival of desert organisms in the wild. Changes in survival affect population sizes and the ecological structure of the environment; changes in ecological structure affect the direction and magnitude of the flow of energy and materials in the environment, that is, its ecological function. Thus, changes in the structure or function of the YPG ecosystem could pose long-term risks to its sustainability or affect overall DU redistribution patterns.

\subsection{Approach}

Our evaluation of DU exposure to the environment at YPG was performed in parallel with the APG phase of the project. Our goal was to produce a defensible, science-based assessment of exposure and risk to terrestrial life at YPG. Understanding the distribution of DU among organisms and the risks of adverse effects to organisms at YPG is also important for assessments of risks to human health and the values that humans place on biological diversity. The common approach for the two sites was to summarize and integrate information about the ecosystem structures, the ecological functions of ecosystem components, and the physiological and toxicological properties of DU into simple ecosystem models. We used uncertainty/sensitivity analyses to identify model parameters and processes that most influenced the uncertainty in the DU exposures predicted by these models. The processes and 


\section{A. GP 20 Soils}

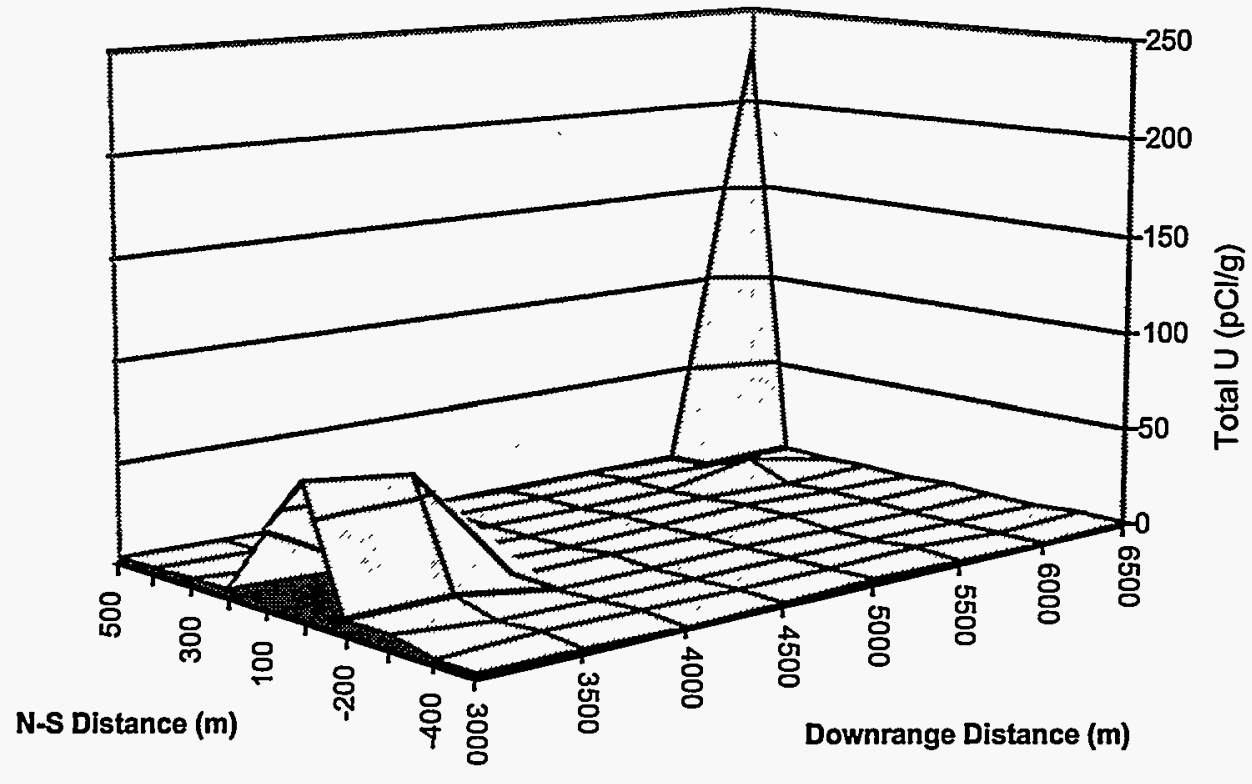

\section{B. GP 17A Soils}

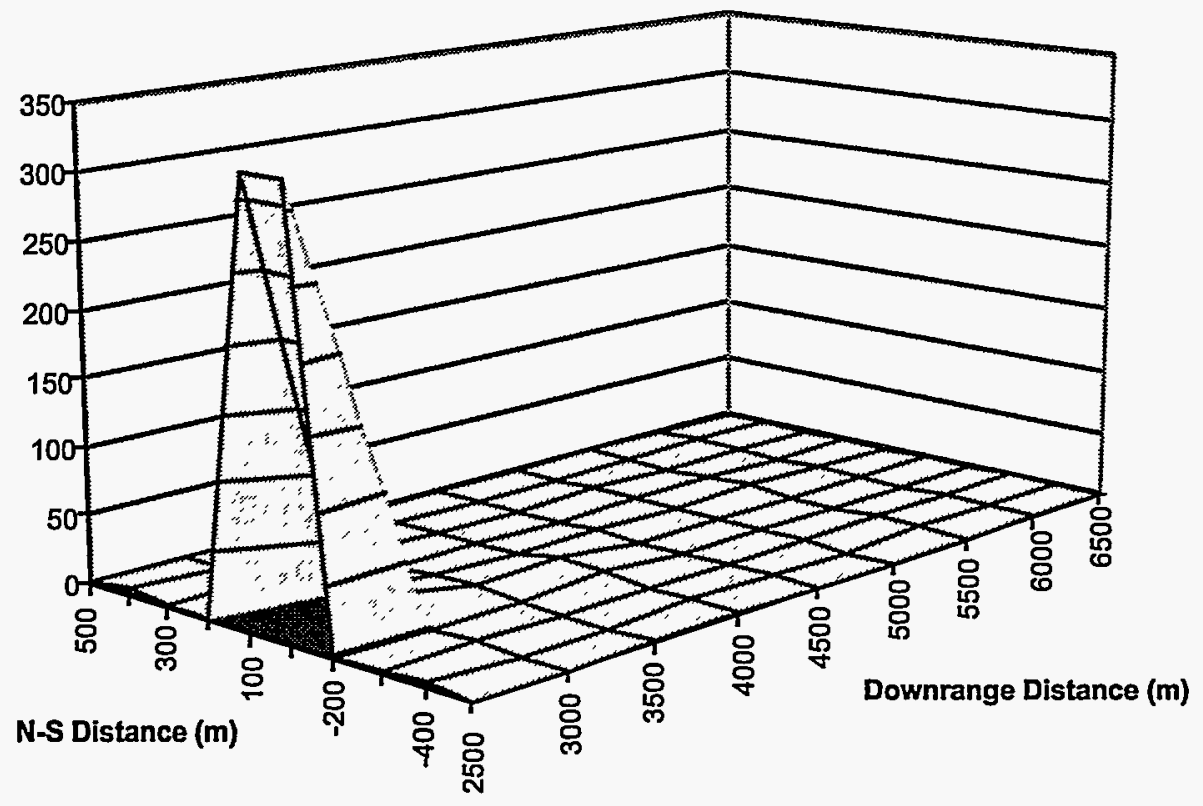

Figure 4-1. A. Spatial distribution of DU at YPG, GP 20. B. Spatial distribution of DU at YPG, GP 17A. In both diagrams, the firing line is at $0 \mathrm{~m}$, positive values on the N-S Distance axis are north of the firing line, and negative values are south of the firing line. Downrange distance is the distance from the gun position along the firing line. 


\section{A. GP 20 Soils}

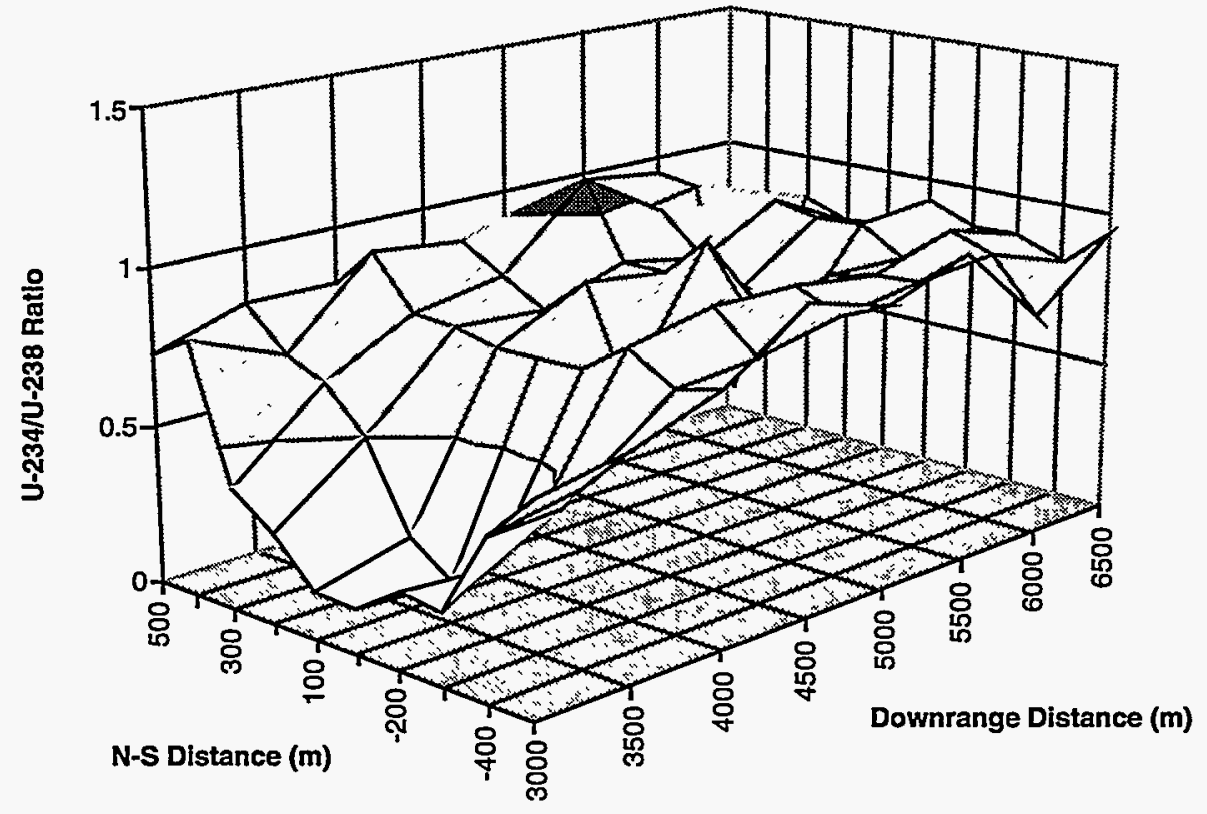

\section{B. GP 17A Soils}

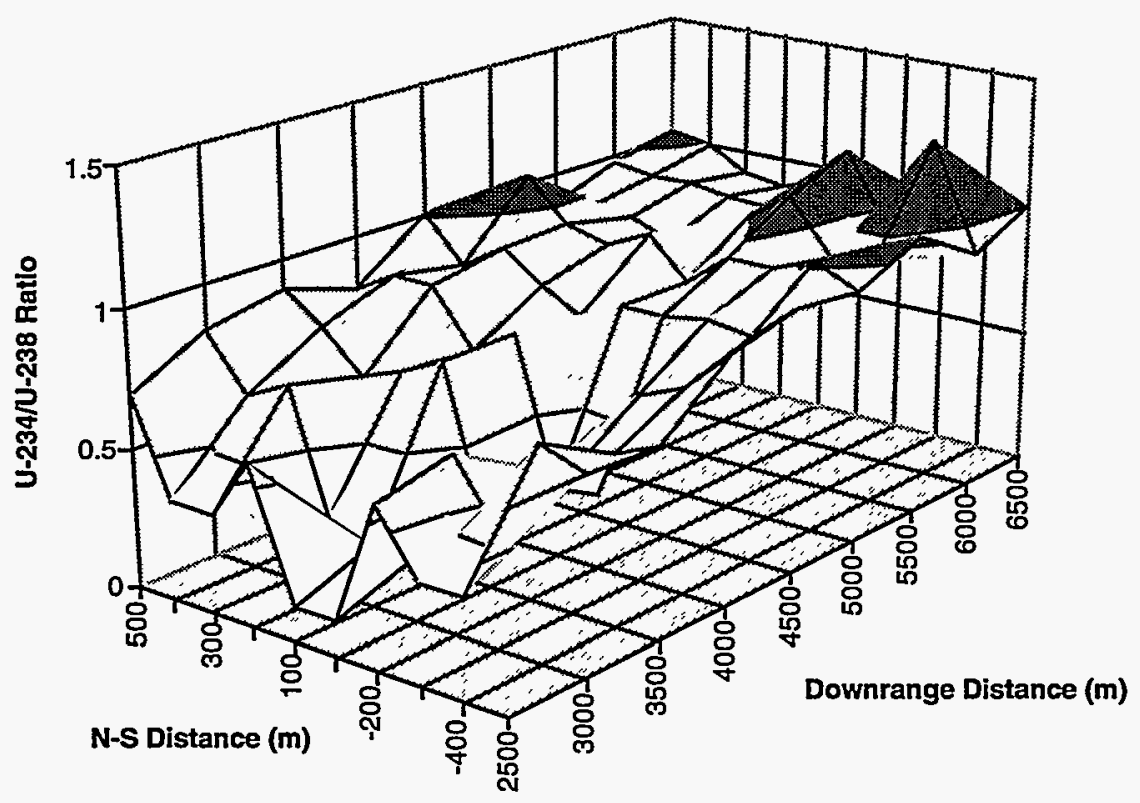

Figure 4-2. A. Spatial distribution of ${ }^{234} U{ }^{238} U$ activity ratios from GP 20, YPG. B. Spatial distribution of ${ }^{234} \mathrm{U} /{ }^{238} \mathrm{U}$ activity ratios from GP $17 \mathrm{~A}$, YPG. In both diagrams, the firing line is at $0 \mathrm{~m}$ along the N-S Distance axis, positive values are north of the firing line, and negative values are south of the firing line. Downrange distance is the distance from the gun position along the firing line. 


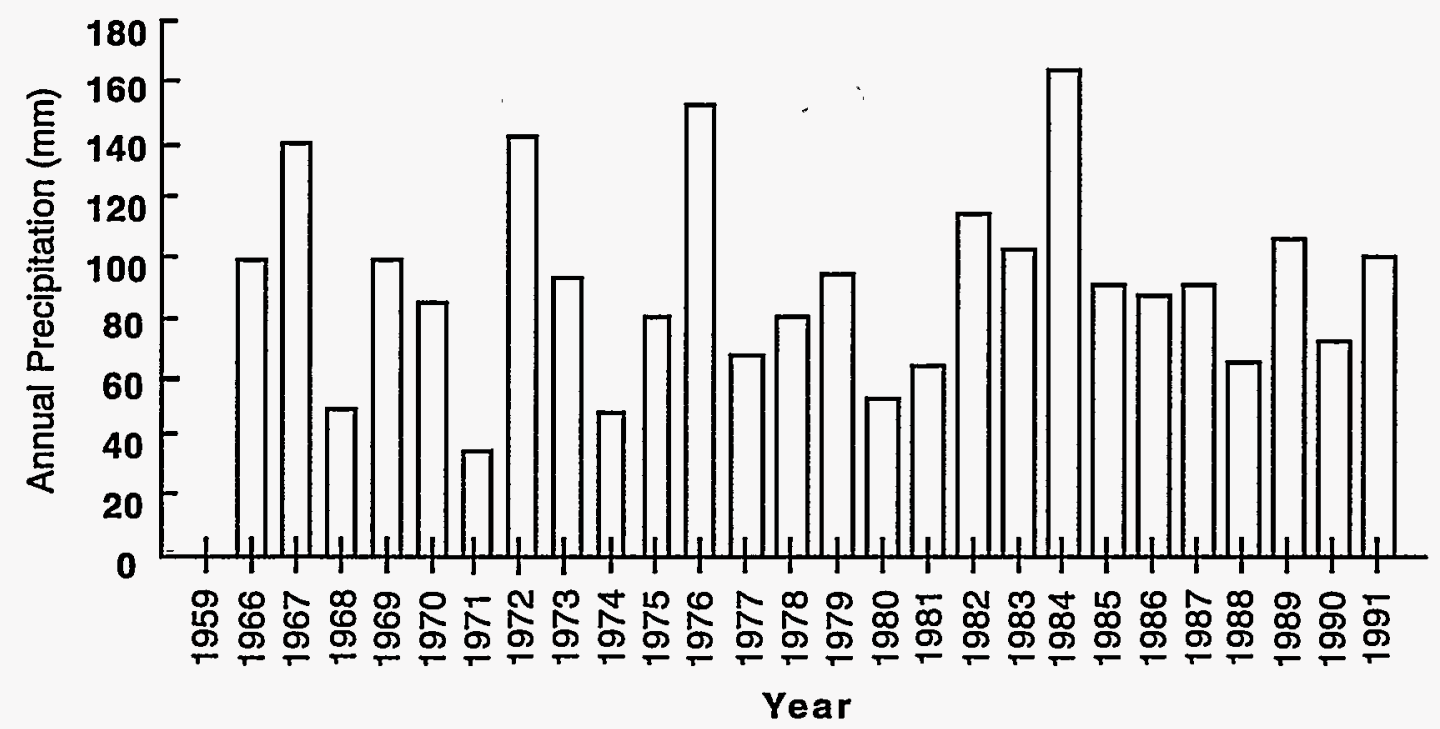

Figure 4-3. Annual precipitation at YPG, 1959 and 1966 through 1991.

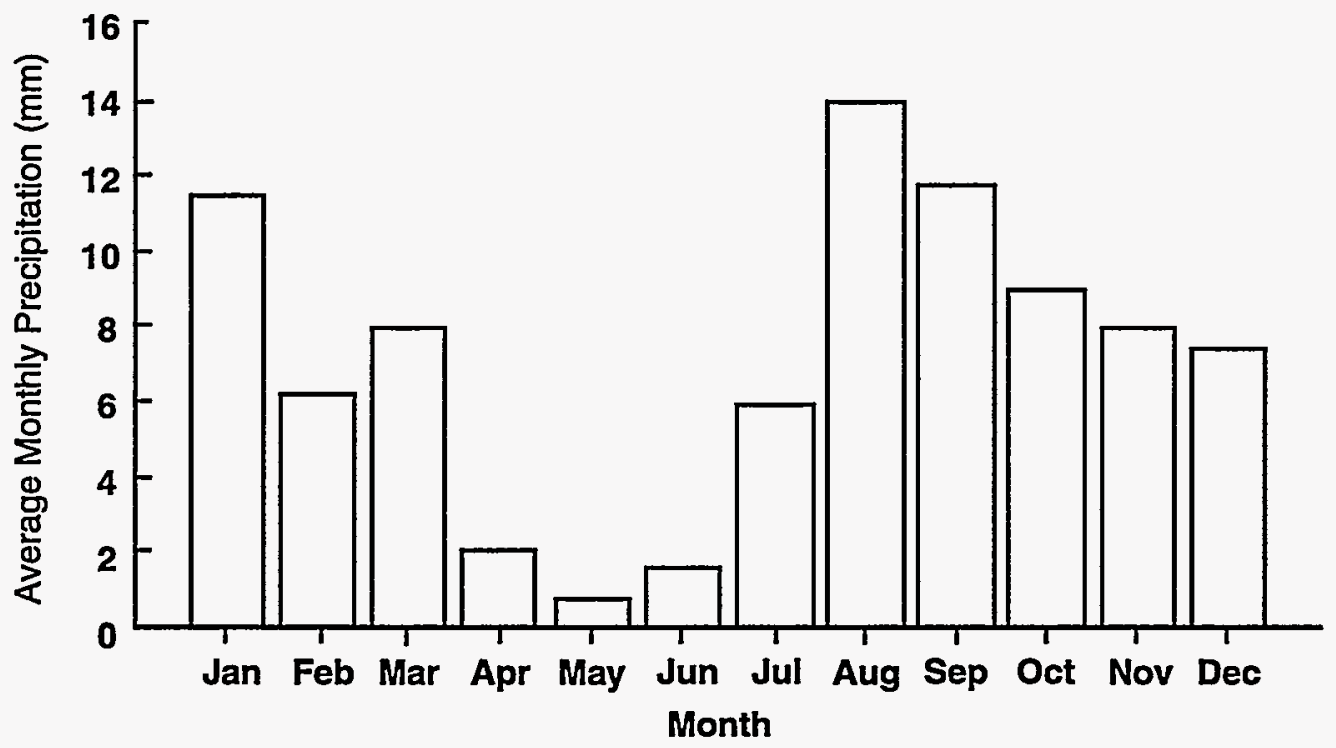

Figure 4-4. Average monthly precipitation at YPG, 1959 and 1966 through 1991. 
parameters identified in these analyses could then be targeted in subsequent field and laboratory studies to evaluate the performance of these and competing models. In August 1992 we conducted field studies to estimate the amount of DU in plants and animals at YPG. We sampled each trophic level represented in the YPG exposure model across a range of soil contamination levels so that empirical relationships between biotic and abiotic DU concentrations might be developed.

Results from samples alone provide only some of the information needed to estimate ecological exposure and risk. Animals integrate many possible exposures as they move between sites, and modes of exposure vary with time. If an adverse effect occurs, the organisms may not survive to be captured or may move to other habitats in response. These new habitats may be superior or inferior to the ones from which they moved, but the important point for the risk assessment is that the mode of exposure has changed.

We used laboratory experiments to overcome these shortcomings of field approaches. For example, we estimated the uptake and elimination rates of DU by kangaroo rats. With this information it was possible to refine predictions of DU concentrations for different exposure levels. We also estimated the histological effects that DU has on kangaroo rat kidneys because small effects on kidney function may have large overall effects on desert organisms.

\subsection{YPG Terrestrial Model}

\subsubsection{Model Structure}

We estimated the environmental fate of DU at YPG in much the same way as at APG. DU is deposited in the soil, and abiotic and biotic processes redistribute it among several compartments. We modeled these processes with coupled differential equations, and we calculated changes in the DU concentration with time in any compartment. Equation 4-1 shows the concentration in a compartment, $C_{i}$, with each time step as the sum of all inputs to $C_{i}$ from other compartments $C_{j}$ minus all losses from $C_{i \text { : }}$

$$
\frac{d C_{i}}{d t}=\frac{n}{i j}\left(C_{j} \lambda_{i j}-C_{i} \lambda_{j i}\right)
$$

where $n$ is the number of interacting compartments, $\lambda_{i j}$ is the rate of uptake (per day) to $C_{i}$ from $C_{j}$ and $\lambda_{j i}$ is the rate of loss (per day) from $C_{i}$ to $C_{j}$. Rate parameters $\left(\lambda_{i j}\right.$ and $\left.\lambda_{j i}\right)$ represent individual abiotic, ecological, or physiological processes, as well as functions of several processes, and also control flows into and out of each compartment.

We assumed in the model that DU was uniformly distributed in soils $\left(C_{1}\right)$ by the munitions testing program and that all subsequent calculations depend on this variable. The DU transport model of assumed uniform soil concentration even though uniform DU distributions are not supported by field data from YPG. We compensated for the inaccuracy of the assumption by assigning a large range of soil DU concentrations in the simulations.

Our preliminary conceptual model for DU transport and fate in the aboveground segment of the terrestrial environment at YPG contains state variables for estimating DU concentrations in plants $\left(C_{2}\right)$, 
terrestrial invertebrates $\left(C_{3}\right)$, small insectivores $\left(C_{6}\right)$, small herbivores $\left(C_{4}\right)$, large herbivores $\left(C_{5}\right)$, and predators $\left(C_{7}\right)$ (Figure 4-5). In addition, the concentration of DU in litter (detritus, $C_{8}$ ) was calculated. The litter compartment received input from the death of plants and animals and from DU in excreta. Litter was removed by consumption by invertebrates and decomposition. Rate equations used to represent DU transport in the YPG environment may be found in Table D-1 of Appendix D. Nominal values for model parameters and their ranges may be found in Table D-2.

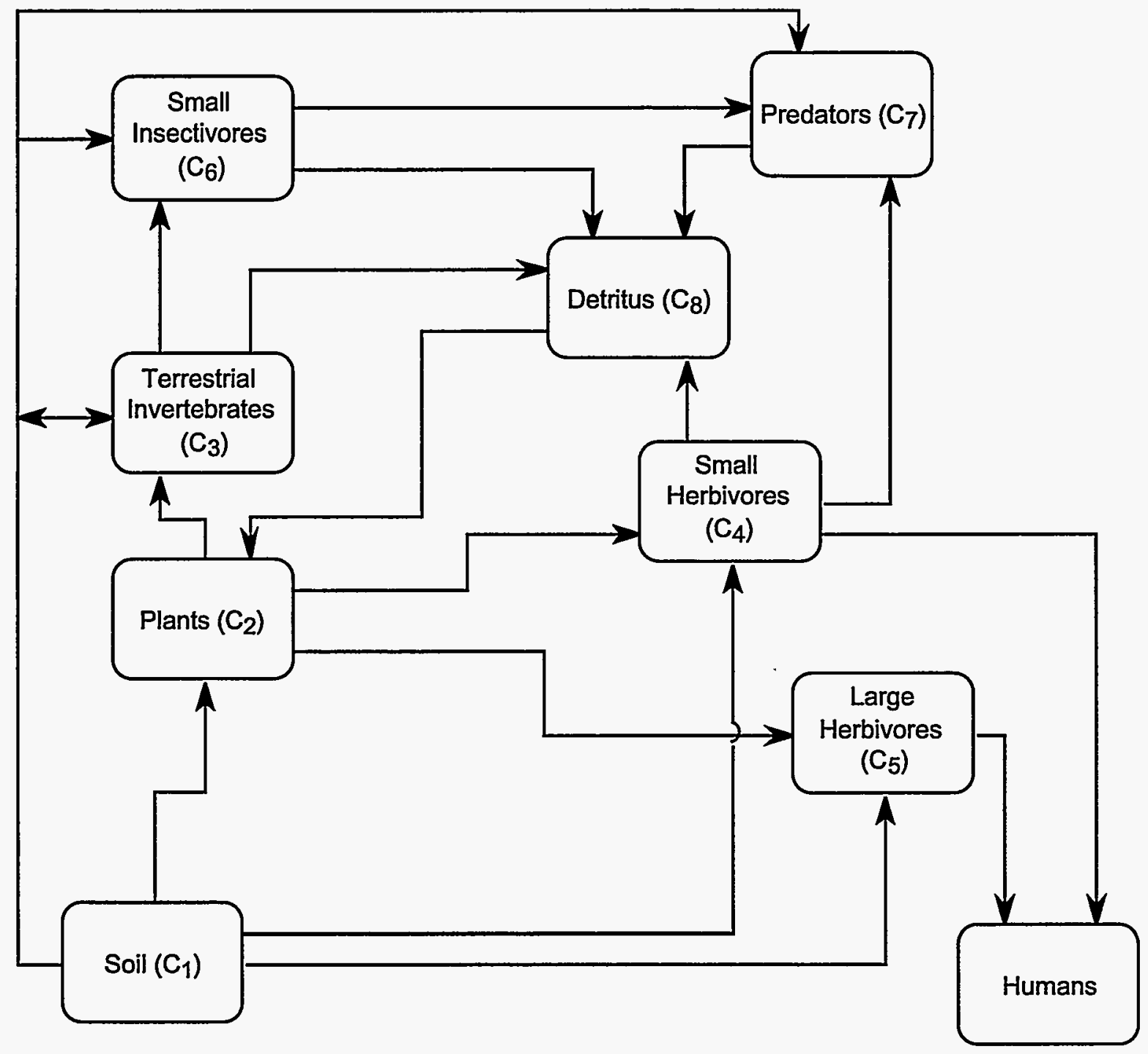

Figure 4-5. Conceptual model of DU transport through the environment at YPG. 
The species present and their abundance are important ecological factors necessary for estimating the environmental fate of DU. Ecosystem structure was determined by relationships between aboveground biomass ( $\mathrm{kg}$-dry matter $\left./ \mathrm{m}^{2}\right)$ and biomass conversion efficiencies of consumer trophic levels. Where sitespecific information was not available, the required values were estimated from the literature. We estimated aboveground plant biomass $\left(B_{2}\right)$ from literature values for the Sonoran Desert (Begon et al. 1990). We calculated biomass of invertebrates $\left(B_{3}\right)$ and large herbivores $\left(B_{5}\right)$ as fractions of aboveground plant biomass. One parameter, $B E_{\mathrm{rv}}$, represented the fraction of plant biomass contained in all herbivore compartments. A fraction of this mass was allocated to invertebrates according to the value of $\mathrm{FHV}_{3}$, and another fraction of herbivore biomass was allocated to large herbivores by $F H V_{5}$. For example, the biomass of large herbivores (in $\mathrm{kg} / \mathrm{m}^{2}$ ) was calculated as

$$
B_{5}=B_{2} B E_{\mathrm{HV}} F H V_{5}
$$

Amounts of small-herbivore biomass $\left(B_{4}\right)$ were estimated from Chew and Chew (1970). We assumed that insectivore biomass $\left(B_{6}\right)$ was equal to invertebrate biomass times the biomass conversion efficiency $\left(B E_{\mathrm{TV}}\right)$ for this group and that the biomass conversion efficiency of carnivores $\left(B E_{\mathrm{CV}}\right)$ would adequately estimate carnivore biomass as a function of total prey biomass $\left(B_{4}+B_{6}\right)$. The amount of dead plant material standing and on the soil surface was also estimated as a fraction of aboveground plant biomass $\left(B_{2}^{*} f p\right)$.

Plant uptake from soil to internal vegetation parts was calculated by

$$
C_{2,1}=C_{1} \frac{\lambda_{2,1}}{K_{\mathrm{d}}},
$$

where $\lambda_{2,1}$ was daily plant uptake and $K_{\mathrm{d}}$ was used to partition soil uranium into available and unavailable fractions.

Surface soils are available for deposition onto plant surfaces. The quantity of DU on the labile soil surface ( $Q S S, \mathrm{mg}-\mathrm{DU} / \mathrm{m}^{2}$ ) and available for suspension and rainsplash was calculated based on a labile soil depth $\left(z_{s}\right)$ of $0.001 \mathrm{~m}$, a soil concentration $\left(C_{1}\right)$, and soil bulk density $\left(P S, \mathrm{~kg} / \mathrm{m}^{3}\right)$ (Whicker and Kirchner 1987). The quantity of DU on soil surfaces was estimated by

$$
Q S S=C_{1} P S_{Z_{s}}
$$

We estimated deposition to plant surfaces by suspension and rainsplash in the manner used by Whicker and Kirchner (1987):

$$
\text { Suspension Rate }(S R)=Q S S * R F * V \text {, }
$$

and 


$$
\text { Rainsplash Rate }(R R)=Q S S k_{r}
$$

Removal of DU from plant surfaces was calculated by

$$
\text { Weathering Rate }(W R)=C_{2 S} k_{w} \quad .
$$

Uptake rates from feeding depended on daily dry matter intake and the fraction of ingested DU that was absorbed. The amount of dry matter ingested ( $D M I$, in g/day) each day by animals is related to body mass by $D M I=a W^{b}$. We estimated daily dry matter intake as a fraction of body mass $\left(F B_{i}\right)$ as

$$
F B_{i}=a_{i} W_{i}^{\left(b_{i}-1\right)}
$$

The coefficients $a_{i}$ and $b_{i}$ (Nagy 1987) were chosen based on the feeding mode of the compartments.

$D M I$ could come from one of $n$ compartments, so we partitioned DMI intake for any compartment $i$ from other compartments with the coefficient $F D_{i, j}$ such that

$$
\sum_{j=1}^{n} F D_{i j}=1
$$

Only a fraction of ingested DU is absorbed into the blood. We assumed that the assimilation coefficient $\left(E_{i, j}\right)$ was independent of the amount of intake. The amount of DU ingested with food and absorbed into the blood by large herbivores, for example, was calculated as

$$
\begin{gathered}
C_{\text {blood }}=C_{2} F B_{5} F D_{5,2} E_{5,2} \\
=C_{2} \lambda_{5,2} .
\end{gathered}
$$

Animals ingest soil deliberately or incidentally during feeding and grooming. We assumed that a fraction of daily intake was soil and that a fraction of soil DU was assimilated. Rate of daily uptake of DU from soil ingestion was estimated as

$$
\lambda_{i, 1}=f s_{i} F B_{i} E_{i, 1}
$$

where $f s_{i}$ was the fraction of daily dry matter intake that was soil.

Plants and animals lose biomass to the dead organic matter pool $\left(C_{8}\right)$ through excretion, senescence, and death. Daily mortality rates not associated with feeding by other organisms $\left(\lambda_{8, j}\right.$, per day) were derived from ( 1 - annual survival), where the survival rate $(S)$ was predicted by

$$
S=a s_{j} W_{j}^{b s_{j}}
$$

(Calder 1984). Daily loss rate from compartments due to feeding by other organisms was handled as

$$
\lambda_{j, i}=\frac{B_{j}}{B_{i}} F B_{j} F D_{j, i}
$$


Animals added DU to the dead organic matter pool $\left(C_{8}\right)$ through elimination of unabsorbed, dietary uranium at the rate $\lambda_{i^{\prime}, j}$

$$
\lambda_{i}=\frac{B_{i}^{\prime}}{B_{8}} F B_{i} F D_{i}\left(1-E_{i^{\prime}, j}\right)
$$

The urinary excretion rate to soil was calculated as a multiple $\left(F U_{i}\right)$ of the metabolic rate estimated from body mass

$$
\lambda_{1, j}=F U_{j} a m_{j} W_{j}^{b m_{j}}
$$

(Calder 1984), except for elimination by invertebrates $\left(\lambda_{1,3}\right)$. The urinary excretion rate was approximated from the four-day lead budget for Orchella cincta (van Straalen et al. 1987). The daily rate was estimated from the amount of ingested lead $\left(f_{3 i}\right)$ that was retained in the body $\left(f_{3 b}\right)$ according to

$$
f_{2 b}=f_{3 i} e^{-r t}
$$

for $t=4 \mathrm{~d}$ so that $r=\lambda_{1,3}$.

\subsubsection{Model Uncertainty and Sensitivity Analysis}

Models for estimating ecological exposures to contaminants such as DU are hypotheses about dynamic ecological processes. The ecological processes in the DU model and the parameter values used to describe these processes contain necessary uncertainties. Some of the uncertainty is a natural part of the YPG ecosystem, and some comes from incomplete knowledge of the site-specific processes and parameters that describe DU transport and fate. Our goal was to reduce this latter source of uncertainty by

- specifying a model for DU transport and fate in the YPG food web,

- analyzing the model to identify the ecological processes and parameters most influential in causing uncertainty in the predicted concentrations of DU in YPG plants and animals,

- collecting field samples from the YPG environment to estimate environmental variation in DU concentrations in the food web and to evaluate the model's structure and performance,

- experimentally estimating parameters used to describe important ecological, physiological, and toxicological processes in the model, and

- formulating a revised model with reduced uncertainty based on information gained from field and laboratory studies.

No model existed for the YPG food web or of site-specific DU transport or fate, so our model was assembled from descriptions of other sites gleaned from the scientific literature. Uncertainty in exposure models based on literature values can easily span several orders of magnitude (e.g., Lipton and Gillett 
1991), but not all model parameters and processes contribute equally. Identification of model parameters and processes most influential in creating uncertainty in estimated DU concentrations is important for understanding and managing ecological exposure and risk. Relevant field and laboratory studies directed at these factors can then determine whether the uncertainty is due to inherent variability in nature or merely due to our lack of understanding of natural processes.

Model uncertainty and parameter sensitivity of the YPG terrestrial model were tested in the same way that uncertainty and sensitivity of the APG aquatic model were tested. Basically, nominal parameter values that were best estimates of the true values were selected for the model. Ranges for each value were also selected that reflected our degree of uncertainty about the nominal values. Because no scientifically credible model existed for the biodiversity, confidence in nominal values and in the model structure were necessarily low, and parameters had broad ranges. The variation in model inputs was then analyzed to ascertain the impact each parameter had on estimated DU concentrations.

\subsubsection{Modeling Results and Discussion}

Depleted uranium concentrations predicted by the model were highly variable (Table 4-1). Plant tissues had a nominal estimated concentration of about $1 \mathrm{pCi} / \mathrm{g}$ (maximum of about 11), whereas plant surfaces contaminated by suspended DU contributed another $4 \mathrm{pCi} / \mathrm{g}$ (maximum of about 37). Except for small herbivores, the predicted concentrations for other consumers were less than or equal to about 0.4 $\mathrm{pCi} / \mathrm{g}$.

Table 4-1. Nominal and maximum DU concentrations predicted for ecosystem compartments by the YPG transport model.

\begin{tabular}{|c|c|c|}
\hline Compartment & $\begin{array}{c}\text { Nominal } \\
\text { Concentration(pCi/g) }\end{array}$ & $\begin{array}{c}\text { Maximum } \\
\text { Concentration (pCi/g) }\end{array}$ \\
\hline Plant Surfaces & 4.0 & 37 \\
Invertebrates & $4 \times 10^{-2}$ & $3 \times 10^{-1}$ \\
Small Herbivores & 4.0 & 26 \\
Larger Herbivores & $4 \times 10^{-2}$ & $7 \times 10^{-1}$ \\
Insectivores & $3 \times 10^{-1}$ & $7 \times 10^{-1}$ \\
Predators & $4 \times 10^{-1}$ & $4 \times 10^{-1}$ \\
\hline
\end{tabular}

The uncertainty in these estimates could be reduced by replacing the parameter values taken from the literature with site-specific values or by revising the model structure. An efficient approach for evaluating model structure and for refining model parameter values is to target research and sampling on ecological parameters and processes that were most influential in producing uncertainty in the model. 
Four parameters related to DU found in or on plants were important for producing uncertainty in other compartments. Suspension of DU and its subsequent deposition on plants were functions of $R F$ and $V$, and $K_{\mathrm{d}}$ controlled the amount of soil uranium that was available for uptake by plant roots. The amount of plant biomass $\left(B_{2}\right)$ also influenced model uncertainty for several reasons. First, the amount of biomass in other compartments was calculated based on the amount of plant biomass, so uncertainty in $B_{2}$ adds directly to the uncertainty of other compartments. The $B_{2}$ parameter also controls the pool of biomass that receives the suspended fraction of $D U$ and natural $U$ from the soil, and the level of contamination of plant biomass has large direct and indirect effects on DU redistribution to heterotrophs. Plant concentrations were also affected by parameters describing litter fall, plant consumption rates by invertebrates, and the biomass of vertebrate herbivores.

Predicted concentrations in invertebrates were sensitive to the amount of DU uptake directly from soil, dry matter intake rates, and invertebrates' ability to assimilate ingested DU from the detritus compartment $\left(C_{8}\right)$, in addition to being sensitive to parameters $B_{2}, R F$, and $V$. Parameters describing the DU elimination rates of large herbivores were important contributors to the uncertainty in the estimated DU content of large herbivores, and the amount of soil ingested by insectivores made a modest contribution to the uncertainty observed for predicted DU concentrations in insectivores. The uncertainty in the predicted DU concentrations in predators depended on the DU elimination rate parameters and on the DU assimilation efficiency of predators from their small-herbivore prey.

These results provide guidance for field sampling and experimental laboratory studies to evaluate model performance. Variation in soil contamination was the most important factor controlling the amount of DU available for redistribution to plants and animals. In the model, soil was deposited on plant surfaces, and then was ingested by animals or weathered from plant surfaces back to the soil. Thus, DU concentration data from plants and animals collected from areas with different soil concentrations were used to investigate empirical relationships between soil and biotic contamination levels specific to YPG. Uncertainty in some animal compartments was sensitive to DU assimilation and elimination rates, so experimental estimates of these rates should reduce overall model uncertainty. Small mammals $\left(C_{4}\right)$ were predicted to contain the greatest amounts of DU in animals, and additional research on this compartment should reduce uncertainty about ecological exposure to DU at YPG. Finally, the smallmammal communities of deserts contain important keystone species that are capable of having ecosystem-level effects not always suggested by their absolute abundance (Brown and Heske 1990). A better understanding of DU dynamics in the small-mammal community will help describe the likelihood of significant ecological effects caused by the munitions testing program at YPG.

\subsection{YPG Field Studies}

\subsubsection{Introduction}

The distribution of DU dispersed from munitions testing at YPG is not uniform, and several areas contain concentrations that are many times background levels (Price 1991). The main purpose of the YPG terrestrial model was to aid in identifying important ecological parameters and processes that limit 
our certainty about the extent of redistribution of DU from soils to the rest of the YPG ecosystem. The model was developed in the absence of detailed site-specific information on YPG ecosystem structure and function and without species-specific dose-response functions for DU. Any predictions from the model have high levels of uncertainty associated with them, though our analysis of this uncertainty has identified several of its key sources.

In this section we report results of the evaluation of the performance of the YPG terrestrial model and estimates of site-specific parameters for the model, and we discuss alternative formulations of the model. Field data are essential for evaluating performance of the YPG model and for generating relationships between DU in the abiotic environment and its redistribution to the biotic environment. Thus, we collected biological samples at YPG to estimate levels of likely exposure to DU, under the assumption that exposure was positively correlated with soil contamination.

\subsubsection{Materials and Methods}

\subsubsection{Field Sampling}

We established sample plots on two firing lines at YPG (GP 17A and GP 20 on the Kofa Firing Range). Plots were distributed nonrandomly along the firing line in areas where first penetrator impacts were closely clustered and that had been identified as having elevated levels of DU contamination (Price 1991, Figure 4-1). These areas were situated along the axis of the firing line and could be identified by impact craters, recently displaced soils, and by observation of DU fragments. Within these areas we established five sample plots on each firing line where high levels of biodiversity overlapped zones of apparent DU contamination. High biodiversity was indicated by the distribution of vegetation, animal signs, and observations of animals. These criteria resulted in plot locations in draws and washes that dissected the firing lines and in a trench along the firing line. Locations for sample plots were at different distances from the firing line and from observable impact craters and thus were assumed to cover a range of contamination levels for each firing line.

We sampled biotic and abiotic components of the YPG ecosystem at each plot. We first collected soil samples $2-3 \mathrm{~cm}$ in depth to estimate local soil contamination on the plot and then established a drift fence and pitfall trap array at that point. Individual sections of drift fences were approximately $0.15 \times$ $1.5 \mathrm{~m}$ (Figure 4-6). We operated pitfall traps for 72 hours and collected trap contents in the 2-3 hours after dawn and before dark. Using the pitfall array as the center point for the plot, we selected the nearest Larrea tridentata plant and collected samples of the surface soil and litter beneath the canopy and collected foliage samples from the canopy (Figure 4-7). Foliage samples were clipped from multiple locations in the canopy. We also identified the non-Larrea shrub or tree species nearest the plot center and collected foliage and litter samples from it by the same procedure. We sampled the grass/forb component of vegetation by collecting the aboveground biomass of the specimen nearest the plot center. We clustered Sherman live traps around each plot center in rough proportion to the abundance of burrows and other small-mammal signs, such as tracks and droppings. We operated live traps for three nights. Traps were checked in the morning, closed during the day, and opened in the evenings. In 


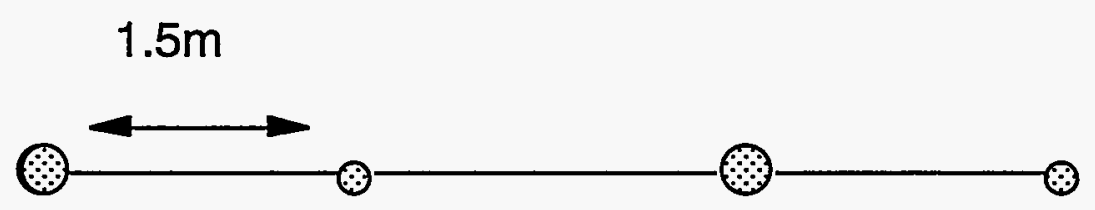

Figure 4-6. Layout of pitfall traps used to sample invertebrates, reptiles, and amphibians. Pitfall diameters were approximately $14 \mathrm{~cm}$ amd $9 \mathrm{~cm}$.

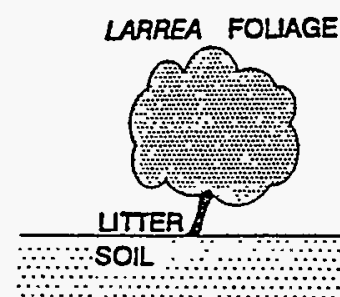

GRASS /

FORES PITFALL TRAP

SMALL MAMMALS

REPTILES

SOIL

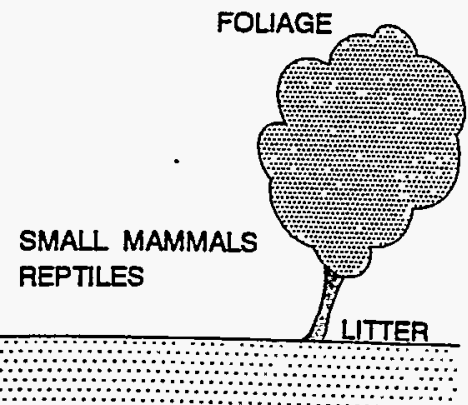

Figure 4-7. Schematic diagram of biotic and abiotic samples collected from five locations along GP 17A and GP 20 firing lines, August 1992. 
addition, we used opportunistic sampling in the vicinity of plot centers to collect snakes, lizards, rabbits, and invertebrates.

Biotic samples were placed in coolers with dry ice for storage in the field. We dissected small mammals to extract kidney and liver tissues for separate analysis. Rabbits were dissected to extract kidney, liver, and muscle samples for analysis of $U$ and DU. Tissue samples, rabbit and small-mammal carcasses, and whole bodies of other vertebrates and invertebrates were frozen in plastic bags until they could be prepared for analysis.

Sample preparation and analysis were conducted in the same manner as described previously. Biotic samples were weighed and ashed, weighed again, and then sent for analysis. Soils were dried and either extracted for IC analysis or sent to the analytical laboratory for ICP-MS analysis. No water samples were collected at YPG because no surface water was present and groundwater was about $200 \mathrm{~m}$ below the surface. Most of the YPG samples were analyzed by ICP-MS since the samples were prepared after the Omega West Reactor was taken off-line.

\subsubsection{Kangaroo Rat Histopathology}

Kidney samples from kangaroo rats were prepared for histopathology by the College of Veterinary Medicine and Biomedical Sciences Diagnostic Laboratory, Colorado State University (Fort Collins). After fixation with $10 \%$ neutral buffered formalin, sections of the kidney were placed in a tissue cassette and processed by routine histological procedures. Tissues were sequentially dehydrated in increasing concentrations of ethanol, cleared in xylene, and embedded in paraffin. Tissue sections were cut every 4$6 \mathrm{~mm}$ using a rotary microtome, stained with hematoxylin and eosin according to the Armed Forces Institute of Pathology Staining Manual (Luna 1968), flooded with mounting media, and coverslipped. Dr. David M. Getzy, Head of Pathology Services, examined the kidney tissue sections for evidence of injury and renal dysfunction.

Histopathology results from the two dosing regimes were received 15 December 1993. In the first study, tubular degeneration was seen in 4 of the 10 animals used for controls. Lesions were minimal to mild in severity, multifocal in distribution, and were nonspecific with respect to etiology. These lesions likely are incidental "background" changes, as they were not severe enough to result in clinical disease or renal dysfunction. One treatment animal $(0.65-\mathrm{mg}$ dose) showed tubular epithelial degeneration and necrosis at a severity that was indistinguishable from the controls. Significant karyomegaly in proximal tubular epithelial cells was found in three out of six high-dose animals (1.3-mg doses).

In the second study, mild lymphoplasmacytic interstitial inflammation was noted in two of the five control kidneys. These lesions were not severe enough to result in clinical disease or renal dysfunction and may be "background" changes. Two treatment animals showed tubular epithelial degeneration that was of moderate severity. The granular and hyaline degeneration of the treatment animals' tubules also were different from those of the control animals in either study. Finally, significant karyomegaly in proximal tubular epithelial cells was found in 7 of the 10 animals given $1.3 \mathrm{mg}-\mathrm{U}$ each day for three days. 
Several of the kidney lesions were as prevalent in the control animals' kidneys as in the kidneys of animals treated with uranyl nitrate. However, in the second study tubular degeneration was qualitatively different in the treatment animals from that observed in controls. In that study, kidneys of treatment animals also were more likely to exhibit significant karyomegaly in the proximal tubular epithelia. Although this a nonspecific change, it was not as striking or as severe in the control group. This change may be associated with increased ploidy of the nucleus as a result of synthesis for cell division or as a result of inhibited cytokinesis.

\subsubsection{Results from Field Studies}

We collected more than 225 plant, animal, soil, and detritus (litter) samples from YPG firing lines (Table 4-2). Invertebrates rarely contained enough mass for analysis of individuals, so individual samples were pooled according to pitfall trap site and taxonomic status. In addition, we collected 134 liver and kidney tissue samples from small mammalian herbivores and 30 liver, kidney, and muscle tissue samples from larger herbivores. Soft tissue samples were collected to allow us to estimate levels of physiological risk to these species and to provide estimates of amounts of DU that could be transferred to human consumers. Each compartment in the YPG exposure model was represented (Table 4-2), and each sample was analyzed for ${ }^{238} U$ and ${ }^{235} U$. Appendix E contains the analytical data for all samples from YPG, and Table 4-3 summarizes the data by compartment or species within a compartment.

\subsubsection{Soil Samples}

Table 4-3 shows the statistics for the soil samples at the pitfall traps and beneath the Larrea tridentata plants, and Figure 4-8 shows the distribution of the values. Five samples were in excess of $90 \mathrm{Ci} / \mathrm{g}$, and the range of concentrations measured was from the detection limit (one sample) to $518 \mathrm{pCi} / \mathrm{g}$. The range of concentrations and the distribution were expected given the nonhomogeneous deposition of the DU fragments. Isotopic ratios indicate that 2 soil samples contained primarily natural $U, 2$ samples did not contain enough ${ }^{235} U$ to calculate a reliable ratio, 16 samples contained $D U$, and 2 samples possibly contained both DU and natural U. Samples with DU ranged from $1.0 \mathrm{pCi} / \mathrm{g}$ to $518 \mathrm{pCi} / \mathrm{g}$, and samples with natural U ranged from 7.8 to $15 \mathrm{pCi} / \mathrm{g}$ (Table E-1). The confidence intervals in Table 4-3 indicate the variation in the samples, and the differences between the means and the medians for each compartment indicate the distributions are skewed significantly toward low values.

\subsubsection{Vegetation and Litter Samples}

Results for vegetation $\left(C_{2}\right)$ and litter $\left(C_{8}\right)$ in the terrestrial model (Figure 4-1) are summarized in Table 4-3. The $95 \%$ confidence interval of the $U$ concentrations on or in vegetation was $2.4 \mathrm{pCi} / \mathrm{g}$ to 19 $\mathrm{pCi} / \mathrm{g}$ (fresh weight) (Figure 4-9A; Table E-3). Vegetation samples averaged $8.1 \mathrm{pCi} / \mathrm{g}$ (fresh weight) with a median value of $1.9 \mathrm{pCi} / \mathrm{g}$ (fresh weight). DU was identified in 20 of 33 vegetation samples by isotopic ratios less than 0.0050 . Five vegetation samples contained ${ }^{238} \mathrm{U}$ concentrations in excess of 1000 $\mathrm{mg} / \mathrm{kg}$ ( $1.4 \mathrm{pCi} / \mathrm{g}$ fresh weight) and had isotopic ratios greater than 0.0050 . Of these five samples, one isotopic ratio was clearly erroneous (ratio value of 0.01121 from Sample 313), one sample (Sample 309) 
probably contained only natural $U$, and three samples (Samples 317,319 , and 324) may have contained $\mathrm{DU}$ as indicated by isotopic ratios that ranged from 0.0054 to 0.0060 . The ${ }^{235} \mathrm{U}$ concentration was below the detection limit in three vegetation samples (Samples 272, 283, and 305), and ${ }^{235} U$ in three samples was not reported (Samples 314, 315, and 316). Two vegetation samples contained $1000 \mu \mathrm{g} / \mathrm{kg} \mathrm{U}$ or less (Samples 255 and 311). No $U$ was reported for one sample of the two samples with less than $1000 \mu \mathrm{g} / \mathrm{kg}$ (Sample 255), and no ${ }^{235} \mathrm{U}$ data were reported for Sample 311. All results are listed in Table E-3.

The $95 \%$ confidence interval for litter samples was $18 \mathrm{pCi} / \mathrm{g}$ to $62 \mathrm{pCi} / \mathrm{g}$ (fresh weight) (Figure 4-9B; Table E-2). Litter samples were too brittle to wash, so only unwashed samples were analyzed. DU was identified in 16 of 18 litter samples by isotopic ratios less than 0.0050 . DU was identified in 16 of 18 litter samples by isotopic ratios less than 0.0050 . All litter samples had ${ }^{238} \mathrm{U}$ concentrations greater than $1000 \mathrm{mg} / \mathrm{kg}$ (1.4 pCi/g-fresh weight), suggesting that all 18 litter samples contained DU. Analytical error in the ${ }^{235} U$ concentrations or natural $U$ could be responsible for the isotopic ratios greater than 0.0050 in the litter samples. Results of one sample (Sample 261) suggest that analytical error was the cause of the higher isotopic ratio, results of another sample (Sample 275) suggest that natural $U$ may have been the only U present, and a third sample (Sample 326) had an erroneous isotopic ratio almost certainly due to analytical error in the ${ }^{235} \mathrm{U}$ concentration. All results are listed in Table E-2.

Ten splits of the vegetation samples were made during sample preparation to test the effects of washing on measured U concentration, and all samples were leaves or edible portions of plants. A significant difference between washed and unwashed samples would indicate U or DU on the vegetation surface, whereas no difference would indicate that uptake through roots was responsible for the observed $U$ concentration. $U$ was not detected in any of the washed splits, but $U$ was detected and DU was identified in the unwashed splits (Table E-8). We conclude that $U$ and DU were deposited on vegetation surfaces and that no significant uptake occurs through plant roots. 
Table 4-2. Numbers and types of samples collected and analyzed from YPG GP'20 and GP 17A. Each compartment of the YPG terrestrial model is represented so that redistribution of DU from soils through the food web could be estimated.

\begin{tabular}{|c|c|c|}
\hline Compartment & Samples & $\begin{array}{l}\text { Number } \\
\text { Analyzed }\end{array}$ \\
\hline Soil $\left(C_{1}\right)$ & $\begin{array}{l}\text { Pitfall trap sites } \\
\text { Sites beneath Larrea tridentata plants }\end{array}$ & $\begin{array}{l}11 \\
11\end{array}$ \\
\hline Vegetation $\left(C_{2}\right)$ & $\begin{array}{l}\text { Larrea tridentata foliage } \\
\text { Other shrubs and tree foliage (Encelia farinosa, Cercidium } \\
\text { floridum, Olynea testosa, Ambrosia dumosa, Cercidium } \\
\text { microphyllum) } \\
\text { Forb/Grass (Erioneuron pulchellum, Beloperonia } \\
\text { californica, Ditaxis sp., Euphorbia sp., Erigonum sp.) }\end{array}$ & 35 \\
\hline $\begin{array}{c}\text { Invertebrates }^{2} \\
\left(\mathrm{C}_{3}\right)\end{array}$ & $\begin{array}{l}\text { Tenebrionid beetles (Orizabus clunalis, Asbolus verracosus, } \\
\text { Asidina confluens, Cerenopus concolor, Cryptoglossa } \\
\text { muricata, Edrotes ventricosus, Eleodes longicollis), } \\
\text { Scarabaeid beetle (Orizabus clunalis), grasshoppers } \\
\text { (Acridae) crickets (Orthoptera), spiders (Araneida), ants } \\
\text { (Formicidae), scorpions (Hadrurus spadix and other } \\
\text { Scorpionidae), and mantises (Mantidae). }\end{array}$ & 45 \\
\hline $\begin{array}{c}\text { Small } \\
\text { Herbivores }\left(\mathrm{C}_{4}\right)\end{array}$ & $\begin{array}{l}\text { Pocket mice (Perognathus sp.) } \\
\text { Merriam's kangaroo rat (Dipodomys meriamii) } \\
\text { White-throated woodrat (Neotoma albigula) } \\
\text { Desert woodrat (Neotoma lepida) } \\
\text { Desert iguana (Dipsosaurus dorsalis) }\end{array}$ & $\begin{array}{c}39 \\
8 \\
14 \\
5 \\
4\end{array}$ \\
\hline $\begin{array}{c}\text { Large } \\
\text { Herbivores }\left(\mathrm{C}_{5}\right)\end{array}$ & $\begin{array}{l}\text { Desert cottontail (Sylvilagus audoboni) } \\
\text { Black-tail jack rabbit (Lepus californicus) }\end{array}$ & $\begin{array}{l}5 \\
5\end{array}$ \\
\hline $\begin{array}{c}\text { Insectivores } \\
\left(\mathrm{C}_{6}\right)\end{array}$ & $\begin{array}{l}\text { Couch's spadefoot (Scaphiopus couchii) } \\
\text { Desert horned lizard (Phrynosoma platyrhinos) } \\
\text { Long-nosed leopard lizard (Gambelia wislizenii) } \\
\text { Side-blotched lizard (Uta stansburiana) } \\
\text { Western whiptail (Cnemidophorus tigris) } \\
\text { Zebra-tailed lizard (Callisaurus draconoides) } \\
\text { small lizard (Urosaurus sp.) }\end{array}$ & $\begin{array}{c}2 \\
3 \\
1 \\
4 \\
7 \\
12 \\
1\end{array}$ \\
\hline Predators $\left(\mathrm{C}_{7}\right)$ & $\begin{array}{l}\text { Sidewinder (Crotalus cerastes) } \\
\text { Coyote (Canis latrans) scat }\end{array}$ & $\begin{array}{l}2 \\
1 \\
\end{array}$ \\
\hline Litter $\left(C_{8}\right)$ & Larrea tridentata litter, Other shrub/tree & 26 \\
\hline
\end{tabular}

${ }^{2}$ Each sample was composed of several individuals to constitute adequate mass for chemical analysis. 
Table 4-3. Descriptive statistics for U concentrations in YPG samples. All values in pCi/g except as noted for kidney samples. Sample names in bold italics indicate that DU was detected in at least one measurement. DL indicates the detection limit. Appendix E lists all data condensed in this table.

\begin{tabular}{|c|c|c|c|c|c|}
\hline Sample Type & $\begin{array}{c}\text { Mean, Total } \\
\text { U (pCi/g) }\end{array}$ & $\begin{array}{l}\text { Median, Total } \\
\text { U (pCi/g) }\end{array}$ & $\begin{array}{l}\text { Standard } \\
\text { Deviation }\end{array}$ & $\begin{array}{l}\text { 95\% Confidence } \\
\text { Interval } \\
\text { (pCi/g) }\end{array}$ & $\begin{array}{l}\text { Measurements } \\
>\text { Detection } \\
\text { Limit }\end{array}$ \\
\hline Soil & $8.3 \times 10^{1}$ & $1.6 \times 10^{1}$ & $1.5 \times 10^{2}$ & $\begin{array}{c}2.1 \times 10^{1} \text { to } \\
1.5 \times 10^{2}\end{array}$ & 22 \\
\hline Invertebrates & $3.4 \times 10^{0}$ & $3.9 \times 10^{-1}$ & $1.1 \times 10^{1}$ & $\begin{array}{l}1.7 \times 10^{-1} \text { to } \\
6.5 \times 10^{\circ}\end{array}$ & 43 \\
\hline Vegetation & $8.1 \times 10^{0}$ & $1.9 \times 10^{0}$ & $2.1 \times 10^{1}$ & $\begin{array}{c}2.4 \times 10^{0} \text { to } 1.9 \times \\
10^{1}\end{array}$ & 33 \\
\hline Litter (Detritus) & $3.7 \times 10^{1}$ & $1.3 \times 10^{1}$ & $5.3 \times 10^{1}$ & $\begin{array}{c}1.3 \times 10^{1} \text { to } 6.2 \times \\
10^{1}\end{array}$ & 18 \\
\hline $\begin{array}{c}\text { Pocket Mice } \\
\text { Carcass }\end{array}$ & $1.2 \times 10^{0}$ & $1.1 \times 10^{-1}$ & $6.4 \times 10^{0}$ & $\mathrm{DL}$ to $3.2 \times 10^{0}$ & 39 \\
\hline $\begin{array}{l}\text { Pocket Mice } \\
\text { Kidney }\end{array}$ & $4.8 \times 10^{-2}$ & $2.2 \times 10^{-2}$ & $6.0 \times 10^{-2}$ & $\begin{array}{c}1.5 \times 10^{-2} \text { to } \\
8.0 \times 10^{-2}\end{array}$ & 13 \\
\hline $\begin{array}{c}\text { Pocket Mice } \\
\text { Kidney Burden } \\
(\mu \mathrm{g} / \mathrm{g} \text {-kidney) }\end{array}$ & $1.3 \times 10^{-1}$ & $6.0 \times 10^{-2}$ & $1.6 \times 10^{-1}$ & $\begin{array}{l}4.1 \times 10^{-2} \text { to } \\
2.2 \times 10^{-1}\end{array}$ & 13 \\
\hline $\begin{array}{l}\text { Kangaroo Rat } \\
\text { Carcass }\end{array}$ & $1.4 \times 10^{-1}$ & $4.1 \times 10^{-2}$ & $1.8 \times 10^{-1}$ & $\begin{array}{c}1.9 \times 10^{-2} \text { to } \\
2.7 \times 10^{-1}\end{array}$ & 8 \\
\hline $\begin{array}{c}\text { Kangaroo Rat } \\
\text { Liver }\end{array}$ & $1.4 \times 10^{-2}$ & $9.6 \times 10^{-3}$ & $1.6 \times 10^{-2}$ & $\begin{array}{c}1.3 \times 10^{-3} \text { to } \\
2.7 \times 10^{-2}\end{array}$ & 6 \\
\hline $\begin{array}{c}\text { Kangaroo Rat } \\
\text { Kidney }\end{array}$ & $2.0 \times 10^{-1}$ & $9.8 \times 10^{-2}$ & $2.3 \times 10^{-1}$ & $\begin{array}{c}4.2 \times 10^{-2} \text { to } \\
3.6 \times 10^{-1}\end{array}$ & 8 \\
\hline $\begin{array}{c}\text { Kangaroo Rat } \\
\text { Kidney Burden } \\
(\mu \mathrm{g} / \mathrm{g}-\mathrm{kidney})\end{array}$ & $5.5 \times 10^{-1}$ & $2.6 \times 10^{-1}$ & $6.3 \times 10^{-1}$ & $\begin{array}{c}1.1 \times 10^{-1} \text { to } \\
9.8 \times 10^{-1}\end{array}$ & 8 \\
\hline $\begin{array}{l}\text { White-throated } \\
\text { Woodrat Carcass }\end{array}$ & $2.5 \times 10^{0}$ & $4.9 \times 10^{-1}$ & $6.9 \times 10^{0}$ & $\begin{array}{c}\text { DL to } \\
6.1 \times 10^{0}\end{array}$ & 14 \\
\hline $\begin{array}{l}\text { White-throated } \\
\text { Woodrat Liver }\end{array}$ & $2.7 \times 10^{-3}$ & $2.1 \times 10^{-3}$ & $2.0 \times 10^{-3}$ & $\begin{array}{c}1.5 \times 10^{-3} \text { to } \\
3.9 \times 10^{-3}\end{array}$ & 10 \\
\hline $\begin{array}{l}\text { White-throated } \\
\text { Woodrat Kidney }\end{array}$ & $2.0 \times 10^{-2}$ & $1.1 \times 10^{-2}$ & $2.4 \times 10^{-2}$ & $\begin{array}{c}6.1 \times 10^{-3} \text { to } \\
3.4 \times 10^{-2}\end{array}$ & 12 \\
\hline $\begin{array}{c}\text { White-throated } \\
\text { Woodrat Kidney } \\
\text { Burden }(\mu g / g- \\
\text { kidney })\end{array}$ & $4.3 \times 10^{-2}$ & $1.7 \times 10^{-2}$ & $5.5 \times 10^{-2}$ & $\begin{array}{c}1.2 \times 10^{-2} \text { to } \\
7.4 \times 10^{-2}\end{array}$ & 12 \\
\hline $\begin{array}{l}\text { Desert Woodrat, } \\
\text { Carcass }\end{array}$ & $3.1 \times 10^{-2}$ & $3.9 \times 10^{-2}$ & $1.6 \times 10^{-2}$ & $\begin{array}{l}1.8 \times 10^{-2} \text { to } \\
4.6 \times 10^{-2}\end{array}$ & 5 \\
\hline
\end{tabular}


Table 4-3. (cont.)

\begin{tabular}{|c|c|c|c|c|c|}
\hline Sample Type & $\begin{array}{c}\text { Mean, Total } \\
\text { U (pCi/g) }\end{array}$ & $\begin{array}{l}\text { Median, Total } \\
\text { U (pCi/g) }\end{array}$ & $\begin{array}{l}\text { Standard } \\
\text { Deviation }\end{array}$ & $\begin{array}{c}\text { 95\% Confidence } \\
\text { Interval } \\
\text { (pCi/g) }\end{array}$ & $\begin{array}{c}\text { Measurements } \\
>\text { Detection } \\
\text { Limit } \\
\end{array}$ \\
\hline $\begin{array}{l}\text { Desert Woodrat, } \\
\text { Liver }^{2}\end{array}$ & $1.1 \times 10^{-3}$ & NA & NA & $\begin{array}{c}8.5 \times 10^{-4} \text { to } \\
1.4 \times 10^{-3}\end{array}$ & 2 \\
\hline $\begin{array}{l}\text { Desert Woodrat, } \\
\text { Kidney }^{\mathrm{a}}\end{array}$ & $1.1 \times 10^{-3}$ & NA & NA & $\begin{array}{c}8.5 \times 10^{-4} \text { to } \\
1.4 \times 10^{-3}\end{array}$ & 2 \\
\hline $\begin{array}{l}\text { Desert Woodrat, } \\
\text { Kidney Burden } \\
\left(^{(\mu g / g-k i d n e y)^{a}}\right.\end{array}$ & $6.8 \times 10^{-3}$ & NA & NA & $\begin{array}{l}6.8 \times 10^{-3} \text { to } \\
6.9 \times 10^{-3}\end{array}$ & 2 \\
\hline $\begin{array}{c}\text { Desert Iguana } \\
\text { Carcass }\end{array}$ & $2.2 \times 10^{-1}$ & $2.2 \times 10^{-1}$ & $1.3 \times 10^{-1}$ & $\begin{array}{c}9.3 \times 10^{-2} \text { to } \\
3.5 \times 10^{-1}\end{array}$ & 4 \\
\hline $\begin{array}{c}\text { Desert Iguana } \\
\text { Liver }^{\mathrm{a}}\end{array}$ & $3.0 \times 10^{-1}$ & NA & NA & $\begin{array}{c}2.6 \times 10^{-3} \text { to } \\
6.0 \times 10^{-1}\end{array}$ & 2 \\
\hline $\begin{array}{c}\text { Cottontail Carcass } \\
\text { and Muscle }\end{array}$ & $7.0 \times 10^{-3}$ & $5.3 \times 10^{-4}$ & $1.1 \times 10^{-2}$ & $\begin{array}{c}\text { DL to } \\
1.6 \times 10^{-2}\end{array}$ & 6 \\
\hline Cottontail Liver & $1.1 \times 10^{-3}$ & NA & NA & $\mathrm{NA}$ & 1 \\
\hline Cottontail Kidney $^{a}$ & $1.0 \times 10^{-2}$ & NA & $8.8 \times 10^{-3}$ & $\begin{array}{c}3.8 \times 10^{-3} \text { to } \\
1.6 \times 10^{-2}\end{array}$ & 2 \\
\hline $\begin{array}{c}\text { Cottontail Kidney } \\
\text { Burden }(\mu \mathrm{g} / \mathrm{g}- \\
\text { kidney) }\end{array}$ & $2.7 \times 10^{-2}$ & NA & $2.4 \times 10^{-2}$ & $\begin{array}{c}1.0 \times 10^{-3} \text { to } \\
4.4 \times 10^{-2}\end{array}$ & 2 \\
\hline $\begin{array}{l}\text { Jack Rabbit } \\
\text { Carcass and } \\
\text { Muscle }\end{array}$ & $5.3 \times 10^{-4}$ & $3.4 \times 10^{-4}$ & $4.7 \times 10^{-4}$ & $\begin{array}{l}1.5 \times 10^{-4} \text { to } \\
9.0 \times 10^{-4}\end{array}$ & 6 \\
\hline Jack Rabbit Liver & $2.6 \times 10^{-4}$ & NA & NA & NA & 1 \\
\hline $\begin{array}{c}\text { Jack Rabbit } \\
\text { Kidney }\end{array}$ & $3.2 \times 10^{4}$ & $2.1 \times 10^{-4}$ & $2.7 \times 10^{-4}$ & $\begin{array}{c}5.2 \times 10^{-5} \text { to } \\
5.8 \times 10^{-4}\end{array}$ & 4 \\
\hline $\begin{array}{c}\text { Jack Rabbit } \\
\text { Kidney Burden } \\
\text { ( } \mu \text { g/g-kidney) } \\
\end{array}$ & $8.6 \times 10^{-4}$ & $5.5 \times 10^{-4}$ & $7.3 \times 10^{-4}$ & $\begin{array}{c}1.4 \times 10^{-4} \text { to } \\
1.6 \times 10^{-3}\end{array}$ & 4 \\
\hline Lizards & $4.0 \times 10^{-0}$ & $1.0 \times 10^{-1}$ & $1.5 \times 10^{1}$ & DL to $9.0 \times 10^{1}$ & 37 \\
\hline
\end{tabular}

${ }^{a}$ Standard deviation is actually the range of values, and the $95 \%$ confidence interval column shows the minimum and maximum of the range.

bone value omitted from this table. See Appendix E, Table E-6. 


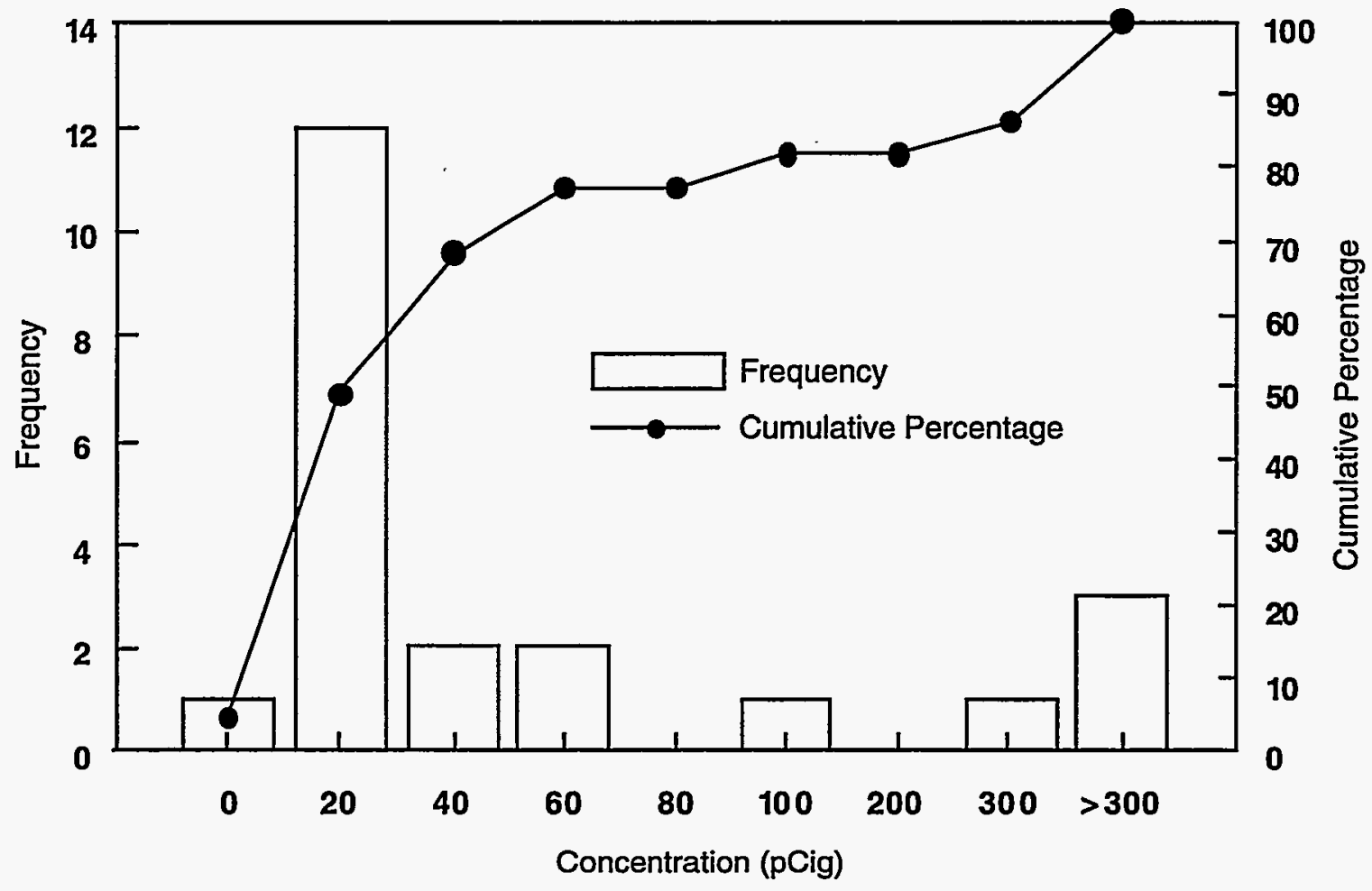

Figure 4-8. Distribution of U concentrations in YPG soil samples. 


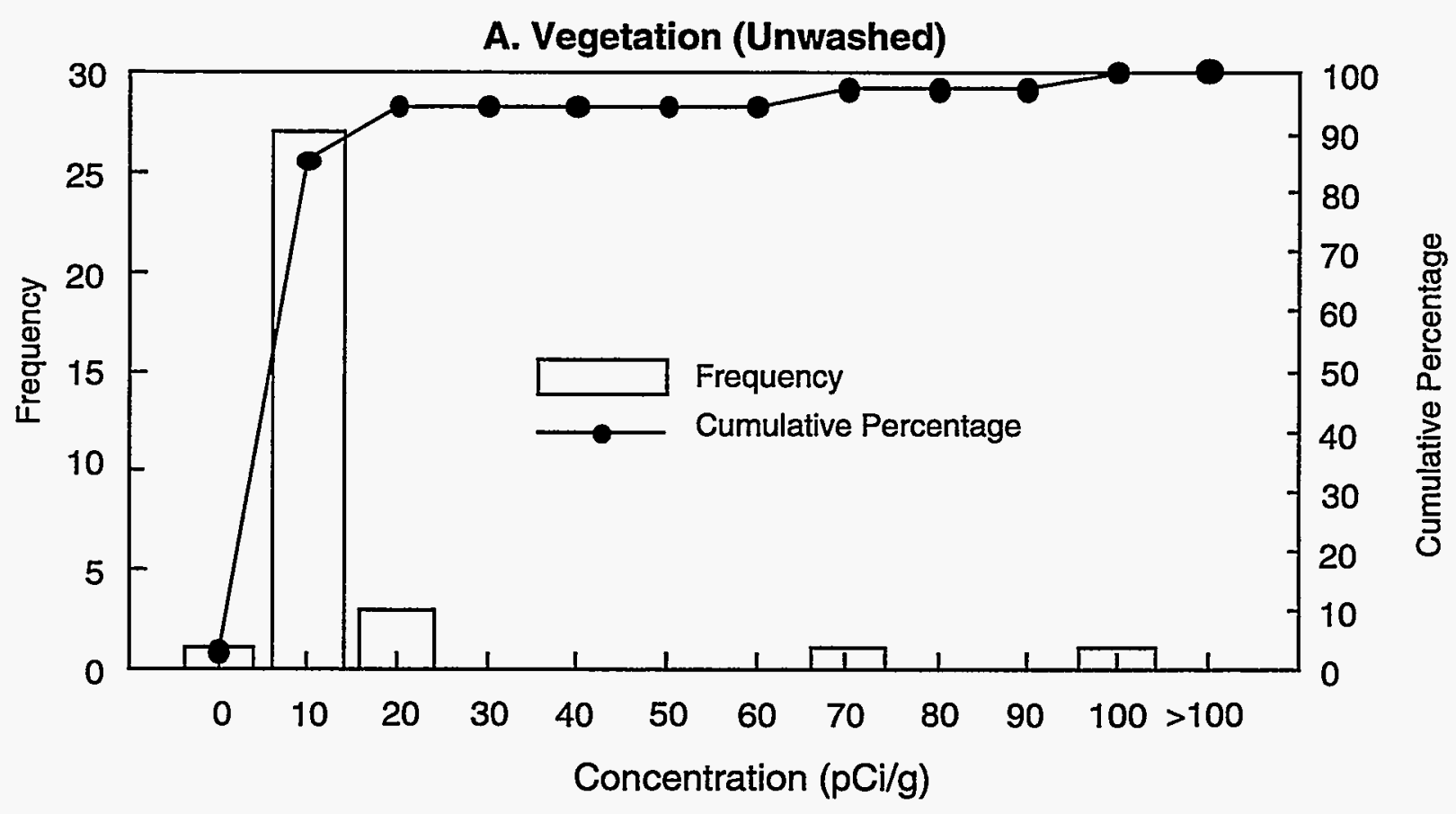

\section{B. Litter}

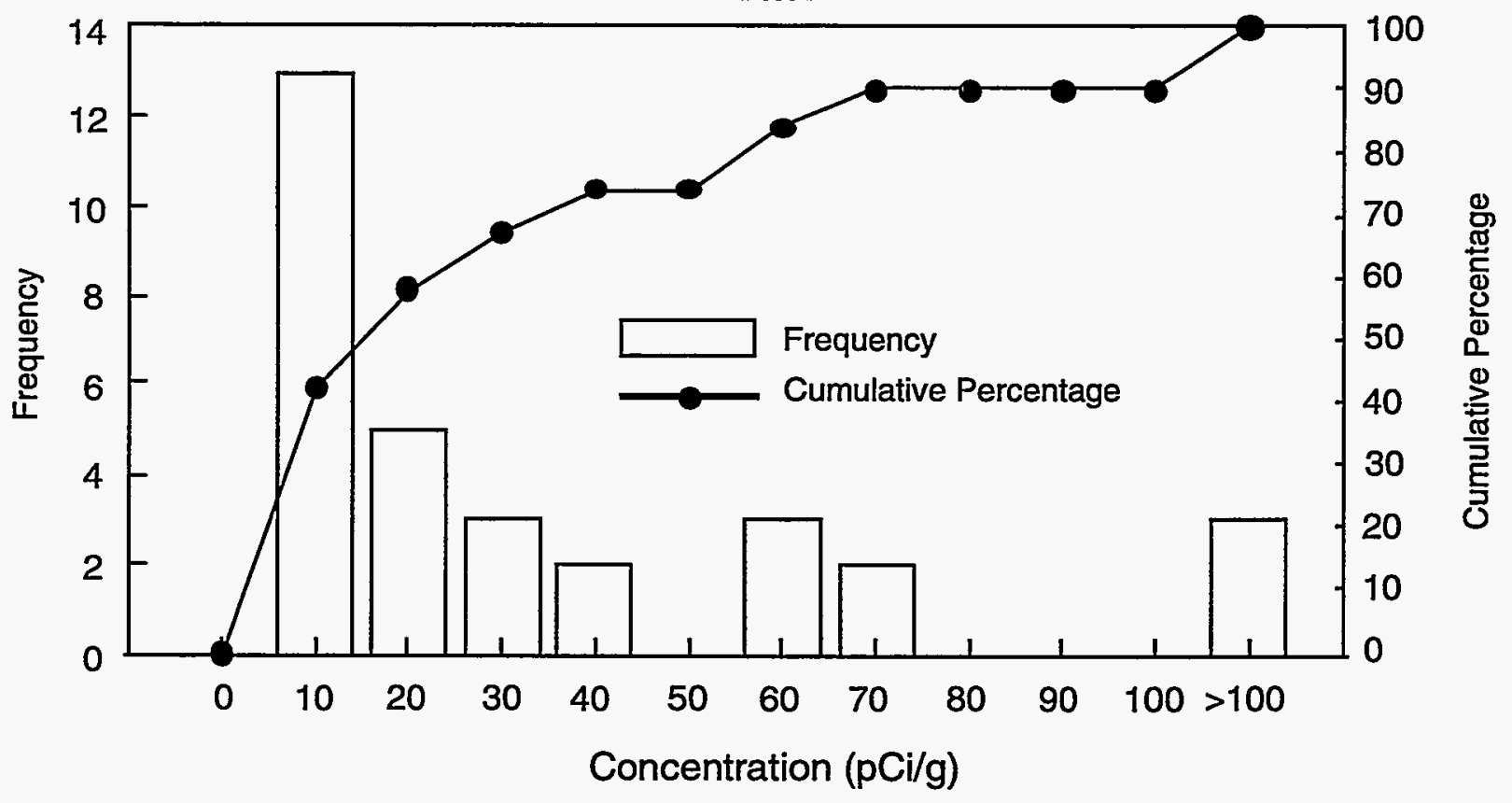

Figure 4-9. Distribution of $U$ concentrations in unwashed vegetation (A) and litter (B) collected at YPG. 


\subsubsection{Invertebrate Samples}

Invertebrates were pooled by species to ensure that enough mass was submitted for reasonable analytical results. Summary results are shown in Table 4-3 and Figure 4-10, and all other data are given in Table E-4. The $95 \%$ confidence interval for $U$ concentrations was $1.7 \times 10^{-1} \mathrm{pCi} / \mathrm{g}$ (fresh weight) to 6.7 $\mathrm{pCi} / \mathrm{g}$ (fresh weight). U concentrations in 33 samples (77\%) were less than $2 \mathrm{pCi} / \mathrm{g}$; in only 9 samples (20\%) the concentration exceeded $2 \mathrm{pCj} / \mathrm{g}$ (Figure 4-10). Isotope ratios show that 33 of the 43 samples contained DU and 2 samples contained natural $U$; the ${ }^{235} U$ concentrations of 8 samples were not reported so ratios were not calculated. The data confirm that $\mathrm{DU}$ is ingested by invertebrates or carried on the surface of the invertebrates.

\subsubsection{Small Herbivores}

4.4.3.4.1. Pocket Mice. Samples of pocket mice (Perognathus) carcasses, kidneys, and livers were collected for detailed analyses of DU uptake and so that the potential for adverse effects due to radiation and chemical toxicity could be estimated. Uranium concentrations in carcass samples averaged $1.2 \mathrm{pCi} / \mathrm{g}$ (fresh weight) and the median value was $1.1 \times 10^{-1} \mathrm{pCi} / \mathrm{g}$ (fresh weight), indicating a highly skewed distribution. Without the maximum value of $4.0 \mathrm{pCi} / \mathrm{g}$, the average concentration calculated was $2.1 \times$ $10^{-2} \mathrm{pCi} / \mathrm{g}$, and the $95 \%$ confidence interval was $1.4 \mathrm{pCi} / \mathrm{g}$ (fresh weight) to $2.9 \times 10^{-1} \mathrm{pCi} / \mathrm{g}$ (fresh weight). Thirty-nine pocket mice carcass samples were analyzed (Table E-5) and the results are summarized in Table 4-3. Thirty-six samples (approximately $85 \%$ ) had U concentrations of $0.8 \mathrm{pCi} / \mathrm{g}$ or less (fresh weight), and only six had concentrations of $1 \mathrm{pCi} / \mathrm{g}$ or greater (fresh weight) (Figure 4-11A). The 95\% confidence interval was again large, from the detection limit to $3.2 \mathrm{pCi} / \mathrm{g}$ (fresh weight) with a maximum $U$ concentration of $40 \mathrm{pCi} / \mathrm{g}$ (fresh weight). Carcass samples included pelts, thus a small particle of DU included in the pelt could explain the largest concentrations in the range. Samples were not washed before analysis because we wanted to know the total amount of DU that could be transfered to animals consuming pocket mice. Figure 4-10A shows the distribution of values for the carcass samples.

Thirty kidney samples from pocket mice were analyzed (Table E-5). Concentrations of ${ }^{238} U$ or ${ }^{235} U$ were below detection in 17 of the 30 samples, and results from the 14 samples with concentrations greater than the detection limits are summarized in Table 4-3. Twelve of the 14 samples with $U$ greater than the detection limits contained $0.1 \mathrm{pCi} / \mathrm{g}$ or less ${ }^{238} \mathrm{U}$, one sample contained $0.22 \mathrm{pCi} / \mathrm{g}$, and one sample contained $0.6 \mathrm{pCi} / \mathrm{g}$ (Figure 4-11B). Only two samples contained ${ }^{235} \mathrm{U}$ greater than detection limits, but these results yielded isotopic ratios that were too large to be credible. Kidney burdens were also calculated from the field data and are also shown in Table 4-3 and Table E-5. The mean kidney burden was $1.3 \times 10^{-1} \mu \mathrm{g} / \mathrm{g}$-kidney (fresh weight) and the upper $95 \%$ confidence interval for kidney burdens was $2.2 \times 10^{-1} \mu \mathrm{g} / \mathrm{g}$-kidney (fresh weight). The result from Sample \#153 was $0.6 \mu \mathrm{g} / \mathrm{g}$-kidney (fresh weight). The result for Sample \#153 approached the 1- $\mu \mathrm{g} / \mathrm{g}$-kidney threshold level and suggests that nephrotoxic effects could be induced in pocket mice. 


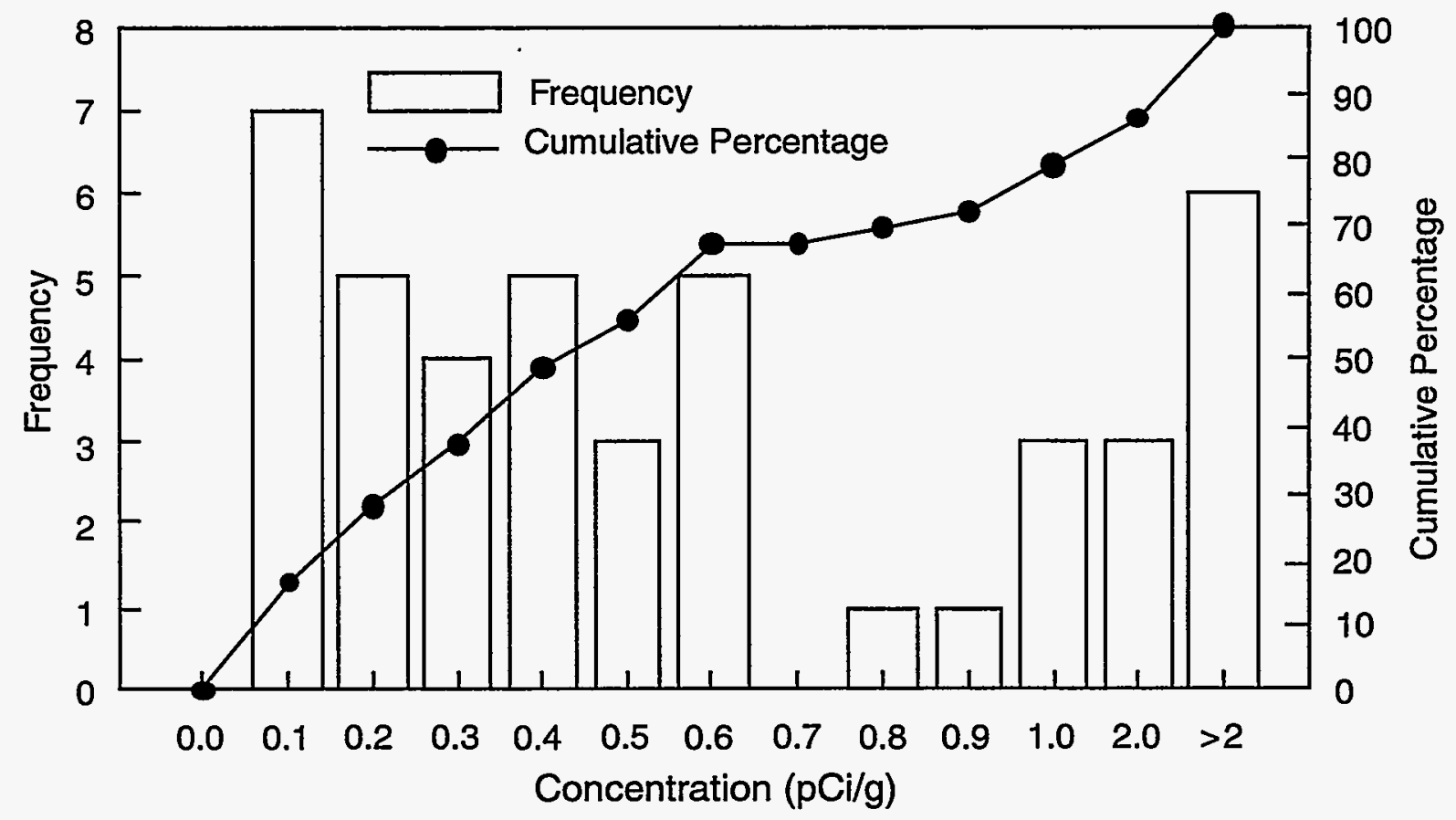

Figure 4-10. Distribution of U concentrations in invertebrates collected at YPG. Concentrations based on fresh tissue weight. 


\section{A. Perognathus Carcasses}

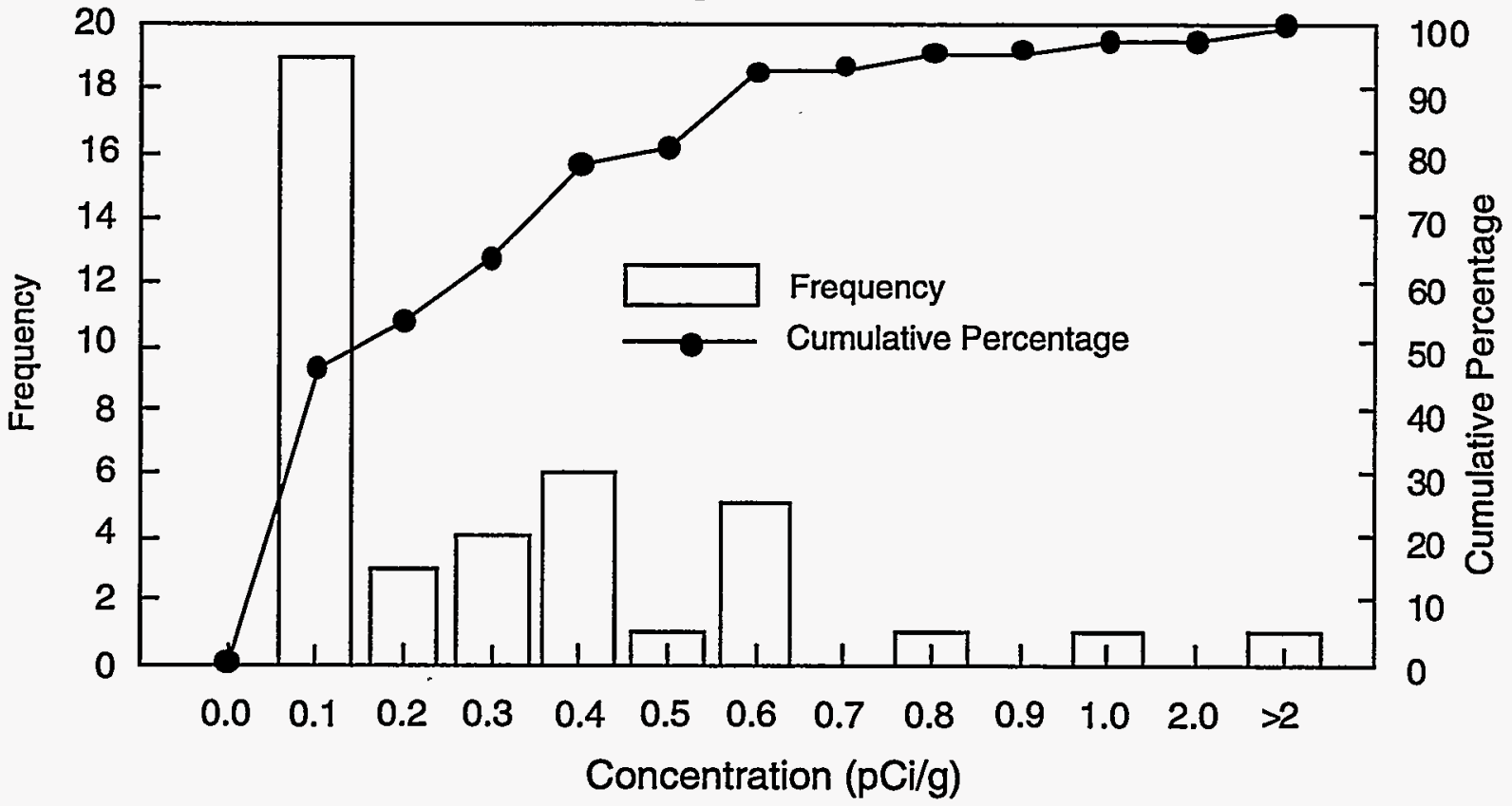

B. Perognathus Kidney

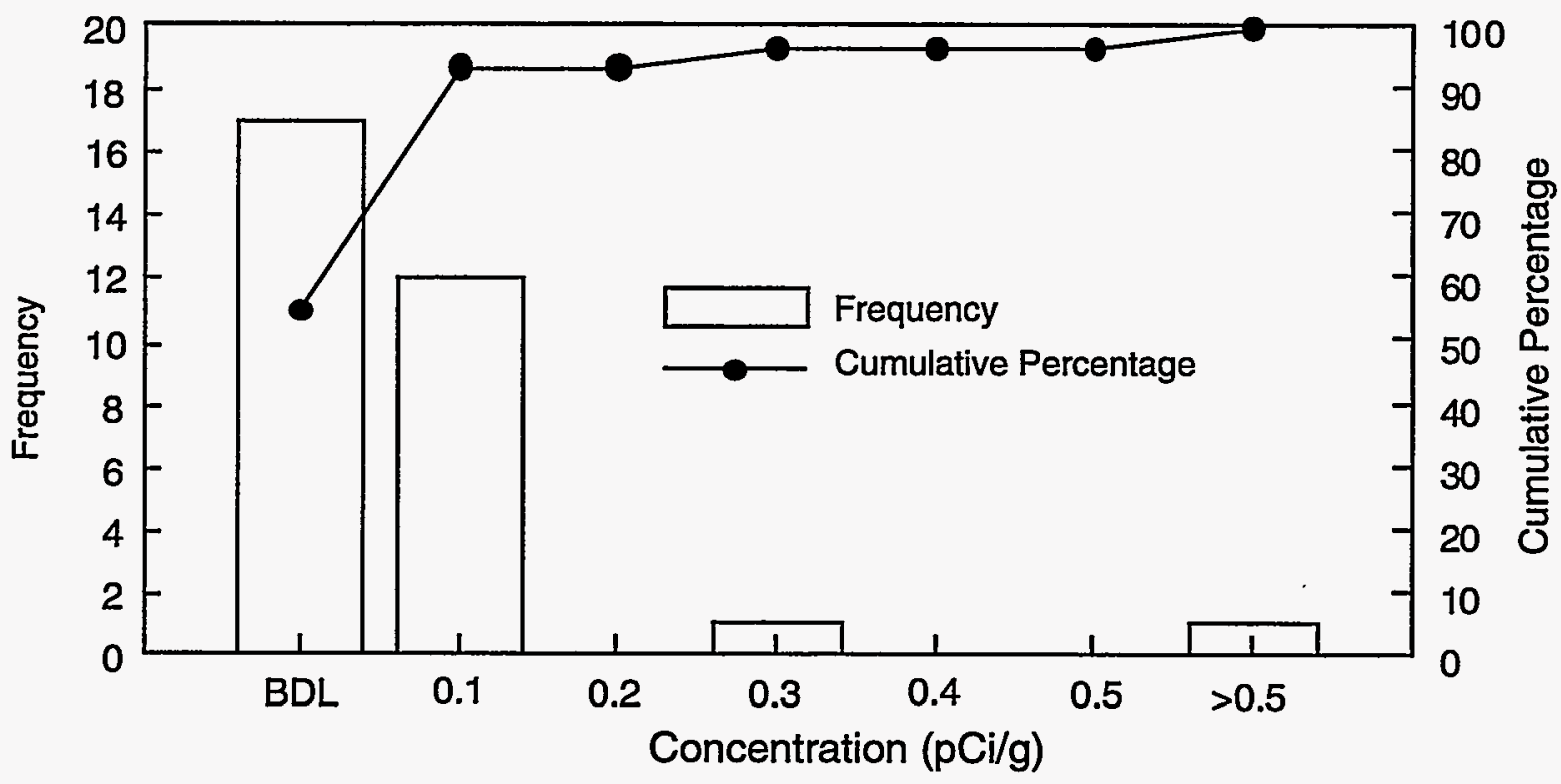

Figure 4-11. Distribution of $U$ concentrations in pocket mice carcasses (A) and kidneys (B). Concentrations based on fresh tissue weight in both plots. BDL (B) indicates that concentrations of ${ }^{238} \mathrm{U}$ and ${ }^{235} \mathrm{U}$ were below detection limits. 
Histopathology of some kidney samples supports the possibility of nephrotoxic effects, whichwill be discussed in the risk estimation section below.

4.4.3.4.2. Kangaroo Rats. Kangaroo rats (Dipodomys meriamii) were also sampled in the field, and $\mathrm{U}$ concentration data from carcasses, kidneys, and livers were obtained. The carcass concentrations averaged $1.4 \times 10^{-1} \mathrm{pCi} / \mathrm{g}$ (standard deviation of $4.1 \times 10^{-2} \mathrm{pCi} / \mathrm{g}, 8$ samples) and the $95 \%$ confidence interval was $1.9 \times 10^{-2} \mathrm{pCi} / \mathrm{g}$ (fresh weight) to $2.7 \times 10^{-1} \mathrm{pCi} / \mathrm{g}$ (fresh weight). Table 4-3 summarizes the results, and all results are shown in Table E-5.

Liver samples from kangaroo rats contained low $U$ concentrations, with the $95 \%$ confidence interval from $1.3 \times 10^{-3} \mathrm{pCi} / \mathrm{g}$ (fresh weight) to $2.7 \times 10^{-2} \mathrm{pCi} / \mathrm{g}$ (fresh weight). U concentrations in kidney samples also were low, with the $95 \%$ confidence interval from $4.2 \times 10^{-2} \mathrm{pCi} / \mathrm{g}$ (fresh weight) to $3.6 \times 10^{-1}$ $\mathrm{pCi} / \mathrm{g}$ (fresh weight). Kidney burdens calculated from the field data suggested that no toxicological effects are expected based on the threshold value of $1 \mu \mathrm{g} / \mathrm{g}$-kidney. The $95 \%$ confidence interval for kidney burdens was $1.1 \times 10^{-1} \mu \mathrm{g} / \mathrm{g}$-kidney (fresh weight) to $9.8 \times 10^{-1} \mu \mathrm{g} / \mathrm{g}$-kidney. Two samples, \#150 and \#168, had kidney burdens of $1.6 \mu \mathrm{g} / \mathrm{g}$-kidney (fresh weight) and $1.2 \mu \mathrm{g} / \mathrm{g}$-kidney (fresh weight), respectively. These results suggest that nephrotoxic effects may occur in kangaroo rats due to $U$ ingestion. The ${ }^{235} \mathrm{U}$ data gave erroneous isotopic ratios for all kangaroo rat kidney samples, and differentiating natural $U$ from DU in the kidney samples was not possible.

4.4.3.4.3. White-throated Woodrat. White-throated woodrats (Neotoma albigula) were sampled from GP 17A and GP 20. U concentrations in carcass samples from 14 individuals averaged $2.5 \mathrm{pCi} / \mathrm{g}$ (fresh weight) and had a median value of $4.9 \times 10^{-1} \mathrm{pCi} / \mathrm{g}$ (fresh weight). The $95 \%$ confidence interval of the reported concentrations was from the detection limit to $6.1 \mathrm{pCi} / \mathrm{g}$ (fresh weight). Eleven of 14 carcass samples contained DU as indicated by isotopic ratios less than 0.0050 , and three samples had ${ }^{235} \mathrm{U}$ concentrations below the detection limits. Carcass samples included pelts and muscle tissue but no internal organs (Table 4-3; Table E-5).

Liver samples from 10 individuals had $U$ concentrations greater than detection limits. The concentrations averaged $2.7 \times 10^{-3} \mathrm{pCi} / \mathrm{g}$ (fresh weight), the median value was $2.7 \times 10^{-3} \mathrm{pCi} / \mathrm{g}$ (fresh weight), and the $95 \%$ confidence interval was $1.5 \times 10^{-3} \mathrm{pCi} / \mathrm{g}$ to $3.9 \times 10^{-3} \mathrm{pCi} / \mathrm{g}$. All ${ }^{235} \mathrm{U}$ data were lower than the detection limits so no isotopic ratios were calculated (Table 4-3; Table E-5).

Kidney samples from 12 individuals contained $U$ concentrations greater than detection limits. The concentrations averaged $2.0 \times 10^{-2} \mathrm{pCi} / \mathrm{g}$ (fresh weight), the median value was $1.1 \times 10^{-2} \mathrm{pCi} / \mathrm{g}$ (fresh weight), and the $95 \%$ confidence interval was $6.1 \times 10^{-3} \mathrm{pCi} / \mathrm{g}$ to $3.4 \times 10^{-2} \mathrm{pCi} / \mathrm{g}$. Four samples contained ${ }^{235} \mathrm{U}$ concentrations greater than detection limits, but three of the isotopic ratios were unreasonably high. The isotopic ratio of one sample (\#12), however, suggested that DU was the U source. Kidney burdens averaged $4.3 \times 10^{-2} \mu \mathrm{g} / \mathrm{g}$-kidney (fresh weight), the median value was $1.7 \times 10^{-2}$ $\mu \mathrm{g} / \mathrm{g}$-kidney (fresh weight), and the $95 \%$ confidence interval ranged from $1.2 \times 10^{-2} \mu \mathrm{g} / \mathrm{g}$-kidney to $7.4 \times$ 
$10^{-2} \mu \mathrm{g} / \mathrm{g}$-kidney (Table 4-3; Appendix E). Kidney burdens suggest that no adverse nephrotoxic effects from $U$ or DU occurred in the individuals sampled.

4.4.3.4.4. Desert Woodrat. Desert woodrats (Neotoma lepida) were collected from the firing lines of GP 17A and GP 20. U concentrations from carcass samples of five individuals averaged $3.2 \times 10^{-2} \mathrm{pCi} / \mathrm{g}$ (fresh weight), the median value was $3.9 \times 10^{-2} \mathrm{pCi} / \mathrm{g}$, and the $95 \%$ confidence interval was $1.8 \times 10^{-2}$ $\mathrm{pCi} / \mathrm{g}$ to $4.6 \times 10^{-2} \mathrm{pCi} / \mathrm{g}$ (Table 4-3; Table E-5). Two carcass samples contained ${ }^{235} \mathrm{U}$ concentrations greater than the detection limit. One of the two samples indicated DU, but the other isotopic ratio was unreasonably large. Carcass samples included pelt and muscle but no internal organs.

Two of five samples of livers from desert woodrats contained $U$ concentrations greater than the detection limit. The reported concentrations ranged from $8.5 \times 10^{4} \mathrm{pCi} / \mathrm{g}$ (fresh weight) to $1.4 \times 10^{-3}$ $\mathrm{pCi} / \mathrm{g}$ (fresh weight). ${ }^{235} \mathrm{U}$ concentrations in both samples were lower than the detection limit so no isotopic ratios were calculated. Two kidney samples also contained $U$ concentrations greater than the detection limits, and both samples contained $2.5 \times 10^{-3} \mathrm{pCi} / \mathrm{g}$ (fresh weight). Neither sample contained a

${ }^{235} \mathrm{U}$ concentration greater than the detection limit, and no isotopic ratios were calculated. Kidney burdens calculated from the data were $6.8 \times 10^{-3} \mu \mathrm{g} / \mathrm{g}$-kidney (fresh weight) and suggest that no nephrotoxic effects occurred in these individuals (Table 4-3; Table E-5).

4.4.3.4.5. Desert Iguanas. Desert igaunas (Dipsosaurus dorsalis) collected from GP 17A and GP 20 provided samples of carcasses and livers. Four carcass samples contained $U$ concentrations greater than detection limits and averaged $2.2 \times 10^{-1} \mathrm{pCi} / \mathrm{g}$ (fresh weight) with a $95 \%$ confidence interval of $9.3 \times 10^{-2}$ $\mathrm{pCi} / \mathrm{g}$ (frsh weight) to $3.5 \times 10^{-1} \mathrm{pCi} / \mathrm{g}$ (fresh weight). All of the carcass samples contained sufficient ${ }^{235} \mathrm{U}$ for detection, and three of the four samples showed $D U$ as the source of $U$ by isotopic ratios greater than 0.0050. The isotopic ratio calculated for the fourth sample was unreasonably high. Two liver samples from desert iguanas were analyzed, and the results ranged from $2.6 \times 10^{-3} \mathrm{pCi} / \mathrm{g}$ (fresh weight) to $6 \times 10^{-1}$ $\mathrm{pCi} / \mathrm{g}$ (fresh weight). The ${ }^{235} \mathrm{U}$ concentration was greater than the detection limit in the sample with the largest concentration, and the isotopic ratio of 0.0023 indicated that DU was the source of the $U$.

\subsubsection{Large Herbivores}

Large herbivores are represented in our samples by desert cottontails (Sylvilagus audoboni) and black-tail jack rabbits (Lepus californicus). Individuals were collected near the firing lines of GP 17A and GP 20.

4.4.3.5.1. Desert Cottontails. Five individuals were collected, and carcass and muscle samples were dissected, analyzed, and combined in the data analysis (Table 4-3; Table E-6). Seven of ten samples contained detectable U. Samples $297 \mathrm{CA}$ and $300 \mathrm{MA}$, both from GP 20, showed DU, whereas the ${ }^{235} \mathrm{U}$ concentration of all other carcass and muscle samples were below detection limits and no isotopic ratios were calculated. A replicate of Sample $300 \mathrm{CA}$ (\# $300 \mathrm{MB}$ ) contained no detectable ${ }^{238} \mathrm{U}$ or ${ }^{235} \mathrm{U}$, suggesting that DU particulates were associated with Sample $300 \mathrm{MA}$ and were responsible for the high 
concentration. The average detectable $U$ concentration was $9.7 \mathrm{pCj} / \mathrm{g}$ (fresh weight) with a median of 6.9 $\times 10^{-4} \mathrm{pCi} / \mathrm{g}$ (fresh weight) when all results were considered. The average detectable $U$ concentration decreased to $7 \times 10^{-3} \mathrm{pCi} / \mathrm{g}$ (fresh weight) when Sample $300 \mathrm{MA}$ was omitted, and the median decreased slightly to $5.3 \times 10^{-4} \mathrm{pCi} / \mathrm{g}$ (fresh weight). The $95 \%$ confidence interval for carcass and muscle samples without Sample $300 \mathrm{MA}$ was from the detection limit to $1.6 \times 10^{-2} \mathrm{pCi} / \mathrm{g}$ (fresh weight). Carcass and muscle results show the importance of DU particulates in pelts and/or on tissue samples.

Results of liver samples from the same individuals used for carcass and muscle samples showed only one value greater than the detection limit. Sample 298 was taken from the same individual that provided Sample $297 \mathrm{CA}$ and was collected from the GP 20 firing line. The liver concentration was $1.1 \times 10^{-3}$ $\mathrm{pCi} / \mathrm{g}$ (fresh weight), though the ${ }^{235} \mathrm{U}$ concentration was lower than the detection limit so no isotopic ratio could be calculated.

Results of kidney samples showed two of five samples with $U$ concentrations greater than the detection limit. Sample 299 was taken from the same individual that provided Samples 297 and 298. Sample 223 was taken from an individual collected at GP 17A and the same individual that supplied Samples $221 \mathrm{CA}$ and 222. U concentrations were $3.8 \times 10^{-3} \mathrm{pCi} / \mathrm{g}$ (fresh weight; Sample 299) and $1.6 \times$ $10^{-2} \mathrm{pCi} / \mathrm{g}$ (fresh weight; Sample 223). Kidney burdens were $1 \times 10^{-2} \mu \mathrm{g} / \mathrm{g}$-kidney (fresh weight) for Sample 299 and $4.4 \times 10^{-2} \mu \mathrm{g} / \mathrm{g}$-kidney (fresh weight) for Sample 223. Neither kidney burden was expected to cause nephrotoxic damage since both were less than the suggested threshold of $1 \mu \mathrm{g} / \mathrm{g}$ kidney. ${ }^{235} \mathrm{U}$ was not detected in any of the kidney samples so no isotopic ratios were calculated.

4.4.3.5.2. Black-tailed Jack Rabbit. Carcass and muscle data were combined for this analysis, and results showed that eight of thirteen samples contained $U$ greater than the detection limits (Table 4-3 and Table E-6). Results of ${ }^{235} \mathrm{U}$ for one sample, Sample $233 \mathrm{CA}$, were greater than the detection limit, but the corresponding isotopic ratio was meaningless due to analytical error. The average detectable $\mathrm{U}$ concentration was $5.3 \times 10^{-4} \mathrm{pCi} / \mathrm{g}$ (fresh weight) and the largest concentration was $1.3 \times 10^{-3} \mathrm{pCi} / \mathrm{g}$ (fresh weight) in Sample 229 CB, collected from GP 20. A replicate of Sample 229 CB (Sample 229 A) provided results that were in excellent agreement with those from Sample 229 CB (Table E-6).

Only a single liver sample, Sample 230 , contained a U concentration greater than the detection limit. This sample was taken from the same individual that provided Sample 229. ${ }^{235} \mathrm{U}$ was not detected in any of the liver samples, so no isotopic ratios were calculated.

Four of the five kidney samples analyzed contained $U$ concentrations greater than the detection limits (Table 4-3 and Table E-6). Sample 231 was taken from the same individual that provided Samples 229 and 230. Sample 303 was also taken from an individual collected on the GP 20 firing line, whereas Samples 235 and 239 were taken from individuals collected on the GP 17A firing line. The average detectable $U$ concentration was $3.2 \times 10^{-4} \mathrm{pCi} / \mathrm{g}$ (fresh weight), and the largest concentration was $7.1 \times 10^{-}$ ${ }^{4} \mathrm{pCi} / \mathrm{g}$ (fresh weight). ${ }^{235} \mathrm{U}$ concentrations were below detection limits for all kidney samples so no isotopic ratios were calculated. The average kidney burden was $8.6 \times 10^{-4} \mu \mathrm{g} / \mathrm{g}$-kidney (fresh weight) and 
the largest was $1.9 \times 10^{-3} \mu \mathrm{g} / \mathrm{g}$-kidney (fresh weight). The results suggest no nephrotoxic effects due to $\mathrm{U}$ ingestion.

\subsubsection{Insectivores (Lizards)}

Insectivores are represented in our samples by a variety of lizards. Whole lizards were analyzed with no attempt to remove internal organs or to wash them before preparation. The lizard samples were prepared the same as all other biotic samples except for dissection. The descriptive statistics are shown in Table 4-3, and results for each sample are shown in Table E-7. The mean U concentration for the entire compartment was $4.0 \mathrm{pCi} / \mathrm{g}$ (Fresh weight) with a standard deviation of $15 \mathrm{pCi} / \mathrm{g}$ (fresh weight). The median value was $1.0 \times 10^{-1} \mathrm{pCi} / \mathrm{g}$ (fresh weight), considerably less than the mean. The distribution of the values shows that 31 of 35 samples (89\%) are lower than the mean with four samples well above the mean (Figure 4-12). The bimodal distribution suggests that little $U$ is ingested and retained by lizards and that the primary mechanism for $U$ transport by lizards could be attachment to body surfaces. It is possible that the four samples with the highest concentrations included discrete particles of DU on skin surfaces and thus resulted in the high concentrations. However, this conclusion is uncertain without data from washed and unwashed samples.

Isotopic ratios less than 0.0050 indicated that 17 of 35 samples contained DU; 2 contained natural $U$, 1 may have contained DU and natural U, 3 had ratios too large to be meaningful, and 12 were not calculated because ${ }^{235} \mathrm{U}$ concentrations were below detection limits. Concentrations from samples with isotopic ratios that indicate the presence DU ranged from about $1.3 \times 10^{-1} \mathrm{pCi} / \mathrm{g}$ to $65 \mathrm{pCi} / \mathrm{g}$. Natural U is indicated in samples with concentrations from about $1 \times 10^{-1} \mathrm{pCi} / \mathrm{g}$ to about $4 \times 10^{-1} \mathrm{pCi} / \mathrm{g}$, but not in samples with concentrations greater than $1 \mathrm{pCi} / \mathrm{g}$. Samples less than about $1 \times 10^{-1} \mathrm{pCi} / \mathrm{g}$ did not contain sufficient ${ }^{235} \mathrm{U}$ for a reliable ratio to be calculated.

\subsubsection{Predators}

Predators are represented only by two sidewinders (Crotalus cerastes) and one sample of coyote (Canis latrans) scat. The $U$ concentration of the scat sample was below detection. The average $U$ concentration in the two sidewinders was $1.5 \mathrm{pCi} / \mathrm{g}(2.88$ and $0.21 \mathrm{pCi} / \mathrm{g})$. The small sample size for this compartment limits the conclusions that can be made. The range of the two reported values does not support or refute uptake of $U$ or DU by sidewinders preying on other animals of the impact areas. Both samples, however, indicate that the $U$ is from DU as the isotopic ratios were 0.0021 and 0.0033 . 


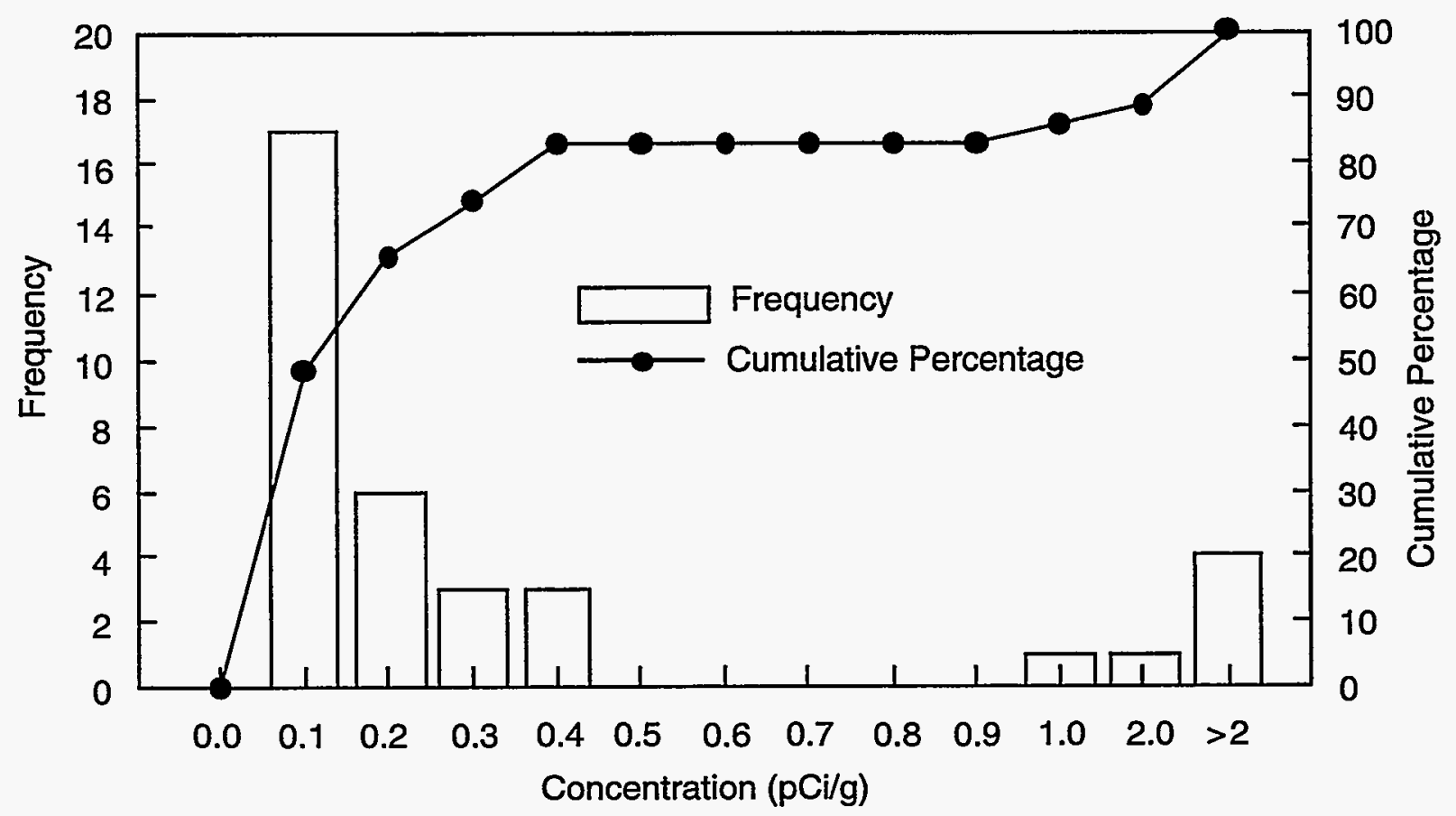

Figure 4-12. Distribution of U concentrations in lizard samples. Concentrations based on fresh tissue weight in both plots. 


\subsubsection{Discussion}

The samples collected from YPG represent all of the compartments in the conceptual model shown in Figure 4-5. With the exception of the predators compartment, each compartment was represented by at least 10 samples. Distributions of the concentrations in the invertebrates, vegetation, small herbivores, soil, and litter were computed for use in the risk estimations. Estimates of the uncertainty or variability in each of the compartments were obtained from the data reported and reduced the uncertainty in the risk estimates. Risk estimates will be presented in the next chapter.

The data indicate that DU accumulates on vegetation surfaces from resuspended soil or possibly soil relocated from rainsplash (Dreicer et al. 1984; Anspaugh et al. 1975). Ingestion of soil-containing DU is likely because soil is ingested during consumption of vegetation or predation and through nondietary behavior such as pelt preening. $U$ data from internal organ samples suggest that $U$ and possibly $D U$ are ingested through food intake and nondietary pathways. Field data also indicate that pelts or body surfaces are important in DU transport through the food chain. Mammals and reptiles that consume insects, reptiles, or other mammals will most likely consume DU carried in pelts and on body surfaces. Samples of carcasses that included pelts from pocket mice, kangaroo rats, white-throated woodrats, desert woodrats, and cottontails contained U from DU. Samples of insects and lizards, either whole animals or carcasses, also contained $U$ from DU that would be ingested by other animals consuming insects or reptiles.

Field data from YPG also show that detectable $U$ was measured in internal organs from different compartments. Kidney burdens in two kangaroo rat samples exceeded the $1-\mu \mathrm{g} / \mathrm{g}$-kidney (fresh weight) suggested toxicity threshold, and one pocket mouse kidney approached the suggested threshold. These results suggest nephrotoxic effects are possible in pocket mice and kangaroo rats, as well as in other species of small herbivores. The field data also indicate that there is no obvious trend that DU is systematically or hierarchically concentrated in the food web, that is, that DU does not bioaccumulate at YPG. 


\section{DOSE CALCULATIONS AND ESTIMATES OF HEALTH EFFECTS}

\subsection{Dose Calculations for Human Health Effects}

\subsubsection{Methodology}

We estimated the radiological and toxicological doses from DU to humans at APG and YPG by analysis of different exposure pathways. The pathways considered for human health endpoints at APG were ingestion of meat from deer hunting, consumption of fish taken from local streams and rivers, consumption of contaminated drinking water, inhalation of DU-containing dust, and exposure to the skin surface from DU in the surface soils. A hypothetical hunting scenario was developed for YPG in order to estimate potential human health effects of consuming rabbits from the GP 20 and GP 17A impact areas. Pathways for ecological endpoints were discussed in Chapters 3 and 4.

Potential transfer of DU through the environment to animals, vegetation, and humans was shown previously for APG (Figure 3-4) and for YPG (Figure 4-5). Transfer to humans was modeled by adding human consumption of deer meat to the steady-state model for deer and by summing the $U$ or DU concentrations from all sources of food and water obtained from APG. The approach was modified from that suggested by the NCRP (NCRP 1984a). All beef in the NCRP model diet was replaced with deer tissue from hunted deer. Likewise, all fish in the NCRP model diet was replaced with fish taken from APG. Drinking water was also considered contaminated, although there was little field data to support this assumption. It is unrealistic to assume that the aquifers within which the monitoring wells are located would produce water of high enough quality to meet drinking water standards. We wanted to include possible effects of consuming contaminated drinking water, however, so this pathway was added to the dose assessments. The exposure scenarios modeled are based on the field data collected from APG and YPG, and include conservative yet realistic estimates of some parameters. We intentionally avoided "worst case" assumptions unless absolutely necessary because they are unrealistic in most instances.

We evaluated the data available for each parameter in the model. Since most of the uncertainty in the predictions comes from uncertainty in the parameter estimates, we included the uncertainty data in the dose calculations. This was done by describing each parameter as a probability distribution of values instead of point estimates. Thus, instead of a single value for each parameter, such as $U$ concentrations in soils, distributions of values were used for the model. Uncertainty analysis is discussed in Section 3.6.2 in more detail.

Estimating probability distributions was difficult in some cases because only sparse site-specific data were available or the data represented a wide range of concentrations without discernible trends. Several parameters used in the models were estimates; all parameters with estimated values were described by uniform distributions. Use of probability distributions for parameter values allowed us to test the uncertainty and sensitivity of the models with Monte Carlo methods and also allowed preservation of the variability in the values we used. Appendix F shows the parameters and the probability distributions used in the predictions of DU transport to humans.

Toxicological data vary on adverse effects to humans from ingestion of DU. The literature supported a kidney burden of $1 \mu \mathrm{g} / \mathrm{g}$-kidney as a possible threshold concentration, and after a safety factor of 10 is 
applied, the suggested threshold limit for human exposure is $0.1 \mu \mathrm{g} / \mathrm{g}$ (Kocher 1989; Leggett 1989; Zhao and Zhao 1990). Kidney burdens were estimated from the results of the predictions of radiological doses to kidneys by converting the activity concentration $(\mathrm{pCi} / \mathrm{g})$ to mass concentration $(\mu \mathrm{g} / \mathrm{g}) \mathrm{using}$ the specific activities of $0.72 \mathrm{pCi} / \mu \mathrm{g}$ for natural $U$ and $0.37 \mathrm{pCi} / \mu \mathrm{g}$ for DU. The kidney burdens of $U$ from APG deer were also measured and compared with $U$ burdens in kidneys from deer that were not exposed to the APG impact area soils and vegetation. These data are discussed in Chapter 3.

Potential adverse effects to humans were also projected 100 years from the present at APG and YPG using the RESRAD code (Yu et al. 1993). Immediate effects of DU in soils, water, and the food chain are minimal, as shown later in this chapter. However, the slow transport of DU through soils to groundwater and surface water at APG and by erosion at YPG suggest that adverse effects could occur in the future. RESRAD is an available tool for such projections and was compatible with the field data we collected at both APG and YPG.

\subsubsection{Assumptions}

The exposure models used field and laboratory data extensively for estimating radiological and toxicological doses to humans and ecosystems (ICRP 1987; NCRP 1984b). Several assumptions were still required in order to perform the necessary dose calculations for different organs of interest.

The diet of adult humans was altered by substituting deer tissue for all beef consumed yearly. In specific cases this was not a bad assumption, but it represents an unrealistic scenario for members of the general public that do not consume deer tissue. Informal surveys of deer hunters indicated that there would not be enough deer tissue supplied from hunting to allow $100 \%$ substitution every year. The assumption that all fish and shellfish are taken from the APG area is reasonable in light of the rich history of aquatic resource use in the Chesapeake Bay area. The consumption rate for fish and shellfish may be considerably higher for fishermen than the rates we used since this freshwater resource is so easily utilized by recreational and commercial fishermen.

Water concentrations were measured as part of the field sampling campaign, and few data indicated that $U$ or DU was detectable. Environmental monitoring data also indicate low to undetectable amounts of $U$ and DU in the surface water and groundwater at APG. Using drinking water values of $1-3 \mathrm{pCi} / \mathrm{L}$ is a conservative assumption that may drive the radiation doses higher than would actually be reported at APG. The median $U$ concentration in the water supplies used for drinking in the United States ranges from 0.2 to $0.7 \mathrm{pCi} / \mathrm{L}$ with values from 0.01 to $1000 \mathrm{pCi} / \mathrm{L}$ reported (Wrenn et al. 1985; Cothern and Lappenbusch 1983). We chose higher values than the average and significantly higher values than we measured to allow for variation in actual $U$ water concentrations and possible effects on humans. While downward revision of the values used for predictions would reduce the predicted radiological and toxicological doses to humans, and therefore, reduce the risk of death or occurrence of cancers, the doses are already immeasurably low. Therefore, revision of water concentrations was not done.

Dose conversion factors (DCFs) were used to convert ingested concentrations to radiological doses. Usually two DCFs were listed, and we used the higher DCF in our calculations. As with the water 
concentrations, calculations using the lower DCF values reduced an already immeasurably small dose to humans so the lower DCF values were not used.

The assumptions used for the prediction of radiological doses and cancer detriments at APG are based on a recreational hunting or occasional use scenario. Site users are assumed to occupy the site for two one-week periods each year. During that time they sleep on the ground, eat food and consume water brought in from off-site, and hunt and fish the impact area and streams that drain the impact area. This scenario represents the current hunting program at APG with the addition of more fishing and camping in the impact area. A residential scenario for the impact area was not used because of the unrealistic assumptions such as farming on the impact area and the use of groundwater as drinking water.

A hypothetical scenario was developed for YPG. There is no hunting program currently in effect at YPG, but there is a potential for human consumption of rabbits, deer, and fowl that use contaminated areas and are hunted off-site. We modified this scenario so that rabbits from the impact area were hunted and consumed. Rabbits replace $20 \%$ of the meat in the hunters' diets for the dose estimates presented below. Also, rabbits were assumed to spend their entire lives within the impact area at YPG, obtaining all food and water from within the contaminated area. This is a fictitious scenario but allows estimation of the largest realistic dose that a hunter or poacher would be subjected to in consuming game from YPG.

\subsubsection{Results}

\subsubsection{APG Hunting Scenario}

Overall, the predicted radiological doses to humans were low. While values for yearly doses and possible cancer occurrence rates could be calculated, the magnitude of the results was too small to measure in terms of actual effects that could be attributed unambiguously to DU exposure. Table 5-1 shows the expected effects of human consumption of deer tissue in place of beef tissue predicted in the simulations. Doses to human kidney, bone, and gastrointestinal tract and the cumulative effective dose equivalent (CEDE) were calculated, and the $95 \%$ confidence intervals were estimated from the distribution of calculated doses. The range in predicted doses indicates that little if any adverse effect is expected from the consumption of deer tissue in place of beef. Table 5-2 shows the doses to humans calculated from the APG deer data presented in Chapter 3 assuming the same consumption rate. Doses calculated from field data are not significantly different at the $95 \%$ confidence level than the doses estimated from the output of the steady-state model. Moreover, the doses predicted with the model are slightly greater than the doses calculated from the field data due to uncertainty in the model parameters. The results shown in Tables 5-1 and 5-2 demonstrate that the steady-state model is a good predictor of the doses calculated from the field data on deer tissue. Tables 5-1 and 5-2 also show the small overestimation of the predictions made with the steady-state model. Additional refinements of the steadystate model were not attempted because the doses to humans were low enough that further decreases in predicted doses due to model refinements would not significantly change them.

Similarly low doses to humans were predicted from the consumption of fish (Table 5-3) and shellfish (Table 5-4). Doses to the same human tissues and the CEDE all indicate few if any adverse health effects 
from consuming these resources. Radiological doses to humans from all sources considered here (deer tissue, fish, shellfish, drinking water) are given in Table 5-5. While the doses to humans summed from all pathways is expectedly higher than from any single pathway, the summed doses are still immeasurably small. Thus, the risk of contracting cancer or dying from cancer caused by exposure to DU is minimal.

Predicted radiological doses indicate that water consumption is the largest contributor to the amount of DU ingested by humans, followed by fish, deer, shellfish, and soil ingestion, in decreasing order of significance (Table 5-5). These results were expected because of the variation in water concentrations of DU and because of the land-use scenarios chosen. Modifications of the land-use scenarios that decrease the amount of time on-site would reduce exposures, and thus doses, by reducing the amount of time humans spend in the impact area and reducing consumption of resources procured from within the impact area.

Table 5-6 shows the expected cancer detriments based on the predicted doses to humans via all pathways. Detriments are considered fatal cancers, nonfatal cancers, and severe hereditary effects (ICRP 1990). The added cancer detriments per year are infinitesimal. The largest estimate is one cancer detriment per 100 million people, an increase over "background" cancer detriment rates that is too small to measure and assign unambiguously to exposure to DU. Thus, our calculations show there is little expectation of adverse health effects due to DU exposure.

\subsubsection{RESRAD Simulations at APG}

Several additional assumptions were required in order to use RESRAD for projections of future radiological doses. The size of the impact area that constitutes the affected area was originally considered as the "tear drop" shaped portion that begins at the catch box on the Main Front Firing Range. This area covers about 1500 acres of the impact area. Recent radiological surveys at Jefferson Proving Ground (JPG) in Indiana show that the affected area is smaller than expected because lateral dispersal of penetrators was significantly less than anticipated (SEG 1995). Soil data from transects perpendicular to the firing line at APG suggest that dispersion of DU fragments is also lower than expected (Figure 3-9) and the actual affected area is smaller than 1500 acres. The affected area was estimated to be about 820 acres $\left(3.4 \times 10^{6} \mathrm{~m}^{2}\right)$ and was based on soil data from APG, trajectory modeling conducted by the Combat Systems Test Activity (CSTA), and radiological survey results from JPG. 
Table 5-1. Radiological doses to humans from consumption of deer tissue calculated from the steadystate model. Consumption rate assumes that humans replace all dietary meat with deer tissue and consume approximately $95 \mathrm{~kg}$ of meat per year. Mean values calculated from the model are shown with the $95 \%$ confidence intervals.

\begin{tabular}{|c|c|c|}
\hline $\begin{array}{c}\text { Dose to Human } \\
\text { Tissue }\end{array}$ & $\begin{array}{c}\text { Mean } \\
\text { (mrem/yr) }\end{array}$ & $\begin{array}{c}95 \% \text { Confidence Interval } \\
\text { (mrem/yr) }\end{array}$ \\
\hline Kidney & $5.7 \times 10^{-4}$ & $7.2 \times 10^{-6}$ to $4.2 \times 10^{-3}$ \\
\hline Bone & $5.7 \times 10^{-6}$ & $7.1 \times 10^{-8}$ to $4.2 \times 10^{-5}$ \\
\hline GI Tract & $2.8 \times 10^{-5}$ & $3.5 \times 10^{-6}$ to $2.1 \times 10^{-3}$ \\
\hline CEDE & $3.8 \times 10^{-7}$ & $4.9 \times 10^{-9}$ to $2.8 \times 10^{-6}$ \\
\hline
\end{tabular}

Table 5-2. Radiological doses to humans from consumption of deer tissue calculated from deer field data. Consumption rate assumes that humans replace all dietary meat with deer tissue and consume approximately $95 \mathrm{~kg}$ of meat per year. Mean values and $95 \%$ confidence intervals are shown.

\begin{tabular}{|c|c|c|}
\hline $\begin{array}{c}\text { Dose to Human } \\
\text { Tissue }\end{array}$ & $\begin{array}{c}\text { Mean } \\
\text { (mrem/yr) }\end{array}$ & $\begin{array}{c}\text { 95\% Confidence Interval } \\
\text { (mrem/yr) }\end{array}$ \\
\hline Kidney & $3.7 \times 10^{-5}$ & $8.7 \times 10^{-6}$ to $1.1 \times 10^{-4}$ \\
\hline Bone & $1.8 \times 10^{-5}$ & $4.3 \times 10^{-6}$ to $5.4 \times 10^{-5}$ \\
\hline GI Tract & $1.24 \times 10^{-6}$ & $2.9 \times 10^{-7}$ to $3.6 \times 10^{-6}$ \\
\hline CEDE & $1.1 \times 10^{-4}$ & $2.7 \times 10^{-5}$ to $3.3 \times 10^{-4}$ \\
\hline
\end{tabular}


Table 5-3. Radiological doses to humans from consumption of sunfish and perch tissue. Consumption rate assumes that sunfish and perch are the sole sources of fish in the diet and that all the fish came from impact-area streams.

\begin{tabular}{|c|c|c|}
\hline Dose to Human Tissue & $\begin{array}{c}\text { Mean } \\
\text { (mrem/yr) }\end{array}$ & $\begin{array}{c}\text { 95\% Confidence Interval } \\
\text { (mrem/yr) }\end{array}$ \\
\hline Sunfish & $3.2 \times 10^{-5}$ & $5.0 \times 10^{-6}$ to $1.1 \times 10^{-4}$ \\
\hline Kidney & $1.6 \times 10^{-5}$ & $2.5 \times 10^{-6}$ to $5.5 \times 10^{-5}$ \\
Bone & $1.1 \times 10^{-6}$ & $1.7 \times 10^{-7}$ to $3.7 \times 10^{-6}$ \\
GI Tract & $9.9 \times 10^{-5}$ & $1.6 \times 10^{-5}$ to $3.4 \times 10^{-4}$ \\
CEDE & & \\
\hline Perch & $2.9 \times 10^{-5}$ & $5.9 \times 10^{-6}$ to $8.9 \times 10^{-5}$ \\
\hline Kidney & $1.4 \times 10^{-5}$ & $2.9 \times 10^{-6}$ to $4.4 \times 10^{-5}$ \\
Bone & $9.7 \times 10^{-7}$ & $2.0 \times 10^{-7}$ to $3.0 \times 10^{-6}$ \\
GI Tract & $8.9 \times 10^{-5}$ & $1.8 \times 10^{-5}$ to $2.7 \times 10^{-4}$ \\
CEDE &
\end{tabular}

Table 5-4. Radiological doses to humans from consumption of shellfish (blue crab). Consumption rates range from 1 to $15 \mathrm{~kg}$ of crab tissue per year in the simulations.

\begin{tabular}{|c|c|c|}
\hline Dose to Human Tissue & $\begin{array}{c}\text { Mean } \\
\text { (mrem/yr) }\end{array}$ & $\begin{array}{c}95 \% \text { Confidence Interval } \\
\text { (mrem/yr) }\end{array}$ \\
\hline Kidney & $2.9 \times 10^{-5}$ & $1.6 \times 10^{-6}$ to $7.9 \times 10^{-5}$ \\
Bone & $1.4 \times 10^{-5}$ & $8.1 \times 10^{-7}$ to $3.9 \times 10^{-5}$ \\
GI Tract & $9.8 \times 10^{-7}$ & $5.5 \times 10^{-8}$ to $2.6 \times 10^{-6}$ \\
CEDE & $9.0 \times 10^{-5}$ & 0 to $2.8 \times 10^{-4}$ \\
\hline
\end{tabular}


Table 5-5. Radiological doses to humans from all sources (deer tissue, fish, shellfish, drinking water, and soil ingestion).

\begin{tabular}{|c|c|c|}
\hline Dose to Human Tissue & $\begin{array}{c}\text { Mean } \\
\text { (mrem/yr) }\end{array}$ & $\begin{array}{c}\text { 95\% Confidence Interval } \\
\text { (mrem/yr) }\end{array}$ \\
\hline Kidney & $5.6 \times 10^{-4}$ & $1.4 \times 10^{-4}$ to $1.1 \times 10^{-3}$ \\
Bone & $2.8 \times 10^{-4}$ & $6.5 \times 10^{-5}$ to $5.3 \times 10^{-4}$ \\
GI Tract & $1.9 \times 10^{-5}$ & $4.8 \times 10^{-6}$ to $3.6 \times 10^{-5}$ \\
CEDE & $1.7 \times 10^{-3}$ & $4.3 \times 10^{-4}$ to $3.3 \times 10^{-3}$ \\
\hline
\end{tabular}

Table 5-6. Expected annual detriments due to the DU exposures shown in Table 5-5. Detriments are considered fatal cancers, nonfatal cancers, and the occurrence of severe heredity effects (ICRP 1990).

\begin{tabular}{|c|c|c|}
\hline Dose to Human Tissue & $\begin{array}{c}\text { Mean } \\
\text { (detriments/yr) }\end{array}$ & $\begin{array}{c}\text { 95\% Confidence Interval } \\
\text { (detriments/yr) }\end{array}$ \\
\hline Kidney & $5.5 \times 10^{-9}$ & $1.4 \times 10^{-9}$ to $1.1 \times 10^{-8}$ \\
Bone & $2.7 \times 10^{-9}$ & $6.5 \times 10^{-10}$ to $5.2 \times 10^{-9}$ \\
GI Tract & $1.9 \times 10^{-10}$ & $4.8 \times 10^{-11}$ to $3.6 \times 10^{-10}$ \\
CEDE & $1.7 \times 10^{-8}$ & $4.3 \times 10^{-9}$ to $3.3 \times 10^{-8}$ \\
\hline
\end{tabular}

Soil concentrations from the environmental monitoring data and our field data were averaged, and the upper bound of the $95 \%$ confidence level, or $23 \mathrm{pCi} / \mathrm{g}$, was used for the simulations. A soil concentration of $35 \mathrm{pCi} / \mathrm{g}$ was used in a second simulation to reflect the concentration specified by the NRC in APG's DU material use license and to reflect the total inventory $(70,000$ to $90,000 \mathrm{~kg})$ of DU dispersed uniformly across the affected area of 820 acres. Both soil concentrations assume uniform distribution of DU in the uppermost $15 \mathrm{~cm}$ of soils. That assumption is demonstrably incorrect as shown in the ERM data and elsewhere (Ebinger et al. 1990). However, modeling a non-uniform source term is problematic, and appropriate codes for predictions based on non-uniform distributions of DU have not been developed. Consequences of using the RESRAD predictions include underestimating doses if soil concentrations are too low and overestimating doses if soil concentrations are too large.

Water concentrations are usually the most sensitive parameters in dose calculations involving aquatic pathways. Therefore, the initial water concentration was important for the RESRAD simulations. Too conservative a value (i.e., too large a concentration) results in unrealistically high doses to animals and 
humans, whereas too low a value results in unrealistically low dose estimates. We used data from ERM sampling and our field data to estimate the initial water concentration of $U$ or DU. RESRAD also allows input of a solubility limit on DU in the soil. The combination of a water concentration based on field data and a DU solubility based on field measurements of dissolved oxygen and Eh (reduction potential) resulted in a realistic initial condition. We set the initial water concentration at the detection limit, or effectively at $0 \mathrm{pCi} / \mathrm{L}$. Through time and in combination with the solubility limit, however, the groundwater and surface water concentrations increased.

Figure 5-1 shows the predicted water concentrations from the present to 100 years in the future for the bounding $\mathrm{DU}$ solubilities in the model of $0.85 \mathrm{pCi} / \mathrm{L}\left(1 \times 10^{-8} \mathrm{~mol} / \mathrm{L}\right.$; Figure $\left.5-1 \mathrm{~A}\right)$ to $850 \mathrm{pCi} / \mathrm{L}(1 \times$ $10^{-5} \mathrm{~mol} / \mathrm{L}$; Figure $5-1 \mathrm{~B}$ ). The bounds of DU solubility in water moving through the soil column were estimated from the geochemical literature supplemented with field data from APG (Tables 3-6 through 38). Langmuir (1978) shows that for Eh and $\mathrm{pH}$ conditions like those measured in APG groundwaters and surface waters, the solubility of DU corrosion products, predominantly schoepite $\left(\mathrm{UO}_{2}(\mathrm{OH})_{2} \bullet \mathrm{H}_{2} \mathrm{O}\right)$, is between $8.5 \times 10^{-3} \mathrm{pCi} / \mathrm{L}$ and $85 \mathrm{pCi} / \mathrm{L}$ (or $10^{-10} \mathrm{~mol} / \mathrm{L}$ and $10^{-6} \mathrm{~mol} / \mathrm{L}$, respectively). The higher solubility limit can be achieved in surface soils at APG especially during dry conditions, whereas the lower solubility limit is more likely in wetter surface soils and in subsurface soils and sediments in the impact area. The solubility range selected for RESRAD simulations, $0.85 \mathrm{pCi} / \mathrm{L}$ to $850 \mathrm{pCi} / \mathrm{L}$, is conservative in that it allows slightly higher concentrations than the limiting thermodynamic conditions actually permit. 

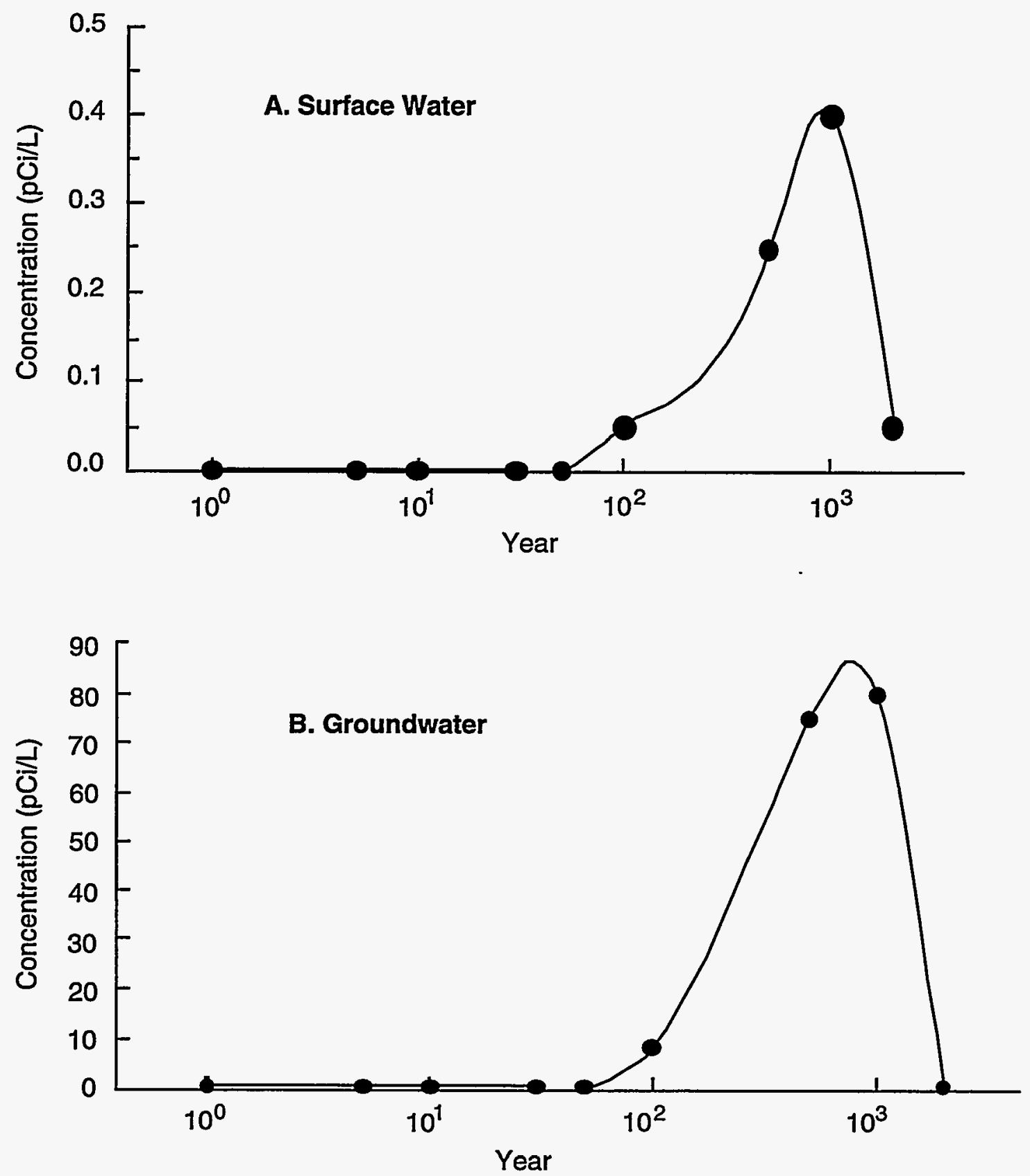

Figure 5-1. Surface water (A) and groundwater (B) concentrations of U at APG predicted with RESRAD. Initial $U$ concentration is $0.85 \mathrm{pCi} / \mathrm{L}$ in $(A)$ and $850 \mathrm{pCi} / \mathrm{L}$ in $(B)$. 
RESRAD models the transfer of radionuclides from soils, fodder, and water to beef and milk from cattle, as well as to plants that provide food for human and animal consumption. We modified the cattle parameters to reflect white-tailed deer by decreasing the amount of fodder and water intake and not allowing milk production for human use. Deer tissue concentrations of $U$ or DU predicted by RESRAD are about $1.2 \times 10^{-3} \mathrm{pCi} / \mathrm{g}$, or about one order of magnitude greater than the concentrations measured in the deer samples (Table 3-14). Given the conservatism in the RESRAD code, this estimate is reasonably close to the measured value for APG deer.

All aquatic pathways were included in the APG simulations, and the amounts of fish and shellfish consumed were the same as those used in the calculations of the doses reported in Tables 5-3 through 5-5. No other modifications of the RESRAD code were made.

Figure 5-2 shows the effect of DU solubility on surface- and groundwater DU concentrations. DU moving through the soil to the groundwater and surface water is retarded for about the first 100 years. After that period, DU is in the water and available to the ecosystem at concentrations as high as about 80 $\mathrm{pCi} / \mathrm{L}$ in the high-solubility simulations. The dose received by a hunter or occasional user of the APG impact area depends on the DU solubility used in the simulation. The simulations at low DU solubility $(0.85 \mathrm{pCi} / \mathrm{L}$ in soil water) are dominated by inhalation of $\mathrm{DU}$-contaminated dust and radiation from the ground for the first 100 years (Figure 5-2), with essentially no contribution to the dose from waterdependent pathways. Doses are higher in the simulations that incorporate higher DU solubility (Figure 53). Doses are similar to those in the the low-solubility simulation during the first 100 years and result from the same two water-independent pathways. After DU enters the water pathway, however, the dose is dominated by ingestion of DU from fish (Figure 5-3). In neither the low-solubility nor the highsolubility simulation is the dose from consumption of deer tissue significant. Doses represented in Figures 5-2 and 5-3 were converted to estimates of risk in terms of detriments, which are shown in Table 5-7. The decrease in the expected detriments with time is the result of DU flushing through the subsurface soil to the water table. Doses and detriments in all simulations increase significantly when drinking water for humans is obtained from either surface- or groundwater or when the land use is changed to resident farming instead of recreational use. The results of resident farming scenarios are not presented in this report, but the magnitude of the doses from resident farming at APG are similar to those from the same scenario in another report (Ebinger and Hansen 1994a).

Soil concentrations of $35 \mathrm{pCi} / \mathrm{g}$ resulted in slightly higher doses than did the lower soil concentration, but the overall trends shown in Figures 5-1 through 5-3 did not change. The maximum dose to humans in the low-solubility simulations was $1.6 \mathrm{mrem} / \mathrm{yr}$ and resulted from ground exposure and inhalation with no contribution from fish and little from deer tissue. The maximum dose to humans in the high-solubility scenario was from consumption of fish and was about $3 \mathrm{mrem} / \mathrm{yr}$. Significant contributions to the dose came from ground exposure and inhalation during the first 100 years, but the dose from fish consumption dominated thereafter. Table 5-7 shows the doses converted to detriments for the $35 \mathrm{pCi} / \mathrm{g}$ soil concentration. 


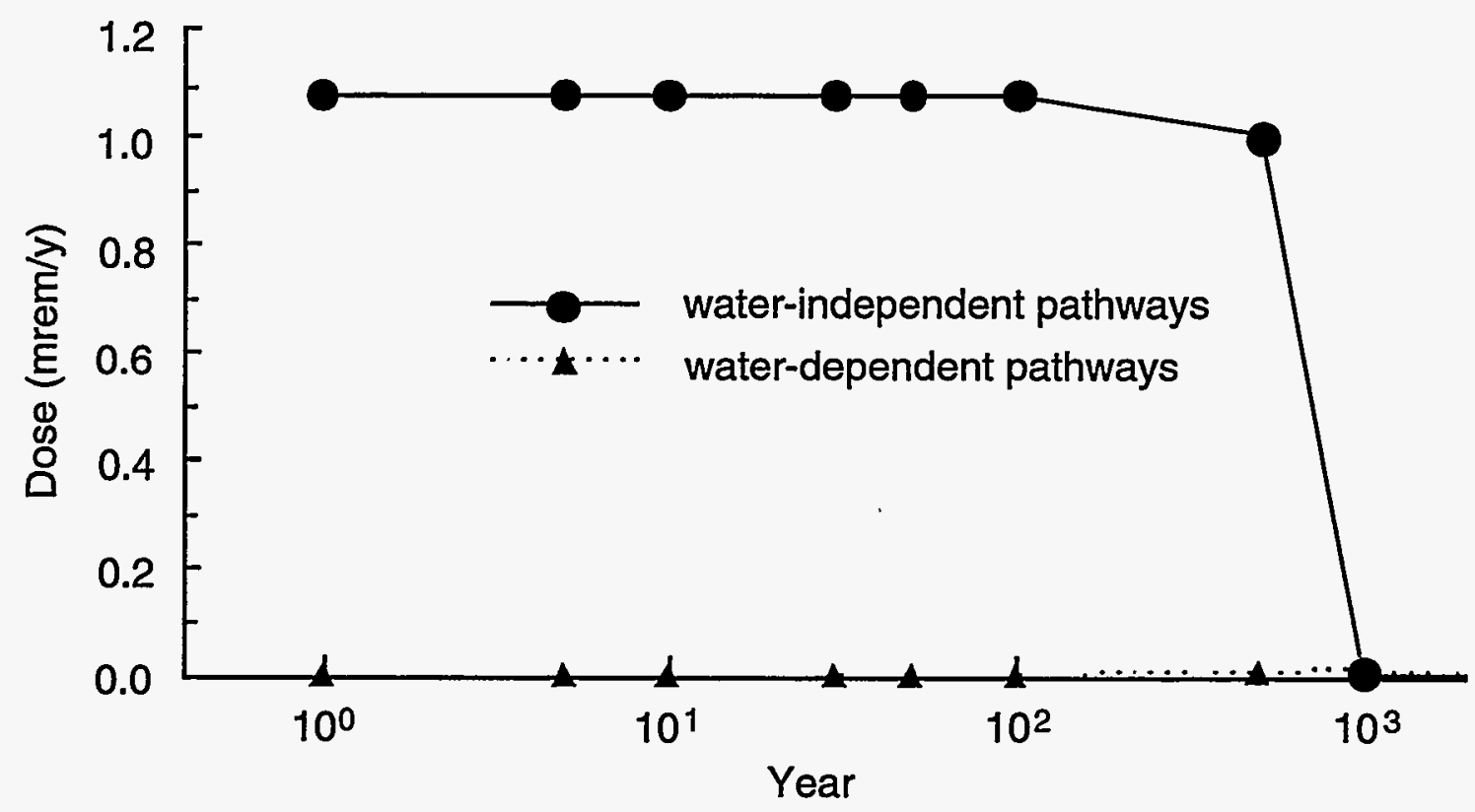

Figure 5-2. Doses from RESRAD with DU solubility of $0.85 \mathrm{pCi} / \mathrm{L}$ (low solubility). Surface water and groundwater are included in the water-dependent pathways line.

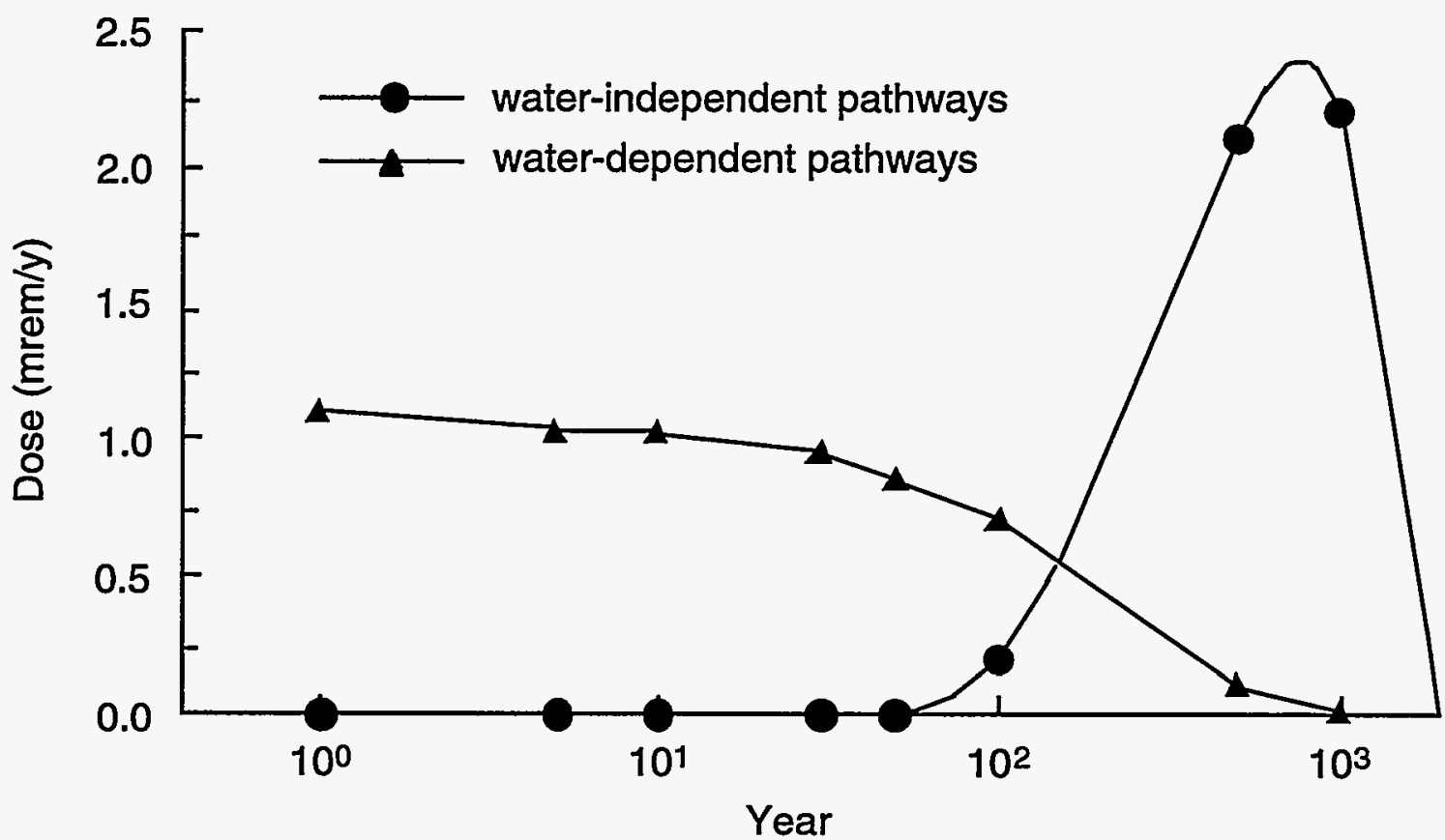

Figure 5-3. Doses from RESRAD with DU solubility of $850 \mathrm{pCi} / \mathrm{L}$ (high solubility). Surface water and groundwater are included in the water-dependent pathways line. 
Table 5-7. Expected detriments from the high-solubility and low-solubility simulations using RESRAD. Values are the number of detriments expected per $10^{6}$ or $10^{5}$ people, as indicated. For example, $7.0 \times 10^{-6}$ indicates there are 7 detriments expected per $10^{6}$ people.

\begin{tabular}{|c|c|c|c|c|}
\hline \multirow{2}{*}{ Year of Simulation } & \multicolumn{2}{|c|}{23 pCi/g Soil Concentration } & \multicolumn{2}{c|}{35 pCi/g Soil Concentration } \\
\cline { 2 - 5 } & $\begin{array}{c}\text { Detriments } \\
\text { (Low Solubility) }\end{array}$ & $\begin{array}{c}\text { Detriments } \\
\text { (High Solubility) }\end{array}$ & $\begin{array}{c}\text { Detriments } \\
\text { (Low Solubility) }\end{array}$ & $\begin{array}{c}\text { Detriments } \\
\text { (High Solubility) }\end{array}$ \\
\hline 0 & $7.0 \times 10^{-6}$ & $7.0 \times 10^{-6}$ & $1.1 \times 10^{-5}$ & $1.1 \times 10^{-5}$ \\
\hline 1 & $7.0 \times 10^{-6}$ & $7.0 \times 10^{-6}$ & $1.1 \times 10^{-5}$ & $1.1 \times 10^{-5}$ \\
\hline 5 & $7.0 \times 10^{-6}$ & $6.8 \times 10^{-6}$ & $1.1 \times 10^{-5}$ & $1.0 \times 10^{-5}$ \\
\hline 10 & $7.0 \times 10^{-6}$ & $6.6 \times 10^{-6}$ & $1.1 \times 10^{-5}$ & $1.0 \times 10^{-5}$ \\
\hline 30 & $7.0 \times 10^{-6}$ & $6.0 \times 10^{-6}$ & $1.1 \times 10^{-5}$ & $9.0 \times 10^{-6}$ \\
\hline 50 & $7.0 \times 10^{-6}$ & $5.4 \times 10^{-6}$ & $1.1 \times 10^{-5}$ & $8.1 \times 10^{-6}$ \\
\hline 100 & $7.0 \times 10^{-6}$ & $5.8 \times 10^{-6}$ & $1.0 \times 10^{-5}$ & $8.8 \times 10^{-6}$ \\
\hline 500 & $7.0 \times 10^{-6}$ & $1.6 \times 10^{-5}$ & $1.0 \times 10^{-8}$ & $2.4 \times 10^{-5}$ \\
\hline 1000 & $9.7 \times 10^{-8}$ & $1.7 \times 10^{-5}$ & $1.0 \times 10^{-5}$ & $2.5 \times 10^{-5}$ \\
\hline 2000 & $9.7 \times 10^{-8}$ & $3.0 \times 10^{-8}$ & $1.0 \times 10^{-8}$ & $5.0 \times 10^{-8}$ \\
\hline
\end{tabular}

\subsubsection{YPG Hunting Scenario}

There is no direct pathway to humans at YPG from hunting as there is at APG, so we developed the following hypothetical scenario as a conservative estimate of human ingestion of DU at YPG: hunters in the area substitute $20 \mathrm{~kg}$ of rabbit for beef each year, and the rabbits use the impact areas extensively for feeding. This scenario is currently not demonstrated at YPG but will serve as an illustration.

Using the results presented in Table 5-2 for consumption of deer tissue, we estimated radiological doses to humans from consumption of rabbit tissue (Table 5-8). Rabbit carcasses were assumed to have a maximum of $1.5 \times 10^{-2} \mathrm{pCi} / \mathrm{g}$ (fresh weight) based on the concentrations measured in rabbit samples collected from YPG. A consumption rate of $20 \mathrm{~kg} / \mathrm{yr}$ and retention rates of $1 \%$ and $5 \%$ of ingested uranium result in the doses shown in Table 5-8. These doses are too low to measure directly, and no adverse human health effects are expected from consumption of rabbit tissue. Likewise, consumption of rabbit tissue results in human kidney burdens of $1.8 \times 10^{-2} \mu \mathrm{g} / \mathrm{g}-\mathrm{kidney}$ if the $20 \mathrm{~kg}$ were consumed uniformly throughout the year. This estimate is well below the most conservative suggested threshold limit for toxicological effects in humans. 
Table 5-8. Doses to humans from consuming rabbit tissue from YPG. Values are within the upper 99\% confidence intervals. Doses were calculated using ingested-DU retention rates of $1 \%$ and $5 \%$.

\begin{tabular}{|c|c|c|}
\hline $\begin{array}{c}\text { Dose to Human } \\
\text { Tissue }\end{array}$ & $\begin{array}{c}\text { Maximum Expected } \\
\text { Dose with 1\% } \\
\text { Retention (mrem/yr) }\end{array}$ & $\begin{array}{c}\text { Maximum Expected } \\
\text { Dose with 5\% } \\
\text { Retention (mrem/yr) }\end{array}$ \\
\hline Kidney & $7.2 \times 10^{-5}$ & $3.6 \times 10^{-4}$ \\
\hline Bone & $1.8 \times 10^{-4}$ & $8.9 \times 10^{-4}$ \\
\hline GI Tract & $2.4 \times 10^{-6}$ & $1.2 \times 10^{-5}$ \\
\hline CEDE & $1.1 \times 10^{-5}$ & $5.3 \times 10^{-5}$ \\
\hline
\end{tabular}

\subsubsection{RESRAD Simulations at YPG}

RESRAD was used to simulate DU transport at YPG in order to project the possible future doses to humans from consumption of rabbit and other game animal tissue. Two soil concentrations were used to bound the simulations, both based on ERM data and field data from YPG. The lower bound was $16 \mathrm{pCi} / \mathrm{g}$ and is the median value of the soil samples collected in the field. The upper bound is $83 \mathrm{pCi} / \mathrm{g}$ and is the mean of the same sample set. Solubility of DU was maintained at $850 \mathrm{pCi} / \mathrm{L}$ in the RESRAD model, which is slightly higher than the upper solubility limit suggested by Langmuir (1978).

The affected area was estimated from the nominal impact area (see Figure 4-1) from field data collected as part of this project, and from separate data reported by Price (1991). We chose as the affected area a rectangle from $4000 \mathrm{~m}$ to $7000 \mathrm{~m}$ downrange from the gun position at GP 20 and $3500 \mathrm{~m}$ to $6500 \mathrm{~m}$ downrange from GP $17 \mathrm{~A}$ and $1000 \mathrm{~m}$ wide on both ranges. The resulting affected area is about $6 \times 10^{6} \mathrm{~m}^{2}$. A depth of $15 \mathrm{~cm}$ was selected for the zone of most of the DU contamination based on previous soil profile information (Ebinger et al. 1990) and field observations made during the current project. We also estimated the average soil concentration by dividing the DU inventory into the volume of soil in the affected area. The resulting soil concentration was $52 \mu \mathrm{g}-\mathrm{U} / \mathrm{g}$ or about $19 \mathrm{pCi} / \mathrm{g}$. Since the estimated soil concentration fell within the bounds calculated from the field samples, no specific RESRAD simulations for the estimated concentration were conducted.

The unsaturated zone between the $15-\mathrm{cm}$ contaminated zone and the water table at about $200 \mathrm{~m}$ was included in the RESRAD simulations. Hydrologic properties for the unsaturated zone were the same as for the contaminated zone. The thickness of the unsaturated zone and rainfall of less than 10 inches per year ensured that DU did not dissolve and migrate to the water table. Instead, the main mechanism of DU transport was erosion from the impact area into adjacent washes.

Depth to groundwater in YPG wells ranges from about 200 to $300 \mathrm{~m}$ from the surface (Entech 1988). No U or DU was detected in samples from wells (Ebinger et al. 1990), and no samples were collected during this project. The depth to the groundwater precludes DU transport by this pathway based on 
current information of groundwater recharge and U migration in arid climates (Scanlon et al. 1990; Phillips et al. 1988). There is no perennial surface water at YPG; thus, the aquatic pathway for RESRAD calculations was not used. Therefore, the only pathways for human exposure were from inhaled dust contaminated with DU, radiation from the ground surface, and consumption of game animals that contained DU from the food chain.

Figure 5-4 shows the total dose from all pathways in the RESRAD simulations at a soil concentration of $16 \mathrm{pCi} / \mathrm{g}$. The dose is dominated by contributions from inhaled dust and direct exposure from the ground surface, and there are contributions of about $1 \%$ from soil ingestion and tissue consumption. The maximum dose received in the RESRAD simulations was about $0.75 \mathrm{mrem} / \mathrm{yr}$. This dose remained constant through the first 100 years of the simulation because it depended only on pathways of physical transport of contaminated soil. Doses decreased to 0 after year 100 because the source area eroded away, thus the DU was dispersed to the washes outside of the impact area. The network of washes extend approximately $15 \mathrm{~km}$ southwest from the affected area and terminate in the confluence with Castle Dome Wash. If the inventory of DU were dispersed evenly along the extent of the washes, the estimated concentration of DU would be far below detection limits using ICP-MS, or much less than $3 \times 10^{-3} \mathrm{pCi} / \mathrm{g}$.

Figure 5-5 shows the RESRAD results for the same simulations but with a soil concentration of 83 $\mathrm{pCi} / \mathrm{g}$. The total dose increased from that in the previous simulation because the soil concentration increased.

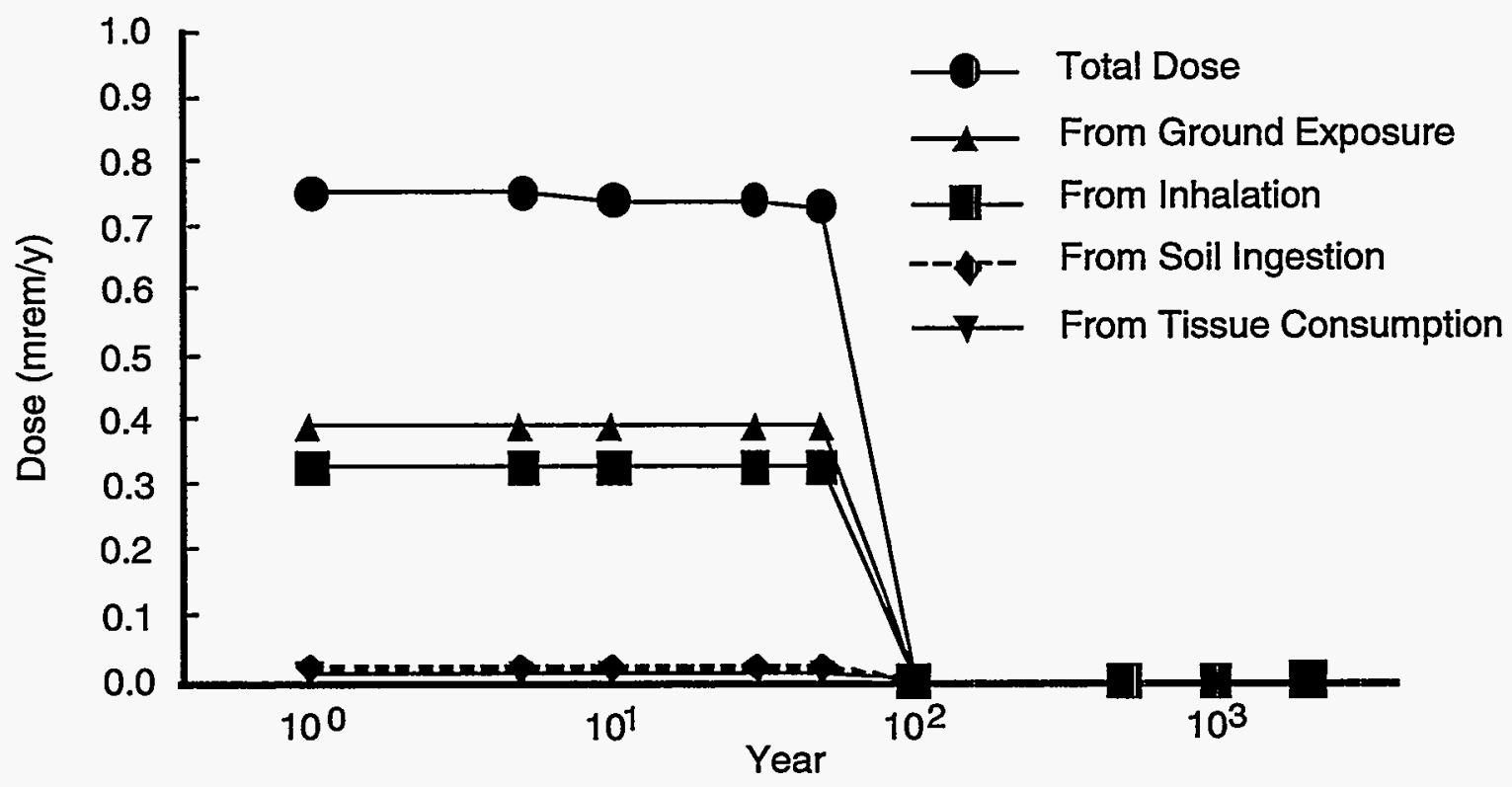

Figure 5-4. Total dose and dose by different pathways from RESRAD simulations with soil $\mathrm{U}$ concentration of $16 \mathrm{pCi} / \mathrm{g}$, YPG. Total dose is the summation of doses from all pathways. 


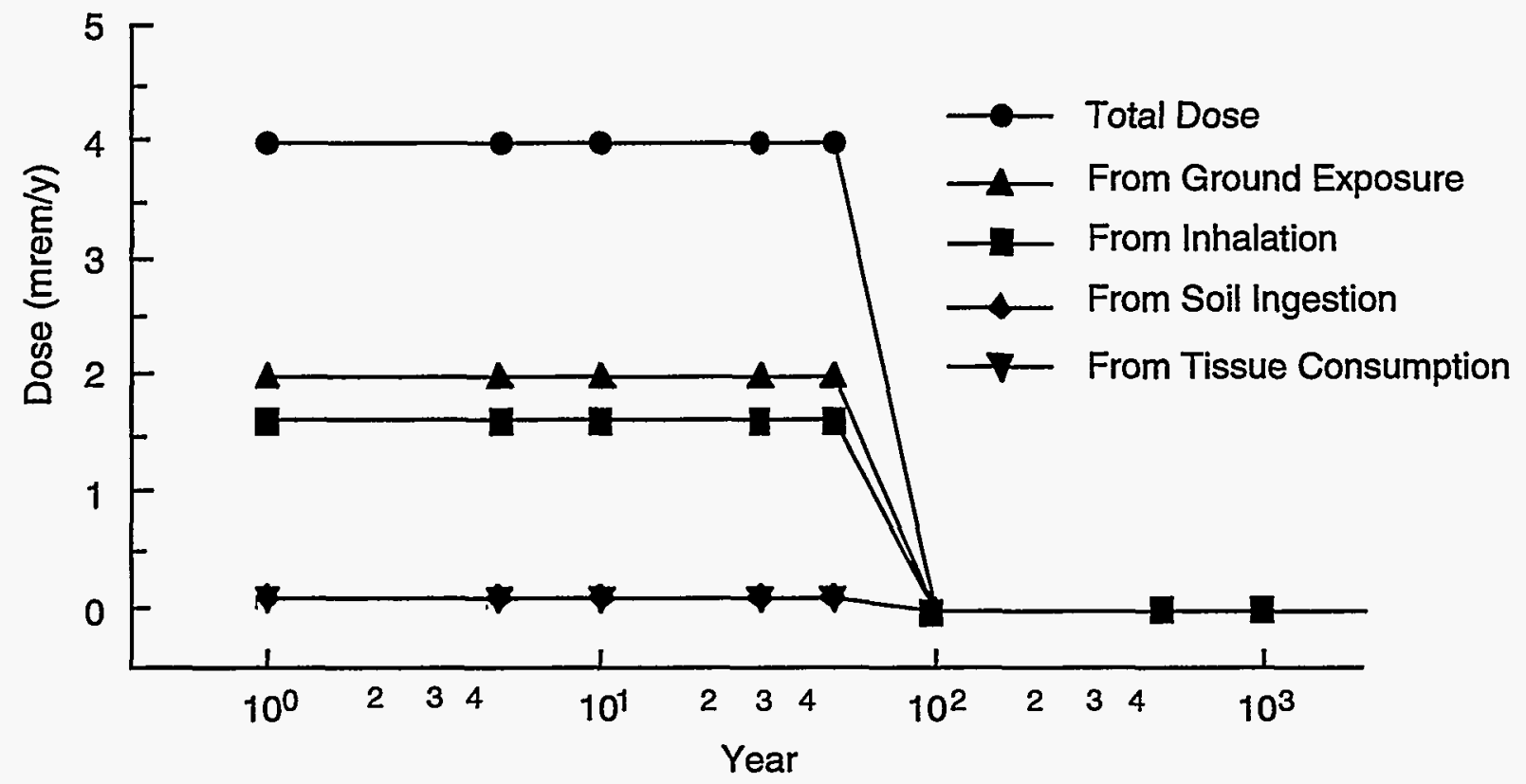

Figure 5-5. Total dose and dose by different pathways from RESRAD simulations of YPG with a soil concentration of $83 \mathrm{pCi} / \mathrm{g}$. Total dose is the summation of doses from all pathways.

The total dose, however, was again largely from ground exposure and inhalation, with very little contribution from tissue consumption. Actual doses are expected to be lower since hunting is not allowed and, therefore, the main exposure pathways are eliminated.

\subsubsection{Toxicological Doses to Humans}

The low specific activity of DU makes it a radiological material of interest, and $U$ andDU are chemical hazards as well. Toxicological effects are not related to the isotopic composition of the U; therefore U and DU are interchangeable in discussions of toxicity. Nephrotoxicity or irreversible damage to the glomerulus occurs at relatively small doses of U or DU (Wrenn et al. 1985; Leggett 1989), and $0.1 \mu \mathrm{g} / \mathrm{g}$ kidney is considered a potential threshold limit for human exposure (Kocher 1989; Zhao and Zhao 1990). We estimated kidney burdens in humans from the expected doses. Table 5-8 shows the expected radiological doses to kidneys. The doses were converted to concentrations and compared with the suggested $0.1-\mu \mathrm{g} / \mathrm{g}$-kidney threshold value. The average kidney burden was $3.4 \times 10^{-2} \mu \mathrm{g}-\mathrm{U} / \mathrm{g}$-kidney (95\% confidence interval of $8.5 \times 10^{-3}$ to $6.6 \times 10^{-2} \mu \mathrm{g}$-U/g-kidney), which is significantly less than the $0.1-\mu \mathrm{g} / \mathrm{g}$-kidney threshold value. Therefore, no adverse chemical toxicity effects are expected.

\subsection{Dose Calculations to Ecosystem Components}

\subsubsection{Methodology}

The International Atomic Energy Agency (IAEA 1992) suggested that radiological dose rates to plants and animals that do not exceed $100 \mathrm{mrad} /$ day ( $1 \mathrm{mGy} /$ day) should not cause long-term adverse effects for animal and plant reproductive capabilities. We adopted this suggested dose rate limit for 
evaluating the doses to plants and animals from exposure to DU. The deer model was adapted from a model of radionuclide uptake by beef and dairy cattle (NCRP 1984a), and was used to calculate the radiological doses to deer from consuming water, soil, and vegetation in the DU impact area and the radiological dose to humans from consumption of deer tissue. DU ingestion by deer occurs from vegetation that contains DU from root uptake as well as DU deposited via resuspension, DUcontaminated drinking water, direct ingestion of contaminated soil, and inhalation of DU-containing dust. Inhalation of dust was omitted from consideration in the dose assessment because soil ingestion and ingestion of DU from plants were one to two orders of magnitude greater than the doses to deer from inhalation. The DU concentration in deer tissue is estimated by Equations 3-16 through 3-23.

We also collected 30 deer for analysis of liver, kidney, muscle, and bone tissues for U or DU. Twenty-five deer were harvested from the impact area and five were harvested from the Eastern Shore of Maryland at sites separated from APG by Chesapeake Bay. The data from deer samples allowed partial validation of the steady-state model as well as a direct method for calculating radiological doses to the tissues of interest and the kidney burden of $U$ or DU.

Estimates of parameters for input to the steady-state model were obtained from field data and the literature, as discussed in Chapter 3. Predictions were made in the same manner as the human health predictions. That is, the uncertainty of the parameter estimates was included in the predictions of concentrations and doses in the animals of interest. The resulting predictions were probability distributions instead of point estimates.

The steady-state model was also used to estimate the dose to deer due to ingestion of DU from vegetation, soil, and water. Dose rates were calculated from the U or DU concentration in the three pathways that make up the deers' food sources. Equation 3-16 was used to determine the DU concentration ingested by deer, and then the concentrations were converted to dose rates $(D)$ using

$$
D=C_{i a} E f C F \quad,
$$

where $C_{i \mathrm{a}}$ is from Equation 3-16, $E$ is the energy of $\alpha$-disintegration for ${ }^{238} \mathrm{U}$ (4.2 MeV and 4.15 MeV), $f$ is the fraction of the corresponding energy ( 0.75 for $4.2 \mathrm{MeV}, 0.25$ for $4.15 \mathrm{MeV}$ ), and $C F$ is $5.12 \times 10^{-5}$ and converts $D$ to $\mathrm{mrad} /$ day. The median dose rate to deer due to DU ingestion was $1.7 \times 10^{-1} \mathrm{mrad} / \mathrm{day}$ with a $95 \%$ confidence interval of $2.8 \times 10^{-3}$ to $17.3 \mathrm{mrad} / \mathrm{day}$. The estimated dose rates are well below the $100 \mathrm{mrad} /$ day suggested by the IAEA (1992) to limit adverse effects in animal and plant populations.

Field samples of sunfish (Centrarchidae) and perch (Perca flaveocene and Morone americanus) were collected in sufficient quantity to estimate distribution of the $U$ and DU concentrations in tissue (Figures 3-8 and 3-9). These distributions were used to estimate the radiological dose to humans from consumption of about $7 \mathrm{~kg}$ of fish per year (NCRP 1984a), as shown in Table 5-3. The same distributions were used to estimate the dose rates to perch and sunfish using Equation 5-1. 


\subsubsection{Assumptions}

The steady-state model requires several assumptions before it can be used for predicting radiological dose rates or the toxicological effects of DU exposure. The values of the parameters are listed in Appendix B and were discussed in Chapter 3.

Concentration data for the compartments and species were taken from the field samples. Body masses were also required for the risk estimates for the ecosystem compartments, and additional field data on carcass weights, body masses, and organ masses were used (Arthur and Gates 1988; Lautier et al. 1988; Arthur and Alldredge 1979; Alldredge et al. 1974). Concentration data and body mass data were used as distributions so that uncertainty in the estimates could be calculated. The uncertainty in the predicted doses and subsequent risk of adverse effects also include the uncertainty inherent in the measurements or assumptions used in previous calculations.

\subsubsection{Results: Ecosystem Risk Assessment}

Overall, radiological doses and toxicological effects were of minimal consequence, as indicated by the analyses of the field data described in Chapters 3 and 4. Data from the field sampling campaign were used to estimate the dose rates to ecosystem compartments. Table 5-9 shows the predicted dose rates to specific compartments and/or species and the $95 \%$ confidence intervals of the estimates.

\subsubsection{Deer}

The mean dose rate to deer calculated from the field data was about $1 \times 10^{-6} \mathrm{mrad} /$ day with a $95 \%$ confidence interval from $9.4 \times 10^{-7} \mathrm{mrad} /$ day to $1.3 \times 10^{-6} \mathrm{mrad} /$ day (Table 5-9). The $95 \%$ confidence interval of predicted deer tissue concentration was $1.0 \times 10^{-4} \mathrm{mrad} /$ day to $3.3 \times 10^{-4} \mathrm{mrad} /$ day, reflecting the added uncertainty in the predicted value. The distribution of the predictions are highly skewed toward concentrations lower than the mean. Dose rates calculated for deer using either field data or predicted values are well below the $100 \mathrm{mrad} /$ day suggested by the IAEA (1992). Deer kidney data (Table 3-14) also were used to estimate the kidney burden of DU. Kidney burdens calculated from field data range from $7 \times 10^{-4}$ to $1.6 \times 10^{-3} \mu \mathrm{g} / \mathrm{g}$-kidney and average about $1.2 \times 10^{-3} \mu \mathrm{g} / \mathrm{g}$-kidney. This kidney burden does not appear to adversely affect the function of deer kidneys based on the suggested toxicity of $1 \mu \mathrm{g} / \mathrm{g}$ kidney (fresh weight).

\subsubsection{Sunfish and Perch}

The average predicted dose to sunfish at APG was $1.3 \times 10^{-4} \mathrm{mrad} /$ day and the $95 \%$ confidence interval was $1.2 \times 10^{-4} \mathrm{mrad} /$ day to $1.4 \times 10^{-4} \mathrm{mrad} /$ day (Table 5-9). The average predicted dose to perch at APG was $1.6 \times 10^{-5} \mathrm{mrad} /$ day and the $95 \%$ confidence interval was from the detection limit to $4.8 \times 10^{-5} \mathrm{mrad} / \mathrm{day}$ (Table 5-9). The small confidence intervals can be accounted for by the distribution of body masses and concentrations used to calculate the dose estimates. Dose rates to blue crabs were also low, with the $95 \%$ confidence interval from $2.6 \times 10^{-3}$ to $5 \times 10^{-3} \mathrm{mrad} /$ day (Table 5-9). The data and the calculated dose rates show that there is little support for hypotheses proposing that exposure to $U$ and/or DU at APG results in significant adverse effects on these aquatic ecosystem components. 


\subsubsection{Kangaroo Rats and Pocket Mice}

Dose rate calculations using YPG data are similar to results from APG. Dose rates to kangaroo rats were low, with the $95 \%$ confidence interval from $4.9 \times 10^{-4}$ to $5.2 \times 10^{4} \mathrm{mrad} /$ day. Dose rates to pocket mice were also low, with the $95 \%$ confidence interval from $6.9 \times 10^{-3}$ to $7.5 \times 10^{-3} \mathrm{mrad} /$ day. The upper bound of the $95 \%$ confidence interval on kidney burdens in kangaroo rats, however, suggests the potential for adverse nephrotoxic effects.

The histopathology investigation suggested that renal function in kangaroo rats and possibly pocket mice and other members of the small herbivore compartment for the YPG model could be at risk. Renal function of terrestrial biota at APG could be similarly affected. However, our sampling scheme concentrated on the aquatic ecosystem and placed no emphasis on the histopathology of terrestrial mammals. Continued monitoring of the small herbivore compartment for DU at both APG and YPG is recommended and could be incorporated into the respective environmental monitoring plans.

Table 5-9. Dose rates calculated from concentrations of $U$ and DU in various compartments of the APG and YPG ecosystems.

\begin{tabular}{|c|c|c|c|c|c|}
\hline & & & & \multicolumn{2}{|c|}{ 95\% Confidence Interval } \\
\hline $\begin{array}{c}\text { Compartment } \\
\text { or Species }\end{array}$ & $\begin{array}{c}\text { Average } \\
\text { Concentration } \\
\text { (pCi/g-fresh } \\
\text { weight) }\end{array}$ & $\begin{array}{c}\text { Average Body } \\
\text { Mass (g-fresh } \\
\text { weight) }\end{array}$ & $\begin{array}{c}\text { Average Dose } \\
\text { Rate } \\
\text { (mrad/day) }\end{array}$ & Lower Bound & Upper Bound \\
\hline \multicolumn{6}{|c|}{ APG Compartments } \\
\hline Sunfish & $3.1 \times 10^{-3}$ & $3.1 \times 10^{1}$ & $1.3 \times 10^{-4}$ & $1.2 \times 10^{-4}$ & $1.4 \times 10^{-4}$ \\
\hline Perch & $2.3 \times 10^{-3}$ & $1.2 \times 10^{1}$ & $1.6 \times 10^{-5}$ & DL & $4.8 \times 10^{-5}$ \\
\hline Deer & $3.6 \times 10^{-4}$ & $3.4 \times 10^{4}$ & $1.0 \times 10^{-6}$ & $9.4 \times 10^{-7}$ & $1.3 \times 10^{-6}$ \\
\hline Blue Crab & 3.6 & $3.0 \times 10^{2}$ & $3.8 \times 10^{-3}$ & $2.7 \times 10^{-3}$ & $5.0 \times 10^{-3}$ \\
\hline \multicolumn{6}{|c|}{ YPG Compartments } \\
\hline Kangaroo Rat & $4.1 \times 10^{-2}$ & $3.3 \times 10^{1}$ & $5.1 \times 10^{-4}$ & $4.9 \times 10^{-4}$ & $5.2 \times 10^{-4}$ \\
\hline Pocket Mice & 4.8 & $2.6 \times 10^{1}$ & $7.2 \times 10^{-3}$ & $6.9 \times 10^{-3}$ & $7.5 \times 10^{-3}$ \\
\hline Lizards & 3.3 & $6.1 \times 10^{1}$ & $3.1 \times 10^{-3}$ & $\mathrm{DL}$ & $4.8 \times 10^{-3}$ \\
\hline Rabbit & $3.6 \times 10^{-3}$ & $1.5 \times 10^{3}$ & $1.6 \times 10^{-6}$ & $1.5 \times 10^{-6}$ & $1.6 \times 10^{-6}$ \\
\hline Insects & 3.4 & 2.0 & $9.1 \times 10^{-2}$ & DL & $2.3 \times 10^{-1}$ \\
\hline
\end{tabular}




\subsubsection{Invertebrates}

Insects show radiological dose rates with $95 \%$ confidence intervals from the detection limit to $2.3 \times$ $10^{-1} \mathrm{mrad} /$ day. This was the highest dose rate predicted from the field data and still well below the suggested guideline (IAEA 1992). Concentrations of DU were consistently measured in the field samples and this compartment is important in the APG and YPG food chains, so sampling the invertebrate compartment is recommended for inclusion in the environmental sampling programs.

\subsubsection{Insectivores (Lizards)}

There was no predicted adverse effects on the lizards (insectivores compartment) that would suggest the insects are propagating the DU through that part of the food web. The dose rate for lizards had a $95 \%$ confidence interval from the detection limit to $4.8 \times 10^{-3} \mathrm{mrad} /$ day. The upper bound of the interval represents a factor-of-100 reduction in the highest concentration found in the insects. Surface coatings of DU-containing dust were a probable source of DU to consumers of lizards in the food chain. The dose rates to different consumers of lizards were not greater than about $1 \mathrm{mrad} /$ day.

\subsubsection{Large Herbivores}

Large herbivores (rabbits) showed low dose rates with a $95 \%$ confidence interval of $1.5 \times 10^{-6}$ to 1.6 $\times 10^{-3} \mathrm{mrad} /$ day. Dose rates are low partly because of the larger body mass of the rabbits and the low exposure from ingestion and soil incorporated in pelts. The sample size is relatively small (10 individuals) and would benefit from additional data collected as part of continued environmental monitoring. Body burdens of only two predators were analyzed, and the highest concentration (2.9 $\mathrm{pCi} / \mathrm{g}$ ) gives a dose rate of about $4 \times 10^{-3} \mathrm{mrad} /$ day. The sample size was too small to support conclusions concerning the dose rate to a larger population of predators. Also, sampling of additional species of predators should be conducted to better estimate exposure to DU and to evaluate kidney burdens in this compartment. The results of analyses of samples from the other compartments of the YPG model suggest that the predators are not at risk to adverse effects from DU ingestion.

\subsection{Conclusions}

The goal of the work reported in this document was to provide information from APG and YPG to address the following questions:

- Is DU migrating through soils of the impact areas at APG and YPG?

- Is DU migrating into Chesapeake Bay at APG or off-site at YPG?

- Does DU adversely affect ecosystems at APG or YPG?

- Does residual DU at APG or YPG adversely affect human health?

The data presented in Chapters 3 and 4 and the dose estimates and risk evaluations in this chapter support the following conclusions. 


\subsection{1. $U$ and DU Transport}

Previous studies at APG and YPG showed that DU moves through soil profiles after penetrator fragments are deposited in the environment (Ebinger et al. 1990; Ward and Stevens 1994). Data from soils at APG and YPG collected during this project and during environmental monitoring confirms the non-uniform distribution of DU in soils. Deposited DU fragments are potentially mobile in the soils. At APG rainfall provides sufficient moisture to transport corrosion products from fragments through soil profiles to depths of at least $20 \mathrm{~cm}$ and probably deeper. Data from groundwater wells in the impact area, however, show no effects of DU transport. This trend could change with time because DU transport is slow and may require many years to reach the sampling volume of the monitoring wells; no DU has been detected, however, in nearly 25 years of DU testing at APG.

Subsurface transport of DU at APG is hindered for several reasons. First, the groundwater flow velocity is low due to the low gradient in the aquifer. Second, high water tables at APG are common, as seen in the mottled gray soil profiles throughout the impact area and the frequently observed ponds. High water tables support reducing conditions in these soils. Measurements of dissolved oxygen and oxidation-reduction potential confirm that reducing conditions in wet soils are prevalent, and there is additional visual evidence from soil mottling that shows microbial activity in the soil is sufficient to reduce iron from $\mathrm{Fe}^{3+}$ to $\mathrm{Fe}^{2+}$ and $\mathrm{U}^{6+}$ to $\mathrm{U}^{4+}$ (Lovley et al. 1991). Uranium in the 4+ state is about three orders of magnitude less soluble than $\mathrm{UO}_{2}{ }^{2+}$, the common form of soluble $\mathrm{U}$ in the $6+$ state (Langmuir 1978). Thus, there is a high probability that DU mobility is limited by the precipitation of $U^{4+}$ solids within the wet, anaerobic soils and in the wetlands surrounding APG. There is also high probability that precipitation of $U^{4+}$ is moderated by microbial activity, as indicated in other studies (Lovley et al. 1991). Third, most of the DU is U metal in the penetrator fragments, which is slowly corroding and being released to the soil-water system (Erikson et al. 1993). The amount of DU entering the soil system depends on the corrosion rate of the fragments, and field data suggest that DU corrodes slowly (Erikson et al. 1993, 1990a, 1990b; Grandstaff 1976). The inventory of DU in the soils is, therefore, released and available to biota over a long time period and at low concentrations and is not available to plants and animals all at once.

Erosion at YPG is the primary mechanism of DU transport. DU in washes adjacent to impact areas has been observed (Ebinger et al. 1990), but attempts to systematically sample DU in washes have met with little success. Ward and Stevens (1994) showed that desert pavements provide necessary conditions for collecting the limited rainfall at YPG and channeling it to cause erosion. However, DU movement by way of erosion is limited because most desert pavements have insufficient gradients to sustain runoff except that from the most intense storms. While some of the inventory will erode into the washes, the majority of the material will be retained on desert pavements. Reducing conditions in the soils are rare at YPG, and DU transport is not limited by precipitation of $\mathrm{U}^{4+}$ solids as it is at APG. However, the low annual rainfall limits dissolution of DU and thereby limits chemical transport of DU into the soil profiles. While the corrosion rates of DU fragments seem to be greater at YPG, the potential to transport the corrosion products depends on the amount and timing of rain. High-intensity storms are required to 
create surface flow conditions sufficient to move DU fragments on desert pavement. Appropriate rainfall events are infrequent in the summer and occur rarely at other times during the year.

Wind deposition of DU is a secondary mode of transport for DU at YPG. Wind dispersal of dust that contains DU was observed in the field during penetrator testing, and wind dispersal is most prevalent during testing. Wind across the impact areas when no testing occurs does not disturb DU-containing dust as frequently as firing does. Thus, wind dispersal without simultaneous munitions testing is a minor DUtransport mechanism. DU-containing dust was observed as coatings on vegetation surfaces during field surveys and on laboratory samples.

DU corrosion products are transported through the soils in the impact areas at APG and YPG, and the affected areas are the soil volumes within which the penetrator fragments are deposited. Transport through the soils to groundwater and surface water is theoretically possible at APG, but reducing conditions in the soils and groundwater and slow groundwater flow rates mean that most of the inventory is retained in the soils. Transport through the 200- to300-m unsaturated zone at YPG may occur, but transport by erosion is considerably more rapid. Transport of DU off-site to Chesapeake Bay at APG or to the Gila and Colorado Rivers at YPG is unlikely because of the long time required for transport from the source region into drainage networks that eventually empty into the Bay or rivers.

Data in the previous sections suggest that ecosystem compartments are exposed to DU from penetrators, but few are adversely affected by the DU. The only data supporting possible adverse effects were collected from kangaroo rat histopathological investigations after controlled-dose experiments, and the only predicted doses that suggest adverse effects due to U or DU ingestion were from kangaroo rat and pocket mouse kidneys.

The main pathways by which DU may be transported from the impact areas are through the detritus and water pathways at APG and erosion at YPG. Detritus may have DU particles adsorbed onto it or complexed with it, or it may act as a support for suspended DU. Filtration of large detritus/DU complexes by soils in the wetlands will limit the amount of detritus that is transported from the impact areas. Continued monitoring of water and aquatic plants and animals will track over time the transport of DU in the detritus.

Erosion of soils at YPG deposits DU and sediments from runoff into washes that dissect the impact areas, and the washes are potential conduits for moving DU off-site. Samples of sediments from washes included several with $U$ concentrations below detection limits, and DU was detected in wash sediments only where it was observed visually, that is, within about $50 \mathrm{~m}$ of impact areas. The volume of "clean" sediment carried by washes masks DU or dilutes it to below detection limits, effectively decreasing the concentration of DU transported off-site. The tortuosity of the washes provides numerous depositional locations within the YPG reservation for collection of DU eroding from impact areas. The availability of depositional areas within washes that act as DU sinks supports the low probability of high concentrations of DU moving off-site. Several small washes drain the impact area, converge with larger washes, and ultimately converge with Castle Dome Wash. Castle Dome Wash runs from north to south across the YPG reservation and drains into the Gila River. DU eroded into the small washes must be transported 
through a series of smaller washes before reaching Castle Dome Wash, and sufficient flow in the washes must exist before transport occurs. Limited rainfall of sufficient intensity to cause channel flow limits the distance of DU transport. Thus, the probability of DU moving off YPG boundaries is low and depends on how often all of the washes flow.

\subsubsection{Ecosystem Effects from Exposure to DU}

We show data that support little if any adverse effect on the ecosystems at APG and YPG. At YPG the small herbivore compartment (pocket mice and kangaroo rats, in particular) shows possible effects due to the chemical toxicity of $U$ on the kidney. Histopathology of kangaroo rat kidneys suggests some deterioration of the kidneys, and this damage could correlate to stress on kangaroo rat populations at YPG. The histopathology results should be confirmed by additional controlled laboratory studies to ensure that the effects are specifically from DU and to confirm the concentrations at which the effects occur. Adverse effects due to radiation from DU does not appear likely according to our data and calculations of doses to animals and plants.

No adverse radiation or toxicological effects were suggested in the data collected at APG even though we found $U$ in deer kidneys. Radiation doses and toxicological exposures were estimated, but conservative estimates indicate that the ecosystem components we tested are at no elevated risk of damage due to DU. It should be noted that attributing the source of $U$ to DU is not supported or refuted by many data presented in this report, though isotopic ratios of some samples show clearly that elevated $\mathrm{U}$ is from DU deposited during munitions testing.

\subsubsection{Human Health Effects}

As with ecosystem compartments, our data suggest that minimal adverse human health effects from DU are expected. There is at least one pathway to humans at APG from the deer hunting program, but predictions including this pathway indicate that radiological and toxicological doses are well below threshold limits. In our simulations, combined fishing, hunting, and consumption of contaminated water do not contribute enough DU to cause adverse human health effects when the predictions are made with data collected in the field and data from environmental monitoring reports.

There is no direct pathway to humans at YPG, but a possible scenario involving rabbit consumption by hunters was used to evaluate potential human health risk. As with the APG deer hunting, predictions that include the consumption of rabbits from YPG did not adversely affect human health from a radiological or toxicological standpoint.

This report shows that $U$ and DU can be measured in different ecosystem compartments at APG and YPG. The data and predictions of health effects based on the data, however, show that most compartments sampled are not adversely affected by natural $U$ or residual DU in the impact areas. Potential kidney damage in kangaroo rats at YPG may indicate significant ecological impacts from DU munitions testing. Laboratory testing of DU uptake in kangaroo rats is recommended along with ongoing environmental monitoring. No sampling except for deer was conducted to evaluate similar effects in 
terrestrial animals at APG, and subtle adverse effects may have been overlooked. The data in this report suggest that adverse effects from radiation or toxicity of DU were not common or were not observed in most compartments. The lack of data on DU concentrations and renal function of terrestrial animals other than deer at APG suggests that additional sampling should continue as part of the environmental radiation monitoring program. Continued analysis of environmental monitoring data will provide longer-term information that will demonstrate the presence or absence of significant ecological impact due to DU testing. This report suggests that the residual DU in the environment at present does not result in adverse effects to the ecosystem compartments sampled and that future adverse effects should be small.

\section{Acknowledgments}

This project was completed with funding from the U.S. Army Test and Evaluation Command. We thank Tanya Oxenberg, Larry Davis, and John Beckman of APG and Frank Dunfrund, Lance Vander Zyl, Valorie Morrill, and John McCabe of YPG for their assistance in field investigations and during the course of this work. We also thank Edward H. Essington (EES-15) for his thoughtful and thorough review of the manuscript of this report. 



\section{REFERENCES}

Ahsanullah, M., and A.R. Williams. Effect of uranium on growth and reproduction of the marine amphipod Allorchestes compressa. Marine Biology 93:459-464 (1986).

Ahsanullah, M., and A.R. Williams. Kinetics of uranium uptake by the crab Pachygrapsus laevimanus and the zebra winkle Austracochlea constricta. Marine Biology 101:323-327 (1989).

Alldredge, A.W., J.F. Lipscomb, and F.W. Whicker. Forage intake rates of mule deer.estimated with fallout cesium-137. J.Wildl. Manage. 38:508-516 (1974).

Amiel, S. Analytical applications of delayed neutron emission in fissionable elements. Analytical Chemistry 34:1683-1692 (1962).

Anderson, R.F., M.Q. Fleisher, and A.P. LeHuray. Concentration, oxidation state, and particulate flux of uranium in the Black Sea. Geochimica et Cosmochimica Acta 53:2215-2224 (1989a).

Anderson, R.F., A.P. LeHuray, M.Q. Fleisher, and J.W. Murray. Uranium deposition in Saanich Inlet sediments, Vancouver Island. Geochimica et Cosmochimica Acta 53:2205-2213 (1989b).

Anspaugh L.R., J.H. Shinn, P.L. Phelps, and N.C. Kennedy. Resuspension and redistribution of plutonium in soils. Health Physics 29:571-582 (1975).

Arditi, R., and H. Saiiah. Empirical evidence of the role of heterogeneity in ratio-dependent consumption. Ecology 73:1544-1551 (1992).

Arthur, W.J.,III, and A.W. Alldredge. Soil ingestion by mule deer in northcentral Colorado. J. Range Manage. 32:67-70 (1979).

Arthur, W.J.,III, and R.J. Gates. Trace element intake via soil ingestion in pronghorns and in black-tailed jackrabbits. J. Wildl. Manage. 32:67-70 (1988).

Baird, D., and R.E. Ulanowicz. The seasonal dynamics of the Chesapeake Bay ecosystem. Ecological Monographs 59(4):329-364 (1989).

Barnthouse, L.W. The role of models in ecological risk assessment: a 1990's perspective. Environmental Toxicology and Chemistry 11:1751-1760 (1992).

Barnthouse, L.W., J. Boreman, S.W. Christensen, C.P. Goodyear, W. Van Winkle, and D.S. Vaughan. Population biology in the courtroom: the Hudson River controversy. BioScience 34:14-19 (1984).

Bartell, S.M., R.H. Gardner, and R.V. O'Neill. Ecological risk estimation. Lewis Publishers, Chelsea, MI (1992).

Begon, M., J.L. Harper, and C.R. Townsend. Ecology: individuals, populations and communities. Blackwell Scientific Publications, Boston, MA (1990).

Benninger, L.K., R.C. Aller, J.K. Cochran, and K.K. Turekian. Effects of biological sediment mixing on the ${ }^{210} \mathrm{~Pb}$ chronology and trace metal distribution in a Long Island Sound sediment core. Earth Planet. Sci. Let. 43:241-259 (1979).

Berryman, A.A. The origins and evolution of predatory-prey theory. Ecology 73:1530-11535 (1992).

Blaylock, B.G., and J.P. Witherspoon. Evaluation of radionuclides released from the light water reactor nuclear fuel cycle to the aquatic environment. Environmental Chemistry and the Cycling Process. Symposium in Augusta, Georgia. CONF-760429 NTIS (1976).

Bowie, G.L., W.B. Mills, D.B. Porcella, C.L. Campbell, J.R. Pagenkopf, G.L. Rupp, K.M. Johnson, P.W.H. Chan, S.A. Gherini, and C.E. Chamberlin. Rates, constants, and kinetics formulations in surface water quality modeling. Environmental Protection Agency Report EPA/600/3-85/040 (1985).

Brenchley, D.L., J.K. Soldat, J.A. McNeese, and E.C. Watson. Environmental assessment methodology of the nuclear fuel cycle. Battelle Pacific Northwest Laboratories Report BNWL-2219 (1977). 
Breshears, D.D., T.B. Kirchner, and F.W. Whicker. Contaminant transport through agroecosystems: assessing relative importance of environmental, physiological, and management factors. Ecological Applications 2:285-297 (1992).

Brown, D.E., Editor. Biotic communities of the American Southwest-United States and Mexico. Desert Plants 4:1-342 (1982).

Brown, J.H. and E.J. Heske. Control of a desert-grassland transition by a keystone rodent guild. Science 250:1705-1707 (1990).

Calder, W.A. Size, function, and life history. Harvard University Press, Cambridge, MA (1984).

Chew, R.M., and A.W. Chew. Energy relationships of the mammals of a desert shrub Larrea tridentata community. Ecological Monographs 40:1-21 (1970).

Coleman, R.F., and T.B. Pierce. Activation analysis: A review. Analyst 92(1090):1-19 (1967).

Cothern, C.R., W.L. Lappenbusch, and J.A. Cotruvo. Health effects guidance for uranium in drinking water. Health Physics 44 (Supplement 1):377-384 (1983).

Cothern, C.R., and W.L. Lappenbusch. Occurrence of Uranium in Drinking Water in the U. S. Health Physics. 45:89-99 (1983).

Crosswhite, F.S., and C.D. Crosswhite. The Sonoran Desert. In G.L. Bender, Editor, Reference handbook on the deserts of North America. Greenwood Press, Westport, CT, pp. 163-319 (1982).

DOE (Department of Energy). Internal Dose Conversion Factors for Calculation of Dose to the Public. U.S. DOE Report DE88-014297 (1988).

Dreicer, M., T.E. Hakonson, G.C. White, and F.W. Whicker. Rainsplash as a mechanism for soil contamination of plant surfaces. Health Physics 46:177-187 (1984).

Ebinger, M.H., E.H. Essington, E.S. Gladney, B.D. Newman, and C.L. Reynolds. Long-term fate of depleted uranium at Aberdeen and Yuma Proving Grounds Final Report, Phase I: Geochemical transport and modeling. Los Alamos National Laboratory Report LA-11790-MS (1990).

Ebinger, M.H., and W.R. Hansen. Depleted Uranium Human Health Risk Assessment, Jefferson Proving Ground, Indiana. Los Alamos National Laboratory Report LA-UR-94-1809 (1994a).

Ebinger, M.H., and W.R. Hansen. Environmental Radiation Monitoring Plan for Depleted Uranium and Beryllium Areas, Yuma Proving Ground. Los Alamos National Laboratory Report LA-UR-941838 (1994b).

Ebinger, M.H., and W.R. Hansen. A consolidated Environmental Monitoring Plan for Aberdeen Proving Ground, Maryland. Draft Los Alamos National Laboratory Report (1995).

Entech. Geohydrologic Study of the Yuma Proving Ground with Particular Reference to the Open Burning/Open Detonation Facility. Entech Engineers, Santa Ana, CA (1988).

EPA (Environmental Protection Agency). "Method 200.2: Sample Preparation Procedure for Spectrochemical Determination of Total Recoverable Elements," in Methods for the Determination of Metals in Environmental Samples. EPA Publication EPA/600/4-91/010 (1991).

EPA. "Method 3050 Acid Digestion of Soils, Sediments, and Sludges," in Test Methods for Evaluating Solid Waste: Physical/Chemical Methods. EPA Publication SW846 (1987).

Erikson, R.J., C.J. Hostetler, R.J. Serne, J.R. Divine, and M.A. Parkhurst. Geochemical Factors Affecting Degradation and Environmental Fate of Depleted Uranium Penetrators in Soil and Water. Pacific Northwest Laboratory Report PNL-8527 (1993).

Erikson, R.L., C.J. Hostetler, J.R. Divine, and K.R. Price. A review of the environmental behavior of uranium derived from depleted uranium alloy penetrators. Pacific Northwest Laboratory Report PNL-7213 (1990a). 
Erikson, R.L., R.J. Serne, J.R. Divine, and K.R. Price. Measured and theoretical behavior of depleted uranium corrosion products in soil systems. Prepared by the Pacific Northwest Laboratory for the U.S. Army Combat Systems Test Activity, Aberdeen Proving Ground, MD (1990b).

Ginzburg, L.R., and H.R. Akçakaya. Consequences of ratio-dependent predation for steady-state properties of ecosystems. Ecology 73:1536-1543 (1992).

Gladney, E.S., W.D. Moss, M.A. Gautier, and M.G. Bell. Determination of uranium in urine: comparison of ICP-mass spectrometry and delayed neutron assay. Health Physics, 57:171-175 (1989).

Gladney, E.S., R.J. Peters, and D.R. Perrin. Determination of Uranium-235/Uranium-238 Natural Waters by Chelex-100 Ion Exchange and Neutron Activation. Analytical Chemistry. 55:976-977 (1983).

Gladney, E.S., D.R. Perrin, J.P. Balagna, and C.L. Warner. Evaluation of a Boron-filtered Epithermal Neutron Irradiation Facility. Analytical Chemistry. 52:2128-2132 (1980).

Gladney, E.S., W.K. Hensley, and M.M. Minor. Comparison of Three Techniques for the Measurement of Depleted Uranium in Soils. Analytical Chemistry: 50:652-653 (1978).

Gladney, E.S.; Owens, J.W.; Starner, J.W. Determination of Uranium in Natural Waters by Neutron Activation Analysis. Analytical Chemistry . 48:973-975 (1976).

Grandstaff, D.E. A kinetic study of the dissolution of uraninite. Economic Geology 71:1493-1506 (1976).

Greenberg, R.R., and H.M. Kingston. Simultaneous Determination of Twelve Trace Elements in Estuarine and Sea Water Using Pre-Irradiation Chromatography. Journal of Radioanalytical Chemistry. 71(1-2):147-167 (1982).

Greenberg, R.R., and H.M. Kingston. Trace Element Analysis of Natural Water Samples by Neutron Activation Aanalysis with Chelating Resin. Analytical Chemistry. 55:1160-1165 (1983).

Gutierrez, A.P. Physiological basis of ratio-dependent predator-prey theory: the metabolic pool model as a paradigm. Ecology 73:1552-1563 (1992).

Helton, J. C., and R.L. Inman. Sensitivity Analysis of a Model for the Environmental Movement of Radionuclides. Health Physics. 42:565-584 (1982).

Hodge, H.C. A history of uranium poisoning (1824-1942). Handbook of Experimental Pharmacology 36:5-68 (1973).

Hunter, M.D., and P.W. Price. Playing chutes and ladders: heterogeneity and the relative roles of bottomup and top-down forces in natural communities. Ecology 73:724-732 (1992).

IAEA (International Atomic Energy Agency). Effects of Ionizing Radiation on Plants and Animals Implied by Current Radiation Protection Standards. International Atomic Energy Agency Technical Report Series No. 332 (1992).

Ibrahim, S.A., and W.F. Whicker. Comparitive Uptake of $U$ and Th by Native Plants at a $U$ production Site. Health Physics. 54:413-419 (1988).

ICRP (International Commission on Radiation Protection). Recommendation of the International Commission on Radiological Protection. ICRP Publication 60. Annals of the ICRP, Vol 21, No. 1-3 (1990).

ICRP. Recommendations of the Intemational Commission on Radiological Protection: Report of the task group on reference man. ICRP Publication 26. Pergamon Press, New York, NY (1987).

Inman, R.L., and J.C. Helton. An investigation of uncertainty and sensitivity analysis techniques for computer models. Risk Analysis 8:71-90 (1988).

Inman, R.L., J.C. Helton, and J.E. Campbell. An approach to sensitivity analysis of computer models: part I introduction, input variable selection and preliminary variable assessment. Journal of Quality Technology 13:174-183 (1981a). 
Inman, R.L., J.C. Helton, and J.E. Campbell. An approach to sensitivity analysis of computer models: part II ranking of input variables, response surface validation, distribution effect and technique synopsis. Journal of Quality Technology 13:232-240 (1981b).

Jørgenson, S.E. Handbook of Environmental Data and Ecological Parameters. Pergamon Press, Ox ford, U.K. (1979).

Kingston, H.M., and R.R. Greenberg. An Elemental Rationing Technique for Assessing Concentration Data from a Complex Water System. Environment International. 10:153-161 (1984).

Kingston, H.M., I.L. Barnes, and T.C. Rains. Separation of Eight Transition Elements from Alkali and Alkaline Earth Elements in Estuarine and Seawater with Chelating Resin and their Determination by Graphite Furnace Atomic Absorption Spectrometry. Analytical Chemistry. 50:2064-2070; 1978.

Kocher, D.C. Relationship between kidney burden and radiation dose from the chronic ingestion of U: Implications for radiation standards for the public. Health Physics 57:9-15 (1989).

Kovalsky, V.V., I.E. Voronitskaya, and V.S. Lakarev. Biogeochemical food chains of uranium in aquatic and terraneous organisms. In P. Aberg and F.P. Hungate, Eds. International Symposium on Radiological Concentration Processes, Stockholm. Permagon Press (1967).

Landrum, P.F., H. Lee, II, and M.J. Lydy. Toxicokinetics in aquatic systems: model comparisons and use in hazard assessment. Environmental Toxicology and Chemistry 11:1709-1725 (1992).

Langille, B.L., E.D. Stevens, and A. Anantaraman. Cardiovascular and respiratory flow dynamics. In P.W. Webb and D. Weihs, Eds. Fish biomechanics. Prager Publ. New York, N.Y. pp. 92-139 (1983).

Langmuir, D. Uranium Solution-Mineral Equilibria at Low Temperatures with Applications to Sedimentary Ore Deposits. Geochimica et Cosmochimica Acta, 42:547-569 (1978).

Lautier, J.K., T.V. Dailey, and R.D. Brown. Effect of water restriction on feed intake of white-tailed deer. J. Wildl. Manage. 52:602-606 (1988).

Leggett, R.W. The behavior and chemical toxicity of $U$ in the kidney: a reassessment. Health Physics 57:365-383 (1989).

Li, K.C. Sliced inverse regression for dimension reduction (with discussion). Journal of the American Statistical Association 86:316-342 (1991).

Li, K.C. On principal Hessian directions for data visualization and dimension reduction: another application of Stein's lemma. Journal of the American Statistical Association 87:1025-1039 (1992).

Lindley, D.V. Introduction to probability and statistics from a Bayesian viewpoint, part 1, probability, and part 2, inference. University Press, Cambridge, MA (1965).

Lipton, J., and J.W. Gillett. Uncertainty in ocean-dumping health risks: influence of bioconcentration, commercial fish landings and seafood consumption. Environmental Toxicology and Chemistry 10:967-976 (1991).

Lovley, D.R., E.J.P. Phillips, Y.A. Gorby, and E.R. Landa. Microbial reduction of uranium. Nature 350:413-416 (1991).

Luna, L. G. Manual of histologic staining methods of the Armed Forces Institute of Pathology, 3rd edition, L. G. Luna, Ed. McGraw-Hill, New York, NY (1968).

Mackiernan, G.B. State of the Chesapeake Bay. Water Environment \& Technology 2:60-67 (1990).

Mahon, D.C. Uptake and translocation of naturally-occurring radionuclides of the uranium series. Bulletin of Environmental Contamination and Toxicology 29:697-703 (1982).

Mares, M.A. Desert rodent adaptation and community structure. Great Basin Naturalist Memoirs 7:3043 (1983). 
Matson, P., and A. Berryman. Ratio-dependent predator-prey theory. Ecology 73:1529 (1992).

Matson, P.A., and M.D. Hunter. The relative contributions of top-down and bottom-up forces in population and community ecology. Ecology 73:723 (1992).

McKay, M.D. Interim repōt on the status of research on uncertainty analysis of computer models. Los Alamos National Laboratory Report LA-UR 92-4299 (1992).

McKay, M.D., W.J. Conover, and R.J. Beckman. A comparison of three methods for selecting values of input variables in the analysis of output from a computer code. Technometrics 21:239-245 (1979).

Menge, B.A. Community regulation: under what conditions are bottom-up factors important on rocky shores? Ecology 73:755-765 (1992).

Moran, M.A., R. Benner, and R.E. Hodson. Kinetics of microbial degradation of vascular plant material in two wetland ecosystems. Oecologia 79:158-167 (1989).

Morris, M.D. Factorial sampling plans for preliminary computational experiments. Technometrics 33:161174 (1991).

Myers, O.B., P.L. Kennedy, D.J. Beckman, W.H. Clements, and M.H. Ebinger. Improving aquatic exposure assessments: uncertainty analysis of a food web exposure model. Los Alamos National Laboratory Report LA-UR 93-2004 (1993).

Nagy, K.A. Field metabolic rate and food requirement scaling in mammals and birds. Ecological Monographs 57:111-128 (1987).

NCRP (National Council on Radiation Protection and Measurements). Radiological Assessment: Predicting the Transport, Bioaccumulation, and Uptake by Man of Radionuclides Released to the Environment. National Council on Radiation Protection and Measurement Report No. 76 (1984a).

NCRP. Exposure from the uranium series with emphasis on radon and its daughters. National Council on Radiation Protection and Measurements Report 77, pp. 57-59 (1984b).

NCRP. Management of persons accidentally contaminated with radionuclides. Pages 108-112 for the National Council on Radiation Protection and Measurements Report 65, pp. 108-1 12 (1980).

Newman, M.C., and M.G. Heagler. Allometry of metal bioaccumulation and toxicity. In M.C. Newman and A.W. McIntosh, Eds. Metal toxicology: concepts and applications. Lewis Publishers, Chelsea, MI. pp. 91-130 (1991).

Nichols, D.G., and A.T. Scholz. Concentrations of Cd, Sr, and $\mathrm{U}$ in fish and water samples collected from a small stream receiving uranium mine drainage. Journal of Freshwater Ecology 5:13-25 (1989).

Norton, S.B., D.J. Rodier, J.H. Gentile, W.H. van der Schalie, W.P. Wood, and M.W. Slimak. A framework for ecological risk assessment at the EPA. Environmental Toxicology and Chemistry 11:1663-1672 (1992).

Pandian, T. J. Fish. In Animal energetics, vol. 2. Bivalvia through reptilia, T. J. Pandian and F. J. Vernberg, Eds. pp. 357-465 (1987).

Phillips, F.M., J.L. Mattick, and T.A. Duval. Chlorine 36 and Tritium from Nuclear Weapons Fallout as Tracers for Long-Term Liquid and Vapor Movement in Desert Soils. Water Resources Research. 24:1877-1891 (1988).

Polis, G.A., Editor. The ecology of desert communities. The University of Arizona Press, Tucson, AZ (1991).

Poston, T.M. Observations on the bioaccumulation potential of thorium and uranium in rainbow trout (Salmo gairdneri). Bulletin of Environmental Contamination and Toxicology 28:682-690 (1982).

Poston, T.M. Observations on the bioaccumulation potential of thorium and uranium in rainbow trout (Salmo gairdneri). Bulletin of Environmental Contamination and Toxicology 28:682-690 (1983). 
Power, M.E. Top-down and bottom-up forces in food webs: do plants have primacy? Ecology 73:733746 (1992).

Price, K.R. The analysis of soil and vegetation samples collected from the Yuma Proving Ground. Pacific Northwest Laboratory Report TD 2761 (1991).

Ronen, Y. Uncertainty Analysis. CRC Press, Boca Raton, FL (1988).

Savage, L.J. The Foundations of Statistics. Wiley and Sons, New York, NY (1954).

Scanlon, B.R., P.W. Kubik, P. Sharma, B.C. Richter, and H.E. Gove. Bomb Chlorine-36 Analysis in the Characterization of Unsaturated Flow at a Proposed Radioactive Waste Disposal Facility, Chihuahuan Desert, Texas. Nuclear Instruments and Methods in Physics Research. B52:489492 (1990).

Scientific Ecology Group. Jefferson Proving Ground Depleted Uranium Impact Area Scoping Survey Report. Scientific Ecology Group, Oak Ridge, TN (1995).

Slobodkin, L.B. A summary of the special feature and comments on its theoretical context and importance. Ecology 73:1564-1566 (1992).

Strong, D.R. Are trophic levels all wet? Differentiation and donor-control in speciose ecosystems. Ecology 73:747-754 (1992).

SuLu, S., and F. Zhao. Nephrotoxic limit and annual limit on intake for natural U. Health Physics 58:619-623 (1990).

Thompson, C.E., C.A. Burton, D.J. Quinn, and Y.C. Ng. Concentration factors of chemical elements in edible aquatic organisms. University of California Radiation Laboratory Report 50564 Rev. 1 (1972).

Trabalka, J.R., and J.T. Garten, Jr. Behavior of the long- lived synthetic elements and their natural analogs in food chains. Advances in Radiation Biology 10:39-104 (1983).

U.S. Army (U.S. Army Combat Systems Test Activity). Environmental assessment for testing of depleted uranium penetrator munitions. U.S. Army Combat Systems Test Activity Report (1990a).

U.S. Army. Hydrological Investigation, Environmental Reconnaissance Sampling Wells. Report prepared by U. S. Army Engineer District, Baltimore, Engineer Division, Geotechnical Engineering Branch, Army Corps of Engineers (1990b).

U.S.D.A. (U.S. Department of Agriculture). Soil Survey of Harford County Area, Maryland. USDA Soil Conservation Service with Maryland Agricultural Experiment Station (1975).

Van Straalen, N.M., T.B.A. Burghouts, M.J. Doornhof, G.M. Groot, M.P.M. Janssen, E.N.G. Joosse, J.H. van Meerendonk, J.P.J.J. Theeuwen, H.A. Verhoef, and H.R. Zoomer. Efficiency of lead and cadmium excretions in populations of Orchesella cincta (Collembola) from various contaminated forest soils. Journal of Applied Ecology 24:953-968 (1987).

Walker, F.W., G.J. Kirouac, and F.M. Rourke. Chart of the nuclides, 12th ed. General Electric Company, San Jose, CA (1977).

Walne, P.R. The influence of current speed, body size, and water temperature on the filtration rate of five species of bivalves. J. Marine Biol. Assoc., U.K. 52:345-374 (1972).

Ward, T.J., and K.A.Stevens. Modeling Erosion and Transport of Depeleted Uranium, Yuma Proving Ground, Arizona. New Mexico Water Resources Research Institute (WRRI) Report No. 286, New Mexico State University, Las Cruces, NM (1994).

Whicker, F.W., and T.B. Kirchner. PATHWAY: a dynamic food-chain model to predict radionuclide ingestion after fallout deposition. Health Physics 52:717-737 (1987).

Wrenn, M.E., P.W. Durbin, D.L. Willis, and N. Singh. The Potential Toxicity of Uranium in Water. J. Am. Water Works Assoc. 79:177-184 (1987). 
Wrenn, M.E., P.W. Durbin, B. Howard, J. Lipsztein, J. Rundo, E.T. Still, and D.L. Willis. Metabolism of ingested U and Ra. Health Physics 48:601-633 (1985).

Yu, C., A.J.Zeilen, J.-J. Cheng, Y.C. Yuan, L.G. Jones, D.J. Lepoire, Y.Y. Wang, C.O. Loureiro, E. Gnanapragasam, E. Faillace, A. Wallo, W.A. Williams, and H. Peterson. Manual for Implementing Residual Radioactive Material Guidelines Using RESRAD 5.0. Argonne National Laboratory Report ANL/EAD/LD-2 (1993).

Yuile, C.L. Animal experiments. Handbook of Experimental Pharmacology 36:165-196 (1973).

Zach, R., and K. R. Mayoh. Soil ingestion by cattle: a neglected pathway. Health Physics 46:426-431 (1984).

Zhao, F-Y. and S-L. Zhao. Nephrotoxic Limit and Annual Limit on Intake for Natural U. Health Physics. 58:619-623 (1990). 


\section{Appendix A}

Parameter Values and Ranges Used in APG Aquatic Model 
Table A-1. Parameter values for APG aquatic model.

\begin{tabular}{|c|c|c|c|c|c|c|}
\hline Parameter & Nominal value & Range & Units & Category & Explanation & Source \\
\hline $\mathrm{b}_{1}$ & 0.0100 & $0.0079-0.0138$ & $\mathrm{~kg} / \mathrm{m}^{2}$ & Structural & dry mass as phytoplankton & Baird and Ulanowicz 1989 \\
\hline$b_{2}$ & 0.0800 & $0.0764-0.0939$ & $\mathrm{~kg} / \mathrm{m}^{2}$ & Structural & $\begin{array}{l}\text { dry mass as suspended } \\
\text { organic matter (OM) }\end{array}$ & Baird and Ulanowicz 1989 \\
\hline$b_{3}$ & 0.9000 & $0.802-1.020$ & $\mathrm{~kg} / \mathrm{m}^{2}$ & Structural & dry mass as sediment $O M$ & Baird and Ulanowicz 1989 \\
\hline$b_{4}$ & 0.0005 & $0.0001-0.0010$ & $\mathrm{~kg} / \mathrm{m}^{2}$ & Structural & $\begin{array}{c}\text { dry mass as } \\
\text { benthic diatoms }\end{array}$ & Baird and Ulanowicz 1989 \\
\hline $\mathbf{b}_{5}$ & 0.0020 & $0.0012-0.0035$ & $\mathrm{~kg} / \mathrm{m}^{2}$ & Structural & dry mass as zooplankton & Baird and Ulanowicz 1989 \\
\hline $\mathbf{b}_{6}$ & 0.0030 & $0.0025-0.0050$ & $\mathrm{~kg} / \mathrm{m}^{2}$ & Structural & $\begin{array}{l}\text { dry mass as benthic } \\
\text { suspension feeders }\end{array}$ & Baird and Ulanowicz 1989 \\
\hline$b_{7}$ & 0.0090 & $0.0048-0.0123$ & $\mathrm{~kg} / \mathrm{m}^{2}$ & Structural & $\begin{array}{l}\text { dry mass as benthic } \\
\text { deposit feeders }\end{array}$ & Baird and Ulanowicz 1989 \\
\hline$b_{8}$ & 0.0002 & $0.00008-0.00028$ & $\mathrm{~kg} / \mathrm{m}^{2}$ & Structural & $\begin{array}{l}\text { dry mass as suspension } \\
\text { feeding fish }\end{array}$ & Baird and Ulanowicz 1989 \\
\hline$b_{9}$ & 0.0002 & $0.00004-0.00040$ & $\mathrm{~kg} / \mathrm{m}^{2}$ & Structural & $\begin{array}{l}\text { dry mass as } \\
\text { carnivorous fish }\end{array}$ & Baird and Ulanowicz 1989 \\
\hline$b_{10}$ & 75 & $37.5-112.5$ & $\mathrm{~kg} / \mathrm{m}^{2}$ & Structural & $\begin{array}{l}\text { dry mass as } \\
\text { labile sediment }\end{array}$ & Baird and Ulanowicz 1989 \\
\hline$B C F_{1}$ & 880 & $16-1700$ & dimen-sionless & $\begin{array}{l}\text { Bioconcentra- } \\
\text { tion factor }\end{array}$ & $\begin{array}{l}\text { calculates phytoplankton } \\
\text { concentration from water } \\
\text { concentration }\end{array}$ & $\begin{array}{l}\text { Langille et al. 1983; } \\
\text { Jørgenson } 1979\end{array}$ \\
\hline$B C F_{2}$ & 39 & $2-280$ & dimen-sionless & $\begin{array}{l}\text { Bioconcentra- } \\
\text { tion factor }\end{array}$ & $\begin{array}{l}\text { calculates suspended } \mathrm{OM} \\
\text { concentration from water } \\
\text { concentration }\end{array}$ & $\begin{array}{l}\text { Langille et al. 1983; } \\
\text { Jørgenson } 1979\end{array}$ \\
\hline$B C F_{4}$ & 880 & $16-1700$ & dimen-sionless & $\begin{array}{l}\text { Bioconcentra- } \\
\text { tion factor }\end{array}$ & $\begin{array}{l}\text { calculates benthic diatom } \\
\text { concentration from } \\
\text { waterconcentration }\end{array}$ & $\begin{array}{l}\text { Langille et al. 1983; } \\
\text { Jørgenson } 1979\end{array}$ \\
\hline$C_{0}$ & 0.003 & $0.0003-3$ & $\mathrm{mg}-\mathrm{DU} / \mathrm{kg}$ & Source & water concentration & Moran et al. 1989 \\
\hline$c_{1}$ & 0 & - & $\mathrm{mg}-\mathrm{DU} / \mathrm{kg}$ & State variable & $\begin{array}{l}\text { phytoplankton } \\
\text { concentration }\end{array}$ & - \\
\hline$C_{2}$ & 0 & - & $\mathrm{mg}-\mathrm{DU} / \mathrm{kg}$ & State variable & $\begin{array}{l}\text { suspended OM } \\
\text { concentration }\end{array}$ & - \\
\hline$C_{3}$ & 0 & - & $\mathrm{mg}-\mathrm{DU} / \mathrm{kg}$ & State variable & $\begin{array}{l}\text { sediment } \mathrm{OM} \\
\text { concentration }\end{array}$ & - \\
\hline$C_{4}$ & 0 & - & $\mathrm{mg}-\mathrm{DU} / \mathrm{kg}$ & State variable & $\begin{array}{l}\text { benthic diatoms } \\
\text { concentration }\end{array}$ & - \\
\hline$C_{5}$ & 0 & - & $\mathrm{mg}-\mathrm{DU} / \mathrm{kg}$ & State variable & $\begin{array}{l}\text { zooplankton } \\
\text { concentration }\end{array}$ & - \\
\hline$C_{6}$ & 0 & - & $\mathrm{mg}-\mathrm{DU} / \mathrm{kg}$ & State variable & $\begin{array}{l}\text { benthic suspension } \\
\text { feeders concentration }\end{array}$ & - \\
\hline$C_{7}$ & 0 & - & $\mathrm{mg}-\mathrm{DU} / \mathrm{kg}$ & State variable & $\begin{array}{l}\text { benthic deposit } \\
\text { feeders concentration }\end{array}$ & - \\
\hline$C_{8}$ & 0 & - & $\mathrm{mg}-\mathrm{DU} / \mathrm{kg}$ & State variable & $\begin{array}{l}\text { suspension feeding fish } \\
\text { concentration }\end{array}$ & - \\
\hline
\end{tabular}


Table A-1. (cont.)

\begin{tabular}{|c|c|c|c|c|c|c|}
\hline Parameter & Nominal value & Range & Units & Category & Explanation & Source \\
\hline$C_{9}$ & 0 & - & $\mathrm{mg}-\mathrm{DU} / \mathrm{kg}$ & State variable & $\begin{array}{l}\text { camivorous fish } \\
\text { concentration }\end{array}$ & - \\
\hline$c_{10}$ & 0 & - & $\mathrm{mg}-\mathrm{DU} / \mathrm{kg}$ & State variable & $\begin{array}{l}\text { labile sediment } \\
\text { concentration }\end{array}$ & - \\
\hline$d_{7}$ & 0.4 & $0.3-0.5$ & $\begin{array}{l}\text { kg-dry matter } \\
(D M) / k g-w e t\end{array}$ & Physiological & converts fresh mass to DM & Trabalka and Garten 1983 \\
\hline $\mathrm{d}_{8}$ & 0.4 & $0.3-0.5$ & $\mathrm{~kg}$-DM/kg-wet & Physiological & converts fresh mass to $D M$ & Trabalka and Garten 1983 \\
\hline$d_{g}$ & 0.4 & $0.3-0.5$ & $\mathrm{~kg}-\mathrm{DM} / \mathrm{kg}$-wet & Physiological & converts fresh mass to DM & Trabalka and Garten 1983 \\
\hline$a_{5,0}$ & 0.01 & $0.0003-0.08$ & dimen-sionless & Physiological & assimilation efficiency & Wrenn et al. 1985 \\
\hline$a_{5,1}$ & 0.01 & $0.0003-0.08$ & dimen-sionless & Physiological & assimilation efficiency & Wrenn et al. 1985 \\
\hline$a_{5,2}$ & 0.01 & $0.0003-0.08$ & dimen-sionless & Physiological & assimilation efficiency & Wrenn et al. 1985 \\
\hline$a_{B, 0}$ & 0.01 & $0.0003-0.08$ & dimen-sionless & Physiological & assimilation efficiency & Wrenn etal. 1985 \\
\hline$a_{6,1}$ & 0.01 & $0.0003-0.08$ & dimen-sionless & Physiological & assimilation efficiency & Wrenn et al. 1985 \\
\hline$a_{6,3}$ & 0.01 & $0.0003-0.08$ & dimen-sionless & Physiological & assimilation efficiency & Wrenn et al. 1985 \\
\hline$a_{6,5}$ & 0.01 & $0.0003-0.08$ & dimen-sionless & Physiological & assimilation efficiency & Wrenn et al. 1985 \\
\hline$a_{7,0}$ & 0.01 & $0.0003-0.08$ & dimen-sionless & Physiological & assimilation efficiency & Wrenn et al. 1985 \\
\hline$a_{7,3}$ & 0.01 & $0.0003-0.08$ & dimen-sionless & Physiological & assimilation efficiency & Wrenn etal. 1985 \\
\hline$a_{7,4}$ & 0.01 & $0.0003-0.08$ & dimen-sionless & Physiological & assimilation efficiency & Wrenn etal. 1985 \\
\hline$a_{7,6}$ & 0.01 & $0.0003-0.08$ & dimen-sionless & Physiological & assimilation efficiency & Wrenn et al. 1985 \\
\hline$a_{8,0}$ & 0.01 & $0.0003-0.08$ & dimen-sionless & Physiological & assimilation efficiency & Wrenn etal. 1985 \\
\hline$a_{8,1}$ & 0.01 & $0.0003-0.08$ & dimen-sionless & Physiological & assimilation efficiency & Wrenn et al. 1985 \\
\hline$a_{8,5}$ & 0.01 & $0.0003-0.08$ & dimen-sionless & Physiological & assimilation efficiency & Wrenn etal. 1985 \\
\hline$a_{9,0}$ & 0.01 & $0.0003-0.08$ & dimen-sionless & Physiological & assimilation efficiency & Wrenn etal. 1985 \\
\hline$a_{9,6}$ & 0.01 & $0.0003-0.08$ & dimen-sionless & Physiological & assimilation efficiency & Wrenn etal. 1985 \\
\hline$a_{9,7}$ & 0.01 & $0.0003-0.08$ & dimen-sionless & Physiological & assimilation efficiency & Wrenn et al. 1985 \\
\hline$a_{9,8}$ & 0.01 & $0.0003-0.08$ & dimen-sionless & Physiological & assimilation efficiency & Wrenn et al. 1985 \\
\hline$F_{10,3}$ & 0.5 & $0-1$ & dimen-sionless & Abiotic & $\begin{array}{c}\text { fraction of } C_{3} \text { released to } \\
\text { sediment }\end{array}$ & - \\
\hline$I_{10,0}$ & 3.11 & $1.55-4.66$ & per day & Abiotic & water to sediment transfer & $\begin{array}{l}\text { Calder 1984; Newman } \\
\text { and Heagler } 1991\end{array}$ \\
\hline$l_{10,3}$ & 0.011 & $0.001-0.02$ & per day & Abiotic & decomposition rate & $\begin{array}{c}\text { Trabalka and Garten 1983; } \\
\text { Benninger et al. } 1979\end{array}$ \\
\hline$k_{11,5}$ & 0.0617 & $\pm 50 \%$ & per day & Functional & mass transfer rate & Norton etal. 1992 \\
\hline$k_{11,6}$ & 0.0061 & $\pm 50 \%$ & per day & Functional & mass transfer rate & Norton et al. 1992 \\
\hline$k_{11,7}$ & 0.0011 & $\pm 50 \%$ & per day & Functional & mass transfer rate & Norton et al. 1992 \\
\hline$k_{11,8}$ & 0.0039 & $\pm 50 \%$ & per day & Functional & mass transfer rate & Norton et al. 1992 \\
\hline$k_{11,9}$ & 0.0039 & $\pm 50 \%$ & per day & Functional & mass transfer rate & Norton etal. 1992 \\
\hline
\end{tabular}


Table A-1. (cont.)

\begin{tabular}{|c|c|c|c|c|c|c|}
\hline Parameter & Nominal value & Range & Units & Category & Explanation & Source \\
\hline$I_{12,10}$ & 0.0001 & $4.6 E-05-1.0 E-04$ & perday & Abiotic & sedimentation rate & Bowie et al. 1985 \\
\hline$k_{2,1}$ & 0.147 & $\pm 50 \%$ & per day & Functional & mass transfer rate & Norton et al. 1992 \\
\hline$k_{2,5}$ & 0.296 & $\pm 50 \%$ & per day & Functional & mass transfer rate & Norton et al. 1992 \\
\hline$k_{3,2}$ & 0.0269 & $\pm 50 \%$ & per day & Functional & mass transfer rate & Norton et al. 1992 \\
\hline$k_{3,5}$ & 0.0027 & $\pm 50 \%$ & per day & Functional & mass transfer rate & Norton et al. 1992 \\
\hline$k_{3,6}$ & 0.011 & $\pm 50 \%$ & perday & Functional & mass transfer rate & Norton et al. 1992 \\
\hline$k_{3,7}$ & 0.149 & $\pm 50 \%$ & per day & Functional & mass transfer rate & Norton et al. 1992 \\
\hline$k_{3,8}$ & 0.0338 & $\pm 50 \%$ & per day & Functional & mass transfer rate & Norton et al. 1992 \\
\hline$k_{3,9}$ & 0.0145 & $\pm 50 \%$ & per day & Functional & mass transfer rate & Norton etal. 1992 \\
\hline$k_{5,0}$ & 35 & $2.28-79.8$ & $L / m^{2}-$ day $^{-1}$ & Physiological & ventilation rate & $\begin{array}{c}\text { Trabalka and Garten 1983; } \\
\text { Walne } 1972\end{array}$ \\
\hline$k_{5,1}$ & 0.0542 & $\pm 50 \%$ & per day & Functional & mass transfer rate & Norton et al. 1992 \\
\hline$k_{5,2}$ & 0.023 & $\pm 50 \%$ & per day & Functional & mass transfer rate & Norton et al. 1992 \\
\hline$k_{6,0}$ & 0.44 & $0.116-0.756$ & $\mathrm{~L} / \mathrm{m}^{2}-\mathrm{day}^{-1}$ & Physiological & ventilation rate & Pandian 1987 \\
\hline$k_{6.1}$ & 0.0086 & $\pm 50 \%$ & per day & Functional & mass transfer rate & Norton et al. 1992 \\
\hline$k_{6,2}$ & 0.0007 & $\pm 50 \%$ & per day & Functional & mass transfer rate & Norton et al. 1992 \\
\hline$k_{6,5}$ & 0.0026 & $\pm 50 \%$ & per day & Functional & mass transfer rate & Norton et al. 1992 \\
\hline$k_{7,3}$ & 0.0027 & $\pm 50 \%$ & per day & Functional & mass transfer rate & Norton et al. 1992 \\
\hline$k_{7,4}$ & 0.28 & $\pm 50 \%$ & per day & Functional & mass transfer rate & Norton et al. 1992 \\
\hline$k_{7,6}$ & 0.0013 & $\pm 50 \%$ & perday & Functional & mass transfer rate & Norton et al. 1992 \\
\hline$k_{8,1}$ & 0.0002 & $\pm 50 \%$ & per day & Functional & mass transfer rate & Norton et al. 1992 \\
\hline$k_{8,2}$ & 4E-05 & $\pm 50 \%$ & per day & Functional & mass transfer rate & Norton et al. 1992 \\
\hline$k_{8,5}$ & 0.0062 & $\pm 50 \%$ & perday & Functional & mass transfer rate & Norton et al. 1992 \\
\hline$k_{9,6}$ & $2 \mathrm{E}-05$ & $\pm 50 \%$ & per day & Functional & mass transfer rate & Norton et al. 1992 \\
\hline$k_{9,7}$ & 0.0006 & $\pm 50 \%$ & per day & Functional & mass transfer rate & Norton et al. 1992 \\
\hline$k_{9,8}$ & 0.0045 & $\pm 50 \%$ & per day & Functional & mass transfer rate & Norton etal. 1992 \\
\hline$g_{7}$ & 25 & $1-5$ & dimen-sionless & Physiological & metabolic rate multiplier & - \\
\hline $\mathrm{g}_{8}$ & 25 & $1-5$ & dimen-sionless & Physiological & metabolic rate multiplier & - \\
\hline$g_{9}$ & 25 & $1-5$ & dimen-sionless & Physiological & metabolic rate multiplier & - \\
\hline$Q_{11}$ & 0 & - & $\mathrm{mg}-\mathrm{DU} / \mathrm{m}^{2}$ & State variable & quantity DU exported & - \\
\hline$Q_{12}$ & 0 & - & $\mathrm{mg}-\mathrm{DU} / \mathrm{m}^{2}$ & State variable & $\begin{array}{l}\text { quantity DU in deep } \\
\text { sediment sink }\end{array}$ & - \\
\hline$r_{5}$ & 0.718 & $\pm 50 \%$ & per day & Physiological & biomass tumover rate & Norton et al. 1992 \\
\hline$r_{6}$ & 0.014 & $\pm 50 \%$ & per day & Physiological & biomass tumover rate & Norton et al. 1992 \\
\hline$r_{7}$ & 0.06 & $\pm 50 \%$ & per day & Physiological & biomass turnover rate & Norton et al. 1992 \\
\hline
\end{tabular}


Table A-1. (cont.)

\begin{tabular}{|c|c|c|c|c|c|c|}
\hline Parameter & Nominal value & Range & Units & Category & Explanation & Source \\
\hline $\mathbf{r}_{8}$ & 0.025 & $\pm 50 \%$ & per day & Physiological & biomass tumover rate & Norton et al. 1992 \\
\hline$r_{g}$ & 0.009 & $\pm 50 \%$ & perday & Physiological & biomass tumover rate & Norton et al. 1992 \\
\hline$n_{7}$ & 235 & $222-445$ & L/kg-day & Physiological & $\begin{array}{c}\text { standard } \\
\text { ventilation volume }\end{array}$ & Mahon 1982 \\
\hline $\mathrm{n}_{8}$ & 235 & $222-445$ & L/kg-day & Physiological & $\begin{array}{c}\text { standard } \\
\text { ventilation volume }\end{array}$ & Mahon 1982 \\
\hline $\mathrm{n}_{\mathbf{g}}$ & 235 & $222-445$ & L/kg-day & Physiological & $\begin{array}{c}\text { standard } \\
\text { ventilation volume }\end{array}$ & Mahon 1982 \\
\hline$t_{5}$ & 1.1 & $0.33-10$ & dimen-sionless & Physiological & respiration rate multiplier & - \\
\hline$t_{6}$ & 1.1 & $0.33-10$ & dimen-sionless & Physiological & respiration rate multiplier & - \\
\hline$t_{7}$ & 1.1 & $0.33-10$ & dimen-sionless & Physiological & respiration rate multiplier & - \\
\hline$t_{8}$ & 1.1 & $0.33-10$ & dimen-sionless & Physiological & respiration rate multiplier & - \\
\hline$t_{9}$ & 0.1 & $0.33-10$ & dimen-sionless & Physiological & respiration rate multiplier & - \\
\hline
\end{tabular}




\section{Appendix B}

Uncertainty and Sensitivity Analyses for the Steady State Model, APG Deer

B-1 
Uncertainty and Sensitivity Analyses for the Steady-State Model

\section{Sheet: DMOD4A.XLS}

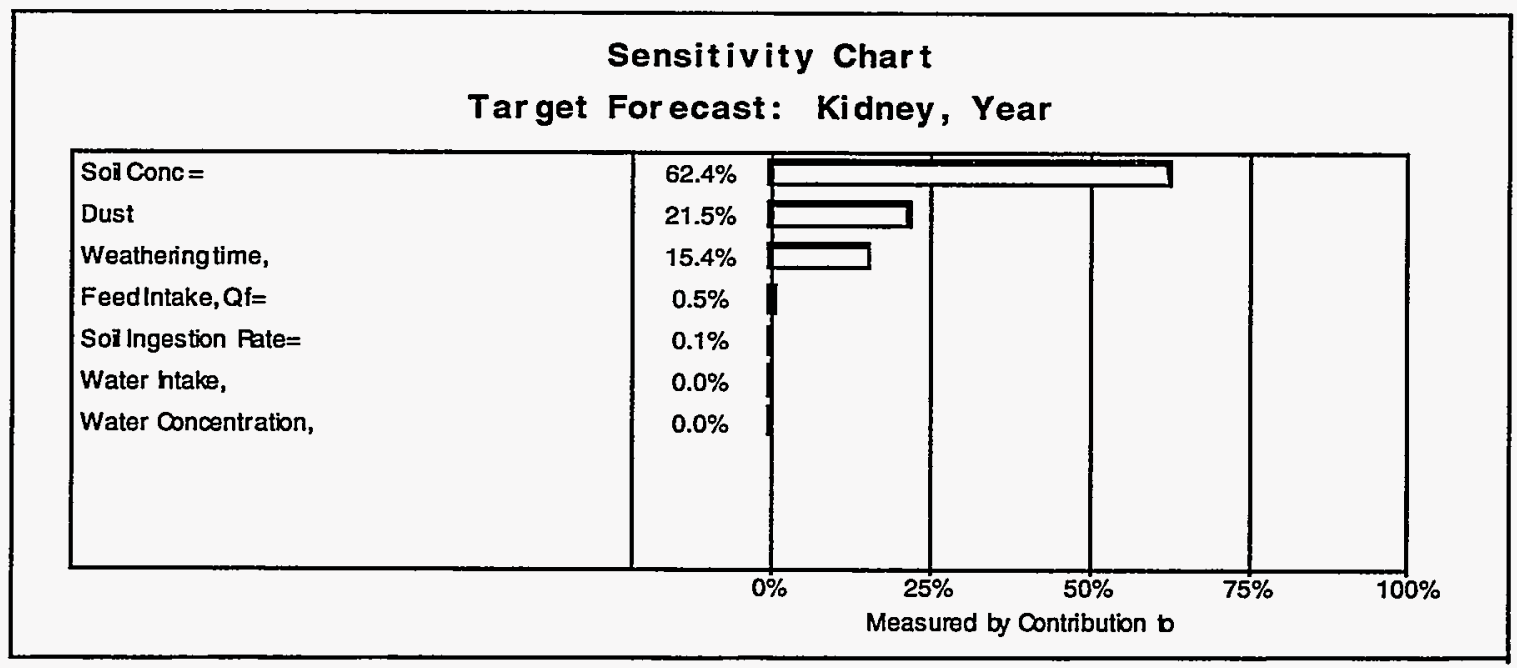


Summary:

Certainty Level is $9.50 \mathrm{E}+1 \%$

Certainty Range is from $2.53 \mathrm{E}-12$ to $1.56 \mathrm{E}-9$ Detriments

Display Range is from $0.00 E+0$ to $2.00 E-9$ Detriments

Entire Range is from $7.43 \mathrm{E}-13$ to $9.95 \mathrm{E}-9$ Detriments

After 10,000 Trials, the Std. Error of the Mean is 6.67E-12

Statistics:

Trials

Mean

Median (approx.)

Mode (approx.)

Standard Deviation

Variance

Skewness

Kurtosis

Coeff. of Variability

Range Minimum

Range Maximum

Range Width

Mean Std. Error
Value

10000

2.13E-10

$5.86 \mathrm{E}-11$

5.05E-11

6.67E-10

4.45E-19

$7.40 \mathrm{E}+00$

$6.81 \mathrm{E}+01$

3.14E+00

7.43E-13

9.95E-09

9.94E-09

6.67E-12

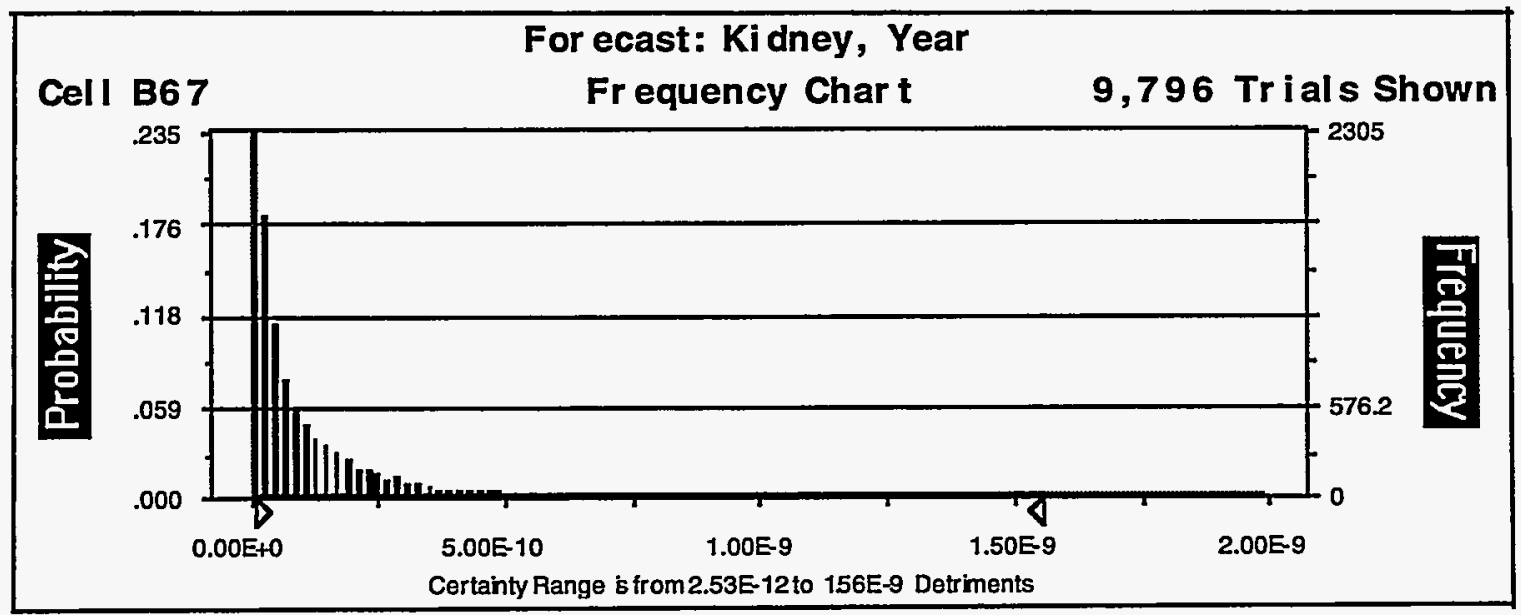




\section{Summary:}

Certainty Level is $9.50 \mathrm{E}+1 \%$

Certainty Range is from 1.26E-10 to $7.80 \mathrm{E}-8$ Detriments

Display Range is from $0.00 \mathrm{E}+0$ to $1.00 \mathrm{E}-7$ Detriments

Entire Range is from 3.72E-11 to 4.97E-7 Detriments

After 10,000 Trials, the Std. Error of the Mean is 3.33E-10

Statistics:

Trials

Mean

Median (approx.)

Mode (approx.)

Standard Deviation

Variance

Skewness

Kurtosis

Coeff. of Variability

Range Minimum

Range Maximum

Range Width

Mean Std. Error
Value

10000

1.06E-08

2.93E-09

2.52E-09

3.33E-08

$1.11 \mathrm{E}-15$

$7.40 \mathrm{E}+00$

$6.81 E+01$

$3.14 \mathrm{E}+00$

3.72E-11

4.97E-07

4.97E-07

3.33E-10

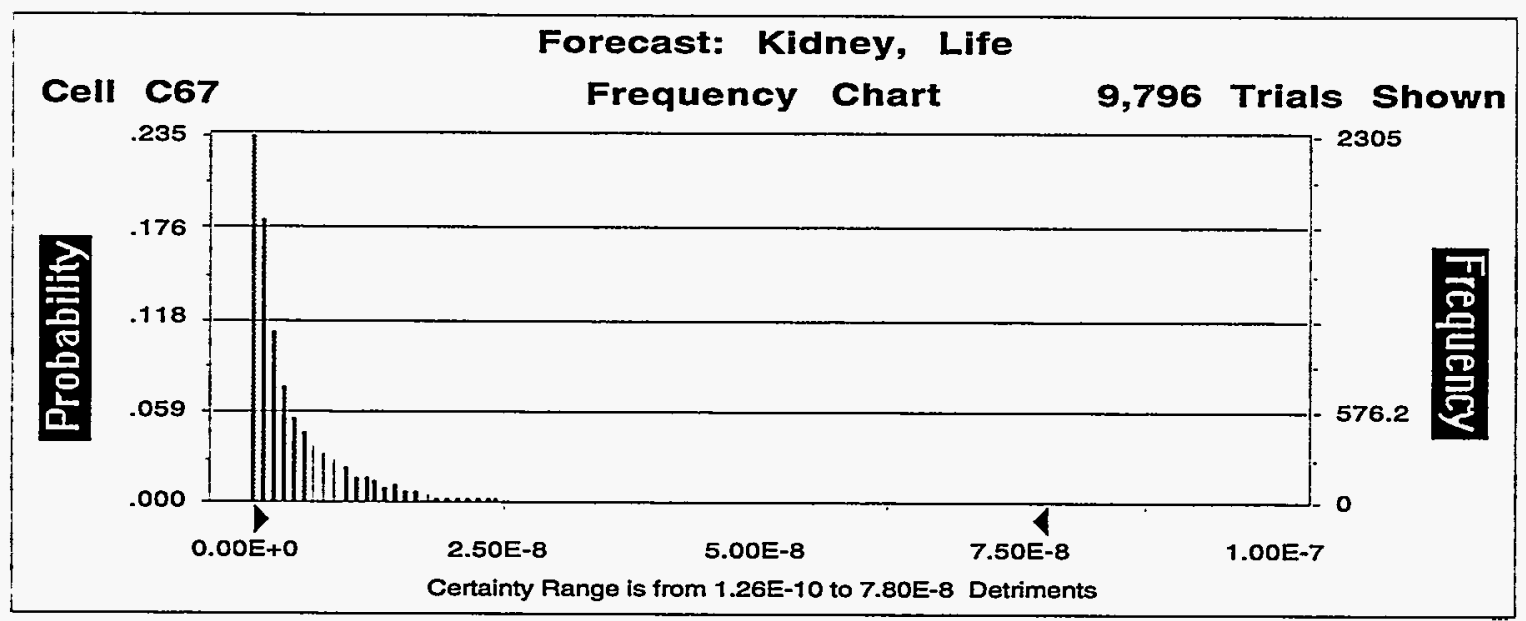


Summary:

Certainty Level is $9.50 \mathrm{E}+1 \%$

Certainty Range is from $1.24 \mathrm{E}-12$ to $7.70 \mathrm{E}-10$ Detriments

Display Range is from $0.00 \mathrm{E}+0$ to $1.00 \mathrm{E}-9$ Detriments

Entire Range is from 3.67E-13 to 4.91E-9 Detriments

After 10,000 Trials, the Std. Error of the Mean is $3.29 \mathrm{E}-12$

Statistics:

Trials

Mean

Median (approx.)

Mode (approx.)

Standard Deviation

Variance

Skewness

Kurtosis

Coeff. of Variability

Range Minimum

Range Maximum

Range Width

Mean Std. Error
Value

10000

1.05E-10

2.89E-11

2.49E-11

3.29E-10

1.08E-19

$7.40 E+00$

$6.81 E+01$

3. $14 \mathrm{E}+00$

3.67E-13

4.91E-09

4.91E-09

3.29E-12

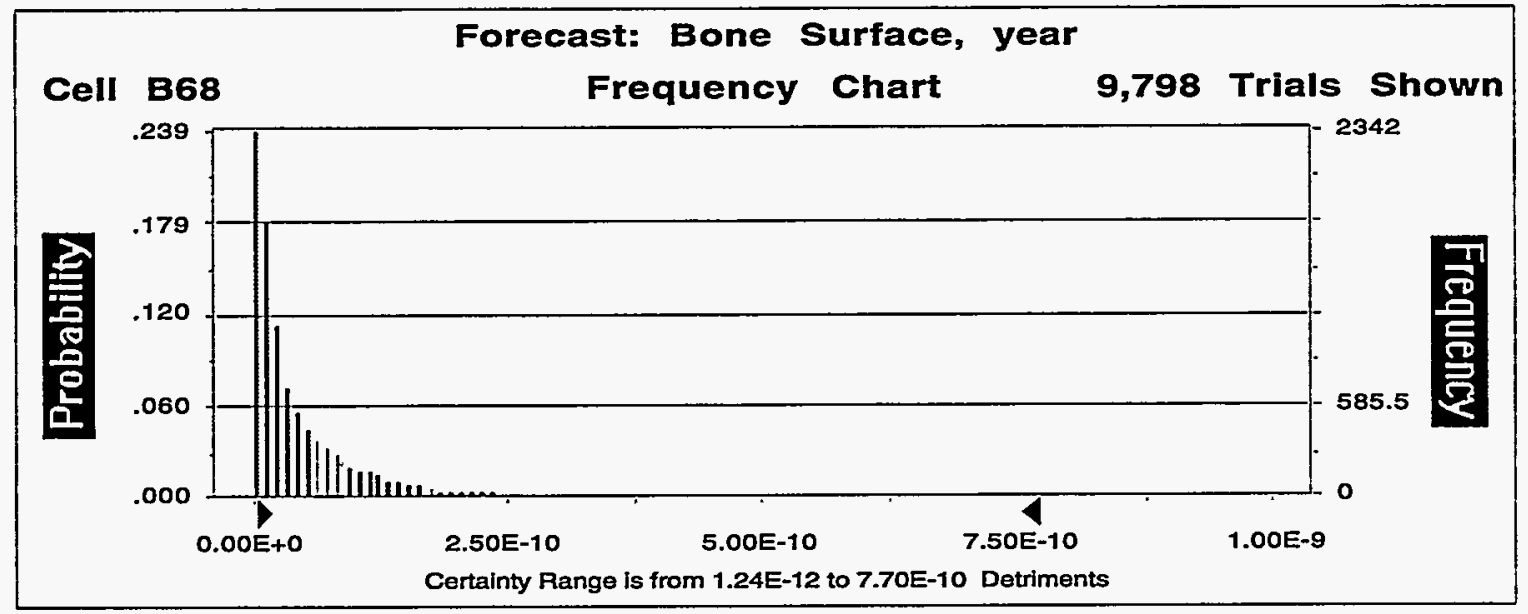


Summary:

Certainty Level is $9.50 \mathrm{E}+1 \%$

Certainty Range is from $6.22 \mathrm{E}-11$ to $3.85 \mathrm{E}-8$ Detriments

Display Range is from $0.00 \mathrm{E}+0$ to $5.00 \mathrm{E}-8$ Detriments

Entire Range is from 1.83E-11 to 2.45E-7 Detriments

After 10,000 Trials, the Std. Error of the Mean is $1.65 \mathrm{E}-10$

Statistics:

Trials

Mean

Median (approx.)

Mode (approx.)

Standard Deviation

Variance

Skewness

Kurtosis

Coeff. of Variability

Range Minimum

Range Maximum

Range Width

Mean Std. Error
Value

10000

5.25E-09

1.45E-09

1.24E-09

1.65E-08

2.71E-16

$7.40 E+00$

$6.81 E+01$

$3.14 \mathrm{E}+00$

1.83E-11

2.45E-07

2.45E-07

1.65E-10

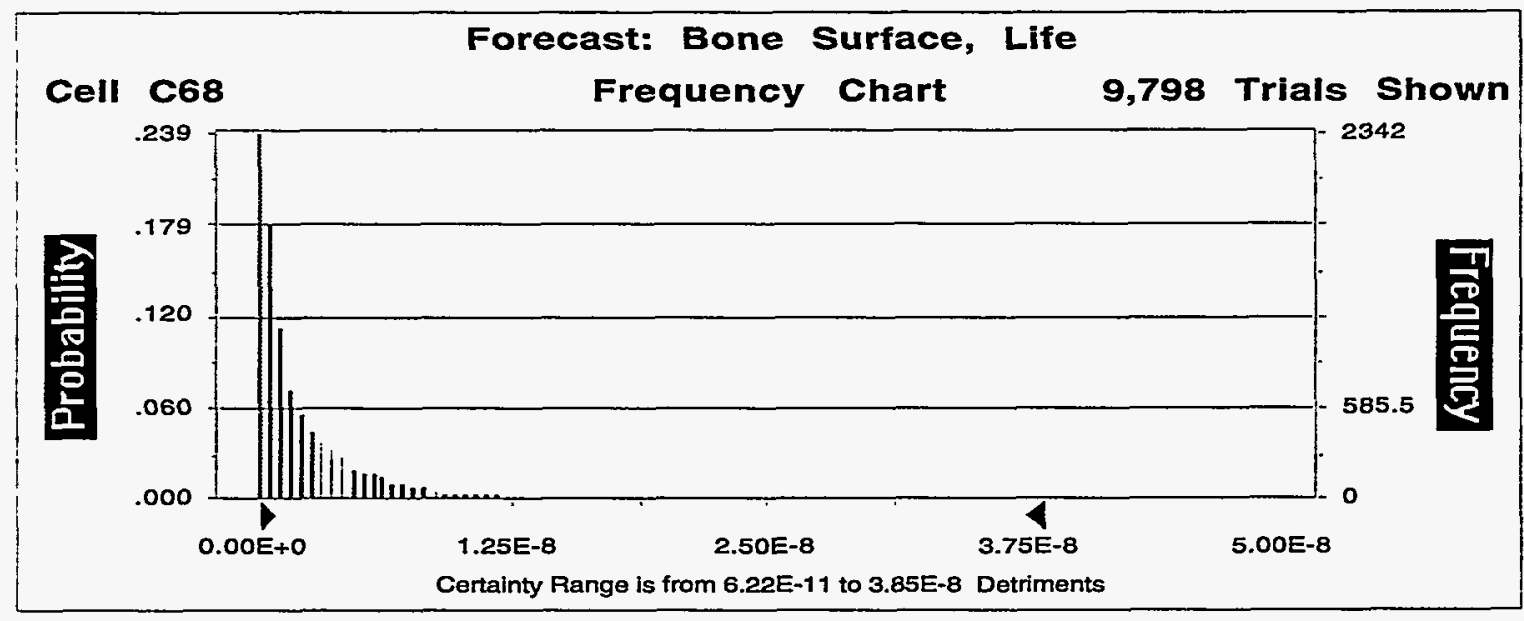


Summary:

Certainty Level is $9.50 \mathrm{E}+1 \%$

Certainty Range is from $8.36 \mathrm{E}-14$ to $5.20 \mathrm{E}-11$ Detriments

Display Range is from $0.00 \mathrm{E}+0$ to $7.00 \mathrm{E}-11$ Detriments

Entire Range is from 2.48E-14 to $3.32 \mathrm{E}-10$ Detriments

After 10,000 Trials, the Std. Error of the Mean is 2.22E-13

Statistics:

Trials

Mean

Median (approx.)

Mode (approx.)

Standard Deviation

Variance

Skewness

Kurtosis

Coeff. of Variability

Range Minimum

Range Maximum

Range Width

Mean Std. Error
Value

10000

7.09E-12

1.95E-12

1.68E-12

2.22E-11

4.94E-22

$7.40 E+00$

$6.81 E+01$

$3.14 E+00$

2.48E-14

3.32E-10

3.31E-10

2.22E-13

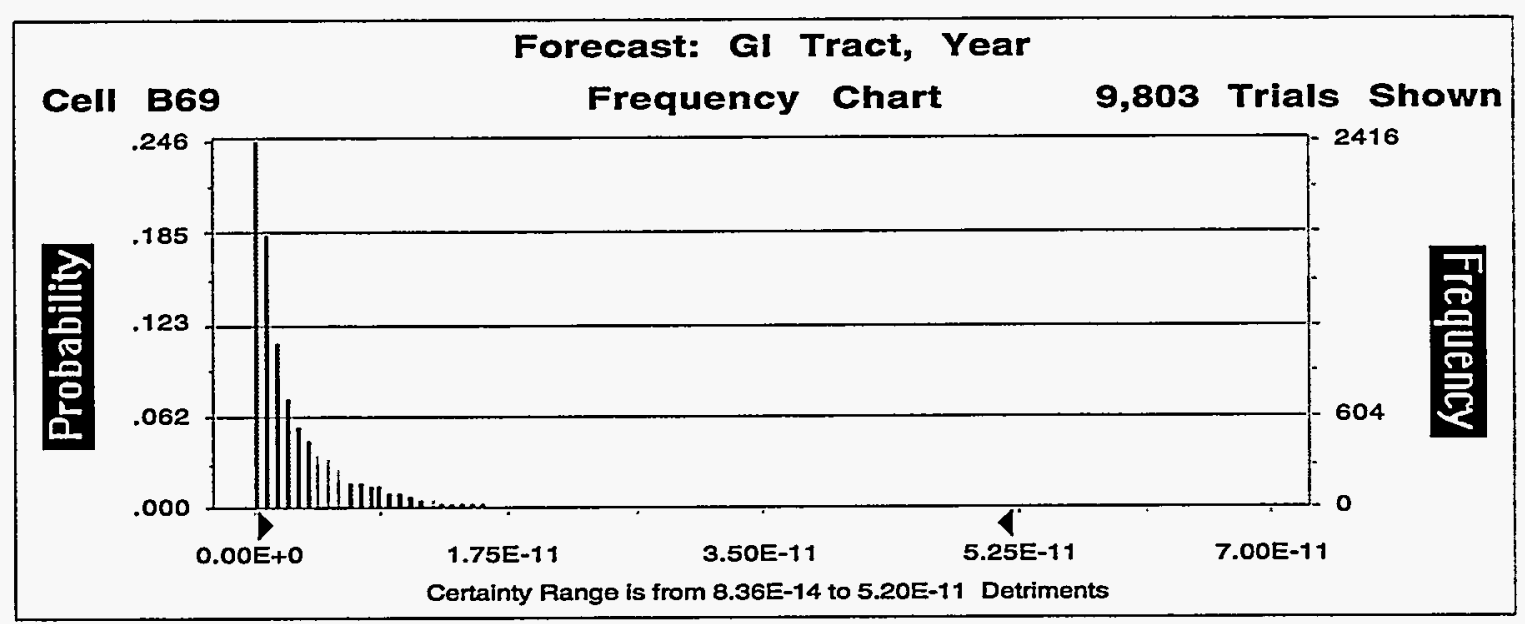


Summary:

Certainty Level is $9.50 \mathrm{E}+1 \%$

Certainty Range is from $4.18 \mathrm{E}-12$ to $2.60 \mathrm{E}-9$ Detriments

Display Range is from $0.00 \mathrm{E}+0$ to $3.50 \mathrm{E}-9$ Detriments

Entire Range is from 1.24E-12 to $1.66 \mathrm{E}-8$ Detriments

After 10,000 Trials, the Std. Error of the Mean is $1.11 E-11$

Statistics:

Trials

Value

Mean

10000

Median (approx.)

3.55E-10

Mode (approx.)

9.77E-11

Standard Deviation

8.41E-11

Variance

1.11E-09

Skewness

1.24E-18

Kurtosis

$7.40 \mathrm{E}+00$

Coeff. of Variability

$6.81 E+01$

Range Minimum

$3.14 \mathrm{E}+00$

Range Maximum

1.24E-12

Range Width

1.66E-08

Mean Std. Error

1.66E-08

1.11E-11

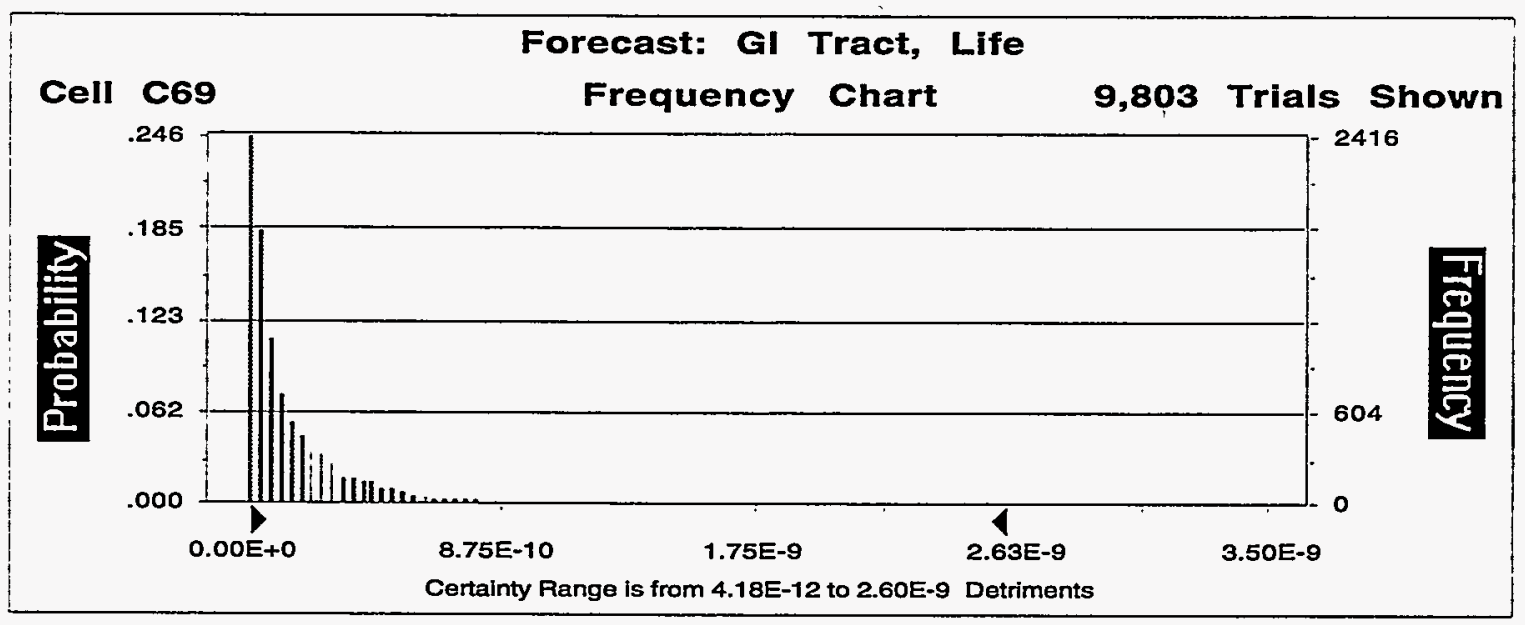


Summary:

Certainty Level is $9.50 \mathrm{E}+1 \%$

Certainty Range is from $3.53 \mathrm{E}-6$ to $2.16 \mathrm{E}-3 \mathrm{mrem} / \mathrm{y}$

Display Range is from $0.00 \mathrm{E}+0$ to $2.75 \mathrm{E}-3 \mathrm{mrem} / \mathrm{y}$

Entire Range is from 1.03E-6 to $1.38 \mathrm{E}-2 \mathrm{mrem} / \mathrm{y}$

After 10,000 Trials, the Std. Error of the Mean is 9.26E-6

Statistics:

Trials

Mean

Median (approx.)

Mode (approx.)

Standard Deviation

Variance

Skewness

Kurtosis

Coeff. of Variability

Range Minimum

Range Maximum

Range Width

Mean Std. Error
Value

10000

2.96E-04

8.14E-05

7.01E-05

9.26E-04

8.58E-07

$7.40 \mathrm{E}+00$

$6.80 \mathrm{E}+01$

$3.13 E+00$

1.03E-06

1.38E-02

1.38E-02

9.26E-06

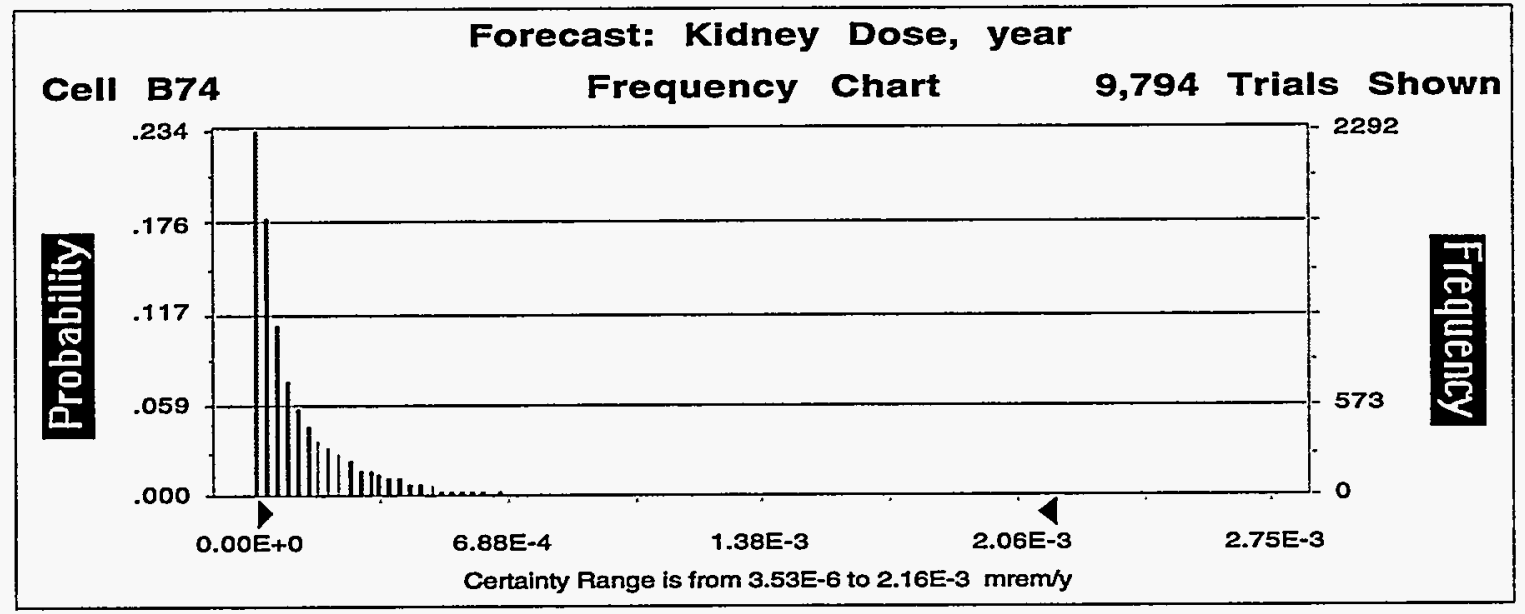


Summary:

Certainty Level is $9.50 \mathrm{E}+1 \%$

Certainty Range is from $1.74 \mathrm{E}-4$ to $1.09 \mathrm{E}-1 \mathrm{mrem}$

Display Range is from $0.00 \mathrm{E}+0$ to $1.50 \mathrm{E}-1 \mathrm{mrem}$

Entire Range is from $5.16 \mathrm{E}-5$ to $6.91 \mathrm{E}-1 \mathrm{mrem}$

After 10,000 Trials, the Std. Error of the Mean is $4.63 E-4$

Statistics:

Trials

Mean

10000

Median (approx.)

Mode (approx.)

Standard Deviation

Variance

Skewness

Kurtosis

Coeff. of Variability

1.48E-02

4.07E-03

Range Minimum

3.50E-03

4.63E-02

2.14E-03

$7.40 \mathrm{E}+00$

6.81E+01

$3.14 \mathrm{E}+00$

Range Maximum

5.16E-05

Range Width

6.91E-01

Mean Std. Error

6.91E-01

4.63E-04

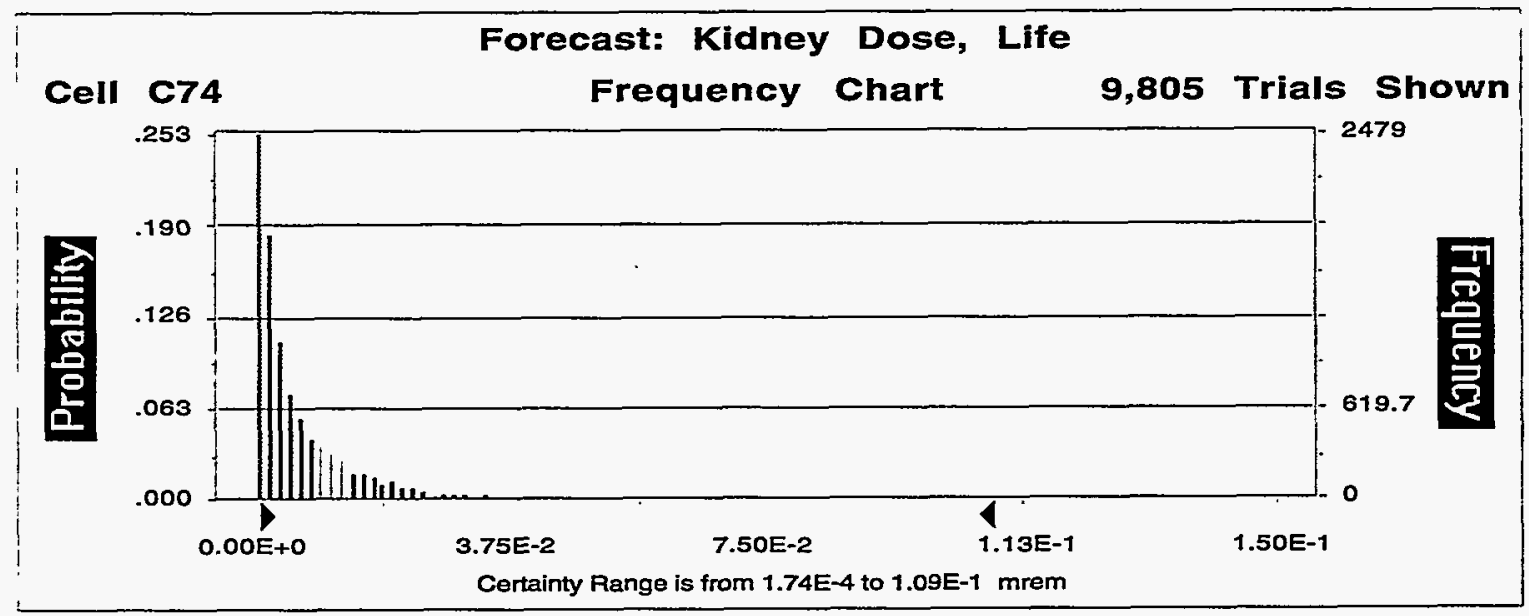


Summary:

Certainty Level is $9.50 \mathrm{E}+1 \%$

Certainty Range is from $1.72 \mathrm{E}-6$ to $1.07 \mathrm{E}-3 \mathrm{mrem} / \mathrm{y}$

Display Range is from $0.00 \mathrm{E}+0$ to $1.50 \mathrm{E}-3 \mathrm{mrem} / \mathrm{y}$

Entire Range is from $5.09 \mathrm{E}-7$ to $6.81 \mathrm{E}-3 \mathrm{mrem} / \mathrm{y}$

After 10,000 Trials, the Std. Error of the Mean is $4.57 \mathrm{E}-6$

Statistics:

Trials

Mean

Median (approx.)

Mode (approx.)

Standard Deviation

Variance

Skewness

Kurtosis

Coeff. of Variability

Range Minimum

Range Maximum

Range Width

Mean Std. Error
Value

$\overline{10000}$

$1.46 \mathrm{E}-04$

4.02E-05

3.46E-05

4.57E-04

2.09E-07

$7.40 E+00$

$6.80 E+01$

$3.13 E+00$

5.09E-07

6.81E-03

6.81E-03

4.57E-06

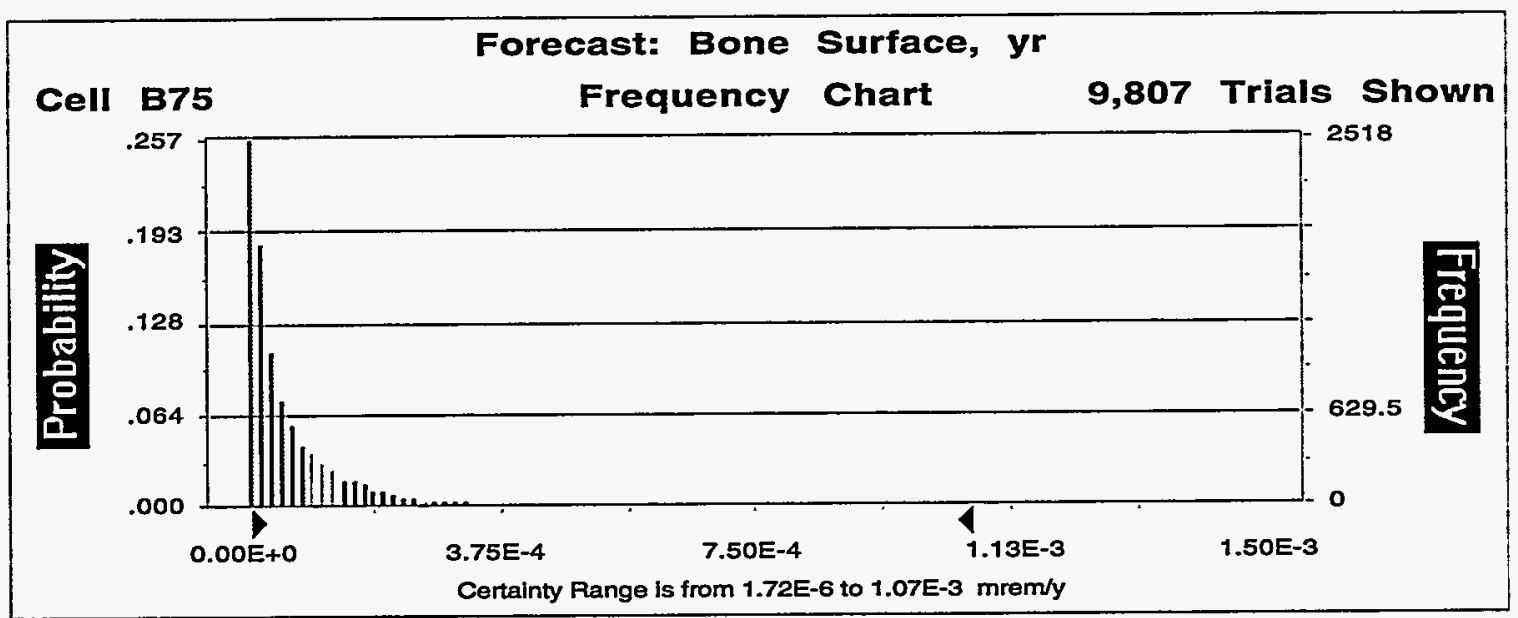


Summary:

Certainty Level is $9.50 \mathrm{E}+1 \%$

Certainty Range is from $8.64 \mathrm{E}-5$ to $5.34 \mathrm{E}-2$ mrem

Display Range is from 0.00E+0 to $7.00 \mathrm{E}-2 \mathrm{mrem}$

Entire Range is from 2.55E-5 to $3.41 \mathrm{E}-1 \mathrm{mrem}$

After 10,000 Trials, the Std. Error of the Mean is $2.28 \mathrm{E}-4$

Statistics:

Trials

Value

Mean

10000

Median (approx.)

7.29E-03

Mode (approx.)

2.01E-03

Standard Deviation

1.73E-03

Variance

2.28E-02

Skewness

5.22E-04

Kurtosis

$7.40 E+00$

Coeff. of Variability

$6.81 E+01$

Range Minimum

$3.14 \mathrm{E}+00$

Range Maximum

2.55E-05

Range Width

3.41E-01

Mean Std. Error

3.41E-01

2.28E-04

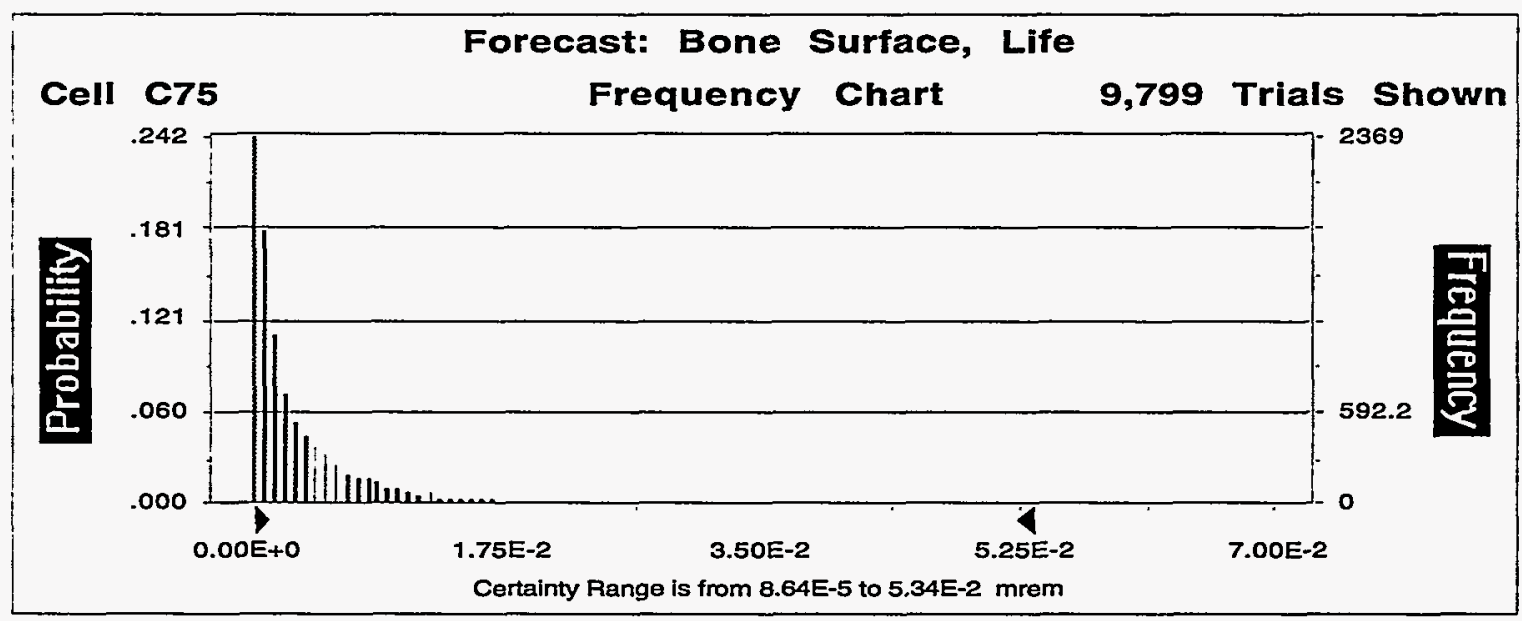


Summary:

Certainty Level is $9.50 \mathrm{E}+1 \%$

Certainty Range is from $1.16 \mathrm{E}-7$ to $7.23 \mathrm{E}-5 \mathrm{mrem} / \mathrm{yr}$

Display Range is from $0.00 \mathrm{E}+0$ to $1.00 \mathrm{E}-4 \mathrm{mrem} / \mathrm{yr}$

Entire Range is from $3.44 \mathrm{E}-8$ to $4.60 \mathrm{E}-4 \mathrm{mrem} / \mathrm{yr}$

After 10,000 Trials, the Std. Error of the Mean is 3.09E-7

Statistics:

Trials

Mean

Median (approx.)

Mode (approx.)

Standard Deviation

Variance

Skewness

Kurtosis

Coeff. of Variability

Range Minimum

Range Maximum

Range Width

Mean Std. Error
Value

10000

9.85E-06

2.71E-06

2.34E-06

3.09E-05

$9.53 \mathrm{E}-10$

$7.40 \mathrm{E}+00$

$6.80 \mathrm{E}+01$

$3.13 \mathrm{E}+00$

3.44E-08

4.60E-04

4.60E-04

3.09E-07

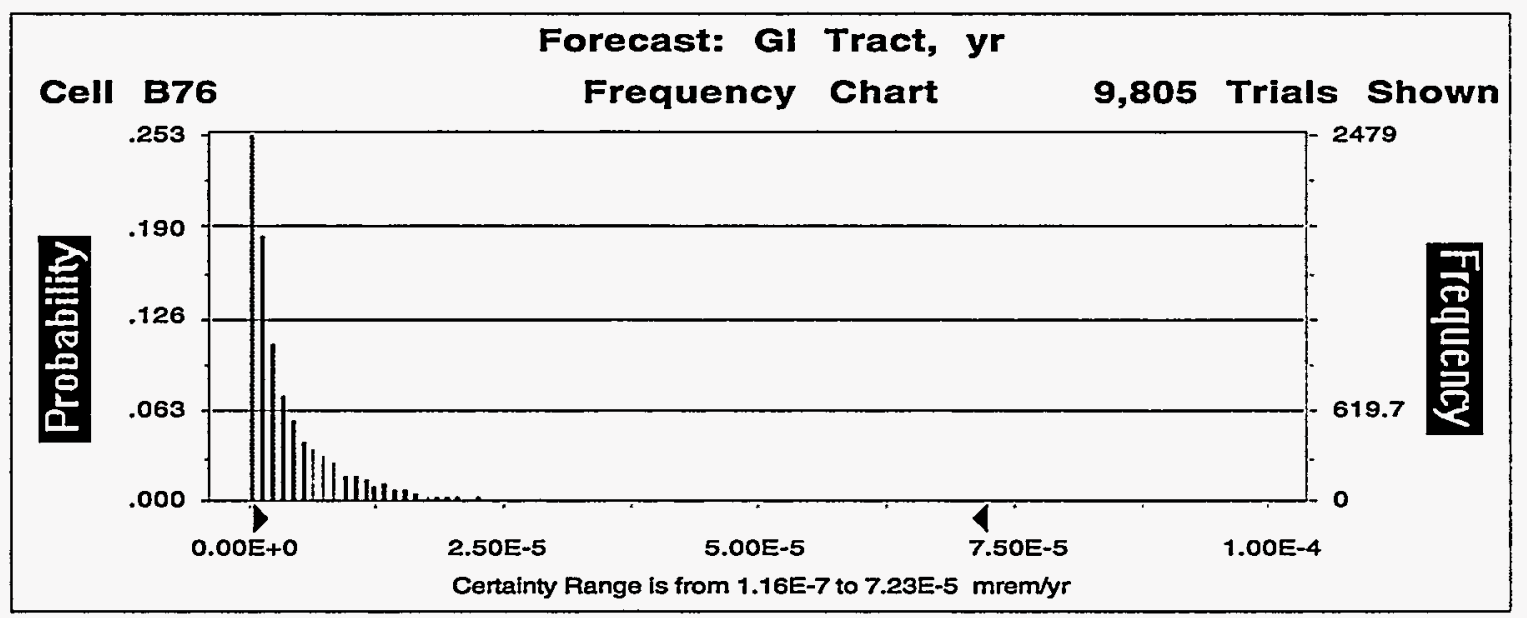


Summary:

Certainty Level is $9.50 \mathrm{E}+1 \%$

Certainty Range is from $5.79 \mathrm{E}-6$ to $3.62 \mathrm{E}-3$ mrem

Display Range is from $0.00 \mathrm{E}+0$ to $5.00 \mathrm{E}-3 \mathrm{mrem}$

Entire Range is from 1.72E-6 to $2.30 \mathrm{E}-2$ mrem

After 10,000 Trials, the Std. Error of the Mean is 1.54E-5

Statistics:

Trials

Mean

Median (approx.)

Mode (approx.)

Standard Deviation

Variance

Skewness

Kurtosis

Coeff. of Variability

Range Minimum

Range Maximum

Range Width

Mean Std. Error
Value

10000

4.92E-04

1.36E-04

1.17E-04

1.54E-03

2.38E-06

$7.40 E+00$

$6.81 E+01$

$3.14 \mathrm{E}+00$

1.72E-06

2.30E-02

2.30E-02

1.54E-05

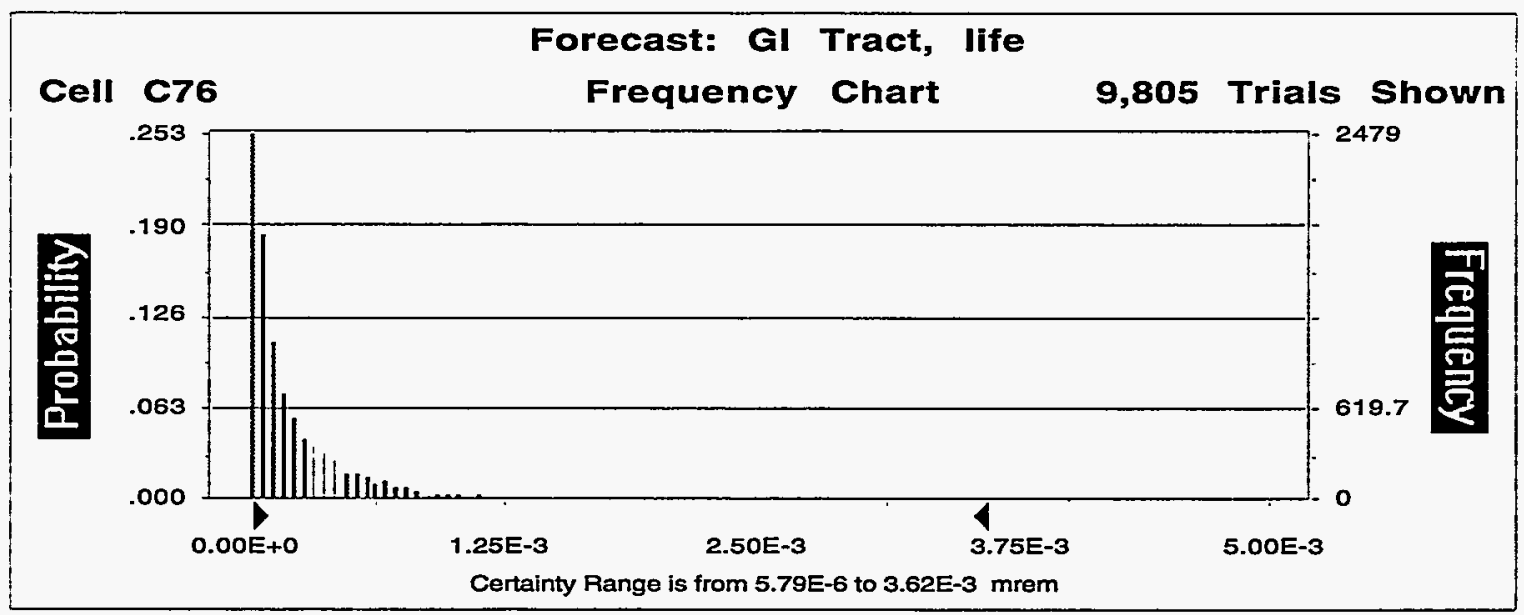




\section{Summary:}

Certainty Level is $9.50 \mathrm{E}+1 \%$

Certainty Range is from $5.22 \mathrm{E}-8$ to $3.27 \mathrm{E}-5 \mathrm{mGy} / \mathrm{d}$

Display Range is from $0.00 \mathrm{E}+0$ to $4.50 \mathrm{E}-5 \mathrm{mGy} / \mathrm{d}$

Entire Range is from $1.56 \mathrm{E}-8$ to $2.08 \mathrm{E}-4 \mathrm{mGy} / \mathrm{d}$

After 10,000 Trials, the Std. Error of the Mean is 1.40E-7

Statistics:

Trials

Mean

Median (approx.)

Mode (approx.)

Standard Deviation

Variance

Skewness

Kurtosis

Coeff. of Variability

Range Minimum

Range Maximum

Range Width

Mean Std. Error
Value

10000

4.46E-06

1.23E-06

1.06E-06

1.40E-05

$1.95 \mathrm{E}-10$

$7.40 E+00$

$6.81 E+01$

$3.14 \mathrm{E}+00$

1.56E-08

2.08E-04

2.08E-04

1.40E-07

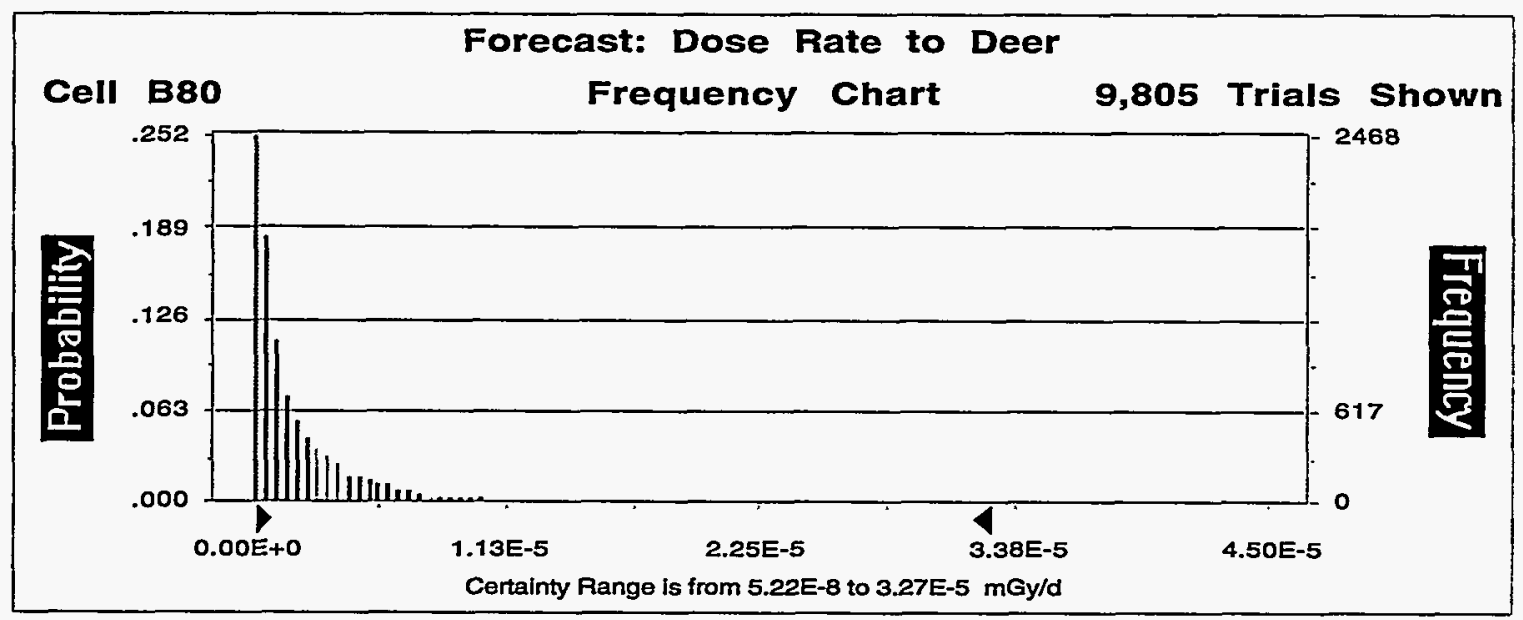


Summary:

Certainty Level is $9.50 \mathrm{E}+1 \%$

Certainty Range is from $5.22 \mathrm{E}-6$ to $3.27 \mathrm{E}-3 \mathrm{mrad} / \mathrm{d}$

Display Range is from $0.00 \mathrm{E}+0$ to $4.50 \mathrm{E}-3 \mathrm{mrad} / \mathrm{d}$

Entire Range is from $1.56 \mathrm{E}-6$ to $2.08 \mathrm{E}-2 \mathrm{mrad} / \mathrm{d}$

After 10,000 Trials, the Std. Error of the Mean is $1.40 \mathrm{E}-5$

Statistics:

Trials

Mean

Median (approx.)

Mode (approx.)

Standard Deviation

Variance

Skewness

Kurtosis

Coeff. of Variability

Range Minimum

Range Maximum

Range Width

Mean Std. Error
Value

$\overline{10000}$

4.46E-04

1.23E-04

1.06E-04

$1.40 \mathrm{E}-03$

$1.95 \mathrm{E}-06$

$7.40 \mathrm{E}+00$

$6.81 \mathrm{E}+01$

$3.14 \mathrm{E}+00$

1.56E-06

2.08E-02

2.08E-02

1.40E-05

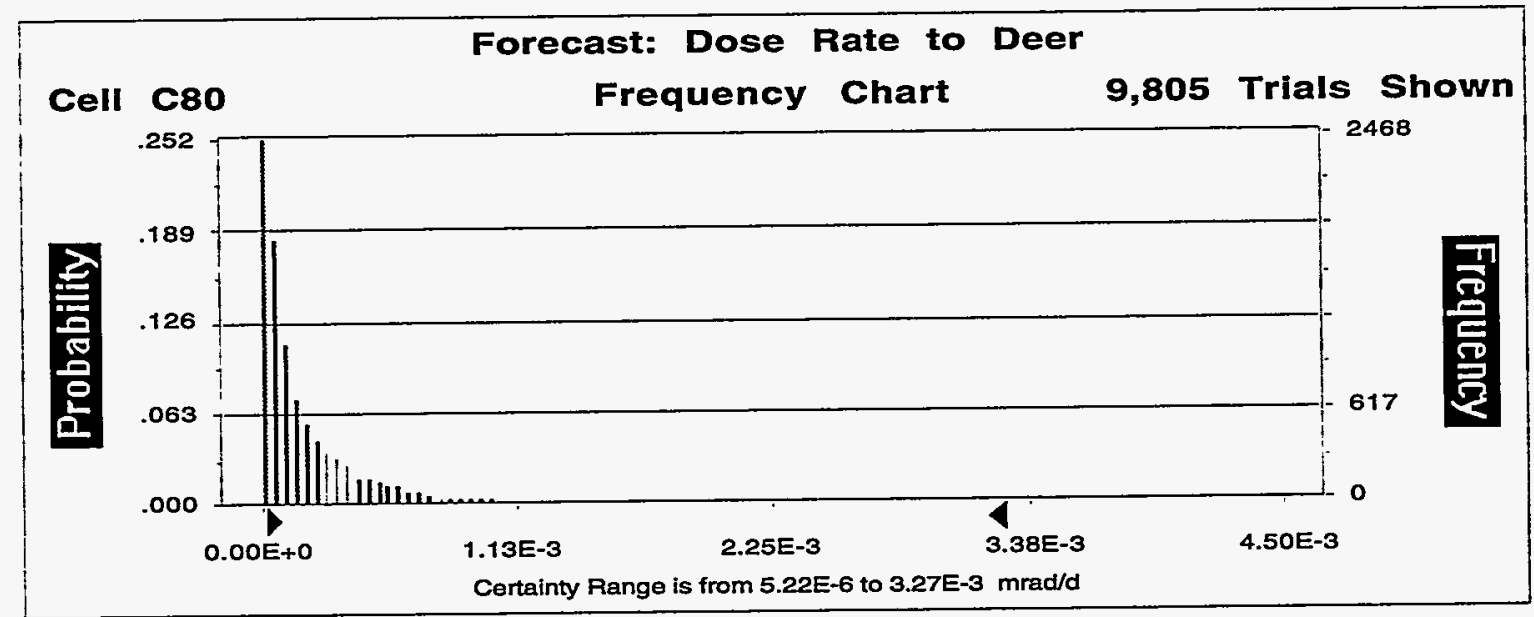


Summary:

Certainty Level is $9.50 \mathrm{E}+1 \%$

Certainty Range is from 7.84E-12 to $4.78 \mathrm{E}-9$ Detriments

Display Range is from $0.00 \mathrm{E}+0$ to $6.00 \mathrm{E}-9$ Detriments

Entire Range is from 2.28E-12 to 3.05E-8 Detriments

After 10,000 Trials, the Std. Error of the Mean is 2.05E-11

Statistics:

Trials

Mean

Median (approx.)

Mode (approx.)

Standard Deviation

Variance

Skewness

Kurtosis

Coeff. of Variability

Range Minimum

Range Maximum

Range Width

Mean Std. Error
Value

10000

6.53E-10

1.80E-10

1.55E-10

2.05E-09

4.18E-18

$7.40 \mathrm{E}+00$

$6.80 \mathrm{E}+01$

$3.13 \mathrm{E}+00$

2.28E-12

3.05E-08

3.05E-08

2.05E-11

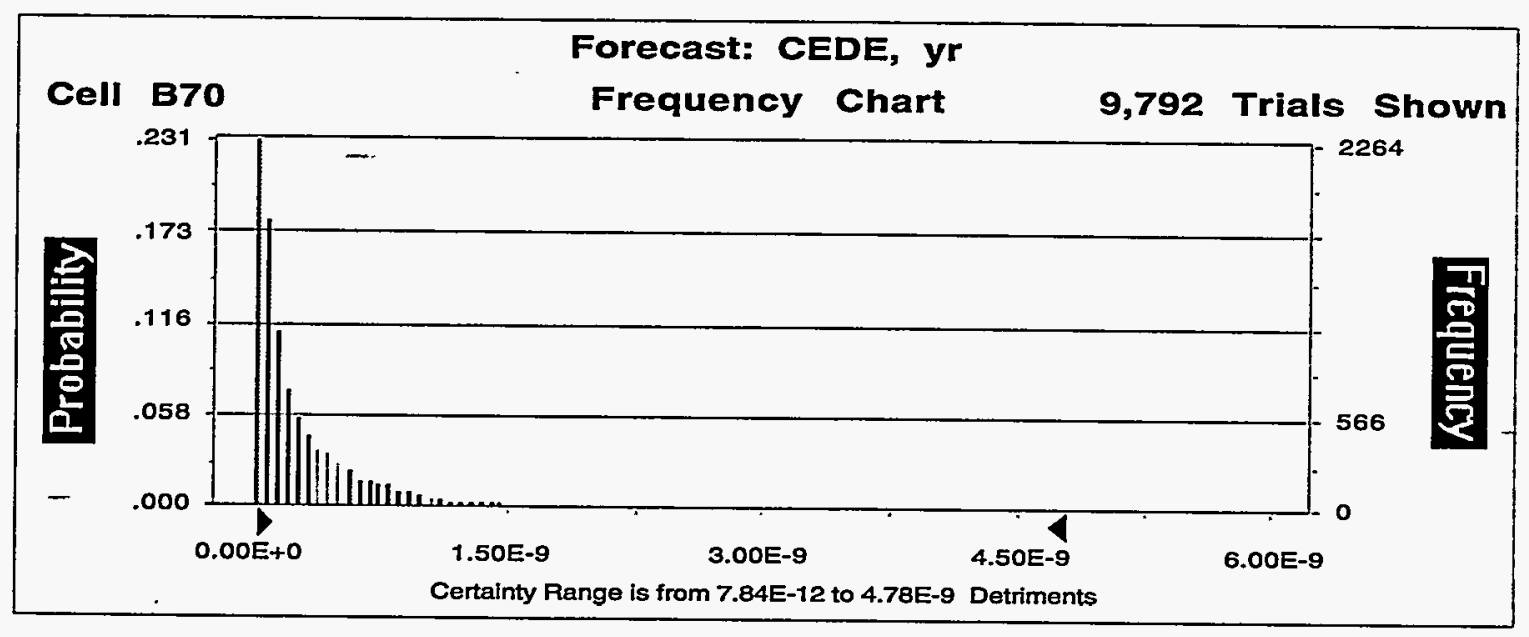


Summary:

Certainty Level is $9.50 \mathrm{E}+1 \%$

Certainty Range is from $3.92 \mathrm{E}-10$ to $2.39 \mathrm{E}-7$ Detriments

Display Range is from $0.00 E+0$ to $3.00 E-7$ Detriments

Entire Range is from 1.14E-10 to $1.53 \mathrm{E}-6$ Detriments

After 10,000 Trials, the Std. Error of the Mean is 1.02E-9

Statistics:

Trials

Mean

Median (approx.)

Mode (approx.)

Standard Deviation

Variance

Skewness

Kurtosis

Coeff. of Variability

Range Minimum

Range Maximum

Range Width

Mean Std. Error
Value

10000

3.26E-08

8.99E-09

7.74E-09

1.02E-07

1.05E-14

$7.40 E+00$

$6.81 E+01$

$3.14 \mathrm{E}+00$

1.14E-10

1.53E-06

1.52E-06

1.02E-09

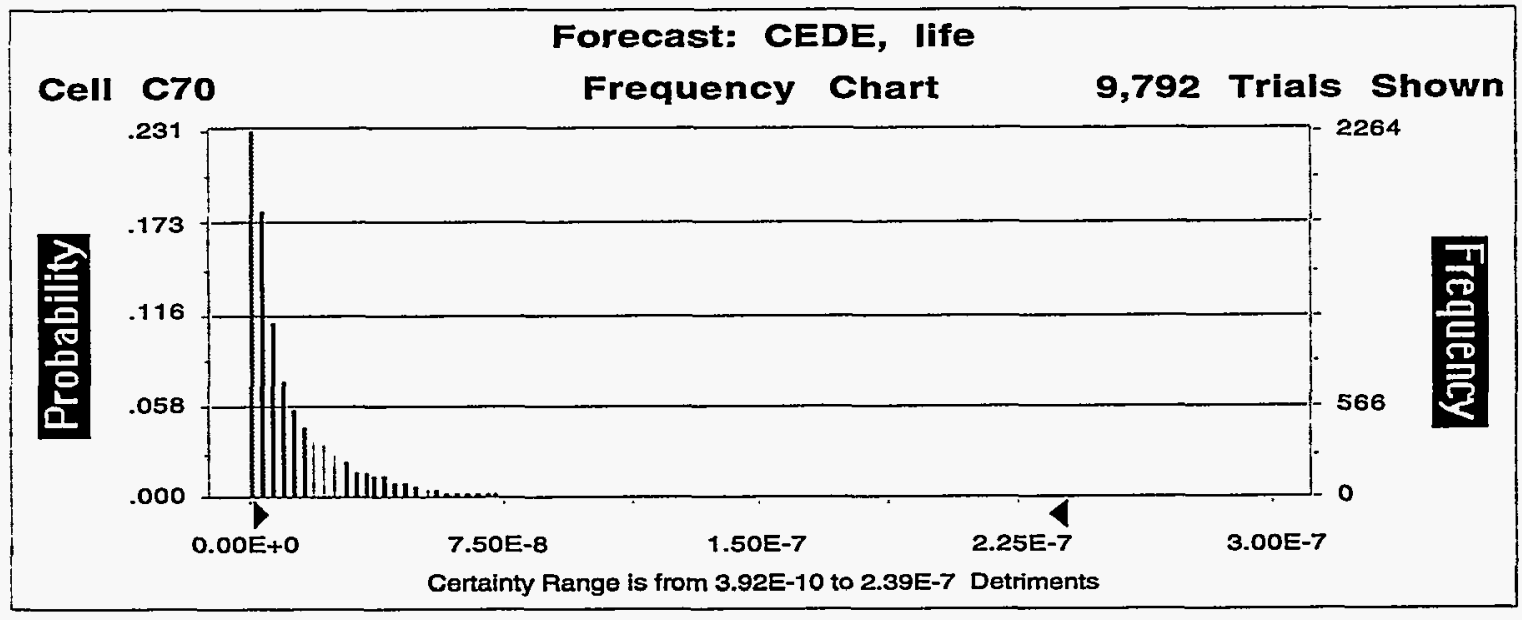


Summary:

Certainty Level is $9.50 \mathrm{E}+1 \%$

Certainty Range is from $1.07 \mathrm{E}-5$ to $6.63 \mathrm{E}-3 \mathrm{mrem} / \mathrm{yr}$

Display Range is from $0.00 \mathrm{E}+0$ to $9.00 \mathrm{E}-3 \mathrm{mrem} / \mathrm{yr}$

Entire Range is from 3.17E-6 to $4.24 \mathrm{E}-2 \mathrm{mrem} / \mathrm{yr}$

After 10,000 Trials, the Std. Error of the Mean is 2.84E-5

Statistics:

Trials

Mean.

Value

Median (approx.)

Mode (approx.)

Standard Deviation

10000

Variance

9.06E-04

Skewness

2.50E-04

Kurtosis

2.15E-04

2.84E-03

8.07E-06

$7.40 E+00$

Coeff. of Variability

$6.80 \mathrm{E}+01$

Range Minimum

3.13E+00

Range Maximum

3.17E-06

Range Width

4.24E-02

Mean Std. Error

4.24E-02

2.84E-05

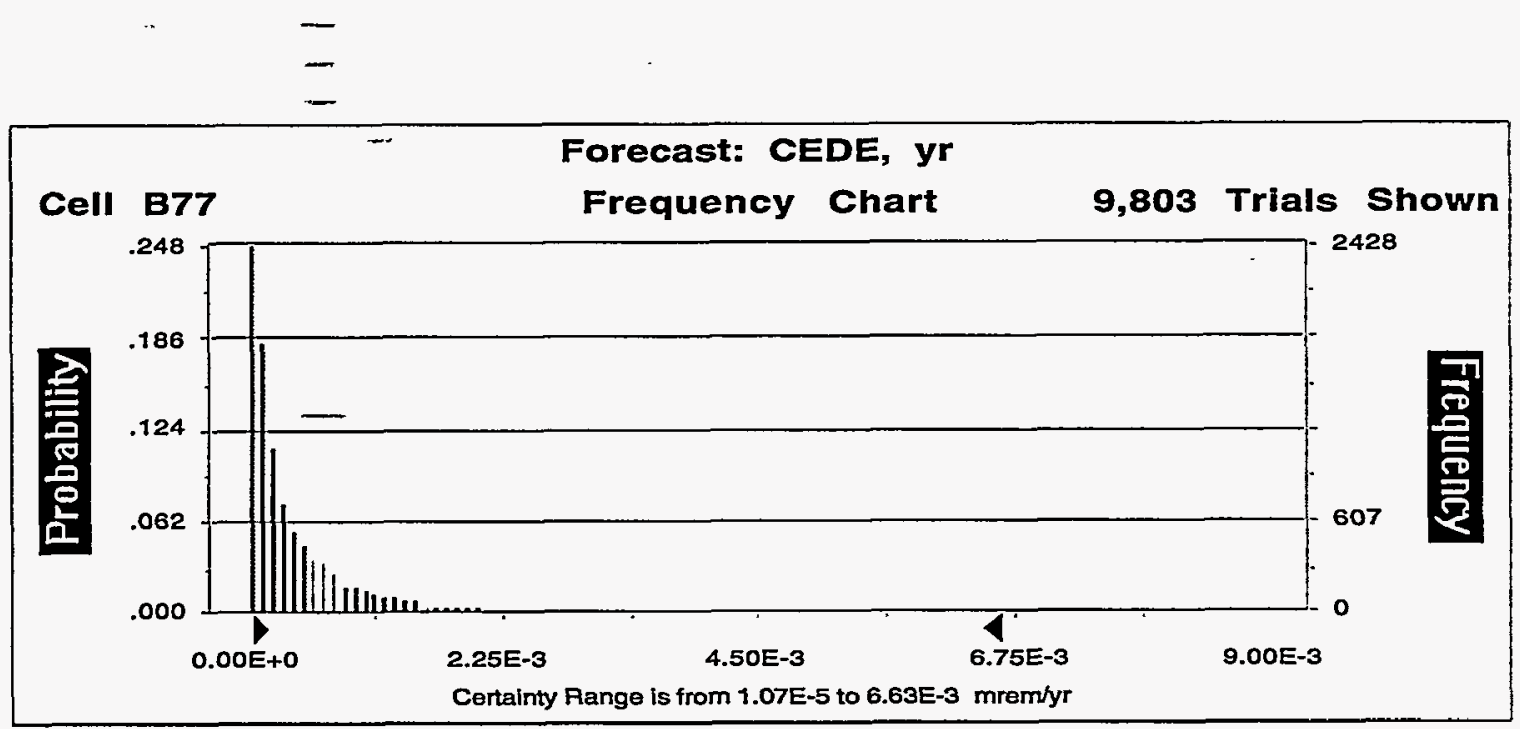


Summary:

Certainty Level is $9.50 \mathrm{E}+1 \%$

Certainty Range is from $5.35 \mathrm{E}-4$ to $3.32 \mathrm{E}-1 \mathrm{mrem}$

Display Range is from $0.00 \mathrm{E}+0$ to $4.50 \mathrm{E}-1 \mathrm{mrem}$

Entire Range is from $1.58 \mathrm{E}-4$ to $2.12 \mathrm{E}+0$ mrem

After 10,000 Trials, the Std. Error of the Mean is $1.42 \mathrm{E}-3$

Statistics:

Trials

Value

10000

Mean

4.53E-02

Median (approx.)

$1.25 \mathrm{E}-02$

Mode (approx.)

1.07E-02

Standard Deviation

1.42E-01

Variance

2.02E-02

Skewness

$7.40 \mathrm{E}+00$

Kurtosis

$6.80 E+01$

Coeff. of Variability

$3.13 E+00$

Range Minimum

$1.58 \mathrm{E}-04$

Range Maximum

$2.12 \mathrm{E}+00$

Range Width

$2.12 \mathrm{E}+00$

Mean Std. Error

1.42E-03

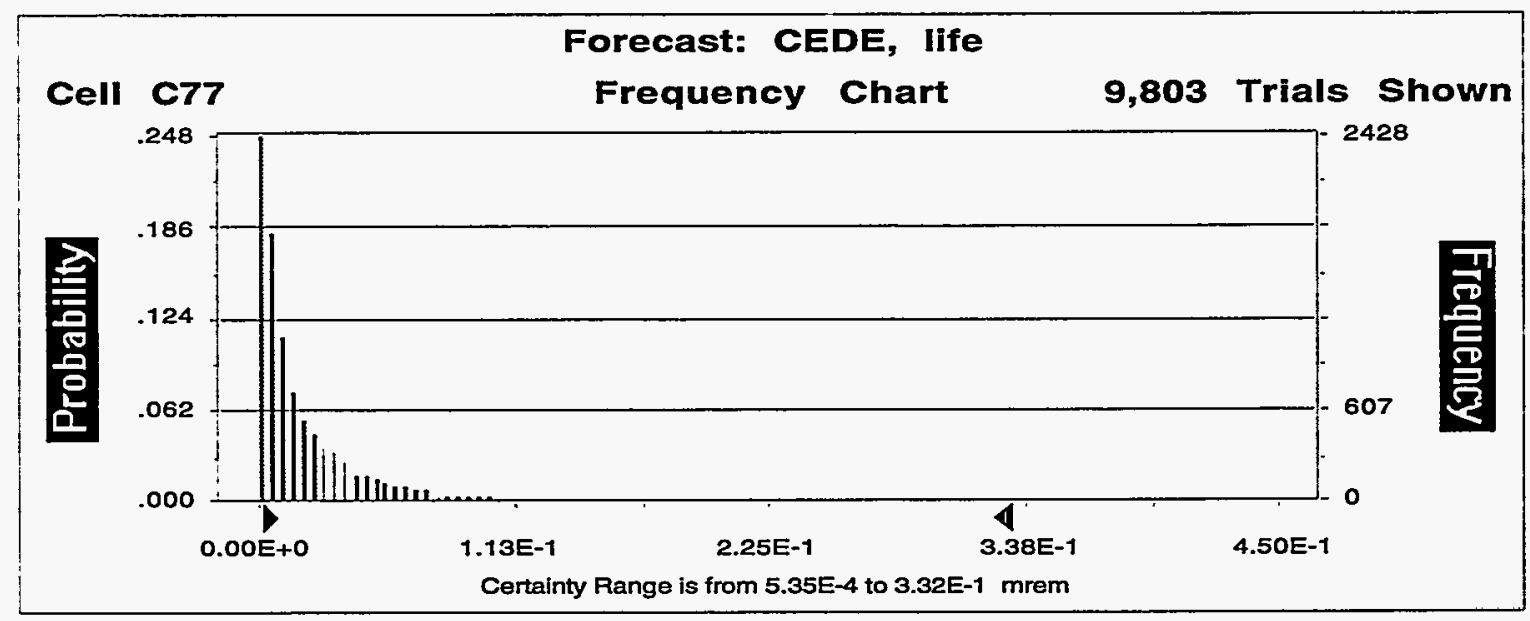




\section{Summary:}

Certainty Level is $9.50 \mathrm{E}+1 \%$

Certainty Range is from $2.45 \mathrm{E}-2$ to $1.52 \mathrm{E}+1 \mathrm{pCi} / \mathrm{kg}$

Display Range is from $0.00 \mathrm{E}+0$ to $2.00 \mathrm{E}+1 \mathrm{pCi} / \mathrm{kg}$

Entire Range is from $7.24 \mathrm{E}-3$ to $9.69 \mathrm{E}+1 \mathrm{pCi} / \mathrm{kg}$

After 10,000 Trials, the Std. Error of the Mean is $6.50 \mathrm{E}-2$

\section{Statistics: \\ Trials \\ Mean \\ Median (approx.) \\ Mode (approx.) \\ Standard Deviation \\ Variance \\ Skewness \\ Kurtosis \\ Coeff. of Variability \\ Range Minimum \\ Range Maximum \\ Range Width \\ Mean Std. Error}

Value

10000

2.07E+00

5.72E-01

4.92E-01

$6.50 \mathrm{E}+00$

4.23E+01

$7.40 \mathrm{E}+00$

$6.80 E+01$

$3.13 E+00$

7.24E-03

$9.69 E+01$

$9.69 \mathrm{E}+01$

$6.50 \mathrm{E}-02$

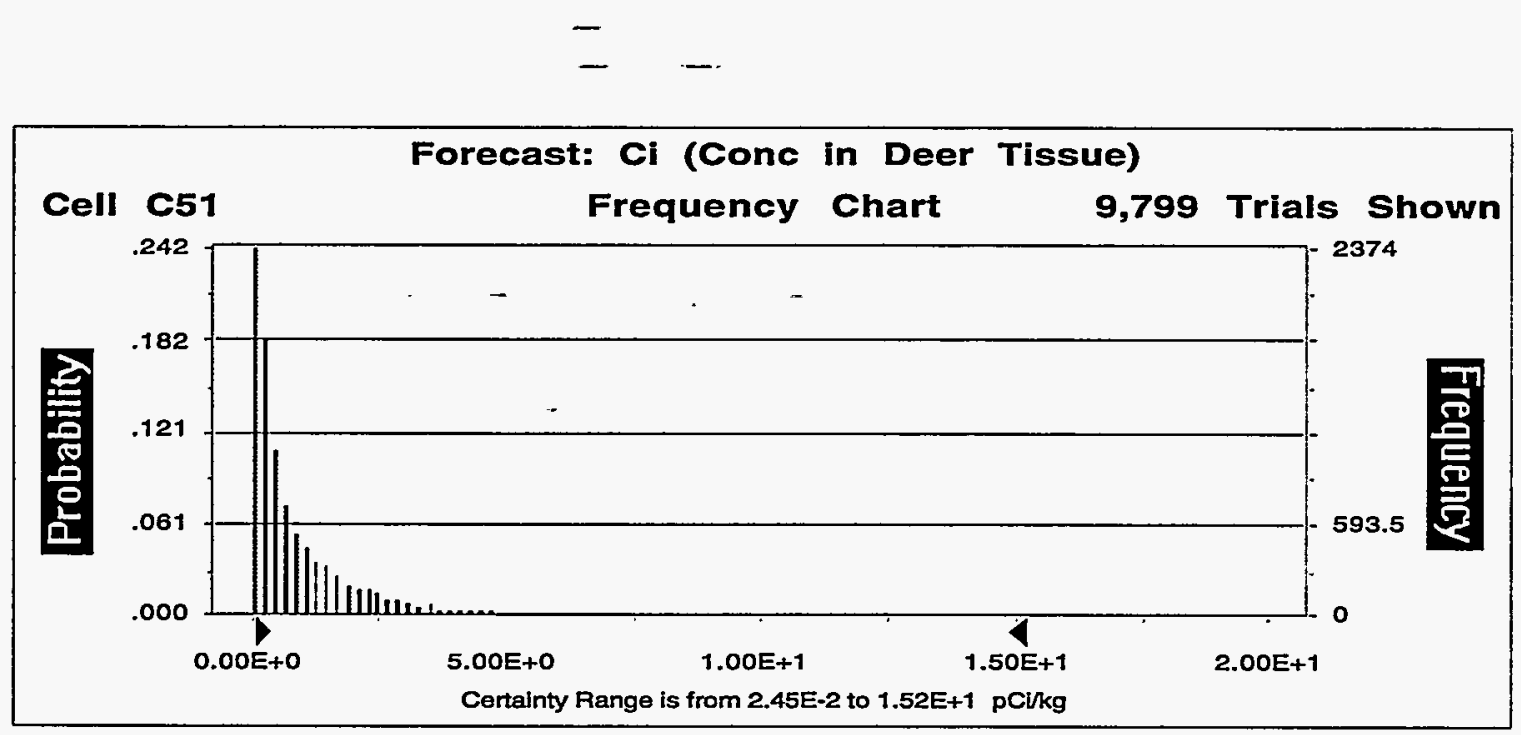




\section{Assumptions}

Assumption: Soil Conc (pCi/g)

Cell: B34

Custom distribution with parameters:

Single point 2.00

Single point

4.00

6.00

Single point

8.00

Single point

10.00

12.00

Single point

14.00

Single point

16.00

18.00

Single point

20.00

40.00

Single point

60.00

80.00

Single point

100.00

Single point

300.00

Relative Prob.

0.232

0.082

0.049

0.085

0.105

0.095

0.042

0.078

0.059

0.043

0.078

0.0033

0.0065

0.007

0.0400

1.0048

Mean value in simulation was 23.52

Source: APG ERM data and Field Collection Data 
Assumption: Water Concentration, $\mathrm{C}_{\mathrm{iw}}(\mathrm{pCi} / \mathrm{L})$

Uniform distribution with parameters:
Minimum
5.00E-01
Maximum
$3.00 \mathrm{E}+00$

Mean value in simulation was $1.76 \mathrm{E}+0$

Source: Cothren et al, 1983; Cothren and Lappenbusch, 1983.

Assumption: Water Intake, $Q_{w}(p C i / L)$

Cell: B18

Triangular distribution with parameters:

\begin{tabular}{|c|}
\hline Minimum \\
\hline $\begin{array}{l}\text { Likeliest } \\
\text { Maximum }\end{array}$ \\
\hline
\end{tabular}

Selected range is from 1.40 to 13.50

Mean value in simulation was 6.32

Source: Lautier et al, 1988. 
Normal distribution with parameters:

Mean

24.00

Standard Dev.

3.00

Selected range is from -Infinity to +Infinity

Mean value in simulation was 23.97

Source: Alldredge et al, 1974.

Assumption: Dust mass (pCi/m $\left.{ }^{2}-\mathrm{d}\right)$

Cell: B27

Uniform distribution with parameters:
Minimum
3.00E-04

Maximum

$9.20 \mathrm{E}-02$

Mean value in simulation was $4.63 \mathrm{E}-2$

Source: Dust cloud measurements, YPG, December, 1993.

Assumption: Soil Ingestion Rate (kg/d)

Cell: B36

Uniform distribution with parameters:
Minimum
0.01
Maximum
0.05

Mean value in simulation was 0.03

Source: Alldredge and Arthur, 1979; Arthur and GAtes, 1988.

Assumption: Weathering time, $t_{w}$ (d)

Cell: B45

Uniform distribution with parameters:

$\begin{array}{ll}\text { Minimum } & 1.00 \\ \text { Maximum } & 20.00\end{array}$

Mean value in simulation was 10.48

Source: NCRP, 1984.

End of Assumptions 


\section{Appendix C}

Aquatic Data from APG 
Table C-1. Data for aquatic samples collected at APG. Shaded values in Mass Ratio column indicate data that fell within laboratory QA/QC. Unshaded values fell outside Laboratory QA/QC and are invalid.

\begin{tabular}{|c|c|c|c|c|c|c|c|c|}
\hline Species & ID & Series & $\begin{array}{c}\text { U-238 } \\
(\mu \mathrm{g} / \mathrm{kg})\end{array}$ & $\begin{array}{c}U-235 \\
(\mu \mathrm{g} / \mathrm{kg})\end{array}$ & $\begin{array}{l}\text { U-235/U-238 } \\
\text { Mass Ratio }\end{array}$ & $\begin{array}{l}\text { U-238 Conc } \\
\text { (pCi/g) }\end{array}$ & \# Individuals & \# Measurements \\
\hline \multicolumn{9}{|l|}{ Anchovy } \\
\hline & 3069 & & 55.00 & & & 1.87E-02 & 2 & \\
\hline & 34 & & 260.00 & & & 8.84E-02 & 21 & \\
\hline & 53 & & 810.00 & 10.00 & 0.0123 & 2.75E-01 & 48 & \\
\hline & 80 & & 100.00 & & & $3.40 E-02$ & 20 & \\
\hline & 81 & & 59.00 & & & 2.01E-02 & 6 & \\
\hline & 112 & & 15.00 & & & $5.10 \mathrm{E}-03$ & 11 & \\
\hline & 113 & & 44.00 & & & $1.50 \mathrm{E}-02$ & 18 & \\
\hline Ave: & & & 191.86 & & & $6.52 \mathrm{E}-02$ & 126 & \\
\hline$s d$ & & & & & & $9.66 \mathrm{E}-02$ & & 7 \\
\hline & & & & & & & & \\
\hline \multicolumn{9}{|l|}{ Blue Crab } \\
\hline & 3088 & $1-3$, hepato & 69.00 & & & 2.35E-02 & 3 & \\
\hline & 3094 & $1-3$, muscle & 66.00 & & & $2.24 \mathrm{E}-02$ & 3 & \\
\hline & 3130 & $1-2$, muscle & 32.00 & & & $1.09 \mathrm{E}-02$ & 2 & \\
\hline & 83 & & 86.00 & & & $2.92 E-02$ & 1 & \\
\hline & 173 & & 200.00 & -1.75 & & $6.80 \mathrm{E}-02$ & 1 & \\
\hline & 174 & $\bar{A}$ & 89.00 & & & $3.03 E-02$ & 1 & \\
\hline & 174 & B & 130.00 & & & $4.42 E-02$ & 1 & \\
\hline Ave: & & & 96.00 & & & $3.26 \mathrm{E}-02$ & 12 & \\
\hline$s d$ & & & & & & $1.85 \mathrm{E}-02$ & & \\
\hline Muscle & & & $9.46 \mathrm{E}+01$ & & & $3.22 E-02$ & 8 & 7 \\
\hline \multirow{2}{*}{\multicolumn{9}{|c|}{ Bullhead }} \\
\hline & & & & & & & & \\
\hline & 3103 & & 380.00 & & & $1.29 E-01$ & 1 & \\
\hline & 143 & & 180.00 & & & $6.12 E-02$ & 2 & \\
\hline & 144 & & 280.00 & & & 9.52E-02 & 2 & \\
\hline & 145 & & 250.00 & & & $8.50 E-02$ & 1 & \\
\hline Ave: & & & $2.73 E+02$ & & & 9.27E-02 & 6 & \\
\hline$s d$ & & & & & & $2.82 E-02$ & & 4 \\
\hline & & & & & & & & \\
\hline \multicolumn{9}{|l|}{ Belastoma } \\
\hline & 138 & & 280.00 & & & $9.52 E-02$ & 2 & 1 \\
\hline & & & & & & & & \\
\hline \multicolumn{9}{|l|}{ Carp } \\
\hline & 3001 & \#2, BONES & 400.00 & & & 1.36E-01 & 1 & \\
\hline & 3003 & \#1 LIVER & 200.00 & & & $6.80 \mathrm{E}-02$ & 1 & \\
\hline & 3004 & \#2 GUT & 24.00 & & & 8.16E-03 & 1 & \\
\hline & 3005 & \#1 GUT & 62.00 & & & 2.11E-02 & 1 & \\
\hline & 3008 & \#2LIVER & 490.00 & & & 1.67E-01 & 1 & \\
\hline & 3018 & \#2FILLETS & 14.00 & & & $4.76 E-03$ & 1 & \\
\hline Ave: & & & $1.98 \mathrm{E}+02$ & & & $6.74 E-02$ & 6 & \\
\hline$s d$ & & & & & & $6.95 E-02$ & & \\
\hline LIVER & & & $3.45 E+02$ & & & 1.17E-01 & 2 & \\
\hline GUT & & & $4.30 \mathrm{E}+01$ & & & 1.46E-02 & 2 & 6 \\
\hline & & & & & & & & \\
\hline
\end{tabular}


Table C-1. (cont.)

\begin{tabular}{|c|c|c|c|c|c|c|c|c|}
\hline Species & ID & Series & $\begin{array}{c}\text { U-238 } \\
(\mu \mathrm{g} / \mathrm{kg})\end{array}$ & $\begin{array}{c}\text { U-235 } \\
(\mu \mathrm{g} / \mathrm{kg})\end{array}$ & $\begin{array}{l}\text { U-235/U-238 } \\
\text { Mass Ratio }\end{array}$ & $\begin{array}{l}\text { U-238 Conc } \\
\text { (pCi/g) }\end{array}$ & \# Individuals & \# Measurements \\
\hline \multicolumn{9}{|l|}{ Catfish } \\
\hline & 3080 & liver & -1.00 & & & $-3.40 \mathrm{E}-04$ & 1 & \\
\hline & 3082 & fillets & 230.00 & & & $7.82 E-02$ & 1 & 2 \\
\hline \multicolumn{9}{|l|}{ Ave: } \\
\hline & & & & & & & & \\
\hline \multicolumn{9}{|l|}{ Cattail } \\
\hline & 224 & & 10000.00 & 46.00 & 0.0046 & $3.40 \mathrm{E}+00$ & 1 & \\
\hline & 225 & & 1900.00 & 13.00 & 0.0068 & $6.46 \mathrm{E}-01$ & 1 & \\
\hline Ave: & & & $5.95 E+03$ & & & $2.02 E+00$ & $\overline{2}$ & \\
\hline$s d$ & & & & & & $1.95 E+00$ & & 2 \\
\hline & & & & & & & & \\
\hline \multicolumn{9}{|l|}{ Chub } \\
\hline & 160 & & 50.00 & & & 1.70E-02 & 1 & \\
\hline & 161 & & 34.00 & & & 1.16E-02 & 1 & \\
\hline Ave: & & & $4.20 \mathrm{E}+01$ & & & 1.43E-02 & 2 & \\
\hline$s d$ & & & & & & $3.85 E-03$ & & 2 \\
\hline & & & & & & & & \\
\hline \multicolumn{9}{|l|}{ Clam } \\
\hline & 3035 & & 120.00 & & & $4.08 E-02$ & 1 & \\
\hline & 3059 & & 250.00 & & & $8.50 \mathrm{E}-02$ & 2 & \\
\hline & 3061 & & 360.00 & & & $1.22 \mathrm{E}-01$ & 2 & \\
\hline & 3062 & & 300.00 & & & $1.02 \mathrm{E}-01$ & 1 & \\
\hline & 82 & B & 10.00 & & & $3.40 \mathrm{E}-03$ & 2 & \\
\hline & 82 & $\bar{A}$ & 30.00 & & & $1.02 \mathrm{E}-02$ & 2 & \\
\hline Avo: & & & $1.78 \mathrm{E}+02$ & & & $6.06 \mathrm{E}-02$ & 10 & \\
\hline sd & & & & & & 4.97E-02 & & \\
\hline Ave, -dups & & & & & & $4.46 \mathrm{E}-02$ & 8 & 6 \\
\hline & & & & & & & & \\
\hline \multicolumn{9}{|l|}{ Crawfish } \\
\hline & 164 & & 310.00 & & & 1.05E-01 & 1 & 1 \\
\hline & & & & & & & & \\
\hline \multicolumn{9}{|l|}{ Darter } \\
\hline & 104 & & 31.00 & & & 1.05E-02 & 1 & 1 \\
\hline \multicolumn{9}{|l|}{ Eel } \\
\hline & 175 & & 120.00 & & & $4.08 E-02$ & & 1 \\
\hline & & & & & & & & \\
\hline \multirow[t]{3}{*}{ Frog } & & & & & $\cdots$ & & & \\
\hline & 3087 & & 150.00 & & & $5.10 \mathrm{E}-02$ & 1 & \\
\hline & 3124 & & 5.00 & & & $1.70 \mathrm{E}-03$ & 1 & 2 \\
\hline Ave: & & & $7.75 E+01$ & & & 2.64E-02 & 2 & \\
\hline \multirow[t]{3}{*}{ sd } & & & & & & 3.49E-02 & & \\
\hline & & & & & & & & \\
\hline & & & & & & & & \\
\hline \multicolumn{9}{|l|}{ Tadpoles } \\
\hline & 170 & & 510.00 & & & $1.73 E-01$ & 1 & \\
\hline & 3093 & & 710.00 & 56.00 & 0.0789 & $2.41 E-01$ & 2 & \\
\hline & 3102 & & 690.00 & & & 2.35E-01 & 2 & \\
\hline & 3111 & & 240.00 & & & $8.16 E-02$ & 1 & \\
\hline & 3154 & & 440.00 & & & $1.50 E-01$ & 12 & \\
\hline Avo: & & & $5.20 E+02$ & & & 1.76E-01 & 18 & \\
\hline
\end{tabular}


Table C-1. (cont.)

\begin{tabular}{|c|c|c|c|c|c|c|c|c|}
\hline Species & ID & Series & $\begin{array}{c}\mathrm{U}-238 \\
(\mu \mathrm{g} / \mathrm{kg})\end{array}$ & $\begin{array}{c}\text { U-235 } \\
(\mu \mathrm{g} / \mathrm{kg})\end{array}$ & $\begin{array}{l}\text { U-235J-238 } \\
\text { Mass Ratio }\end{array}$ & $\begin{array}{l}\text { U-238 Conc } \\
\text { (pCi/g) }\end{array}$ & \# Individuals & \# Measurements \\
\hline sd & & & & & & $6.58 \mathrm{E}-02$ & & 5 \\
\hline \multirow{2}{*}{\multicolumn{9}{|c|}{ Killifish }} \\
\hline & & & & & & & & \\
\hline & 3100 & & 5.00 & & & $1.70 \mathrm{E}-03$ & 14 & \\
\hline & 169 & & 1000.00 & 12.00 & 0.0120 & $3.40 E-01$ & 7 & \\
\hline & 172 & & 86.00 & & & $2.92 E-02$ & 8 & \\
\hline Ave: & & & $3.64 E+02$ & & & $1.24 \mathrm{E}-01$ & 29 & \\
\hline$s d$ & & & & & & $1.88 \mathrm{E}-01$ & & 3 \\
\hline & & & & & & & & \\
\hline \multicolumn{9}{|l|}{ Millfoil } \\
\hline & 3028 & & 2100.00 & 6.00 & 0.0029 & $7.14 \mathrm{E}-01$ & & \\
\hline & 3042 & & 800.00 & 40.00 & 0.0500 & $2.72 \mathrm{E}-01$ & & \\
\hline Ave: & & & $1.45 E+03$ & & & $4.93 \mathrm{E}-01$ & & \\
\hline$s d$ & & & & & & 3.13E-01 & & 2 \\
\hline & & & & & & & & \\
\hline \multicolumn{9}{|l|}{ Mosquito fish } \\
\hline & 162 & & 25.00 & & & $8.50 \mathrm{E}-03$ & $\overline{3}$ & 1 \\
\hline & & & & & & & & \\
\hline \multicolumn{9}{|l|}{ Hogchocker } \\
\hline & 3044 & & 160.00 & & & $5.44 \mathrm{E}-02$ & 4 & \\
\hline & 3045 & & 160.00 & & & $5.44 \mathrm{E}-02$ & 1 & \\
\hline & 27 & & 26.00 & & & $8.84 E-03$ & 1 & \\
\hline & 38 & & 160.00 & & & $5.44 \mathrm{E}-02$ & 1 & \\
\hline & 41 & & 180.00 & & & $6.12 E-02$ & 1 & \\
\hline & $\overline{72}$ & & 61.00 & & & $2.07 \mathrm{E}-02$ & 1 & \\
\hline & 84 & & 110.00 & & & $3.74 \mathrm{E}-02$ & 1 & \\
\hline Ave: & & & $1.22 E+02$ & & & $4.16 \mathrm{E}-02$ & 9 & 7 \\
\hline$s d$ & & & & & & $2.00 \mathrm{E}-02$ & & \\
\hline & & & & & & & & \\
\hline \multicolumn{9}{|l|}{ Heron } \\
\hline & 3104 & LIVER & 12.00 & & & $4.08 \mathrm{E}-03$ & & \\
\hline & 3106 & GUT & 30.00 & 2.70 & 0.0900 & $1.10 \mathrm{E}-02$ & & \\
\hline & 3107 & VERTEBRAE & 5.00 & & & $1.70 \mathrm{E}-03$ & & \\
\hline & 3108 & KIDNEY & 250 & & & $8.50 \mathrm{E}-04$ & & 4 \\
\hline & & & & & & & & \\
\hline \multicolumn{9}{|l|}{ Water Beetles } \\
\hline \multirow{2}{*}{\multicolumn{9}{|c|}{ Shiner }} \\
\hline & & & & & & & & \\
\hline & & & & & & & & \\
\hline \multicolumn{9}{|l|}{ Pickerel } \\
\hline & 3118 & & 7.50 & & & $2.55 \mathrm{E}-03$ & 1 & \\
\hline & 3120 & & 15.00 & & & $5.10 \mathrm{E}-03$ & 1 & \\
\hline & & & $1.13 E+01$ & & & $3.83 \mathrm{E}-03$ & 2 & 2 \\
\hline & & & & & & $1.80 \mathrm{E}-03$ & & \\
\hline & & & & & & & & \\
\hline \multicolumn{9}{|l|}{ Pickrell Weed } \\
\hline & 3065 & & 6300.00 & 13.00 & 0.0021 & $2.14 E+00$ & & 1 \\
\hline & & & & & & & & \\
\hline
\end{tabular}


Table C-1. (cont.)

\begin{tabular}{|c|c|c|c|c|c|c|c|c|}
\hline Species & ID & Series & $\begin{array}{c}\text { U-238 } \\
(\mu \dot{g} / \mathrm{kg})\end{array}$ & $\begin{array}{c}\text { U-235 } \\
(\mu \mathrm{g} / \mathrm{kg})\end{array}$ & $\begin{array}{l}\text { U-235几-238 } \\
\text { Mass Ratio }\end{array}$ & $\begin{array}{l}\text { U-238 Conc } \\
\text { (pCi/g) }\end{array}$ & \# Individuals & \# Measurements \\
\hline \multicolumn{9}{|l|}{ Seston } \\
\hline & 3036 & & 3800.00 & 18.00 & 0.0047 & $1.29 E+00$ & & \\
\hline & 3038 & & 2900.00 & 18.00 & 0.0062 & $9.86 E-01$ & & \\
\hline & 3067 & & 6300.00 & 39.10 & 0.0062 & $2.14 E+00$ & & \\
\hline & 214 & & 1600.00 & 12.00 & 0.0075 & $5.44 E-01$ & & \\
\hline & 218 & & 3100.00 & 22.00 & 0.0071 & 1.05E+00 & & \\
\hline & 220 & & 5000.00 & 42.00 & 0.0084 & $1.70 E+00$ & & \\
\hline & 222 & & 2100.00 & 14.00 & 0.0067 & 7.14E-01 & & \\
\hline Ave: & & & $3.54 \mathrm{E}+03$ & & & $1.20 \mathrm{E}+00$ & 7 & 7 \\
\hline sd & & & & & & $5.60 \mathrm{E}-01$ & & \\
\hline & & & & & & & & \\
\hline \multicolumn{9}{|l|}{ Sunfish } \\
\hline & 39 & & 39.00 & & & 1.33E-02 & 1 & \\
\hline & 40 & & 94.00 & & & $3.20 E-02$ & 1 & \\
\hline & 105 & $\bar{A}$ & 43.00 & & & $1.46 E-02$ & 1 & \\
\hline & 105 & $\bar{B}$ & 78.00 & & & $2.65 \mathrm{E}-02$ & 1 & \\
\hline & 106 & & 160.00 & & & 5.44E-02 & 1 & \\
\hline & 107 & & 58.00 & & & 1.97E-02 & 1 & \\
\hline & 108 & $\bar{A}$ & 130.00 & & & $4.42 E-02$ & 1 & \\
\hline & 108 & B & 160.00 & & & 5.44E-02 & 1 & \\
\hline & 109 & & 87.00 & & & 2.96E-02 & 1 & \\
\hline & 110 & & 59.00 & & & 2.01E-02 & 1 & - \\
\hline & 111 & & 130.00 & & & 4.42E-02 & 1 & \\
\hline & 114 & & 140.00 & & & $4.76 \mathrm{E}-02$ & 1 & \\
\hline & 115 & & 36.00 & & & $1.22 E-02$ & 1 & \\
\hline & 116 & & 49.00 & & & 1.67E-02 & 1 & \\
\hline & 117 & $:$ & 93.00 & & & $3.16 E-02$ & 1 & \\
\hline & 118 & $\vdots$ & 38.00 & & & $1.29 \mathrm{E}-02$ & 1 & \\
\hline & 119 & & 59.00 & & & $2.01 \mathrm{E}-02$ & 1 & \\
\hline & 120 & $\mathrm{~B}$ & 69.00 & & & $2.35 E-02$ & 1 & \\
\hline & 120 & $A$ & 170.00 & & & $5.78 E-02$ & 1 & \\
\hline & 121 & 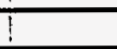 & 130.00 & & & $4.42 E-02$ & 1 & \\
\hline & 122 & 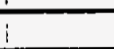 & 170.00 & & & $5.78 E-02$ & 1 & \\
\hline & 123 & & 170.00 & & & $5.78 E-02$ & 1 & \\
\hline & 124 & & 31.00 & & & $1.05 \mathrm{E}-02$ & 1 & \\
\hline & 125 & & 200.00 & & & $6.80 E-02$ & 1 & \\
\hline & 126 & & 810.00 & 5.50 & $0.0068-$ & 2.75E-01 & 1 & \\
\hline & 127 & & 77.00 & & $=$ & $2.62 E-02$ & 1 & \\
\hline & 128 & & 30.00 & & & $1.02 \mathrm{E}-02$ & 1 & \\
\hline & 129 & & 310.00 & & & $1.05 E-01$ & 1 & \\
\hline & 130 & & 41.00 & & & $1.39 \mathrm{E}-02$ & 9 & \\
\hline & 148 & & 86.00 & & & $2.92 E-02$ & 1 & \\
\hline & 149 & & 140.00 & & & 4.76E-02 & 1 & \\
\hline & 150 & & 29.00 & & & $9.86 \mathrm{E}-03$ & 1 & \\
\hline & 151 & & 310.00 & & & 1.05E-01 & 1 & \\
\hline & 152 & & 190.00 & & & $6.46 E-02$ & 1 & \\
\hline & 153 & & 290.00 & & & $9.86 E-02$ & 1 & \\
\hline & 154 & & 120,00 & & & $4.08 E-02$ & 1 & \\
\hline & 155 & & 170.00 & & & $5.78 E-02$ & 1 & \\
\hline & 156 & & 36.00 & & & $1.22 \mathrm{E}-02$ & $\overline{1}$ & \\
\hline & 157 & & 40.00 & & & 1.36E-02 & 1 & \\
\hline & 158 & & 140.00 & & & $4.76 E-02$ & 1 & \\
\hline
\end{tabular}


Table C-1. (cont.)

\begin{tabular}{|c|c|c|c|c|c|c|c|c|}
\hline Species & ID & Series & $\begin{array}{c}\text { U-238 } \\
(\mu \mathrm{g} / \mathrm{kg})\end{array}$ & $\begin{array}{c}\mathrm{U}-235 \\
(\mu \mathrm{g} / \mathrm{kg})\end{array}$ & $\begin{array}{l}\text { U-235/U-238 } \\
\text { Mass Ratio }\end{array}$ & $\begin{array}{l}\text { U-238 Conc } \\
\text { (pCi/g) }\end{array}$ & \#Individuals & \# Measurements \\
\hline & 159 & & 210.00 & & & $7.14 E-02$ & 23 & \\
\hline & 3079 & & 650.00 & & & 2.21E-01 & 4 & \\
\hline & 3144 & & 80.00 & & & $2.72 E-02$ & 6 & \\
\hline Ave. & & & $1.43 E+02$ & & & $4.86 E-02$ & 81 & 43 \\
\hline StDev & & & & & & $5.16 \mathrm{E}-02$ & & \\
\hline $\min$ & & & & & & $9.86 \mathrm{E}-03$ & & \\
\hline $\max$ & & & & & & $2.75 E-01$ & & \\
\hline Ave (no dups) & & & $1.46 E+02$ & & & $4.97 E-02$ & 78 & \\
\hline Stdev (no dups) & & & & & & $5.32 E-02$ & & \\
\hline $\min$ (no dups) & & & & & & $9.86 \mathrm{E}-03$ & & \\
\hline $\max$ (no dups) & & & & & & $2.75 \mathrm{E}-01$ & & \\
\hline & & & & & & & & \\
\hline \multicolumn{9}{|l|}{ Shad } \\
\hline & 101 & & 1100.00 & 35.00 & 0.0318 & 3.74E-01 & 33 & \\
\hline & 102 & & 2900.00 & 127.00 & 0.0438 & $9.86 \mathrm{E}-01$ & 31 & 2 \\
\hline Ave. & & & $2.00 E+03$ & & & $6.80 \mathrm{E}-01$ & 64 & \\
\hline StDev & & & & & & 4.33E-01 & & \\
\hline \multirow{2}{*}{\multicolumn{9}{|c|}{ Shrimp }} \\
\hline & & & & & & & & \\
\hline & 73 & & 50.00 & & & $1.70 \mathrm{E}-02$ & 4 & \\
\hline & 137 & & 140.00 & & & $4.76 \mathrm{E}-02$ & 5 & \\
\hline & $1 \widetilde{3}$ & & 410.00 & & & $1.39 \mathrm{E}-01$ & 21 & \\
\hline & 171 & & 620.00 & & & $2.11 E-01$ & 1 & \\
\hline Ave. & & & $3.05 E+02$ & & & $1.04 \mathrm{E}-01$ & 31 & 4 \\
\hline StDev & & & & & & $8.83 E-02$ & & \\
\hline \multirow{2}{*}{\multicolumn{9}{|c|}{ Silversides }} \\
\hline & & & & & & & & \\
\hline & 3076 & & 38.00 & & & $1.29 \mathrm{E}-02$ & 15 & \\
\hline & 3077 & & 42.00 & & & $1.43 E-02$ & 15 & \\
\hline & 3078 & & 60.00 & & & $2.04 \mathrm{E}-02$ & 12 & \\
\hline Ave. & & & $4.67 \mathrm{E}+01$ & & & $1.59 E-02$ & 42 & 4 \\
\hline StDev & & & & & & $3.98 \mathrm{E}-03$ & & \\
\hline & & & & & & & & \\
\hline \multicolumn{9}{|l|}{ Stone fily } \\
\hline & 139 & & 300.00 & & & $1.02 E-01$ & & \\
\hline & 165 & & 2100.00 & 150.00 & 0.0714 & 7.14E-01 & & \\
\hline Ave. & & & $1.20 E+03$ & & & 4.08E-01 & 2 & 2 \\
\hline StDev & & & & & & $4.33 \mathrm{E}-01$ & & \\
\hline \multirow{2}{*}{\multicolumn{9}{|c|}{ Stoneroller }} \\
\hline & & & & & & & & \\
\hline & 15 & $\overline{\mathrm{B}}$ & 170.0 & & 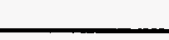 & $3.74 \mathrm{E}-02$ & & \\
\hline & 132 & $\bar{A}$ & 170.00 & & & $\frac{3.03 E-02}{5.78 E-02}$ & & \\
\hline & 133 & & 47.00 & & & $1.60 \mathrm{E}-02$ & & \\
\hline & 134 & & 300.00 & & & $1.02 \mathrm{E}-01$ & & \\
\hline & 141 & & 380.00 & & & 1.29E-01 & & \\
\hline & 165 & & 2100.00 & 150.00 & 0.0714 & 7.14E-01 & & \\
\hline Ave. & & & $4.57 E+02$ & & & $1.55 \mathrm{E}-01$ & 7 & 7 \\
\hline StDev & & & & & & $2.50 \mathrm{E}-01$ & & \\
\hline & & & & & & & & \\
\hline
\end{tabular}


Table C-1. (cont.)

\begin{tabular}{|c|c|c|c|c|c|c|c|c|}
\hline Species & ID & Series & $\begin{array}{c}U-238 \\
(\mu \mathrm{g} / \mathrm{kg})\end{array}$ & $\begin{array}{c}U-235 \\
(\mu \mathrm{g} / \mathrm{kg})\end{array}$ & $\begin{array}{l}\text { U-235/U-238 } \\
\text { Mass Ratio }\end{array}$ & $\begin{array}{l}\text { U-238 Conc } \\
\text { (pCilg) }\end{array}$ & \#Individuals & \#Measurements \\
\hline \multicolumn{9}{|c|}{ Perch, no duplicates } \\
\hline Source & ID & Series & $\begin{array}{c}\mathrm{U}-238 \\
(\mu \mathrm{g} / \mathrm{kg})\end{array}$ & $\begin{array}{c}\mathrm{U}-235 \\
(\mu \mathrm{g} / \mathrm{kg})\end{array}$ & $\begin{array}{l}\text { U-235/U-238 } \\
\text { Mass Ratio }\end{array}$ & $\begin{array}{l}\text { U-238 Conc } \\
\text { (pCi/g) }\end{array}$ & \# Individuals & \\
\hline APG & 2 & & 90.00 & & & $3.06 \mathrm{E}-02$ & 1 & \\
\hline APG & 4 & & 49.00 & & & $1.67 \mathrm{E}-02$ & 1 & \\
\hline APG & 6 & & 110.00 & & & $3.74 E-02$ & 1 & \\
\hline APG & 7 & & 100.00 & & & $3.40 \mathrm{E}-02$ & 1 & \\
\hline APG & 8 & & 330.00 & & & 1.12E-01 & 1 & \\
\hline APG & 10 & & 160.00 & & & $5.44 E-02$ & 1 & \\
\hline APG & 12 & & 74.00 & & & $2.52 E-02$ & 1 & \\
\hline APG & 14 & & 66.00 & & & $2.24 E-02$ & 1 & \\
\hline APG & 16 & & 58.00 & & & 1.97E-02 & 1 & \\
\hline APG & 18 & & 28.00 & & & $9.52 \mathrm{E}-03$ & 1 & \\
\hline APG & 21 & & 100.00 & & & $3.40 E-02$ & 1 & \\
\hline APG & 22 & & 260.00 & & & $8.84 E-02$ & 1 & \\
\hline APG & 24 & & 300.00 & & & $1.02 E-01$ & 1 & \\
\hline APG & 26 & & 130.00 & & & $4.42 E-02$ & 1 & \\
\hline APG & 20 & & 90.00 & & & $3.06 E-02$ & 1 & \\
\hline APG & 31 & & 150.00 & & & $5.10 E-02$ & 1 & \\
\hline APG & 33 & & 110.00 & & & $3.74 E-02$ & 1 & \\
\hline APG & 44 & & 210.00 & & & 7.14E-02 & 1 & \\
\hline APG & 46 & & 120.00 & & & $4.08 E-02$ & 1 & \\
\hline APG & 47 & & 94.00 & & & $3.20 \mathrm{E}-02$ & 1 & \\
\hline APG & 48 & & 170.00 & & & $5.78 E-02$ & 1 & \\
\hline APG & 50 & & 92.00 & & & $3.13 E-02$ & 1 & \\
\hline APG & 51 & & 110.00 & & & $3.74 E-02$ & 1 & \\
\hline$\overline{A P G}$ & 54 & & 890.00 & 17.00 & 0.0191 & $3.03 E-01$ & 3 & \\
\hline$\overline{A P G}$ & 56 & & 160.00 & & & $5.44 E-02$ & 1 & \\
\hline APG & 57 & & 78.00 & & & $2.65 \mathrm{E}-02$ & 1 & \\
\hline APG & 59 & & 59.00 & & & 2.01E-02 & 1 & \\
\hline APG & 60 & & 250.00 & & & $8.50 \mathrm{E}-02$ & 1 & \\
\hline APG & 61 & & 150.00 & & & $5.10 E-02$ & 1 & \\
\hline APG & 62 & & 170.00 & & & $5.78 E-02$ & 1 & \\
\hline APG & 6 & & 150.00 & & & $5.10 E-02$ & 1 & \\
\hline APG & 65 & & 220.00 & & & $7.48 \mathrm{E}-02$ & 1 & \\
\hline APG & 67 & & 110.00 & & & 3.74E-02 & 1 & \\
\hline APG & 68 & & 67.00 & & & $228 \mathrm{E}-02$ & 1 & \\
\hline APG & 69 & & 220.00 & & & $7.48 \mathrm{E}-02$ & 1 & \\
\hline APG & 71 & & 69.00 & & & $2.35 \mathrm{E}-02$ & 1 & \\
\hline APG & 74 & & 66.00 & & & $2.24 \mathrm{E}-02$ & 1 & \\
\hline APG & 76 & & 66.00 & & & $2.24 \mathrm{E}-02$ & 1 & \\
\hline APG & 78 & & 60.00 & & & $2.04 \mathrm{E}-02$ & 1 & \\
\hline APG & 85 & & 150.00 & & & $5.10 \mathrm{E}-02$ & 1 & \\
\hline APG & 87 & & 39.00 & & & $1.33 \mathrm{E}-02$ & 1 & \\
\hline APG & 89 & & 40.00 & & & $1.36 \mathrm{E}-02$ & 1 & \\
\hline APG & 91 & & 140.00 & & & $4.76 \mathrm{E}-02$ & 1 & \\
\hline APG & 92 & & 75.00 & & & $2.55 \mathrm{E}-02$ & 1 & \\
\hline APG & 94 & & 56.00 & & & $1.90 \mathrm{E}-02$ & 1 & \\
\hline APG & 96 & & 80.00 & & & $2.72 E-02$ & 1 & \\
\hline APG & 97 & & 79.00 & & & $2.69 E-02$ & 1 & \\
\hline APG & 98 & & 140.00 & & & $4.76 E-02$ & 1 & \\
\hline
\end{tabular}


Table C-1. (cont.)

\begin{tabular}{|c|c|c|c|c|c|c|c|c|}
\hline Species & ID & Series & $\begin{array}{c}\text { U-238 } \\
(\mu \mathrm{g} / \mathrm{kg})\end{array}$ & $\begin{array}{c}\text { U-235 } \\
(\mu \mathrm{g} / \mathrm{kg})\end{array}$ & $\begin{array}{l}\text { U-235/U-238 } \\
\text { Mass Ratio }\end{array}$ & $\begin{array}{l}\text { U-238 Conc } \\
\text { (pCilg) }\end{array}$ & \# Individuals & \# Measurements \\
\hline APG & 99 & & 130.00 & & & 4.42E-02 & 1 & \\
\hline APG & 100 & & 110.00 & & & $3.74 \mathrm{E}-02$ & 1 & \\
\hline APG & 103 & & 110.00 & & & 3.74E-02 & 1 & \\
\hline APG & 3019 & & 94.00 & & & $3.20 \mathrm{E}-02$ & 10 & \\
\hline APG & 3033 & & 40.00 & & & 1.36E-02 & 10 & \\
\hline$\overline{A P G}$ & 3070 & & 110.00 & & & $3.74 \mathrm{E}-02$ & 10 & \\
\hline$\overline{A P G}$ & 3071 & & 310.00 & & & 1.05E-01 & 7 & \\
\hline$\overline{A P G}$ & 3073 & & 71.00 & & & 2.41E-02 & 10 & \\
\hline$\overline{A P G}$ & 3137 & & 270.00 & & & $9.18 \mathrm{E}-02$ & 1 & \\
\hline$\overline{A P G}$ & 3145 & & 99.00 & & & 3.37E-02 & 1 & \\
\hline APG & 3147 & & 42.00 & & & 1.43E-02 & $\overline{1}$ & \\
\hline$\overline{A P G}$ & 3148 & & 58.00 & & & 1.97E-02 & 1 & \\
\hline$\overline{\mathrm{APG}}$ & 3149 & & 43.00 & & & $1.46 \mathrm{E}-02$ & 1 & \\
\hline \multirow[t]{12}{*}{ APG } & 3150 & & 18.00 & & & 6.12E-03 & 1 & \\
\hline & & & & & & & & \\
\hline & & & & & Statistics & & & \\
\hline & & & & & Ave. & 4.44E-02 & & \\
\hline & & & & & Median & $3.40 \mathrm{E}-02$ & & \\
\hline & & & & & Mode & $3.74 E-02$ & & \\
\hline & & & & & $\begin{array}{l}\text { Standard } \\
\text { Deviation }\end{array}$ & 4.14E-02 & & \\
\hline & & & & & Range & $2.96 E-01$ & & \\
\hline & & & & & Minimum & $6.12 E-03$ & & \\
\hline & & & & & Maximum & 3.03E-01 & & \\
\hline & & & & & Sum & $2.75 E+00$ & & \\
\hline & & & & & \begin{tabular}{|r|} 
Count (\# \\
measurement \\
s)
\end{tabular} & $\overline{2}$ & & \\
\hline
\end{tabular}


Table C-2. Aquatic data for APG by compartment identification. Compartments $\mathrm{C}_{1}$ through $\mathrm{C}_{4}$ not sampled at APG.

\begin{tabular}{|c|c|c|c|c|c|c|c|c|}
\hline Species & ID & Series & $\mathrm{U}-238(\mu \mathrm{g} / \mathrm{kg})$ & $U-235(\mu \mathrm{g} / \mathrm{kg})$ & $\begin{array}{l}\text { U-235T-238 } \\
\text { Mass Ratio }\end{array}$ & $\begin{array}{l}\text { U-238 Conc } \\
\text { (pCi/g) }\end{array}$ & \#Individuals & \# Measurements \\
\hline \multicolumn{9}{|l|}{ Compartment $\mathrm{C}_{5}$} \\
\hline \multicolumn{9}{|l|}{ Belastoma } \\
\hline & 138 & & 280.00 & & & $9.52 E-02$ & 2 & 1 \\
\hline & & & & & & & & \\
\hline \multicolumn{9}{|l|}{ Shrimp } \\
\hline & 73 & & 50.00 & & & $1.70 \mathrm{E}-02$ & 4 & \\
\hline & 137 & & 140.00 & & & $4.76 E-02$ & 5 & \\
\hline & $\overline{163}$ & & 410.00 & & & $1.39 E-01$ & 21 & \\
\hline & $\overline{171}$ & & 620.00 & & & 2.11E-01 & 1 & \\
\hline & & & & & & & & \\
\hline \multicolumn{9}{|l|}{ Stone fly } \\
\hline & 139 & & 300.00 & & & $1.02 E-01$ & & \\
\hline & 165 & & 2100.00 & 150.00 & 0.0714 & 7.14E-01 & & \\
\hline & & & & & & & & \\
\hline & & & & & Ave & 1.89E-01 & & \\
\hline & & & & & Stdev & $240 E-01$ & & \\
\hline & & & & & Min & 1.70E-02 & & \\
\hline & & & & & Max & 7.14E-01 & & \\
\hline & & & & & Count & 7 & & \\
\hline & & & & & $95 \% \mathrm{Cl}$ & 1.78E-01 & & \\
\hline & & & & & & & & \\
\hline \multicolumn{9}{|l|}{ Compartment $C_{6}$} \\
\hline \multicolumn{9}{|l|}{ Clam } \\
\hline & 3035 & & 120.00 & & & $4.08 \mathrm{E}-02$ & 1 & \\
\hline & 3059 & & 250.00 & & & $8.50 \mathrm{E}-02$ & 2 & \\
\hline & 3061 & & 360.00 & & & $1.22 \mathrm{E}-01$ & 2 & \\
\hline & 3062 & & 300.00 & & & $1.02 E-01$ & 1 & \\
\hline & 82 & $\bar{B}$ & 10.00 & & & $3.40 \mathrm{E}-03$ & 2 & \\
\hline & 82 & $\bar{A}$ & 30.00 & & & $1.02 \mathrm{E}-02$ & 2 & \\
\hline & & & & & & & & \\
\hline & & & & & Ave & $6.06 E-02$ & & \\
\hline & & & & & Stdev & 4.97E-02 & & \\
\hline & & & & & Min & $3.40 \mathrm{E}-03$ & & \\
\hline & & & & & Max & 1.22E-01 & & \\
\hline & & & & & Count & $6.00 E+00$ & & \\
\hline & & & & & $95 \% \mathrm{Cl}$ & $3.97 E-02$ & & \\
\hline & & & & & & & & \\
\hline \multicolumn{9}{|l|}{ Compartment $C_{7}$} \\
\hline \multicolumn{9}{|l|}{ Blue Crab } \\
\hline & 3088 & 1-3, hepato & 69.00 & & & $235 \mathrm{E}-02$ & 3 & \\
\hline & 3094 & $1-3$, muscle & 66.00 & & & $224 \mathrm{E}-02$ & 3 & \\
\hline & 3130 & $1-2$, muscle & 32.00 & & & $1.09 \mathrm{E}-02$ & 2 & \\
\hline & 83 & & 86.00 & & & $2.92 E-02$ & 1 & \\
\hline & 173 & & 200.00 & -1.75 & & $6.80 \mathrm{E}-02$ & 1 & \\
\hline
\end{tabular}


Table C-2. (cont.)

\begin{tabular}{|c|c|c|c|c|c|c|c|c|}
\hline Species & ID & Series & $\mathrm{U}-238(\mu \mathrm{g} / \mathrm{kg})$ & $\mathrm{U}-235(\mu \mathrm{g} / \mathrm{kg})$ & $\begin{array}{l}\text { U-235/U-238 } \\
\text { Mass Ratio } \\
\end{array}$ & $\begin{array}{c}\text { U-238 Conc } \\
\text { (pCi/g) }\end{array}$ & \# Individuals & \# Measurements \\
\hline & 174 & A & 89.00 & & & 3.03E-02 & 1 & \\
\hline & 174 & $\bar{B}$ & 130.00 & & & $4.42 E-02$ & 1 & \\
\hline & & & & & & & & \\
\hline \multicolumn{9}{|l|}{ Bullhead } \\
\hline & 3103 & & 380.00 & & & $1.29 \mathrm{E}-01$ & 1 & \\
\hline & 143 & & 180.00 & & & $6.12 E-02$ & 2 & \\
\hline & 144 & & 280.00 & & & $9.52 E-02$ & 2 & \\
\hline & 145 & & 250.00 & & & $8.50 E-02$ & 1 & \\
\hline & & & & & & & & \\
\hline \multicolumn{9}{|l|}{ Carp } \\
\hline & 3001 & $\# 2$, BONES & 400.00 & & & $1.36 \mathrm{E}-01$ & 1 & \\
\hline & 3003 & \#1 LIVER & 200.00 & & & $6.80 \mathrm{E}-02$ & 1 & \\
\hline & 3004 & \#2 GUT & 24.00 & & & $8.16 E-03$ & 1 & \\
\hline & 3005 & \#1 GUT & 62.00 & & & $2.11 \mathrm{E}-02$ & 1 & \\
\hline & 3008 & \#2 LIVER & 490.00 & & & $1.67 E-01$ & 1 & \\
\hline & 3018 & \#2 FILLETS & 14.00 & & & $4.76 E-03$ & 1 & \\
\hline & & & & & & & & \\
\hline \multicolumn{9}{|l|}{ Catfish } \\
\hline & 3080 & liver & -1.00 & & & $-3.40 E-04$ & 1 & \\
\hline & 3082 & fillets & 230.00 & & & $7.82 E-02$ & 1 & 2 \\
\hline & & & & & & & & \\
\hline \multicolumn{9}{|l|}{ Crawtish } \\
\hline & 164 & & 310.00 & & & $1.05 E-01$ & 1 & 1 \\
\hline \multirow{2}{*}{\multicolumn{9}{|c|}{ Darter }} \\
\hline & & & & & & & & \\
\hline & 104 & & 31.00 & & & $1.05 E-02$ & 1 & 1 \\
\hline & & & & & & & & \\
\hline \multicolumn{9}{|l|}{ Hogchocker } \\
\hline & 3044 & & 160.00 & & & $5.44 E-02$ & 4 & \\
\hline & 3045 & & 160.00 & & & $5.44 \mathrm{E}-02$ & 1 & \\
\hline & 27 & & 26.00 & & & $8.84 E-03$ & 1 & \\
\hline & 38 & & 160.00 & & & $5.44 E-02$ & 1 & \\
\hline & 41 & & 180.00 & & & $6.12 E-02$ & 1 & \\
\hline & 72 & & 61.00 & & & $2.07 E-02$ & 1 & \\
\hline & 84 & & 110.00 & & & $3.74 \mathrm{E}-02$ & 1 & \\
\hline \multirow{2}{*}{\multicolumn{9}{|c|}{ Stoneroller }} \\
\hline & & & & & & & & \\
\hline & 131 & & 110.00 & & & 3.74E-02 & & \\
\hline & 132 & $\mathrm{~B}$ & 89.00 & & & $3.03 E-02$ & & \\
\hline & 132 & $\bar{A}$ & 170.00 & & & $5.78 E-02$ & & \\
\hline & 133 & & 47.00 & & & $1.60 \mathrm{E}-02$ & & \\
\hline & 134 & & 300.00 & & & $1.02 E-01$ & & \\
\hline & 141 & & 380.00 & & & 1.29E-01 & & \\
\hline & 165 & & 2100.00 & 150.00 & 0.0714 & 7.14E-01 & & \\
\hline & & & & & & & & \\
\hline
\end{tabular}


Table C-2. (cont.)

\begin{tabular}{|c|c|c|c|c|c|c|c|c|}
\hline Species & ID & Series & j-238 $(\mu \mathrm{g} / \mathrm{kg})$ & $\mathrm{U}-235(\mu \mathrm{g} / \mathrm{kg})$ & \begin{tabular}{|l|} 
U-235/U-238 \\
Mass Ratio
\end{tabular} & $\begin{array}{c}\text { U-238 Conc } \\
\text { (pCi/g) }\end{array}$ & \# Individuals & \# Measurements \\
\hline \multicolumn{9}{|l|}{ Tadpoles } \\
\hline & 170 & & 510.00 & & & $1.73 E-01$ & 1 & \\
\hline & 3093 & & 710.00 & 56.00 & 0.0789 & $241 E-01$ & 2 & \\
\hline & $\overline{3102}$ & & 690.00 & & & $2.35 E-01$ & 2 & \\
\hline & 3111 & & 240.00 & & & $8.16 \mathrm{E}-02$ & 1 & \\
\hline & $\overline{3154}$ & & 440.00 & & & $1.50 E-01$ & 12 & \\
\hline & & & & & & & & \\
\hline & & & & & Ave & $8.64 E-02$ & & \\
\hline & & & & & Stdev & $1.18 E-01$ & & \\
\hline & & & & & $\overline{\text { Min }}$ & $-3.40 E-04$ & & \\
\hline & & & & & $\operatorname{Max}$ & 7.14E-01 & & \\
\hline & & & & & Count & 40 & & \\
\hline & & & & & $95 \% \mathrm{Cl}$ & $3.67 E-02$ & & \\
\hline & & & & & & & & \\
\hline \multicolumn{9}{|l|}{ Compartment $\mathrm{C}_{\mathrm{B}}$} \\
\hline \multicolumn{9}{|l|}{ Anchovy } \\
\hline & 3069 & & 55.00 & & & $1.87 E-02$ & 2 & \\
\hline & 34 & & 260.00 & & & $8.84 E-02$ & $\overline{21}$ & \\
\hline & 53 & & 810.00 & 10.00 & 0.0123 & $275 E-01$ & 48 & \\
\hline & 80 & & 100.00 & & & $3.40 E-02$ & 20 & \\
\hline & 81 & & 59.00 & & & $2.01 E-02$ & 6 & \\
\hline & $\overline{112}$ & & 15.00 & & & $5.10 E-03$ & 11 & \\
\hline & 113 & & 44.00 & & & $1.50 E-02$ & 18 & \\
\hline & & & & & & & & \\
\hline \multicolumn{9}{|l|}{ Chub } \\
\hline & 160 & & 50.00 & & & $1.70 \mathrm{E}-02$ & $\overline{1}$ & \\
\hline & 161 & & 34.00 & & & $1.16 E-02$ & 1 & \\
\hline & & & & & & & & \\
\hline \multicolumn{9}{|l|}{ Mosquito fish } \\
\hline & 162 & & 25.00 & & & $8.50 \mathrm{E}-03$ & 3 & 1 \\
\hline & & & & & & & & \\
\hline \multicolumn{9}{|l|}{ Sunfish } \\
\hline & 39 & & 39.00 & & & $1.33 \mathrm{E}-02$ & 1 & \\
\hline & 40 & & 94.00 & & & $3.20 \mathrm{E}-02$ & 1 & \\
\hline & 105 & $\bar{A}$ & 43.00 & & & $1.46 \mathrm{E}-02$ & 1 & \\
\hline & 105 & $\bar{B}$ & 78.00 & & & $2.65 E-02$ & 1 & \\
\hline & 106 & & 160.00 & & & $5.44 E-02$ & 1 & \\
\hline & 107 & & 58.00 & & & $1.97 E-02$ & 1 & \\
\hline & 108 & $\bar{A}$ & 130.00 & & & $4.42 E-02$ & 1 & \\
\hline & 108 & $\bar{B}$ & 160.00 & & & $5.44 E-02$ & 1 & \\
\hline & 109 & & 87.00 & & & $2.96 E-02$ & 1 & \\
\hline & 110 & & 59.00 & & & 2.01E-02 & 1 & \\
\hline & 111 & & 130.00 & & & $4.42 E-02$ & 1 & \\
\hline & 114 & & 140.00 & & & $4.76 E-02$ & 1 & \\
\hline
\end{tabular}


Table C-2. (cont.)

\begin{tabular}{|c|c|c|c|c|c|c|c|c|}
\hline Species & ID & Series & $\mathrm{U}-238(\mu \mathrm{g} / \mathrm{kg})$ & $\mathrm{U}-235(\mu \mathrm{g} / \mathrm{kg})$ & $\begin{array}{l}\text { U-235U-238 } \\
\text { Mass Ratio }\end{array}$ & $\begin{array}{c}\text { U-238 Conc } \\
\text { (pCilg) }\end{array}$ & \# Individuals & \# Measurements \\
\hline & 115 & & 36.00 & & & $1.22 \mathrm{E}-02$ & 1 & \\
\hline & 116 & & 49.00 & & & $1.67 \mathrm{E}-02$ & 1 & \\
\hline & 117 & & 93.00 & & & $3.16 E-02$ & 1 & \\
\hline & 118 & & 38.00 & & & $1.29 \mathrm{E}-02$ & 1 & \\
\hline & 119 & & 59.00 & & & $2.01 \mathrm{E}-02$ & 1 & \\
\hline & 120 & $\bar{B}$ & 69.00 & & & $2.35 E-02$ & 1 & \\
\hline & 120 & $\bar{A}$ & 170.00 & & & $5.78 \mathrm{E}-02$ & 1 & \\
\hline & 121 & & 130.00 & & & $4.42 E-02$ & 1 & \\
\hline & 122 & & 170.00 & & & $5.78 E-02$ & 1 & \\
\hline & 123 & & 170.00 & & & $5.78 E-02$ & 1 & \\
\hline & 124 & & 31.00 & & & $1.05 E-02$ & 1 & \\
\hline & 125 & & 200.00 & & & $6.80 E-02$ & 1 & \\
\hline & 126 & & 810.00 & 5.50 & 0.0068 & $2.75 E-01$ & 1 & \\
\hline & 127 & & 77.00 & & & $2.62 E-02$ & 1 & \\
\hline & 128 & & 30.00 & & & $1.02 E-02$ & 1 & \\
\hline & 129 & & 310.00 & & & $1.05 E-01$ & 1 & \\
\hline & 130 & & 41.00 & & & $1.39 E-02$ & 9 & \\
\hline & 148 & & 86.00 & & & $2.92 E-02$ & 1 & \\
\hline & 149 & & 140.00 & & & $4.76 E-02$ & 1 & \\
\hline & 150 & & 29.00 & & & $9.86 E-03$ & 1 & \\
\hline & 151 & & 310.00 & & & 1.05E-01 & 1 & \\
\hline & 152 & & 190.00 & & & $6.46 E-02$ & 1 & \\
\hline & 153 & & 290.00 & & & $9.86 \mathrm{E}-02$ & 1 & \\
\hline & 154 & & 120.00 & & & $4.08 \mathrm{E}-02$ & 1 & \\
\hline & 155 & & 170.00 & & & $5.78 E-02$ & 1 & \\
\hline & 156 & & 36.00 & & & $1.22 E-02$ & 1 & \\
\hline & 157 & & 40.00 & & & $1.36 \mathrm{E}-02$ & 1 & \\
\hline & 158 & & 140.00 & & & $4.76 E-02$ & 1 & \\
\hline & 159 & & 210.00 & & & $7.14 E-02$ & 23 & \\
\hline & 3079 & & 650.00 & & & $2.21 \mathrm{E}-01$ & 4 & \\
\hline & 3144 & & 80.00 & & & $2.72 E-02$ & 6 & \\
\hline \multirow{2}{*}{\multicolumn{9}{|c|}{ Shad }} \\
\hline & & & & & & & & \\
\hline & 101 & & 1100.00 & 35.00 & 0.0318 & $3.74 E-01$ & 33 & \\
\hline & 102 & & 2900.00 & 127.00 & 0.0438 & $9.86 E-01$ & 31 & 2 \\
\hline \multicolumn{9}{|l|}{ Silversides } \\
\hline & 3076 & & 38.00 & & & $1.29 E-02$ & 15 & \\
\hline & 3077 & & 42.00 & & & $1.43 E-02$ & 15 & \\
\hline & 3078 & & 60.00 & & & $2.04 E-02$ & 12 & \\
\hline \multicolumn{9}{|l|}{ White Perch } \\
\hline & 2 & & 90.00 & & & $3.06 E-02$ & 1 & \\
\hline & 4 & & 49.00 & & & $1.67 E-02$ & 1 & \\
\hline
\end{tabular}


Table C-2. (cont.)

\begin{tabular}{|c|c|c|c|c|c|c|c|c|}
\hline Species & ID & Series & $\mathrm{U}-238(\mu \mathrm{g} / \mathrm{kg})$ & $\mathrm{U}-235(\mu \mathrm{g} / \mathrm{kg})$ & $\begin{array}{l}\text { U-235/U-238 } \\
\text { Mass Ratio }\end{array}$ & $\begin{array}{l}\text { U-238 Cone } \\
\text { (pCi/g) }\end{array}$ & \# Individuals & \# Measúrements \\
\hline & & & & & & & & \\
\hline & 6 & & 110.00 & & & $3.74 E-02$ & 1 & \\
\hline & 7 & & 100.00 & & & $3.40 E-02$ & 1 & \\
\hline & 8 & & 330.00 & & & 1.12E-01 & 1 & \\
\hline & 10 & & 160.00 & & & $5.44 \mathrm{E}-02$ & 1 & \\
\hline & 12 & & 74.00 & & & $2.52 E-02$ & 1 & \\
\hline & 14 & & 66.00 & & & $2.24 \mathrm{E}-02$ & 1 & \\
\hline & 16 & & 58.00 & & & $1.97 E-02$ & 1 & \\
\hline & 18 & & 28.00 & & & $9.52 \mathrm{E}-03$ & 1 & \\
\hline & 21 & & 100.00 & & & $3.40 E-02$ & 1 & \\
\hline & $\overline{22}$ & & 260.00 & & & $8.84 \mathrm{E}-02$ & 1 & \\
\hline & 24 & & 300.00 & & & $1.02 E-01$ & 1 & \\
\hline & 26 & & 130.00 & & & $4.42 E-02$ & 1 & \\
\hline & 29 & & 90.00 & & & $3.06 \mathrm{E}-02$ & 1 & \\
\hline & $\overline{31}$ & & 150.00 & & & $5.10 E-02$ & 1 & \\
\hline & 33 & & 110.00 & & & $3.74 \mathrm{E}-02$ & 1 & \\
\hline & 44 & & 210.00 & & & $7.14 E-02$ & 1 & \\
\hline & 46 & & 120.00 & & & $4.08 E-02$ & 1 & \\
\hline & 47 & & 94.00 & & & $3.20 \mathrm{E}-02$ & 1 & \\
\hline & 48 & & 170.00 & & & $5.78 \mathrm{E}-02$ & 1 & \\
\hline & 50 & & 9200 & & & $3.13 E-02$ & 1 & \\
\hline & $\overline{51}$ & & 110.00 & & & $3.74 \mathrm{E}-02$ & 1 & \\
\hline & 54 & & 890.00 & 17.00 & 0.0191 & 3.03E-01 & $\overline{3}$ & \\
\hline & 55 & & 160.00 & & & $5.44 E-02$ & 1 & \\
\hline & 57 & & 78.00 & & & $2.65 E-02$ & 1 & \\
\hline & 59 & & 59.00 & & & $2.01 \mathrm{E}-02$ & 1 & \\
\hline & 60 & & 250.00 & & & $8.50 E-02$ & 1 & \\
\hline & $\overline{61}$ & & 150.00 & & & $5.10 E-02$ & 1 & \\
\hline & $\overline{62}$ & & 170.00 & & & $5.78 E-02$ & 1 & \\
\hline & $\overline{63}$ & & 150.00 & & & $5.10 \mathrm{E}-02$ & 1 & \\
\hline & 65 & & 220.00 & & & $7.48 E-02$ & 1 & \\
\hline & 67 & & 110.00 & & & $3.74 E-02$ & 1 & \\
\hline & $\overline{68}$ & & 67.00 & & & $2.28 E-02$ & 1 & \\
\hline & 69 & & 220.00 & & & $7.48 E-02$ & 1 & \\
\hline & 71 & & 69.00 & & & $235 E-02$ & 1 & \\
\hline & 74 & & 66.00 & & & $2.24 \mathrm{E}-02$ & 1 & \\
\hline & 76 & & 66.00 & & & $2.24 E-02$ & 1 & \\
\hline & 78 & & 60.00 & & & $2.04 E-02$ & 1 & \\
\hline & 85 & & 150.00 & & & $5.10 \mathrm{E}-02$ & 1 & \\
\hline & 87 & & 39.00 & & & $1.33 E-02$ & 1 & \\
\hline & 89 & & 40.00 & & & $1.36 \mathrm{E}-02$ & 1 & \\
\hline & 9 & & 140.00 & & & $4.76 E-02$ & 1 & \\
\hline & 92 & & 75.00 & & & $2.55 \mathrm{E}-02$ & 1 & \\
\hline & 94 & & 56.00 & & & $1.90 \mathrm{E}-02$ & 1 & \\
\hline
\end{tabular}


Table C-2. (cont.)

\begin{tabular}{|c|c|c|c|c|c|c|c|c|}
\hline Species & ID & Series & $\mathrm{U}-238(\mu \mathrm{g} / \mathrm{kg})$ & $U-235(\mu \mathrm{g} / \mathrm{kg})$ & \begin{tabular}{|l|} 
U-235/U-238 \\
Mass Ratio
\end{tabular} & $\begin{array}{l}\text { U-238 Conc } \\
\text { (pCi/g) }\end{array}$ & \# Individuals & \# Measurements \\
\hline & & & & & & & & \\
\hline & 96 & & 80.00 & & & $2.72 E-02$ & 1 & \\
\hline & 97 & & 79.00 & & & $2.69 E-02$ & 1 & \\
\hline & 98 & & 140.00 & & & $4.76 E-02$ & 1 & \\
\hline & 9 & & 130.00 & & & $4.42 E-02$ & 1 & \\
\hline & 100 & & 110.00 & & & $3.74 E-02$ & 1 & \\
\hline & 103 & & 110.00 & & & $3.74 E-02$ & 1 & \\
\hline & 3019 & & 94.00 & & & $3.20 \mathrm{E}-02$ & 10 & \\
\hline & 3033 & & 40.00 & & & $1.36 E-02$ & 10 & \\
\hline & 3070 & & 110.00 & & & $3.74 \mathrm{E}-02$ & 10 & \\
\hline & 3071 & & 310.00 & & & $1.05 E-01$ & 7 & \\
\hline & 3073 & & 71.00 & & & $2.41 E-02$ & 10 & \\
\hline & 3137 & & 270.00 & & & $9.18 E-02$ & 1 & \\
\hline & $\overline{3145}$ & & 99.00 & & & $3.37 \mathrm{E}-02$ & 1 & \\
\hline & 3147 & & 4200 & & & $1.43 E-02$ & 1 & \\
\hline & 3448 & & 58.00 & & & $1.97 \mathrm{E}-02$ & 1 & \\
\hline & 3149 & & 43.00 & & & $1.46 E-02$ & 1 & \\
\hline & 3150 & & 18.00 & & & $6.12 E-03$ & 1 & \\
\hline \multirow{2}{*}{\multicolumn{9}{|c|}{ Yellow Perch }} \\
\hline & & & & & & & & \\
\hline & 11 & $\bar{A}$ & 38.00 & & & $1.29 \mathrm{E}-02$ & 1 & \\
\hline & 11 & $\bar{B}$ & 630.00 & & & $2.14 \mathrm{E}-01$ & 1 & \\
\hline & 35 & $\bar{A}$ & 83.00 & & & $2.82 E-02$ & 1 & \\
\hline & 35 & B & 98.00 & & & $3.33 E-02$ & 1 & \\
\hline & 42 & & 92.00 & & & $3.13 E-02$ & 1 & \\
\hline & 125 & liver & 200.00 & & & $6.80 E-02$ & 2 & \\
\hline & 126 & carcass & 810.00 & 5.50 & 0.0068 & $2.75 E-01$ & 2 & \\
\hline & 127 & fillets & 77.00 & & & $2.62 \mathrm{E}-02$ & 2 & \\
\hline & 128 & kidney & 30.00 & & & $1.02 E-02$ & 2 & \\
\hline & & & & & & & & \\
\hline & & & & & Ave & $5.77 \mathrm{E}-02$ & & \\
\hline & & & & & Stdev & $1.02 \mathrm{E}-01$ & & \\
\hline & & & & & Min & $5.10 \mathrm{E}-03$ & & \\
\hline & & & & & Max & $9.86 E-01$ & & \\
\hline & & & & & Count & 129 & & \\
\hline & & & & & $95 \% \mathrm{Cl}$ & $1.77 E-02$ & & \\
\hline & & & & & & & & \\
\hline & & & & & & & & \\
\hline
\end{tabular}


Table C-2. (cont.)

\begin{tabular}{|c|c|c|c|c|c|c|c|c|}
\hline Species & ID & Series & $\mathrm{U}-238(\mu \mathrm{g} / \mathrm{kg})$ & $\mathrm{U}-2335(\mu \mathrm{g} / \mathrm{kg})$ & $\begin{array}{l}\text { U-235万U-238 } \\
\text { Mass Ratio }\end{array}$ & $\begin{array}{c}\text { U-238 Conc } \\
\text { (pCilg) }\end{array}$ & \#Individuals & \# Measurements \\
\hline \multicolumn{9}{|c|}{ Compartment $C_{9}$} \\
\hline \multicolumn{9}{|c|}{\begin{tabular}{l|l} 
Pickerel & \\
\end{tabular}} \\
\hline & 3118 & & 7.50 & & & $2.55 E-03$ & 1 & \\
\hline & 3120 & & 15.00 & & & $5.10 E-03$ & 1 & \\
\hline & & & $1.13 E+01$ & & & $3.83 E-03$ & 2 & 2 \\
\hline & & & & & & & & \\
\hline \multicolumn{9}{|l|}{ White Perch } \\
\hline & $\overline{2}$ & & 90.00 & & & $3.06 E-02$ & 1 & \\
\hline & $\overline{4}$ & & 49.00 & & & $1.67 E-02$ & 1 & \\
\hline & 6 & & 110.00 & & & 3.74E-02 & 1 & \\
\hline & 7 & & 100.00 & & & $3.40 E-02$ & 1 & \\
\hline & 8 & & 330.00 & & & $1.12 E-01$ & 1 & \\
\hline & 10 & & 160.00 & & & $5.44 E-02$ & 1 & \\
\hline & 12 & & 74.00 & & & $2.52 E-02$ & 1 & \\
\hline & 14 & & 66.00 & & & $2.24 E-02$ & $\overline{1}$ & \\
\hline & $\overline{16}$ & & 58.00 & & & $1.97 E-02$ & 1 & \\
\hline & 18 & & 28.00 & & & $9.52 \mathrm{E}-03$ & 1 & \\
\hline & 21 & & 100.00 & & & $3.40 \mathrm{E}-02$ & 1 & \\
\hline & 22 & & 260.00 & & & $8.84 E-02$ & $T$ & \\
\hline & 24 & & 300.00 & & & $1.02 E-01$ & 1 & \\
\hline & 26 & & 130.00 & & & $4.42 E-02$ & 1 & \\
\hline & 29 & & 90.00 & & & $3.06 E-02$ & 1 & \\
\hline & 31 & & 150.00 & & & $5.10 E-02$ & 1 & \\
\hline & 33 & & 110.00 & & & $3.74 E-02$ & 1 & \\
\hline & $\overline{44}$ & & 210.00 & & & $7.14 E-02$ & 1 & \\
\hline & 46 & & 120.00 & & & $4.08 \mathrm{E}-02$ & $T$ & \\
\hline & 47 & & 94.00 & & & $3.20 \mathrm{E}-02$ & 1 & \\
\hline & 48 & & 170.00 & & & $5.78 \mathrm{E}-02$ & 1 & \\
\hline & 50 & & 9200 & & & $3.13 \mathrm{E}-02$ & 1 & \\
\hline & 51 & & 110.00 & & & 3.74E-02 & 1 & \\
\hline & 54 & & 890.00 & 17.00 & 0.0191 & 3.03E-01 & $\overline{3}$ & \\
\hline & 5 & & 160.00 & & & $5.44 E-02$ & 1 & \\
\hline & 57 & & 78.00 & & & 2 .65E-02 & 1 & \\
\hline & 59 & & 59.00 & & & $2.01 E-02$ & 1 & \\
\hline & 6 & & 250.00 & & & $8.50 E-02$ & 1 & \\
\hline & 61 & & 150.00 & & & $5.10 E-02$ & 1 & \\
\hline & ⿷匚 & & 170.00 & & & $5.78 E-02$ & 1 & \\
\hline & $\overline{63}$ & & 150.00 & & & $5.10 \mathrm{E}-02$ & 1 & \\
\hline
\end{tabular}


Table C-2. (cont.)

\begin{tabular}{|c|c|c|c|c|c|c|c|c|}
\hline Species & ID & Series & $\mathrm{U}-238(\mu \mathrm{g} / \mathrm{kg})$ & $\mathrm{U}-235(\mu \mathrm{g} / \mathrm{kg})$ & \begin{tabular}{|l|} 
U-235JU-238 \\
Mass Ratio
\end{tabular} & $\begin{array}{c}\text { U-238 Conc } \\
\text { (pCilg) }\end{array}$ & \# Individuals & \# Measurements \\
\hline & E5 & & 220.00 & & & $7.48 E-02$ & 1 & \\
\hline & 67 & & 110.00 & & & $3.74 E-02$ & 1 & \\
\hline & $\bar{\infty}$ & & 67.00 & & & $2.28 E-02$ & 1 & \\
\hline & 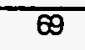 & & 220.00 & & & $7.48 \mathrm{E}-02$ & 1 & \\
\hline & 71 & & 69.00 & & & $2.35 E-02$ & 1 & \\
\hline & 74 & & 66.00 & & & $2.24 E-02$ & 1 & \\
\hline & 76 & & 66.00 & & & $2.24 E-02$ & 1 & \\
\hline & 78 & & 60.00 & & & $2.04 E-02$ & 1 & \\
\hline & 85 & & 150.00 & & & $5.10 E-02$ & 1 & \\
\hline & 87 & & 39.00 & & & $1.33 E-02$ & 1 & \\
\hline & 89 & & 40.00 & & & $1.36 E-02$ & 1 & \\
\hline & 91 & & 140.00 & & & $4.76 E-02$ & 1 & \\
\hline & 92 & & 75.00 & & & $2.55 E-02$ & 1 & \\
\hline & 94 & & 56.00 & & & $1.90 \mathrm{E}-02$ & 1 & \\
\hline & 96 & & 80.00 & & & $2.72 E-02$ & 1 & \\
\hline & 97 & & 79.00 & & & $2.69 E-02$ & 1 & \\
\hline & 98 & & 140.00 & & & $4.76 E-02$ & 1 & \\
\hline & 99 & & 130.00 & & & $4.42 E-02$ & 1 & \\
\hline & 100 & & 110.00 & & & $3.74 E-02$ & 1 & \\
\hline & 103 & & 110.00 & & & $3.74 E-02$ & 1 & \\
\hline & 3019 & & 94.00 & & & $3.20 E-02$ & 10 & \\
\hline & 3033 & & 40.00 & & & $1.36 E-02$ & 10 & \\
\hline & 3070 & & 110.00 & & & $3.74 E-02$ & 10 & \\
\hline & 3071 & & 310.00 & & & $1.05 E-01$ & 7 & \\
\hline & 3073 & & 71.00 & & & $2.41 E-02$ & 10 & \\
\hline & 3137 & & 270.00 & & & $9.18 E-02$ & 1 & \\
\hline & 3145 & & 99.00 & & & $3.37 E-02$ & 1 & \\
\hline & 3147 & & 42.00 & & & $1.43 E-02$ & 1 & \\
\hline & 3148 & & 58.00 & & & $1.97 E-02$ & 1 & \\
\hline & 3149 & & 43.00 & & & $1.46 E-02$ & 1 & \\
\hline & 3150 & & 18.00 & & & $6.12 E-03$ & 1 & \\
\hline & & & & & & & & \\
\hline \multicolumn{9}{|l|}{ Yeliow Perch } \\
\hline & 11 & $\bar{A}$ & 38.00 & & & $1.29 \mathrm{E}-02$ & 1 & \\
\hline & 11 & $\bar{B}$ & 630.00 & & & $2.14 E-01$ & 1 & \\
\hline & 35 & $\bar{A}$ & 83.00 & & & $2.82 E-02$ & 1 & \\
\hline & 35 & $\bar{B}$ & 98.00 & & & $3.33 E-02$ & 1 & \\
\hline & 42 & & 9200 & & & $3.13 \mathrm{E}-02$ & 1 & \\
\hline & 125 & liver & 200.00 & & & $6.80 \mathrm{E}-02$ & 2 & \\
\hline
\end{tabular}


Table C-2. (cont.)

\begin{tabular}{|c|c|c|c|c|c|c|c|c|}
\hline Species & ID & Series & $U-238(\mu \mathrm{g} / \mathrm{kg})$ & $U-235(\mu \mathrm{g} / \mathrm{kg}$ & $\begin{array}{l}\text { U-235/U-238 } \\
\text { Mass Ratio }\end{array}$ & $\begin{array}{l}\text { U-238 Conc } \\
\text { (pCi/g) }\end{array}$ & \# Individuals & \# Measurements \\
\hline & 126 & carcass & 810.00 & 5.50 & 0.0068 & $2.75 E-01$ & 2 & \\
\hline & 127 & fillets & 77.00 & & & $2.62 E-02$ & 2 & \\
\hline & 128 & kidney & 30.00 & & & $1.02 \mathrm{E}-02$ & 2 & \\
\hline & & & & & & & & \\
\hline & & & & & Ave & $4.68 E-02$ & & \\
\hline & & & & & Stdev & $5.17 E-02$ & & \\
\hline & & & & & Min & 2.55E-03 & & \\
\hline & & & & & $\overline{\operatorname{Max}}$ & $3.03 E-01$ & & \\
\hline & & & & & Count & 74 & & \\
\hline & & & & & $95 \% \mathrm{Cl}$ & $1.18 \mathrm{E}-02$ & & \\
\hline
\end{tabular}




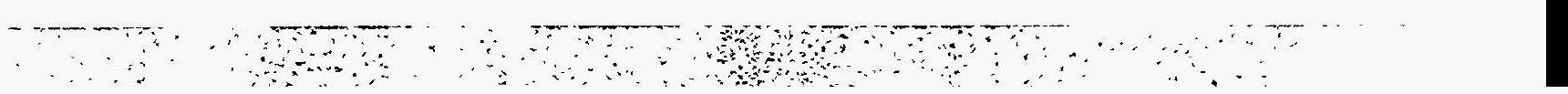




\section{Appendix D}

\section{Rate Equations for YPG Terrestrial Model}

D-1 
Table D-1. Rate equations used in YPG terrestrial model to estimate environmental fate of DU.

$\underline{\text { Vegetation Interior }}$

$\frac{d C_{2 i}}{d t}=C_{1} \frac{I_{2 i, 1}}{K_{d}}-C_{2 i}\left(I_{3,2 i}+I_{4,2 i}+I_{5,2 i}+I_{8,2 i}\right)$

Vegetation Surface

$\frac{d C_{2 s}}{d t}=C_{1} I_{2 s, 1}-C_{2 s}\left(I_{3,2 s}+I_{4,2 s}+I_{5,2 s}+I_{1,2 s}+I_{8,2 s}\right)$

Arthropods

$\frac{d C_{3}}{d t}=C_{1} I_{3,1}+\left(C_{2 i}+C_{2 s}\right)_{3,2}+C_{8} I_{3,8}-C_{3}\left(l_{14}+I_{4,3}+I_{6,3}+I_{8,3}\right)$

Small Herbivores

$\frac{d C_{4}}{d t}=C_{1} I_{1,4}+\left(C_{2 i}+C_{2 s}\right)_{4,2}+C_{3} I_{4,3}-C_{4}\left(I_{14}+I_{7,4}+I_{8,4}\right)$

Large Herbivores

$\frac{d C_{5}}{d t}=C_{1} I_{5,1}+\left(C_{2 i}+C_{2 s}\right)_{5,2}-C_{5}\left(I_{1,8}+I_{8,5}\right)$

Insectivores

$\frac{d C_{6}}{d t}=C_{1} l_{6,1}+C_{3} l_{6,3}-C_{6}\left(I_{1,6}+l_{7,6}+I_{8,6}\right)$

Predators

$\frac{d C_{7}}{d t}=C_{1} I_{7,1}+C_{4} I_{7,4}+C_{6} I_{7,6}-C_{7}\left(l_{1,7}+I_{8,7}\right)$

Litter

$$
\begin{aligned}
\frac{d C_{8}}{d t} & =\frac{\left(C_{2 i}+C_{2 s}\right)_{2} B_{8,2}+C_{3} B_{3} I_{8,3}+C_{4} B_{4} I_{8,4}+C_{5} B_{5} I_{8,5}+C_{6} B_{6} I_{2,6}+C_{7} B_{7} I_{2,7}}{B_{8}} \\
& \left.+\left(C_{2 i}+C_{2 s}\right)_{3,2}+I_{4,2}+I_{5,2}\right)+C_{3}\left(I_{4,3}^{\prime}+I_{6,3}\right)+C_{4} I_{7,4}+C_{6} I_{7,6}+C_{8} I_{3,8}^{\prime} \\
& -C_{8}\left(I_{1,8}+I_{3,8}\right)
\end{aligned}
$$


Table D-2. Parameter values and ranges used in the YPG terrestrial model uncertainty analysis.

\begin{tabular}{|c|c|c|c|c|c|}
\hline Parameter & $\begin{array}{c}\text { Nominal } \\
\text { Value }\end{array}$ & Range & Units & Explanation & Source \\
\hline$a_{4}$ & 0.15 & $0.09-0.24$ & $g / d$ & variable in equation predicting $\mathrm{FB}_{\mathrm{l}}$ & Nagy (1987) \\
\hline$a_{5}$ & 0.15 & $0.09-0.24$ & $g / d$ & variable in equation predicting $\mathrm{FB}_{\mathrm{l}}$ & Nagy (1987) \\
\hline$a_{6}$ & 0.65 & $0.49-0.86$ & g/d & variable in equation predicting $\mathrm{FB}_{\mathrm{j}}$ & Nagy (1987) \\
\hline a7 & 0.15 & $0.09-0.24$ & g/d & variable in equation predicting $\mathrm{FB}_{\mathbf{i}}$ & Nagy (1987) \\
\hline$a m_{4}$ & 8.57 & $\pm 50 \%$ & $\mathrm{~kJ} / \mathrm{d}$ & $\begin{array}{l}\text { variable in equation predicting metabolic rate and used } \\
\text { to estimate } I_{1, j}\end{array}$ & Calder (1984) \\
\hline$a_{5}$ & 8.4 & $\pm 50 \%$ & $\mathrm{~kJ} / \mathrm{d}$ & $\begin{array}{l}\text { variable in equation predicting metabolic rate and used } \\
\text { to estimate } I_{1, j}\end{array}$ & Calder (1984) \\
\hline$a m_{B}$ & 14.3 & $\pm 50 \%$ & $\mathrm{~kJ} / \mathrm{d}$ & $\begin{array}{l}\text { variable in equation predicting metabolic rate and used } \\
\text { to estimate } I_{1 \mathbf{j}}\end{array}$ & Calder (1984) \\
\hline$a m_{7}$ & 8.4 & $\pm 50 \%$ & $\mathrm{~kJ} / \mathrm{d}$ & $\begin{array}{l}\text { variable in equation predicting metabolic rate and used } \\
\text { to estimate } I_{1, j}\end{array}$ & Calder (1984) \\
\hline$a_{4}$ & 0.59 & $\pm 50 \%$ & $1 / y r$ & $\begin{array}{l}\text { variable in equation predicting annual survival rate and } \\
\text { used to estimate } I_{\mathbf{8} \mathbf{j}}\end{array}$ & Calder (1984) \\
\hline$a_{5}$ & 0.59 & $\pm 50 \%$ & $1 / \mathrm{yr}$ & $\begin{array}{l}\text { variable in equation predicting annual survival rate and } \\
\text { used to estimate }\left.\right|_{8 \mathbf{j}}\end{array}$ & Calder (1984) \\
\hline$a_{6}$ & 1.38 & $\pm 50 \%$ & $1 / y r$ & $\begin{array}{l}\text { variable in equation predicting annual survival rate and } \\
\text { used to estimate } I_{8, j}\end{array}$ & Calder (1984) \\
\hline as7 & 0.59 & $\pm 50 \%$ & $1 /$ hr & $\begin{array}{l}\text { variable in equation predicting annual survival rate and } \\
\text { used to estimate }\left.\right|_{\mathbf{8} \mathbf{j}}\end{array}$ & Calder (1984) \\
\hline $\mathbf{B}_{2}$ & 0.7 & $0.1-2$ & $\mathrm{~kg} / \mathrm{m}^{2}$ & dry mass in compartment 2: vegetation & Begon et al. (1990:652) \\
\hline $\mathbf{b}_{4}$ & 0.79 & $0.73-0.84$ & $1 / d$ & variable in equation predicting $\mathrm{FB}_{\mathrm{i}}$ & Nagy (1987) \\
\hline$B_{4}$ & 1.13E-04 & 8.82E-05-1.38E-04 & $\mathrm{kg} / \mathrm{m}^{2}$ & dry mass in compartment 4: small herbivores & Chew and Chew (1970) \\
\hline $\mathbf{b}_{5}$ & -0.79 & $0.73-0.84$ & $1 / d$ & variable in equation predicting $\mathrm{FB}_{\mathrm{I}}$ & Nagy (1987) \\
\hline $\mathbf{b}_{6}$ & 0.65 & $-0.60-0.70$ & $1 / d$ & variable in equation predicting $\mathrm{FB}_{\mathfrak{i}}$ & Nagy (1987) \\
\hline$b_{7}$ & 0.79 & $0.73-0.84$ & $1 / d$ & variable in equation predicting $\mathrm{FB}_{\mathfrak{l}}$ & Nagy (1987) \\
\hline BECV & 0.02 & $0.01-0.1$ & unitless & efficiency of biomass conversion by predators & \\
\hline$B E_{H V}$ & 0.02 & $0.01-0.1$ & unitless & efficiency of biomass conversion by herbivores & \\
\hline$B E_{N}$ & 0.02 & $0.01-0.1$ & unitless & efficiency of biomass conversion by insectivores & \\
\hline $\mathrm{bm}_{4}$ & 0.54 & $\pm 30 \%$ & unitless & $\begin{array}{l}\text { variable in equation predicting metabolic rate and used } \\
\text { to estimate I iJ }\end{array}$ & Calder (1984) \\
\hline $\mathbf{b m}_{5}$ & 0.66 & $\pm 30 \%$ & unitless & $\begin{array}{l}\text { variable in equation predicting metabolic rate and used } \\
\text { to estimate }\left.\right|_{1, j}\end{array}$ & Calder (1984) \\
\hline $\mathbf{b m}_{6}$ & 0.43 & $\pm 30 \%$ & unitiess & $\begin{array}{l}\text { variable in equation predicting metabolic rate and used } \\
\text { to estimate } l_{1, j}\end{array}$ & Calder (1984) \\
\hline $\mathbf{b m}_{7}$ & 0.66 & $\pm 30 \%$ & unitless & $\begin{array}{l}\text { variable in equation predicting metabolic rate and used } \\
\text { to estimate } I_{1, j}\end{array}$ & Calder (1984) \\
\hline$b_{4}$ & 0.56 & $\pm 30 \%$ & unitless & $\begin{array}{l}\text { variable in equation predicting annual survival rate and } \\
\text { used to estimate } I_{8 \mathrm{~J}}\end{array}$ & Calder (1984) \\
\hline bs5 $_{5}$ & 0.56 & $\pm 30 \%$ & unitless & $\begin{array}{l}\text { variable in equation predicting annual survival rate and } \\
\text { used to estimate } I_{8 j}\end{array}$ & Calder (1984) \\
\hline $\mathrm{bs}_{6}$ & 0.36 & $\pm 30 \%$ & unitless & $\begin{array}{l}\text { variable in equation predicting annual survival rate and } \\
\text { used to estimate }\left.\right|_{8 \mathrm{~J}}\end{array}$ & Calder (1984) \\
\hline
\end{tabular}


Table D-2. (cont.)

\begin{tabular}{|c|c|c|c|c|c|}
\hline Parameter & $\begin{array}{l}\text { Nominal } \\
\text { Value }\end{array}$ & Range & Units & Explanation & Source \\
\hline$b_{7}$ & 0.56 & $\pm 30 \%$ & unitless & $\begin{array}{l}\text { variable in equation predicting annual survival rate and } \\
\text { used to estimate } 1_{8 \mathrm{~J}}\end{array}$ & Calder (1984) \\
\hline$c_{1}$ & 1 & - & $\mathrm{mg}$ DU/kg & assumes initial soil concentration & - \\
\hline $\mathrm{C}_{2}$ & 0 & - & mg DU/kg & DU plant concentration $\left(\mathrm{C}_{2 !}+\mathrm{C}_{2 \mathrm{~S}}\right)$ & - \\
\hline $\mathrm{C}_{2 !}$ & 0 & - & $\mathrm{mg} \mathrm{DU} / \mathrm{kg}$ & DU in vegetation & - \\
\hline $\mathrm{C}_{2 \mathrm{~S}}$ & 0 & - & $\mathrm{mg} \mathrm{DU} / \mathrm{kg}$ & DU on vegetation & - \\
\hline $\mathrm{C}_{3}$ & 0 & 一 & $\mathrm{mg}$ DU/kg & DU in invertebrates & - \\
\hline $\mathrm{C}_{4}$ & 0 & - & $\mathrm{mg} \mathrm{DU} / \mathrm{kg}$ & DU in small herbivores & - \\
\hline$C_{5}$ & 0 & - & $\mathrm{mg} D U \mathrm{~kg}$ & DU in large herbivores & - \\
\hline $\mathrm{C}_{6}$ & 0 & - & $\mathrm{mg} \mathrm{DU} / \mathrm{kg}$ & DU in insectivores & - \\
\hline$C_{7}$ & 0 & - & $\mathrm{mg} \mathrm{DU/kg}$ & DU in predators & - \\
\hline $\mathrm{C}_{8}$ & 0 & - & $\mathrm{mg}$ DU/kg & DU in litter & - \\
\hline $\mathrm{DM}_{4}$ & 0.3 & $0.2-0.6$ & unittess & dry matter content & \\
\hline $\mathrm{DM}_{5}$ & 0.3 & $0.2-0.6$ & unitiess & dry matter content & \\
\hline $\mathrm{DM}_{6}$ & 0.3 & $0.2-0.6$ & unitless & dry matter content & \\
\hline $\mathrm{DM}_{7}$ & 0.3 & $0.2-0.6$ & unittess & dry matter content & \\
\hline$E_{3,1}$ & 0.01 & $0.0035-0.02$ & unitless & DU assimilation coefficient & Wrenn et al. (1985) \\
\hline$E_{3,2}$ & 0.01 & $0.0035-0.02$ & unitless & DU assimilation coefficient & Wrenn et al. (1985) \\
\hline$E_{3,8}$ & 0.01 & $0.0035-0.02$ & unitless & DU assimilation coefficient & Wrenn et al. (1985) \\
\hline$E_{4,1}$ & 0.01 & $0.0035-0.02$ & unitless & DU assimilation coefficient & Wrenn et al. (1985) \\
\hline$E_{4,2}$ & 0.01 & $0.0035-0.02$ & unitless & DU assimilation coefficient & Wrenn et al. (1985) \\
\hline$E_{4,3}$ & 0.01 & $0.0035-0.02$ & unittess & DU assimilation coefficient & Wrenn et al. (1985) \\
\hline$E_{5,1}$ & 0.01 & $0.0035-0.02$ & unitiess & DU assimilation coefficient & Wrenn et al. (1985) \\
\hline$E_{5,2}$ & 0.01 & $0.0035-0.02$ & unitiess & DU assimilation coefficient & Wrenn et al. (1985) \\
\hline$E_{6,1}$ & 0.01 & $0.0035-0.02$ & unitless & DU assimilation coefficient & Wrenn et al. (1985) \\
\hline$E_{6,3}$ & 0.01 & $0.0035-0.02$ & unitless & DU assimilation coefficient & Wrenn et al. (1985) \\
\hline$E_{7.1}$ & 0.01 & $0.0035-0.02$ & unitiess & DU assimilation coefficient & Wrenn et al. (1985) \\
\hline$E_{7,3}$ & 0.01 & $0.0035-0.02$ & unitless & DU assimilation coefficient & Wrenn et al. (1985) \\
\hline$E_{7.4}$ & 0.01 & $0.0035-0.02$ & unitless & DU assimilation coefficient & Wrenn et al. (1985) \\
\hline$E_{7,4}$ & 0.01 & $0.0035-0.02$ & unitless & DU assimilation coefficient & Wrenn et al. (1985) \\
\hline$E_{7,6}$ & 0.01 & $0.0035-0.02$ & unitless & DU assimilation coefficient & Wrenn et al. (1985) \\
\hline ER & 0.5 & $0.2-0.8$ & unitless & erosion loss & \\
\hline$f_{30}$ & 0.76 & \pm 0.06 & ng & variable in equation for predicting $\left.\right|_{1,3}$ & van Straalen et al. (1987) \\
\hline$f_{3 i}$ & 2.3 & \pm 0.04 & ng & variable in equation for predicting $\left.\right|_{1,3}$ & van Straalen et al. (1987) \\
\hline$f \mathrm{~b}_{3}$ & 0.07 & $0.02-0.3$ & unitless & dry matter intake as a fraction of $B_{3}$ & \\
\hline $\mathrm{FD}_{3,8}$ & 0.73 & $0.37-0.93$ & unitless & $\begin{array}{l}\text { fraction of diet for compartment } 3 \text { coming from } \\
\text { compartment } 8 . \mathrm{FD}_{3,2}=1-\mathrm{FD}_{3,8} \text { so that } \overline{\mathrm{A}} F D_{3 \mathrm{~J}}=1\end{array}$ & \\
\hline $\mathrm{FD}_{4,2}$ & 0.9 & $0.8-0.95$ & unitless & $\begin{array}{l}\text { fraction of diet for compartment } 4 \text { coming from } \\
\text { compartment } 2 . \mathrm{FD}_{4,3}=1-\mathrm{FD}_{4,2} \text { so that } \overline{\mathrm{A}} \mathrm{FD}_{4, \mathrm{j}}=1\end{array}$ & \\
\hline $\mathrm{FD}_{7,3}$ & 0.5 & $0-1$ & unitless & $\begin{array}{l}\text { fraction of diet for compartment } 7 \text { coming from } \\
\text { compartment } 3 \text {. FD } F D_{7.4}=\left(1-F D_{7.3}\right)^{*} p s h v \text { and } F D_{7.6}=(1 \\
\left.-F D_{7,3}\right)^{*}\left(1-\text { pshiv so that } \bar{A} F D_{7, J}=1\right.\end{array}$ & \\
\hline $\mathrm{FHV}_{3}$ & 0.55 & $0.3-0.8$ & unitless & fraction of herbivore biomass as 3 & \\
\hline $\mathrm{FHV}_{5}$ & 0.05 & $0.01-0.1$ & unitless & fraction of herbivore biomass as 5 & \\
\hline$f r$ & 0.08 & $0.06-0.10$ & unitless & estimates mass of litter from $B_{2}$ & $\begin{array}{l}\text { Klemedson and Barth } \\
\text { (1974) }\end{array}$ \\
\hline
\end{tabular}


Table D-2. (cont.)

\begin{tabular}{|c|c|c|c|c|c|}
\hline Parameter & $\begin{array}{c}\text { Nominal } \\
\text { Value }\end{array}$ & Range & Units & Explanation & Source \\
\hline$f s_{3}$ & 0.04 & $0-0.2$ & unitless & soil intake as fraction of daily intake & $\begin{array}{l}\text { Garten (1980), Zach and } \\
\text { Mayo (1984) }\end{array}$ \\
\hline$f s_{4}$ & 0.04 & $0-0.2$ & unitless & soil intake as fraction of daily intake & $\begin{array}{l}\text { Garten (1980), Zach and } \\
\text { Mayo (1984) }\end{array}$ \\
\hline$f \mathrm{~s}_{5}$ & 0.04 & $0-0.2$ & unitless & soil intake as fraction of daily intake & $\begin{array}{l}\text { Garten (1980), Zach and } \\
\text { Mayo (1984) }\end{array}$ \\
\hline$f s_{6}$ & 0.04 & $0-0.2$ & unittess & soil intake as fraction of daily intake & $\begin{array}{l}\text { Garten (1980), Zach and } \\
\text { Mayo (1984) }\end{array}$ \\
\hline$f_{7}$ & 0.04 & $0-0.2$ & unitiess & soil intake as fraction of daily intake & $\begin{array}{l}\text { Garten (1980), Zach and } \\
\text { Mayo (1984) }\end{array}$ \\
\hline $\mathrm{FU}_{4}$ & 2.0 & $0.3-3.0$ & $1 / \mathrm{kJ}$ & scales metabolic rate $(\mathrm{kJ} / \mathrm{d})$ to elimination rate $(1 / \mathrm{d})$ & \\
\hline $\mathrm{Fu}_{5}$ & 2.0 & $0.3-3.0$ & $1 / \mathrm{kJ}$ & scales metabolic rate $(\mathrm{kJ} / \mathrm{d})$ to elimination rate $(1 / \mathrm{d})$ & \\
\hline $\mathrm{FU}_{6}$ & 2.0 & $0.3-3.0$ & $1 / \mathrm{kJ}$ & scales metabolic rate $(\mathrm{kJ} / \mathrm{d})$ to elimination rate $(1 / \mathrm{d})$ & \\
\hline $\mathrm{FU}_{7}$ & 2.0 & $0.3-3.0$ & $1 / \mathrm{kJ}$ & scales metabolic rate $(\mathrm{kJ} / \mathrm{d})$ to elimination rate $(1 / \mathrm{d})$ & \\
\hline kd & 1080 & $180-3 E 05$ & unittess & partitions bound DU/available DU in soil & $\begin{array}{l}\text { Simon (1985) and Sheppard } \\
\text { and Evender (1988) }\end{array}$ \\
\hline$k_{r}$ & 0.00086 & $\pm 50 \%$ & $1 / d$ & rainsplash rate & $\begin{array}{l}\text { Whicker and Kirchner } \\
\text { (1987) see Dreicer et al. } \\
1984\end{array}$ \\
\hline$k_{w}$ & 0.0495 & $\pm 50 \%$ & $1 / d$ & weathering rate & $\begin{array}{l}\text { Whicker and Kirchner } \\
\text { (1987) see Ho79 }\end{array}$ \\
\hline $1,1,8$ & 0.0015 & $0.00099-0.0023$ & $1 / d$ & lifter to soil transfer & Santos et al. (1984) \\
\hline$I_{2,1}$ & 0.36 & $\pm 50 \%$ & $1 / d$ & plant uptake from soil & Simon (1985) \\
\hline 18,2 & 0.0012 & $0.00019-0.0023$ & $1 / d$ & litter fall & Strojan et al. (1979) \\
\hline PS & 1460 & $\pm 50 \%$ & $\mathrm{~kg} / \mathrm{m}^{3}$ & soil bulk density & $\begin{array}{l}\text { Whicker and Kirchner } \\
\text { (1987) }\end{array}$ \\
\hline pshv & 0.5 & $0.25-0.75$ & unitless & partitions intake by predators & \\
\hline $\mathbf{R F}$ & 0.0001 & $10^{-10}-10^{-2}$ & $1 / \mathrm{m}$ & resuspension factor & $\begin{array}{l}\text { Whicker and Kirchner } \\
\text { (1987) see Anspugh et al. } \\
1975\end{array}$ \\
\hline v & 173 & $2.6-4900$ & $\mathrm{~m} / \mathrm{d}$ & deposition velocity & $\begin{array}{l}\text { Whicher and Kirchner } \\
\text { (1987) see Whicher \& } \\
\text { Schulz 1982, mi83 }\end{array}$ \\
\hline$W_{3}$ & 0.05 & $\pm 50 \%$ & individual & live body mass & Remmert (1981) \\
\hline$W_{4}$ & 65 & $58-73$ & individual & live body mass & Chew and Chew (1970) \\
\hline$W_{5}$ & 40,000 & $10,000-60,000$ & $\underset{\text { individual }}{g^{\prime}}$ & live body mass & Nagy (1987) \\
\hline$W_{B}$ & 50 & $25-300$ & individual & live body mass & Nagy (1987) \\
\hline$W_{7}$ & 5,000 & $1,000-10,000$ & individual & live body mass & Nagy (1987) \\
\hline $\mathbf{Z}_{\mathbf{s}}$ & 0.001 & $0.0005-0.0015$ & $\mathbf{m}$ & labile soil depth & Whicker and Kirchner 1987 \\
\hline
\end{tabular}




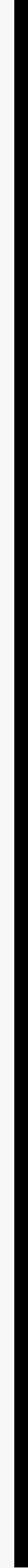


Appendix E

Data from YPG Field Samples

E-1 
Table E-1. Data for Soil Compartment $\left(C_{1}\right)$, YPG. Data for all samples listed, and descriptive statistics are shown. Negative values indicate samples less than the detection limit, and the detection limit was the absolute value of the reported negative number. NA indicates a value is inappropriate, and nd indicates that no data were reported.

\begin{tabular}{|c|c|c|c|c|c|c|c|}
\hline ID & Series & ${ }^{238} \mathrm{U}$ (mg/kg) & ${ }^{235} \mathrm{U}(\mathrm{mg} / \mathrm{kg})$ & ${ }^{235} \mathrm{U} /^{238} \mathrm{U}$ & Net Wet (g) & Net Oven (g) & $\begin{array}{l}\text { U-238 Conc } \\
\text { (pCi/g) }\end{array}$ \\
\hline 244 & & $2.20 \mathrm{E}+05$ & 600 & 0.0027 & 252.15 & 249.05 & $8.14 E+01$ \\
\hline 245 & & $4.30 \mathrm{E}+04$ & 220 & 0.0051 & 224.49 & 221.47 & $1.59 \mathrm{E}+01$ \\
\hline 248 & & $1.10 E+05$ & 420 & 0.0038 & 242.16 & 239.69 & $4.07 \mathrm{E}+01$ \\
\hline 249 & & $1.40 E+05$ & 600 & 0.0043 & 186.63 & 183.97 & $5.18 \mathrm{E}+01$ \\
\hline 252 & & $2.10 E+04$ & 52 & 0.0025 & 250.27 & 246.98 & $7.77 \mathrm{E}+00$ \\
\hline 253 & & $4.30 E+04$ & 220 & 0.0051 & 22284 & 220.18 & $1.59 \mathrm{E}+01$ \\
\hline 258 & & $6.00 E+05$ & 2600 & 0.0043 & 236.49 & 233.55 & $2.22 E+02$ \\
\hline 259 & & $8.20 \mathrm{E}+05$ & 2800 & 0.0034 & 317.98 & 313.56 & $3.03 E+02$ \\
\hline 265 & & $5.50 E+04$ & 260 & 0.0047 & 177.51 & 175.79 & $2.04 E+01$ \\
\hline 266 & & $210 E+04$ & 150 & 0.0071 & 259.13 & 256.37 & $7 . \overline{77 E+00}$ \\
\hline 268 & & $1.20 \mathrm{E}+06$ & 5600 & 0.0047 & 150.11 & 148.36 & $4.44 E+02$ \\
\hline 270 & & $1.40 E+06$ & 4200 & 0.0030 & 239.43 & 236.49 & $5.18 E+02$ \\
\hline 274 & & $2.40 \mathrm{E}+04$ & 120 & 0.0050 & 239.06 & 236.85 & $8.88 \mathrm{E}+00$ \\
\hline 280 & & $4.10 E+04$ & 270 & 0.0066 & 311.95 & 307.02 & $1.52 E+01$ \\
\hline 281 & & $2.70 E+03$ & 3.5 & 0.0013 & 30239 & 297.94 & $9.99 \mathrm{E}-01$ \\
\hline 284 & & $2.10 E+02$ & nd & nd & 12.49 & 11.70 & $7.77 E-02$ \\
\hline 286 & & $2.50 E+04$ & 37.5 & 0.0015 & 229.33 & 225.33 & $9.25 E+00$ \\
\hline 288 & & $1.30 E+04$ & 47 & 0.0036 & 318.12 & 312.38 & $4.81 E++0$ \\
\hline 294 & & $2.60 \mathrm{E}+04$ & 94 & 0.0036 & 207.38 & 200.51 & $9.62 E+00$ \\
\hline 295 & & $3.80 E+04$ & 106 & 0.0028 & 205.42 & 203.02 & $1.41 E+01$ \\
\hline 296 & & $-2.50 E+00$ & nd & nd & 205.42 & 203.20 & $-9.25 E-04$ \\
\hline 254 & & $1.00 E+05$ & 440 & 0.0044 & nd & nd & $3.70 \mathrm{E}+01$ \\
\hline & & & & & & $\begin{array}{c}\text { No. Samples } \\
\text { > DL }\end{array}$ & 22 \\
\hline & & & & & & Ave & $8.3 E+01$ \\
\hline & & & & & & Median & $1.6 \mathrm{E}+01$ \\
\hline & & & & & & Std. Dev. & $1.5 \mathrm{E}+02$ \\
\hline & & & & & & $95 \% \mathrm{CI}$ & $6.2 E+01$ \\
\hline & & & & & & Upper 95\% & $1.5 E+02$ \\
\hline & & & & & & Lower 95\% & $2.1 \mathrm{E}+01$ \\
\hline
\end{tabular}


Table E-2. Data for Litter Compartment $\left(\mathrm{C}_{8}\right)$, YPG. Data for all samples listed, and descriptive statistics are shown. Only averages of replicates used in statistical calulations. Negative values indicate samples less than the detection limit, and the detection limit was the absolute value of the reported negative number. NA indicates a value is inappropriate, and nd indicates that no data were reported.

\begin{tabular}{|c|c|c|c|c|c|c|c|c|}
\hline ID & Series & ${ }^{238} \mathrm{U}(\mathrm{mg} / \mathrm{kg})$ & ${ }^{235} \mathrm{U}(\mathrm{mg} / \mathrm{kg})$ & ${ }^{235} \mathrm{U} /{ }^{238} \mathrm{U}$ & Net Wet (g) & Net Oven (g) & Net Ash (g) & $\begin{array}{l}\text { Total U } \\
\text { (pcilg) Fresh } \\
\text { Wt. }\end{array}$ \\
\hline 243 & $\bar{A}$ & - 5.10E+05 & 380 & 0.0007 & 8.74 & 7.94 & 284 & $6.13 E+01$ \\
\hline 243 & B & $3.10 \mathrm{E}+05$ & 600 & 0.0019 & 8.74 & 7.94 & 284 & $3.73 E+01$ \\
\hline 243 & Ave. & $4.10 E+05$ & NA & NA & $\mathrm{NA}$ & NA & $\mathrm{NA}$ & $4.93 E+01$ \\
\hline 246 & & $2.70 E+05$ & 1300 & 0.0048 & 10.00 & 9.11 & 2.87 & $2.87 \mathrm{E}+01$ \\
\hline 247 & $\mathrm{~A}$ & $3.90 E+05$ & 1800 & 0.0046 & 6.84 & 6.30 & 290 & $6.12 \mathrm{E}+01$ \\
\hline 247 & B & $3.60 \mathrm{E}+05$ & 460 & 0.0013 & 6.84 & 6.30 & 2.90 & $5.65 \mathrm{E}+01$ \\
\hline 247 & Ave. & 3.75E+05 & NA & NA & NA & NA & NA & $5.88 \mathrm{E}+01$ \\
\hline 250 & & $9.60 \mathrm{E}+04$ & 330 & 0.0034 & 9.19 & 8.31 & 2.09 & $8.08 E+00$ \\
\hline 251 & $\bar{A}$ & $1.90 E+05$ & 560 & 0.0029 & 11.74 & 10.91 & 3.41 & $2.04 E+01$ \\
\hline 251 & $\bar{B}$ & 1.70E+05 & 330 & 0.0019 & 11.74 & 10.91 & 3.41 & $1.83 \mathrm{E}+01$ \\
\hline 251 & Ave. & $1.80 E+05$ & NA & $\mathrm{NA}$ & $\mathrm{NA}$ & $\mathrm{NA}$ & $\overline{N A}$ & $4.40 \mathrm{E}+01$ \\
\hline 256 & & $1.00 \mathrm{E}+06$ & 2400 & 0.0024 & 2.34 & 212 & 0.33 & 5.22E+01 \\
\hline 257 & & $7.90 \mathrm{E}+05$ & 2300 & 0.0029 & 10.58 & 9.67 & 3.84 & $1.06 \mathrm{E}+02$ \\
\hline 261 & & $3.80 E+04$ & 200 & 0.0053 & 1.90 & 1.72 & 0.32 & $2.37 \mathrm{E}+00$ \\
\hline 263 & & $1.40 \mathrm{E}+05$ & 434 & 0.0031 & 5.75 & 528 & 1.36 & $1.23 E+01$ \\
\hline 267 & $\bar{A}$ & $1.10 \mathrm{E}+06$ & 3500 & 0.0032 & 6.10 & 5.70 & 3.03 & $2.02 E+02$ \\
\hline 267 & $\mathrm{~B}$ & $1.30 \mathrm{E}+06$ & 1700 & 0.0013 & 6.10 & 5.70 & 3.03 & $2.39 E+02$ \\
\hline 267 & Ave. & $1.20 \mathrm{E}+06$ & NA & NA & NA & NA & NA & $2.21 E+02$ \\
\hline 275 & & $6.20 \mathrm{E}+04$ & 460 & 0.0074 & 6.62 & 6.02 & 1.09 & $3.78 \mathrm{E}+00$ \\
\hline 276 & & $7.70 E+03$ & 14 & 0.0018 & 10.48 & 9.54 & 233 & $6.33 E-01$ \\
\hline 278 & $\bar{A}$ & $4.80 E+04$ & 160 & 0.0033 & 15.74 & 14.70 & 5.85 & $6.60 E+00$ \\
\hline 278 & $\mathrm{~B}$ & $5.20 \mathrm{E}+04$ & 230 & 0.0044 & 15.74 & 14.70 & 5.85 & $7.15 E+00$ \\
\hline 278 & Ave. & $5.00 \mathrm{E}+04$ & NA & NA & NA & $\mathrm{NA}$ & NA & $6.88 \mathrm{E}+00$ \\
\hline 285 & & $1.10 E+03$ & 21 & 0.0019 & 7.05 & 6.46 & 234 & 1.35E-01 \\
\hline 287 & $\bar{A}$ & $2.30 \mathrm{E}+04$ & 56 & 0.0024 & 9.35 & 8.56 & 1.84 & 1.67E+00 \\
\hline 287 & B & $2.30 \mathrm{E}+04$ & 101 & 0.0044 & 9.35 & 8.56 & 1.84 & $1.67 \mathrm{E}+00$ \\
\hline 287 & Ave. & $2.30 \mathrm{E}+04$ & NA & NA & NA & NA & NA & 1.67E+00 \\
\hline 291 & & $9.20 \mathrm{E}+04$ & 195 & 0.0021 & 9.78 & 9.03 & 3.66 & $1.27 \mathrm{E}+01$ \\
\hline 293 & & $6.20 \mathrm{E}+04$ & 230 & 0.0037 & 10.92 & 10.08 & 230 & $4.83 E+00$ \\
\hline 320 & & $1.20 \mathrm{E}+06$ & 4200 & 0.0035 & 10.49 & 9.47 & 1.35 & $5.71 E+01$ \\
\hline \multirow[t]{8}{*}{326} & & $3.20 E+05$ & 3200 & 0.0100 & 238 & 1.19 & 0.62 & $3.08 \mathrm{E}+01$ \\
\hline & & & & & & & $\begin{array}{c}\text { No. Samples } \\
>\mathrm{DL}\end{array}$ & 18 \\
\hline & & & & & & & Ave & 3.7E+01 \\
\hline & & & & & & & Median & $1.3 E+01$ \\
\hline & & & & & & & Std. Dev. & $5.3 E+01$ \\
\hline & & & & & & & $95 \% \mathrm{Cl}$ & $2.4 \mathrm{E}+01$ \\
\hline & & & & & & & Upper 95\% & $6.2 \mathrm{E}+01$ \\
\hline & & & & & & & Lower 95\% & $1.3 \mathrm{E}+01$ \\
\hline
\end{tabular}


Table E-3. Data for Vegetation Compartment $\left(\mathbf{C}_{2}\right)$, YPG. Data for all samples listed, and descriptive statistics are shown. Negative values indicate samples less than the detection limit, and the detection limit was the absolute value of the reported negative number. NA indicates a value is inappropriate, and nd indicates that no data were reported

Vegetation

\begin{tabular}{|c|c|c|c|c|c|c|c|c|}
\hline ID & Series & ${ }^{238} \mathrm{U}(\mathrm{mg} / \mathrm{kg})$ & ${ }^{235} \mathrm{U}(\mathrm{mg} / \mathrm{kg})$ & ${ }^{235} \mathrm{U} I^{238} \mathrm{U}$ & Net Wet (g) & Net Oven (g) & Net Ash (g) & $\begin{array}{c}\text { Total U } \\
\text { (pCi/g) Fresh } \\
\text { Wt. }\end{array}$ \\
\hline 241 & & 540000.00 & 1300 & 0.0024 & 12.35 & 6.39 & 0.66 & $1.07 E+01$ \\
\hline 242 & & 110000.00 & 380 & 0.0035 & 24.94 & 13.37 & 1.08 & $1.76 E+\infty 0$ \\
\hline 254 & & 14000.00 & 41 & 0.0029 & 1251 & 4.97 & 0.88 & $3.64 E-01$ \\
\hline 255 & & 0.00 & nd & nd & 19.43 & 10.04 & 1.07 & $0.00 E+\infty 0$ \\
\hline 260 & & 780000.00 & 910 & 0.0012 & 10.48 & 5.13 & 0.59 & $1.62 E+01$ \\
\hline 264 & & 100000.00 & 250 & 0.0025 & 823 & 4.75 & 0.86 & $3.87 E+\infty 0$ \\
\hline 271 & & 240000.00 & 1200 & 0.0050 & 11.23 & 5.38 & 0.67 & $5.30 E+\infty 0$ \\
\hline 272 & & 20000.00 & -9 & nd & 1.59 & -54.23 & 0.13 & 6.05E-01 \\
\hline 273 & & 65000.00 & 170 & 0.0026 & 18.37 & -56.34 & 1.45 & $1.90 E+00$ \\
\hline 277 & & 4900.00 & 24 & 0.0049 & 1280 & 256 & 0.48 & $6.80 \mathrm{E}-02$ \\
\hline 279 & & 31000.00 & 98 & 0.0032 & 10.64 & 2.13 & 0.85 & $9.16 E-01$ \\
\hline 282 & & 10000.00 & 34 & 0.0034 & 11.56 & 231 & 0.86 & $2.75 E-01$ \\
\hline 283 & & 3400.00 & -1.75 & nd & 18.85 & 3.77 & 1.02 & $6.81 E-02$ \\
\hline 305 & & 4800.00 & -1.75 & $\mathrm{nd}$ & 14.94 & 299 & 0.82 & 9.75E-02 \\
\hline 306 & A & 110000.00 & 350 & 0.0032 & 15.92 & 12.74 & 7.79 & $1.99 \mathrm{E}+01$ \\
\hline 306 & $\mathrm{~B}$ & 14000.00 & 66 & 0.0047 & 15.92 & 1274 & 7.76 & $2.52 \mathrm{E}+00$ \\
\hline 306 & Ave. & 62000.00 & $\mathrm{NA}$ & NA & NA & NA & NA & $1.12 \mathrm{E}+01$ \\
\hline 307 & & 66000.00 & 183 & 0.0028 & 7.89 & 1.58 & 0.89 & $2.75 E+00$ \\
\hline 308 & & 58000.00 & 180 & 0.0031 & 8.55 & 1.71 & 1.13 & $2.84 E+00$ \\
\hline 309 & & 12000.00 & 88 & 0.0073 & 252 & 0.55 & 0.14 & $2.47 E-01$ \\
\hline 310 & & 220000.00 & 380 & 0.0017 & 13.54 & 271 & 0.77 & $4.63 E+00$ \\
\hline 311 & & 1000.00 & nd & nd & 0.54 & 0.48 & 0.02 & $1.37 E-02$ \\
\hline 312 & & 130000.00 & 420 & 0.0032 & 0.70 & 0.55 & 0.05 & $3.44 E+00$ \\
\hline 313 & & 66000.00 & 740 & 0.0112 & 0.73 & 0.51 & 0.03 & $1.00 \mathrm{E}+00$ \\
\hline 314 & & 170000.00 & nd & nd & 0.45 & 0.35 & 0.04 & $5.59 \mathrm{E}+00$ \\
\hline 315 & & 7800.00 & nd & nd & 0.46 & 0.35 & 0.01 & $6.27 E-02$ \\
\hline 316 & & 7400.00 & nd & nd & 1.79 & 0.46 & 0.05 & $7.65 E-02$ \\
\hline 317 & & 220000.00 & 1200 & 0.0055 & 5.63 & 1.13 & 0.60 & $8.67 E+00$ \\
\hline 318 & & 1600000.00 & 2000 & $\overline{0.0013}$ & 11.11 & 222 & 127 & $6.77 E+01$ \\
\hline 319 & & 60000.00 & 330 & 0.0055 & 0.18 & 0.10 & 0.02 & $2.47 E+\infty 0$ \\
\hline 321 & & 30000.00 & 85 & 0.0028 & 9.74 & 205 & 1.71 & $1.95 E+\infty 0$ \\
\hline 322 & A & 1400000.00 & 5500 & 0.0039 & 14.17 & 283 & 2.52 & $9.21 \mathrm{E}+01$ \\
\hline 322 & B & $\$ 700000.00$ & 5300 & 0.0031 & 14.17 & 283 & 252 & $1.12 E+02$ \\
\hline 322 & Ave. & 1550000.00 & $\mathrm{NA}$ & $\mathrm{NA}$ & NA & NA & $\mathrm{NA}$ & $1.02 E+02$ \\
\hline 323 & & 50000.00 & 200 & 0.0040 & 3.90 & 0.78 & 0.29 & $1.38 E+00$ \\
\hline 324 & & 86000.00 & 520 & 0.0060 & 14.35 & 2.87 & 0.90 & $2.00 \mathrm{E}+\infty 0$ \\
\hline 325 & & 36000.00 & 69 & 0.0019 & 3.85 & 3.08 & 1.88 & $6.50 E+00$ \\
\hline & & & & & & & $\begin{array}{c}\text { No. Samples > } \\
D L\end{array}$ & 33 \\
\hline & & & & & & & Ave & $8.1 E+00$ \\
\hline & & & & & & & Median & $1.9 E+00$ \\
\hline & & & & & & & Std. Dev. & $2.1 E+01$ \\
\hline & & & & & & & $95 \% \mathrm{Cl}$ & $7.0 E+00$ \\
\hline & & & & & & & Upper 95\% & $1.5 \mathrm{E}+01$ \\
\hline & & & & & & & Lower 95\% & $1.0 \mathrm{E}+00$ \\
\hline
\end{tabular}


Table E-4. Data for Invetebrates Compartment $\left(\mathrm{C}_{3}\right)$, YPG. Data for all samples listed, and descriptive statistics are shown. Negative values indicate samples less than the detection limit, and the detection limit was the absolute value of the reported negative number. NA indicates a value is inappropriate, and nd that no data were reported.

Invertebrates

\begin{tabular}{|c|c|c|c|c|c|c|c|c|}
\hline ID & Series & ${ }^{238} \mathrm{U}(\mathrm{mg} / \mathrm{kg})$ & ${ }^{235} \mathrm{U}(\mathrm{mg} / \mathrm{kg})$ & ${ }^{235} \mathrm{U} / \mathrm{I}^{238} \mathrm{U}$ & Net Wet (g) & Net Oven (g) & Net Ash (g) & $\begin{array}{l}\text { Total U (pclg) } \\
\text { Fresh Wt. }\end{array}$ \\
\hline 1 & & $2.10 E+03$ & 3 & 0.0014 & 0.46 & 0.58 & 0.00 & $9.80 \mathrm{E}-01$ \\
\hline 2 & & $2.10 E+04$ & 100 & 0.0048 & 0.37 & 0.17 & 0.00 & $3.57 \mathrm{E}+00$ \\
\hline 3 & & $1.10 \mathrm{E}+03$ & 2.9 & 0.0026 & 1.63 & 0.74 & 0.00 & $1.85 \mathrm{E}-01$ \\
\hline 4 & & $1.40 \mathrm{E}+04$ & 52 & 0.0020 & 0.56 & 0.20 & 0.00 & $1.85 E+00$ \\
\hline 5 & & $1.90 E+03$ & 5.7 & 0.0030 & 0.54 & 0.30 & 0.00 & 3.91E-01 \\
\hline 6 & & $4.80 \mathrm{E}+03$ & 12 & 0.0025 & 0.48 & 0.22 & 0.00 & $8.14 E-01$ \\
\hline 7 & & $3.10 E+03$ & 9 & 0.0029 & $\overline{0.73}$ & 0.36 & 0.00 & 5.66E-01 \\
\hline 8 & & $7.90 E+03$ & 15 & 0.0019 & 0.50 & 0.19 & 0.00 & $1.11 \mathrm{E}+00$ \\
\hline 9 & & $2.60 E+03$ & 7.3 & 0.0028 & 0.59 & 0.24 & 0.00 & 3.91E-01 \\
\hline 10 & & $2.00 \mathrm{E}+03$ & $\overline{6.3}$ & 0.0032 & 2.29 & 1.08 & 0.00 & 3.49E-01 \\
\hline 11 & & $5.60 \mathrm{E}+03$ & 16 & 0.0029 & 0.57 & 0.27 & 0.00 & $9.81 \mathrm{E}-01$ \\
\hline 12 & & $1.30 E+02$ & nd & nd & 0.88 & 0.34 & 0.00 & $1.86 \mathrm{E}-02$ \\
\hline 14 & & $1.80 \mathrm{E}+03$ & $\overline{6.3}$ & 0.0035 & 0.50 & 0.23 & 0.00 & $3.06 \mathrm{E}-01$ \\
\hline 15 & & $4.90 E+03$ & 10 & 0.0020 & 1.31 & 0.41 & 0.00 & 5.67E-01 \\
\hline$\overline{16}$ & & $4.40 E+03$ & 13 & 0.0030 & 0.55 & 0.27 & 0.00 & $7.99 \mathrm{E}-01$ \\
\hline 17 & & 1.60E+05 & 320 & 0.0020 & 1.93 & 0.79 & 0.00 & $2.42 E+01$ \\
\hline 18 & & $7.20 \mathrm{E}+03$ & 27 & 0.0038 & 1.03 & 0.48 & 0.00 & $1.24 \mathrm{E}+00$ \\
\hline 19 & & $1.20 \mathrm{E} \div 05$ & 240 & 0.0020 & 0.42 & 0.21 & 0.00 & $2.22 E+01$ \\
\hline 20 & & $1.30 \mathrm{E}+05$ & 24 & nd & $\overline{0.96}$ & $\overline{0.44}$ & 0.00 & $2.20 \mathrm{E}+01$ \\
\hline 21 & & $1.20 \mathrm{E}+03$ & 4.3 & 0.0036 & 0.56 & 0.34 & 0.00 & $2.70 \mathrm{E}-01$ \\
\hline 22 & & $8.60 E+02$ & 2.2 & 0.0026 & 2.24 & 1.07 & 0.00 & $1.52 E-01$ \\
\hline 23 & & $6.70 \mathrm{E}+02$ & nd & nd & 1.01 & 0.49 & 0.00 & $1.20 \mathrm{E}-01$ \\
\hline 24 & & $1.20 \mathrm{E}+03$ & nd & nd & 1.16 & 0.58 & 0.00 & 2.22E-01 \\
\hline 25 & & $4.20 \mathrm{E} \div 02$ & nd & nd & 1.05 & $\overline{0.44}$ & 0.00 & $6.51 \mathrm{E}-02$ \\
\hline 26 & & $2.90 \mathrm{E}+02$ & nd & nd & 0.85 & 0.39 & 0.00 & 4.92E-02 \\
\hline 27 & & $1.40 \mathrm{E}+04$ & 38 & 0.0027 & 0.37 & 0.16 & 0.00 & $2.24 \mathrm{E}+00$ \\
\hline 28 & & $4.20 \mathrm{E}+03$ & 19 & 0.0045 & 1.08 & 0.35 & 0.00 & 5.04E-01 \\
\hline 29 & & $2.80 \mathrm{E}+03$ & 20 & 0.0071 & 0.32 & 0.13 & 0.00 & 4.21E-01 \\
\hline 30 & & $3.20 \mathrm{E}+03$ & 9.6 & 0.0030 & 0.11 & 0.09 & 0.00 & $9.69 \mathrm{E}-01$ \\
\hline$\overline{31}$ & & $1.80 \mathrm{E}+03$ & 5.7 & 0.0032 & 5.94 & 2.14 & 0.33 & $3.70 \mathrm{E}-02$ \\
\hline$\overline{32}$ & & $9.00 \mathrm{E}+02$ & $\overline{4.3}$ & 0.0048 & 0.59 & 0.21 & 0.00 & 1.19E-01 \\
\hline 33 & & $1.20 \mathrm{E}+03$ & 2.9 & 0.0024 & 1.33 & 0.63 & 0.00 & $2.10 \mathrm{E}-01$ \\
\hline 34 & & $8.40 \mathrm{E}+02$ & 4.2 & 0.0050 & 0.50 & 0.21 & 0.00 & 1.31E-01 \\
\hline 35 & & $2.20 \mathrm{E}+03$ & 7.5 & 0.0034 & 3.69 & 1.46 & 0.00 & 3.22E-01 \\
\hline 37 & & $5.50 E+02$ & nd & nd & 1.69 & 0.74 & 0.00 & 8.91E-02 \\
\hline 38 & & $2.70 E+03$ & $\overline{9.8}$ & 0.0036 & $\overline{0.60}$ & 0.32 & 0.00 & 5.33E-01 \\
\hline 39 & & $1.80 \mathrm{E}+05$ & 530 & 0.0029 & $\overline{0.14}$ & 0.12 & 0.00 & $5.71 \mathrm{E}+01$ \\
\hline 40 & & $4.40 \mathrm{E}+03$ & 16.7 & 0.0038 & 0.20 & 0.06 & 0.00 & $4.88 \mathrm{E}-0.1$ \\
\hline 41 & & $5.30 \mathrm{E}+02$ & nd & nd & 0.39 & 0.18 & 0.00 & 9.05E-02 \\
\hline$\overline{43}$ & & $1.40 \mathrm{E}+03$ & 3 & 0.0021 & 1.54 & 0.62 & 0.00 & 2.09E-01 \\
\hline$\overline{44}$ & & $3.00 \mathrm{E}+03$ & 24.3 & 0.0081 & 1.50 & 0.61 & 0.00 & 4.51E-01 \\
\hline 45 & & $2.90 \mathrm{E}+03$ & 8.1 & 0.0028 & 0.60 & 0.28 & 0.00 & 5.01E-01 \\
\hline 46 & & $2.20 \mathrm{E}+02$ & nd & nd & 1.46 & 0.63 & 0.00 & $3.51 \mathrm{E}-02$ \\
\hline & & & & & & & No. Samples $>D L$ & 43 \\
\hline & & & & & & & $\begin{array}{c}\text { Ave } \\
\text { Median }\end{array}$ & $\frac{3.4 E+00}{3.9 E-01}$ \\
\hline & & & & & & & Std. Dev. & $1.1 \mathrm{E}+01$ \\
\hline & & & & & & & $95 \% \mathrm{Cl}$ & $3.3 E+00$ \\
\hline & & & & & & & Upper 95\% & $6.7 \mathrm{E}+00$ \\
\hline
\end{tabular}


Table E-5. Data for Small Herbivores Compartment $\left(C_{4}\right)$, YPG. Data for all samples listed, and descriptive statistics are shown. Only averages of replicates used in statistical calulations. Negative values indicate samples less than the detection limit, and the detection limit was the absolute value of the reported negative number. NA indicates a value is inappropriate, and nd indicates that no data were reported.

Pocket Mice, Carcass

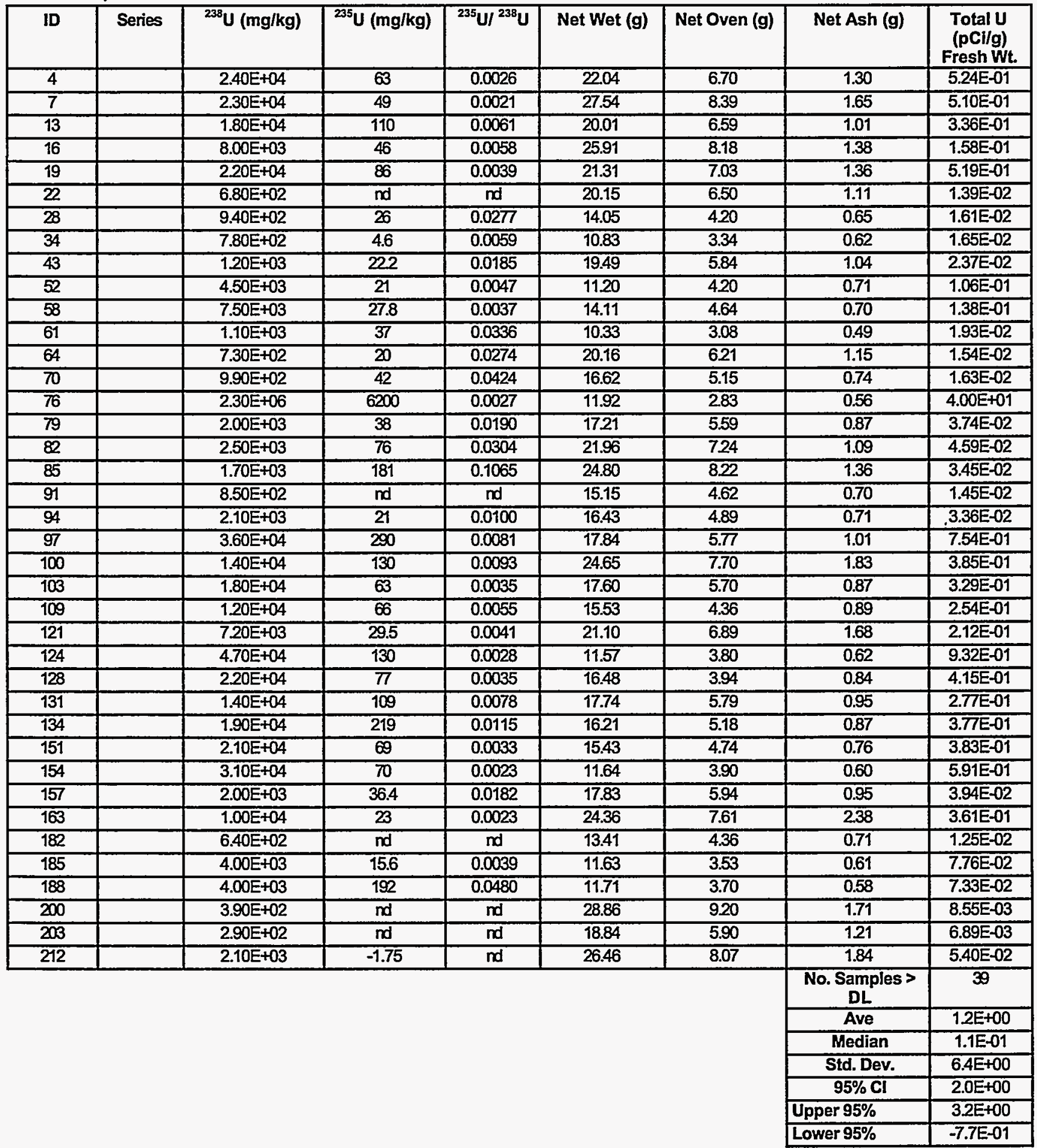


Table E-5, (cont)

Pocket Mice, Kidney

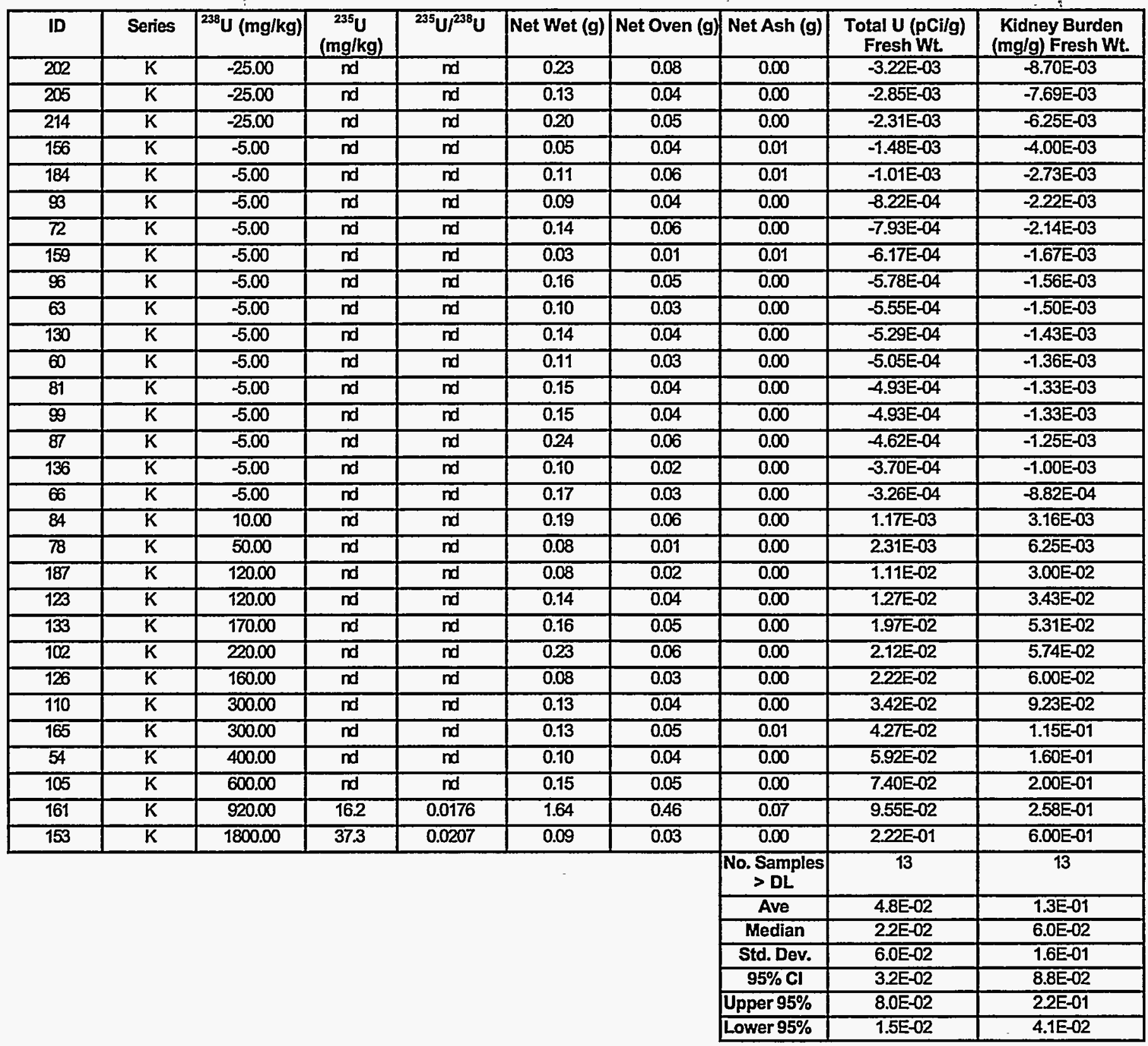


Table E-5, (cont)

Kangaroo Rat Carcass

\begin{tabular}{|c|c|c|c|c|c|c|c|c|}
\hline ID & Series & ${ }^{238} \mathrm{U}(\mathrm{mg} / \mathrm{kg})$ & ${ }^{235} \mathrm{U}(\mathrm{mg} / \mathrm{kg})$ & ${ }^{235} \mathrm{U} /{ }^{238} \mathrm{U}$ & Net Wet (g) & Net Oven (g) & Net Ash (g) & $\begin{array}{c}\text { Total U (pCi/g) } \\
\text { Fresh Wt. }\end{array}$ \\
\hline 1 & $\bar{A}$ & 2000.00 & 21 & 0.0105 & 34.74 & 13.65 & 2.44 & $5.20 \mathrm{E}-02$ \\
\hline$\overline{1}$ & $\bar{B}$ & 2500.00 & 20 & 0.0080 & 34.74 & 13.65 & 2.44 & $6.50 \mathrm{E}-02$ \\
\hline 1 & Ave. & 2250.00 & & & & & & $5.85 E-02$ \\
\hline 40 & $A$ & 2200.00 & 6.3 & 0.0029 & 35.21 & 9.40 & 2.63 & $6.08 \mathrm{E}-02$ \\
\hline 40 & $\bar{B}$ & 29000.00 & 52 & 0.0018 & 35.21 & 9.40 & 2.63 & 8.01E-01 \\
\hline 40 & Ave. & 15600.00 & & & & & & 4.31E-01 \\
\hline 49 & $A$ & 420.00 & nd & nd & 34.45 & 8.88 & 4.12 & $1.86 \mathrm{E}-02$ \\
\hline 49 & B & 600.00 & nd & nd & 34.45 & $\overline{8.88}$ & 4.12 & 2.65E-02 \\
\hline 49 & Ave. & 510.00 & & & & & & 2.26E-02 \\
\hline$\overline{67}$ & $A$ & 1.80E+03 & 3.6 & 0.0020 & 33.31 & 8.57 & 1.51 & $3.02 E-02$ \\
\hline 67 & $\bar{B}$ & $1.40 \mathrm{E}+03$ & 81 & 0.0579 & 33.31 & 8.57 & 0.96 & 1.49E-02 \\
\hline 67 & Ave. & $1.60 \mathrm{E}+03$ & NA & NA & NA & NA & NA & $2.26 \mathrm{E}-02$ \\
\hline$\overline{148}$ & $A$ & 10000.00 & 35 & 0.0035 & 26.91 & 6.71 & $\overline{1.86}$ & 2.56E-01 \\
\hline 148 & $\bar{B}$ & 23000.00 & 44 & 0.0019 & 26.91 & 6.71 & 1.86 & 5.88E-01 \\
\hline 148 & Ave. & 16500.00 & NA & NA & NA & NA & NA & 4.22E-01 \\
\hline 166 & & $5.40 \mathrm{E}+03$ & 30 & 0.0056 & 36.00 & 10.84 & 293 & $1.63 E-01$ \\
\hline 191 & $\bar{A}$ & $2.80 E+02$ & nd & nd & 34.54 & 9.47 & 6.52 & $1.96 \mathrm{E}-02$ \\
\hline 191 & $B$ & $3.80 \mathrm{E}+02$ & nd & nd & 34.54 & 9.47 & 6.52 & 2.65E-02 \\
\hline 191 & Ave. & $3.30 \mathrm{E}+02$ & NA & $\overline{N A}$ & NA & $\mathrm{NA}$ & NA & $2.30 E-02$ \\
\hline 194 & & $-5.00 E+00$ & nd & nd & 36.84 & 9.85 & 3.07 & $-1.54 \mathrm{E}-04$ \\
\hline 197 & A & $3.10 \mathrm{E}+02$ & nd & nd & 78.31 & 20.37 & 8.66 & 1.27E-02 \\
\hline & & & & & & & $\begin{array}{c}\text { No. Samples > } \\
\text { DL }\end{array}$ & 8 \\
\hline & & & & & & & Ave & 1.4E-01 \\
\hline & & & & & & & Median & 4.1E-02 \\
\hline & & & & & & & Std. Dev. & $1.8 \mathrm{E}-01$ \\
\hline & & & & & & & $95 \% \mathrm{Cl}$ & 1.3E-01 \\
\hline & & & & & & & Upper 95\% & 2.7E-01 \\
\hline & & & & & & & Lower 95\% & $1.9 E-02$ \\
\hline
\end{tabular}

Kangaroo Rat Liver

\begin{tabular}{|c|c|c|c|c|c|c|c|c|}
\hline ID & Series & ${ }^{238} \mathrm{U}(\mathrm{mg} / \mathrm{kg})$ & ${ }^{235} \mathrm{U}$ (mg/kg) & ${ }^{235} \mathrm{U} \mathrm{C}^{238} \mathrm{U}$ & Net Wet (g) & Net Oven (g) & Net Ash (g) & $\begin{array}{c}\text { Total U (pci/g) } \\
\text { Fresh Wt. }\end{array}$ \\
\hline 195 & $L$ & -12.50 & nd & nd & 0.41 & 0.13 & 0.00 & $-4.63 \mathrm{E}-03$ \\
\hline 50 & $\mathbf{L}$ & -5.00 & nd & nd & 0.67 & 022 & 0.04 & $-1.10 \mathrm{E}-04$ \\
\hline 149 & $\mathbf{L}$ & 0.03 & -5.00 & nd & 0.61 & 0.19 & 0.03 & $3.46 E-06$ \\
\hline 68 & $L$ & 1200 & nd & nd & 1.37 & 0.37 & 0.00 & 4.44E-03 \\
\hline 41 & $\bar{L}$ & 260.00 & nd & nd & 1.66 & 0.51 & 0.13 & $7.53 \mathrm{E}-03$ \\
\hline 2 & $\bar{L}$ & 930.00 & 21 & 0.0226 & 1.17 & $0 . \overline{37}$ & 0.04 & $1.18 \mathrm{E}-02$ \\
\hline 192 & $L$ & 43.00 & nd & nd & 0.75 & 021 & 0.00 & $1.59 \mathrm{E}-02$ \\
\hline 167 & $L$ & 120.00 & nd & nd & 0.87 & 0.42 & 0.06 & $4.44 E-02$ \\
\hline & & & & & & & No. Samples > DL & 6 \\
\hline & & & & & & & \begin{tabular}{|l} 
Ave \\
\end{tabular} & 1.4E-02 \\
\hline & & & & & & & Median & $9.6 \mathrm{E}-03$ \\
\hline & & & & & & & Std. Dev. & $1.6 \mathrm{E}-02$ \\
\hline & & & & & & & $95 \% \mathrm{Cl}$ & 1.3E-02 \\
\hline & & & & & & & Upper 95\% & 2.7E-02 \\
\hline & & & & & & & Lower 95\% & 1.3E-03 \\
\hline
\end{tabular}


Table E-5, (cont)

Kangaroo Rat Kidney

\begin{tabular}{|c|c|c|c|c|c|c|c|c|c|}
\hline ID & Series & ${ }^{238} \mathrm{U}(\mathrm{mg} / \mathrm{kg})$ & $\begin{array}{c}{ }^{235} \mathrm{U} \\
(\mathrm{mg} / \mathrm{kg})\end{array}$ & ${ }^{235} U /^{238} U$ & $\begin{array}{c}\text { Net Wet } \\
\text { (g) }\end{array}$ & $\begin{array}{l}\text { Net Oven } \\
\text { (g) }\end{array}$ & Net Ash (g) & $\begin{array}{c}\text { Total U (pCi/g) } \\
\text { Fresh Wt. }\end{array}$ & $\begin{array}{c}\text { Kidney Burden } \\
\text { (mg/g) Fresh } \\
\text { Wt. }\end{array}$ \\
\hline 3 & $\mathrm{~K}$ & 1500.00 & 36 & 0.0240 & 0.27 & 0.07 & 0.01 & 2.06E-02 & $5.56 E-02$ \\
\hline 42 & $\mathrm{~K}$ & 11000.00 & 198 & 0.0180 & 0.34 & 0.10 & 0.03 & 3.59E-01 & $9.71 E-01$ \\
\hline 51 & $\bar{K}$ & 4500.00 & nd & nd & 0.19 & 0.07 & 0.02 & $1.75 E-01$ & 4.74E-01 \\
\hline 6 & $\bar{K}$ & 120.00 & nd & 0.2700 & 0.27 & 0.07 & 0.00 & 1.15E-02 & 3.11E-02 \\
\hline 150 & $\bar{K}$ & 4400.00 & 259 & 0.0589 & 0.14 & 0.05 & 0.00 & 5.81E-01 & $1.57 E+00$ \\
\hline 168 & $\bar{K}$ & 3400.00 & 90 & 0.2500 & 0.25 & 0.09 & 0.01 & 4.53E-01 & $1.22 E+00$ \\
\hline 193 & $\mathrm{~K}$ & 160.00 & nd & 0.2500 & 0.25 & 0.07 & 0.00 & $1.66 \mathrm{E}-02$ & $4.48 E-02$ \\
\hline 196 & $\bar{K}$ & 57.00 & nd & 0.3400 & 0.34 & 0.08 & 0.00 & $4.96 E-03$ & $1.34 \mathrm{E}-02$ \\
\hline & & & & & & & No. Samples > DL & 8 & 8 \\
\hline & & & & & & & Ave & $2.0 \mathrm{E}-01$ & $5.5 E-01$ \\
\hline & & & & & & & Median & $9.8 \mathrm{E}-02$ & $2.6 \mathrm{E}-01$ \\
\hline & & & & & & & Std. Dev. & $2.3 E-01$ & $6.3 \mathrm{E}-01$ \\
\hline & & & & & & & $95 \% \mathrm{Cl}$ & $1.6 \mathrm{E}-01$ & $4.3 \mathrm{E}-01$ \\
\hline & & & & & & & Upper 95\% & $3.6 \mathrm{E}-01$ & $9.8 \mathrm{E}-01$ \\
\hline & & & & & & & Lower 95\% & $4.2 \mathrm{E}-02$ & $1.1 \mathrm{E}-01$ \\
\hline
\end{tabular}

Desert Iguana, Carcass

\begin{tabular}{|c|c|c|c|c|c|c|c|c|}
\hline ID & Series & ${ }^{238} \mathrm{U}(\mathrm{mg} / \mathrm{kg})$ & $\begin{array}{l}{ }^{235} \mathrm{U} \\
(\mathrm{mg} / \mathrm{kg})\end{array}$ & $\mathrm{U}-235 \Omega \mathrm{U}-238$ & $\begin{array}{c}\text { Net Wet } \\
\text { (g) }\end{array}$ & $\begin{array}{l}\text { Net Oven } \\
\text { (g) }\end{array}$ & Net Ash (g) & $\begin{array}{l}\text { Total U (pCilg) } \\
\text { Fresh Wt. }\end{array}$ \\
\hline 339 & & 4400.00 & 56 & 0.0127 & 11.22 & 4.00 & 2.40 & $3.5 E-01$ \\
\hline 353 & C & 11000.00 & 31 & 0.0028 & 61.14 & 15.61 & 4.64 & 3.1E-01 \\
\hline 359 & $\mathrm{CA}$ & 2600.00 & 7 & 0.0027 & 30.99 & 9.30 & 2.61 & 8.1E-02 \\
\hline 359 & $\overline{C B}$ & 2900.00 & 11 & 0.0038 & 30.99 & 9.30 & 2.61 & $9.0 E-02$ \\
\hline 359 & Ave. & 2750.00 & $\overline{N A}$ & NA & NA & NA & NA & $8.6 E-02$ \\
\hline 364 & & 2900.00 & 9 & 0.0031 & 3.55 & 0.74 & 0.44 & $1.3 E-01$ \\
\hline & & & & & & & No. Samples > DL & 4 \\
\hline & & & & & & & Ave & 2.2E-01 \\
\hline & & & & & & & Median & $22 E-01$ \\
\hline & & & & & & & Std. Dev. & $1.3 \mathrm{E}-01$ \\
\hline & & & & & & & $95 \% \mathrm{Cl}$ & $1.3 \mathrm{E}-01$ \\
\hline & & & & & & & Upper 95\% & 3.5E-01 \\
\hline & & & & & & & Lower 95\% & $9.3 E-02$ \\
\hline
\end{tabular}

Desert Iguana, Liver

\begin{tabular}{|c|c|c|c|c|c|c|c|c|}
\hline ID & Series & ${ }^{238} \mathrm{U}$ (mg/kg) & ${ }_{(\mathrm{mg} / \mathrm{kg})}^{235}$ & U-235/U-238 & $\begin{array}{l}\text { Net Wet } \\
\text { (g) }\end{array}$ & $\begin{array}{l}\text { Net Oven } \\
\text { (g) }\end{array}$ & Net Ash (g) & $\begin{array}{c}\text { Total U (pCi/g) } \\
\text { Fresh Wt. }\end{array}$ \\
\hline 354 & $\bar{L}$ & 6400.00 & 15 & 0.0023 & 1.37 & 0.35 & 0.00 & $6.0 \mathrm{E}-01$ \\
\hline 360 & $\bar{L}$ & 26.00 & nd & nd & 0.49 & 0.13 & 0.00 & $2.6 E-03$ \\
\hline & & & & & & & No. Samples > DL & 2 \\
\hline & & & & & & & Ave & $3.0 \mathrm{E}-01$ \\
\hline & & & & & & & Minimum & $2.6 \mathrm{E}-03$ \\
\hline & & & & & & & Maximum & $6.0 e-01$ \\
\hline
\end{tabular}


Table E-5. (cont)

White-throated Woodrat, Carcass

\begin{tabular}{|c|c|c|c|c|c|c|c|c|}
\hline ID & Series & ${ }^{238} \mathrm{U}(\mathrm{mg} / \mathrm{kg})$ & ${ }^{235} \mathrm{U}(\mathrm{mg} / \mathrm{kg})$ & $\begin{array}{l}\text { U-235I } \\
\text { U-238 }\end{array}$ & Net Wet (g) & Net Oven (g) & Net Ash (g) & $\begin{array}{c}\text { Total } \\
\text { U (pCi/g) } \\
\text { Fresh Wt. }\end{array}$ \\
\hline 10 & $\overline{C A}$ & 860000.00 & 1720 & 0.0020 & 153.55 & 40.83 & 12.72 & $2.64 E+01$ \\
\hline 25 & $\overline{\mathrm{CB}}$ & 530.00 & nd & nd & 133.96 & 35.51 & 10.81 & $1.58 \mathrm{E}-02$ \\
\hline 25 & $\overline{\mathrm{CA}}$ & 1100.00 & 1.2 & 0.0011 & 133.96 & 35.51 & 10.81 & $3.28 E-02$ \\
\hline 25 & Ave. & 815.00 & NA & NA & $\mathrm{NA}$ & $\mathrm{NA}$ & $\mathrm{NA}$ & 2.43E-02 \\
\hline$\overline{46}$ & $\bar{C}$ & 190.00 & nd & nd & 130.21 & 36.32 & 12.45 & $6.72 E-03$ \\
\hline 55 & $\bar{C}$ & 470.00 & nd & nd & 106.97 & 28.79 & 10.80 & 1.76E-02 \\
\hline 73 & $\bar{C}$ & 2700.00 & 6 & 0.0022 & 176.38 & 48.01 & 14.88 & $8.43 E-02$ \\
\hline 88 & C & 460.00 & nd & nd & 117.84 & 27.70 & 5.80 & 8.38E-03 \\
\hline 106 & $\overline{\mathrm{CB}}$ & 2700.00 & 28 & 0.0104 & 112.31 & $\overline{34.31}$ & 10.54 & $9.38 E-02$ \\
\hline 106 & $\overline{\mathrm{CA}}$ & 68000.00 & 240 & 0.0035 & 11231 & 34.31 & 10.54 & $2.36 \mathrm{E}+00$ \\
\hline 106 & Ave. & 35350.00 & $\overline{N A}$ & NA & NA & $\overline{N A}$ & $\mathrm{NA}$ & $1.23 \mathrm{E}+00$ \\
\hline 112 & $\mathrm{C}$ & 12000.00 & 27.6 & 0.0023 & 121.27 & 31.63 & 9.79 & $3.58 \mathrm{E}-01$ \\
\hline 115 & $\overline{\mathrm{CA}}$ & 73000.00 & 320 & 0.0044 & 96.94 & 26.23 & 7.47 & $2.08 \mathrm{E}+00$ \\
\hline 115 & $\overline{\mathrm{CB}}$ & 82000.00 & $\overline{\mathfrak{3}}$ & 0.0008 & 96.64 & 26.23 & 7.47 & $2.35 E+00$ \\
\hline$\overline{115}$ & Ave. & 77500.00 & NA & NA & $\mathrm{NA}$ & NA & $\overline{N A}$ & $2.21 E+\infty 0$ \\
\hline 118 & C & 21000.00 & 40 & 0.0019 & $\overline{150.06}$ & 53.66 & 12.09 & $6.26 \mathrm{E}-01$ \\
\hline 137 & $\bar{C}$ & 32000.00 & 58 & 0.0018 & $\overline{13261}$ & 35.61 & 12.26 & $1.09 \mathrm{E}+00$ \\
\hline 160 & $\overline{C A}$ & 18000.00 & 342 & 0.0019 & 116.30 & 44.46 & 17.34 & 9.93E-01 \\
\hline 160 & $\mathrm{CB}$ & 31000.00 & 220 & 0.0071 & 116.30 & 44.46 & 17.34 & 1.71E+00 \\
\hline 160 & Ave. & 24500.00 & $\overline{N A}$ & NA & NA & NA & $\overline{N A}$ & $1.35 \mathrm{E}+00$ \\
\hline 169 & $\mathrm{CB}$ & 26000.00 & 88 & 0.0034 & 165.30 & 42.91 & 15.01 & $8.74 E-01$ \\
\hline 169 & $\overline{\mathrm{CA}}$ & 30000.00 & 77 & 0.0026 & 165.30 & 42.91 & 15.01 & $1.01 \mathrm{E}+00$ \\
\hline 169 & Ave. & 28000.00 & $\overline{\mathrm{NA}}$ & NA & NA & NA & NA & $9.41 E-01$ \\
\hline 215 & $\mathrm{CA}$ & 3300.00 & 5.6 & 0.0017 & 108.38 & 30.30 & 14.77 & $1.66 \mathrm{E}-01$ \\
\hline 215 & CB & 4300.00 & 8.4 & 0.0020 & 108.38 & 30.30 & 14.77 & 2.17E-01 \\
\hline 215 & Ave. & 3800.00 & NA & NA & NA & NA & NA & 1.92E-01 \\
\hline & & & & & & & $\begin{array}{c}\text { No. Samples } \\
>\mathrm{DL}\end{array}$ & 14 \\
\hline & & & & & & & Ave & $2.5 E+00$ \\
\hline & & & & & & & Median & 4.9E-01 \\
\hline & & & & & & & Std. Dev. & $6.9 E+00$ \\
\hline & & & & & & & $95 \% \mathrm{CI}$ & $3.6 E+00$ \\
\hline & & & & & & & Upper 95\% & $6.1 E+00$ \\
\hline & & & & & & & Lower 95\% & $-1.2 E+00$ \\
\hline
\end{tabular}


Table E-5. (cont)

White-throated Woodrat, Liver

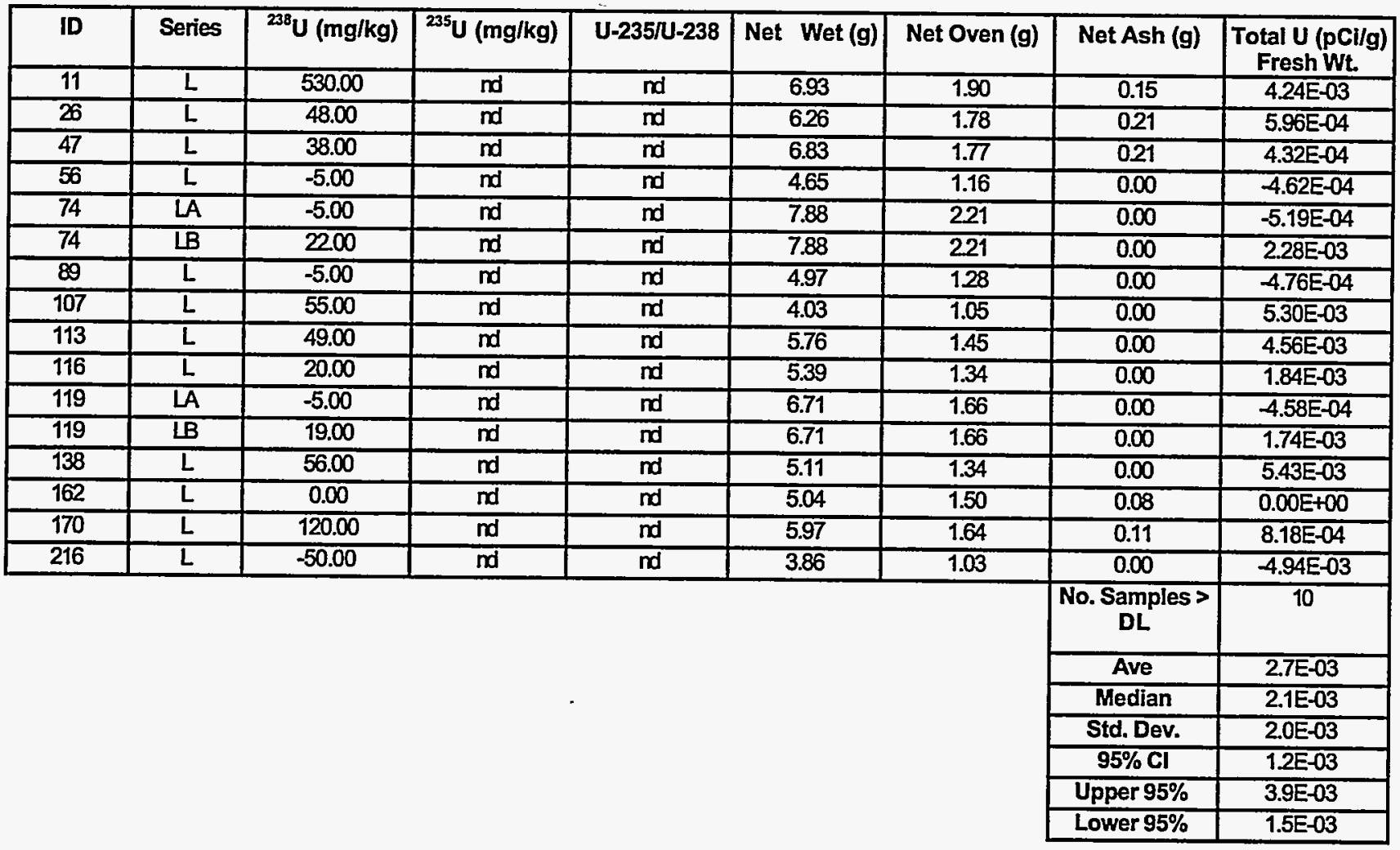

White-throated Woodrat, Kidneys

\begin{tabular}{|c|c|c|c|c|c|c|c|c|c|}
\hline ID & Series & ${ }^{238} \mathrm{U}(\mathrm{mg} / \mathrm{kg})$ & $\begin{array}{c}{ }^{235} \mathrm{U} \\
\text { (mg/kg) }\end{array}$ & $\begin{array}{l}\text { U-235I } \\
\text { U-238 }\end{array}$ & $\begin{array}{c}\text { Net } \\
\text { Wet }(g)\end{array}$ & $\begin{array}{l}\text { Net Oven } \\
\text { (g) }\end{array}$ & Net Ash (g) & $\begin{array}{c}\text { Total U } \\
\text { (pCi/g) Fresh } \\
\text { Wt. }\end{array}$ & $\begin{array}{l}\text { Kidney Burden } \\
\text { (mg/g-Kidney, } \\
\text { fresh wt.) }\end{array}$ \\
\hline 12 & $\bar{K}$ & 4400.00 & 18 & 0.0041 & 1.64 & 0.42 & 0.05 & $4.96 \mathrm{E}-02$ & $1.34 \mathrm{E}-01$ \\
\hline 27 & $\overline{\mathrm{K}}$ & 110.00 & nd & nd & 1.62 & 0.41 & 0.04 & $1.00 \mathrm{E}-03$ & $2.72 E-03$ \\
\hline 48 & $\bar{K}$ & 660.00 & nd & nd & 1.78 & 0.44 & 0.04 & 5.49E-03 & 1.48E-02 \\
\hline 57 & $\overline{\mathrm{K}}$ & -5.00 & nd & nd & 1.33 & $\overline{0.31}$ & 0.00 & $-4.31 E-04$ & $-1.17 E-03$ \\
\hline 75 & $\bar{K}$ & 14.00 & nd & nd & 1.92 & 0.47 & 0.00 & 1.27E-03 & $3.43 E-03$ \\
\hline 90 & $\bar{K}$ & -5.00 & nd & nd & 1.49 & 0.35 & 0.00 & $-4.35 \mathrm{E}-04$ & $-1.17 \mathrm{E}-03$ \\
\hline 108 & $\bar{K}$ & 720.00 & 12 & 0.0167 & 1.03 & 025 & 0.00 & 6.47E-02 & $1.75 \mathrm{E}-01$ \\
\hline 114 & $\bar{K}$ & 78.00 & nd & nd & 1.71 & 0.37 & 0.00 & $624 E-03$ & 1.69E-02 \\
\hline 117 & $\bar{K}$ & 75.00 & nd & nd & 1.20 & 0.28 & 0.00 & $6.48 E-03$ & 1.75E-02 \\
\hline 120 & $\bar{K}$ & 48.00 & nd & nd & 1.75 & 0.47 & 0.00 & 4.77E-03 & $1.29 E-02$ \\
\hline 139 & $\mathrm{~K}$ & 170.00 & nd & nd & 1.60 & 0.38 & 0.00 & 1.49E-02 & $4.04 E-02$ \\
\hline 161 & $\mathrm{~K}$ & 920.00 & 162 & 0.0176 & 1.64 & 0.46 & 0.07 & $1.45 E-02$ & $3.93 E-02$ \\
\hline 171 & $\bar{K}$ & 1100.00 & 30 & 0.0273 & 1.26 & $\overline{0.47}$ & 0.06 & 1.94E-02 & 524E-02 \\
\hline 217 & $\overline{\mathrm{K}}$ & 40.00 & nd & nd & 1.29 & 0.30 & 0.00 & 3.44E-03 & $9.30 \mathrm{E}-03$ \\
\hline & & & & & & & No. Samples > DL & 12 & 12 \\
\hline & & & & & & & Ave & $2.0 \mathrm{E}-02$ & $4.3 E-02$ \\
\hline & & & & & & & Median & $1.1 E-02$ & $1.7 E-02$ \\
\hline & & & & & & & Std. Dev. & $2.4 \mathrm{E}-02$ & $5.5 E-02$ \\
\hline & & & & & & & $95 \% \mathrm{Cl}$ & $1.4 E-02$ & $3.1 E-02$ \\
\hline & & & & & & & Upper 95\% & $3.4 \mathrm{E}-02$ & $7.4 \mathrm{E}-02$ \\
\hline & & & & & & & Lower 95\% & $6.1 E-03$ & $1.2 E-02$ \\
\hline
\end{tabular}


Table E-5. (cont.)

Desert Woodrat, Carcass

\begin{tabular}{|c|c|c|c|c|c|c|c|c|}
\hline ID & Series & ${ }^{238} \mathrm{U}(\mathrm{mg} / \mathrm{kg})$ & ${ }^{235} \mathrm{U}(\mathrm{mg} / \mathrm{kg})$ & $\begin{array}{l}\text { U-235I } \\
U-238\end{array}$ & Net Wet (g) & Net Oven (g) & Net Ash (g) & $\begin{array}{c}\text { Total U } \\
\text { (pCi/g) } \\
\text { Fresh Wt. }\end{array}$ \\
\hline 37 & $\mathrm{C}$ & 1500.00 & 3.5 & 0.0023 & 55.73 & 13.54 & 4.18 & 4.16E-02 \\
\hline 179 & $\mathrm{C}$ & 1300.00 & -0.09 & nd & 80.44 & 20.43 & 6.52 & $3.90 \mathrm{E}-02$ \\
\hline 197 & $\overline{\mathrm{CA}}$ & 310.00 & nd & nd & 78.31 & 20.37 & 8.66 & 1.27E-02 \\
\hline 197 & $\mathrm{CB}$ & 280.00 & nd & nd & 78.31 & 20.37 & 8.66 & 1.15E-02 \\
\hline 197 & Ave. & 295.00 & NA & NA & $\mathrm{NA}$ & NA & $\mathrm{NA}$ & 1.21E-02 \\
\hline 206 & C & 680.00 & nd & nd & 67.02 & 16.79 & 4.58 & 1.72E-02 \\
\hline 218 & $\mathrm{CA}$ & 350.00 & nd & nd & 72.06 & 19.67 & 13.16 & 2.37E-02 \\
\hline 218 & $\mathrm{CB}$ & 1100.00 & 142 & 0.0129 & 72.06 & 19.67 & 13.16 & 7.43E-02 \\
\hline 218 & Ave. & 725.00 & NA & $\mathrm{NA}$ & NA & $\mathrm{NA}$ & $\mathrm{NA}$ & $4.90 E-02$ \\
\hline & & & & & & & $\begin{array}{c}\text { No. Samples > } \\
\text { DL }\end{array}$ & 5 \\
\hline & & & & & & & Ave & 3.2E-02 \\
\hline & & & & & & & Median & $3.9 \mathrm{E}-02$ \\
\hline & & & & & & & Std. Dev. & 1.6E-02 \\
\hline & & & & & & & $95 \% \mathrm{Cl}$ & $1.4 \mathrm{E}-02$ \\
\hline & & & & & & & Upper $95 \%$ & $4.6 \mathrm{E}-02$ \\
\hline & & & & & & & Lower 95\% & $1.8 \mathrm{E}-02$ \\
\hline
\end{tabular}

Desert Woodrat, Liver

\begin{tabular}{|c|c|c|c|c|c|c|c|c|}
\hline ID & Series & ${ }^{238} \mathrm{U}(\mathrm{mg} / \mathrm{kg})$ & ${ }^{235} \mathrm{U}(\mathrm{mg} / \mathrm{kg})$ & $\begin{array}{l}\text { U-235I } \\
\text { U-238 }\end{array}$ & Net Wet (g) & Net Oven (g) & Net Ash (g) & $\begin{array}{c}\text { Total U } \\
\text { (pCi/g) } \\
\text { Fresh Wt. }\end{array}$ \\
\hline 38 & $L$ & 7200 & nd & nd & 1.73 & 0.47 & 0.09 & 1.39E-03 \\
\hline 180 & $\mathrm{~L}$ & 81.00 & nd & nd & 3.51 & 1.00 & 0.10 & $8.54 E-04$ \\
\hline 198 & $\mathrm{~L}$ & -50.00 & nd & nd & 4.56 & 1.18 & 0.00 & $-4.79 E-03$ \\
\hline 207 & $\bar{L}$ & 0.00 & nd & nd & 3.56 & 0.97 & 0.00 & $0.00 \mathrm{E}+00$ \\
\hline 219 & $\bar{L}$ & -2.50 & nd & $\overline{\text { nd }}$ & 4.03 & 1.04 & 0.00 & $-2.39 \mathrm{E}-04$ \\
\hline & & & & & & & $\begin{array}{c}\text { No. Samples > } \\
\text { DL }\end{array}$ & 2 \\
\hline & & & & & & & Ave & $1.1 \mathrm{E}-03$ \\
\hline & & & & & & & Std. Dev. & $3.8 E-04$ \\
\hline & & & & & & & Minimum & $8.5 E-04$ \\
\hline & & & & & & & Maximum & $1.4 \mathrm{E}-03$ \\
\hline
\end{tabular}

Desert Woodrat, Kidney

\begin{tabular}{|c|c|c|c|c|c|c|c|c|c|}
\hline ID & Series & ${ }^{238} \mathrm{U}(\mathrm{mg} / \mathrm{kg})$ & ${ }_{(\mathrm{mg} / \mathrm{kg})}^{235}$ & $\begin{array}{l}\text { U-235I } \\
\text { U-238 }\end{array}$ & $\begin{array}{l}\text { Net Wet } \\
\text { (g) }\end{array}$ & Net Oven (g) & Net Ash (g) & $\begin{array}{l}\text { Total U (pCi/g) } \\
\text { Fresh Wt. }\end{array}$ & $\begin{array}{c}\text { Kidney Burden } \\
\text { (mg/g-Kidney, } \\
\text { fresh wt.) }\end{array}$ \\
\hline 39 & $\mathrm{~K}$ & 78.00 & nd & nd & 0.92 & 022 & 0.08 & $2.51 \mathrm{E}-03$ & $6.78 \mathrm{E}-03$ \\
\hline 181 & $\bar{K}$ & -5.00 & nd & nd & 1.10 & 0.29 & 0.01 & $-1.68 E-05$ & $-4.55 E-05$ \\
\hline 199 & $K$ & 0.00 & nd & nd & 0.96 & 0.24 & 0.00 & $0.00 \mathrm{E}+00$ & $0.00 E+00$ \\
\hline 208 & $\bar{K}$ & 31.00 & nd & nd & 0.86 & 0.19 & 0.00 & 2.53E-03 & $6.85 E-03$ \\
\hline 220 & $K$ & -12.50 & nd & nd & 1.02 & 025 & 0.00 & $-1.13 E-03$ & $-3.06 E-03$ \\
\hline & & & & & & & $\begin{array}{c}\text { No. Samples > } \\
\text { DL }\end{array}$ & 2 & 2 \\
\hline & & & & & & & Ave & $2.5 E-03$ & $6.8 \mathrm{E}-03$ \\
\hline & & & & & & & Std. Dev. & $1.7 E-05$ & 4.7E-05 \\
\hline & & & & & & & Minimum & $2.5 E-03$ & $6.8 E-03$ \\
\hline & & & & & & & Maximum & $2.5 \mathrm{E}-03$ & $6.9 \mathrm{E}-03$ \\
\hline
\end{tabular}


Table E-6. Data for Large Herbivores Compartment $\left(C_{5}\right)$, YPG. Data for all samples listed, and descriptive statistics are shown. Negative values indicate samples less than the detection limit, and the detection limit was the absolute value of the reported negative number. NA indicates a value is inappropriate, and nd indicates that no data were reported.

Desert Cottontail Carcass, and Muscle

\begin{tabular}{|c|c|c|c|c|c|c|c|c|}
\hline ID & Series & ${ }^{238} \mathrm{U}(\mathrm{mg} / \mathrm{kg})$ & ${ }^{235} \mathrm{U}(\mathrm{mg} / \mathrm{kg})$ & ${ }^{235} \mathrm{U} /^{238} \mathrm{U}$ & Net Wet (g) & Net Oven (g) & Net Ash (g) & $\begin{array}{c}\text { Total U (pci/g) } \\
\text { Fresh Wt. }\end{array}$ \\
\hline 140 & C & 13.00 & nक & ก० & 48.53 & 13.44 & 3.09 & $3.06 \mathrm{E}-04$ \\
\hline 144 & C & 13.00 & no & no & 46.36 & 13.15 & 3.67 & 3.81E-04 \\
\hline 221 & $\overline{\mathrm{CA}}$ & 28.00 & तून & no & 395.45 & 220.85 & 26.17 & $6.86 E-04$ \\
\hline 297 & $\mathrm{CA}$ & 760.00 & 25 & 0.0033 & 274.62 & 60.11 & 14.30 & $1.46 \mathrm{E}-02$ \\
\hline$\overline{228}$ & $M$ & -2.50 & na & nd & 6.51 & 1.48 & 0.00 & $-2.10 E-04$ \\
\hline 143 & $\bar{M}$ & -5.00 & no & $\pi 0$ & $\overline{5.71}$ & 1.49 & 0.60 & $-1.94 E-04$ \\
\hline 300 & $\mathrm{MB}$ & -7.50 & กd & तथ & 6.93 & 1.66 & 0.47 & $-1.88 E-04$ \\
\hline 147 & $M$ & 60.00 & $\pi 0$ & $\pi 0$ & 7.49 & 1.93 & 0.11 & $3.26 \mathrm{E}-04$ \\
\hline 224 & $M$ & 300.00 & no & no & 7.90 & 1.82 & 0.00 & $2.56 E-02$ \\
\hline \multirow[t]{8}{*}{300} & MA & 2700000.00 & 13000 & 0.0048 & 6.93 & 1.66 & 0.47 & $6.78 \mathrm{E}+01$ \\
\hline & & & $\begin{array}{c}\text { With Sample } \\
300 \mathrm{MA}:\end{array}$ & $\begin{array}{c}\text { No. } \\
\text { Samples }> \\
\text { DL }\end{array}$ & 7 & $\begin{array}{c}\text { Without Sample } \\
\text { 300 MA: }\end{array}$ & $\begin{array}{c}\text { No. Samples > } \\
\text { DL }\end{array}$ & 6 \\
\hline & & & & Ave & $9.7 E+00$ & & Ave & $7.0 E-03$ \\
\hline & & & & Median & $6.9 E-04$ & & Median & 5.3E-04 \\
\hline & & & & Std. Dev. & $2.6 E+01$ & & Std. Dev. & $1.1 \mathrm{E}-02$ \\
\hline & & & & $95 \% \mathrm{Cl}$ & $1.9 E+01$ & & $95 \% \mathrm{Cl}$ & $8.6 E-03$ \\
\hline & & & & Upper 95\% & $2.9 E+01$ & & Upper $\mathbf{9 5 \%}$ & $1.6 \mathrm{E}-02$ \\
\hline & & & & Lower 95\% & $-9.3 E+00$ & & Lower 95\% & $-1.6 E-03$ \\
\hline
\end{tabular}

Desert Cottontail, Liver

\begin{tabular}{|c|c|c|c|c|c|c|c|c|}
\hline ID & Series & ${ }^{238} \mathrm{U}(\mathrm{mg} / \mathrm{kg})$ & ${ }^{235} \mathrm{U}(\mathrm{mg} / \mathrm{kg})$ & ${ }^{235} U I^{238} U$ & Net Wet (g) & Net Oven (g) & Net Ash (g) & $\begin{array}{c}\text { Total U (pCi/g) } \\
\text { Fresh Wt. }\end{array}$ \\
\hline 222 & $\overline{L B}$ & -7.50 & nd & nd & 16.48 & 4.14 & 0.00 & $-6.97 E-04$ \\
\hline 226 & $\overline{\mathrm{LB}}$ & -5.00 & nd & nd & 13.74 & 3.49 & 0.00 & $-4.70 \mathrm{E}-04$ \\
\hline 226 & $\overline{L A}$ & -2.50 & nd & nd & 13.74 & 3.49 & 0.00 & $-2.35 E-04$ \\
\hline 222 & $\overline{L A}$ & -2.50 & nd & nd & 16.48 & 4.14 & 0.00 & $-2.32 E-04$ \\
\hline 141 & $\bar{L}$ & -5.00 & nd & nd & 10.59 & 3.13 & 0.35 & $-6.11 E-05$ \\
\hline 145 & $\bar{L}$ & -5.00 & nd & nd & 10.87 & 329 & 0.27 & $4.60 \mathrm{E}-05$ \\
\hline 298 & $\bar{L}$ & 120.00 & nd & nd & 8.40 & 209 & 021 & $1.11 E-03$ \\
\hline & & & & & & & $\begin{array}{c}\text { No. Samples > } \\
\text { DL }\end{array}$ & 1 \\
\hline
\end{tabular}

Desert Cottontail, Kidney

\begin{tabular}{|c|c|c|c|c|c|c|c|c|c|}
\hline ID & Series & $\begin{array}{c}{ }^{238} \mathrm{U} \\
(\mathrm{mg} / \mathrm{kg})\end{array}$ & $\begin{array}{l}{ }^{235} \mathrm{U} \\
(\mathrm{mg} / \mathrm{kg})\end{array}$ & ${ }^{235} \mathrm{U} \mathrm{U}^{238} \mathrm{U}$ & $\begin{array}{c}\text { Net Wet } \\
\text { (g) }\end{array}$ & $\begin{array}{l}\text { Net Oven } \\
\text { (g) }\end{array}$ & Net Ash (g) & $\begin{array}{l}\text { Total U (pCi/g) } \\
\text { Fresh Wt. }\end{array}$ & $\begin{array}{l}\text { Kidiney Burden } \\
\text { (mg/g) Fresh } \\
\text { Wt. }\end{array}$ \\
\hline 227 & $\bar{K}$ & -2.50 & nd & nd & 5.99 & 120 & 0.00 & $-1.85 E-04$ & $-5.01 \mathrm{E}-04$ \\
\hline 142 & $\overline{\mathrm{K}}$ & -5.00 & nd & nd & 3.33 & 1.24 & 0.30 & $-1.67 E-04$ & $-4.50 E-04$ \\
\hline 146 & $\bar{K}$ & -5.00 & nd & nd & 3.94 & 1.14 & 0.18 & $-8.45 E-05$ & $-2.28 E-04$ \\
\hline 299 & $\bar{K}$ & 390.00 & nd & nd & 3.77 & 0.89 & 0.10 & $3.83 E-03$ & $1.03 E-02$ \\
\hline 223 & $\bar{K}$ & 160.00 & nd & nd & 8.21 & 2.26 & 0.00 & $1.63 \mathrm{E}-02$ & 4.40E-02 \\
\hline & & & & & & & No. Samples > DL & 2 & 2 \\
\hline & & & & & & & Ave & $1.0 \mathrm{E}-02$ & $2.7 E-02$ \\
\hline & & & & & & & Std. Dev. & $8.8 E-03$ & $2.4 E-02$ \\
\hline & & & & & & & Minimum & $3.80 \mathrm{E}-03$ & $1.00 E-02$ \\
\hline & & & & & & & Maximum & $1.60 E-02$ & $4.40 E-02$ \\
\hline
\end{tabular}


Table E-6, (cont)

Black-tailed Jack Rabbit, Carcass and Muscle

\begin{tabular}{|c|c|c|c|c|c|c|c|c|}
\hline ID & Series & ${ }^{238} \mathrm{U}$ (mg/kg) & ${ }^{235} \mathrm{U}$ (mg/kg) & ${ }^{235} U I^{238} U$ & Net Wet (g) & $\begin{array}{c}\text { Net Oven } \\
\text { (g) }\end{array}$ & Net Ash (g) & $\begin{array}{c}\text { Total U (pCi/g) } \\
\text { Fresh Wt. }\end{array}$ \\
\hline \multicolumn{9}{|c|}{ Carcass } \\
\hline 172 & C & -5.00 & nd & nd & 51.10 & 11.49 & 1.80 & $-6.52 E-05$ \\
\hline 301 & $\bar{C}$ & -2.50 & nd & nd & 55.31 & 13.28 & 3.72 & $-6.22 E-05$ \\
\hline 220 & $\bar{C}$ & 17.00 & nd & nd & 52.66 & 14.12 & 263 & 3.14E-04 \\
\hline 237 & $\mathrm{C}$ & 25.00 & nd & nd & 77.58 & 24.39 & 7.92 & 9.44E-04 \\
\hline 233 & $\overline{\mathrm{CA}}$ & 10.00 & 1.4 & 0.1400 & 95.40 & 23.19 & 7.17 & $2.78 E-04$ \\
\hline 233 & $\overline{\mathrm{CB}}$ & 16.00 & nd & nd & 95.40 & 23.19 & 7.17 & 4.45E-04 \\
\hline 233 & Ave. & 13.00 & $\overline{N A}$ & $\overline{N A}$ & NA & $\overline{N A}$ & $\overline{\mathrm{NA}}$ & $3.62 \mathrm{E}-04$ \\
\hline 229 & $\mathrm{CA}$ & 70.00 & nd & nd & 52.66 & 14.12 & 263 & $1.29 \mathrm{E}-03$ \\
\hline 229 & $\mathrm{CB}$ & 67.00 & nd & nd & 52.66 & 14.12 & 2.63 & $1.24 E-03$ \\
\hline 220 & Ave. & 68.50 & NA & NA & NA & NA & NA & 1.27E-03 \\
\hline \multicolumn{9}{|c|}{ Muscle } \\
\hline 175 & $M$ & -5.00 & nd & nd & 11.29 & 2.55 & 0.40 & $-6.55 \mathrm{E}-05$ \\
\hline 236 & $\bar{M}$ & -5.00 & nd & nd & 10.94 & 2.52 & 0.38 & $-6.43 E-05$ \\
\hline 240 & $\bar{M}$ & -2.50 & nd & nd & 23.16 & 5.23 & 0.90 & $-3.59 E-05$ \\
\hline 304 & $\bar{M}$ & 10.00 & nd & nd & 21.38 & 5.23 & 0.79 & 1.37E-04 \\
\hline \multirow[t]{8}{*}{232} & $\bar{M}$ & 11.00 & nd & nd & 11.73 & 2.78 & 0.40 & $1.39 E-04$ \\
\hline & & & & & & & No. Samples > DL & 6 \\
\hline & & & & & & & Ave & $5.3 E-04$ \\
\hline & & & & & & & Median & 3.4E-04 \\
\hline & & & & & & & Std. Dev. & 4.7E-04 \\
\hline & & & & & & & $95 \% \mathrm{Cl}$ & $3.7 E-04$ \\
\hline & & & & & & & Upper 95\% & $9.0 \mathrm{E}-04$ \\
\hline & & & & & & & Lower 95\% & 1.5E-04 \\
\hline
\end{tabular}

Black-tailed Jack Rabbit, Liver

\begin{tabular}{|c|c|c|c|c|c|c|c|c|}
\hline ID & Series & ${ }^{238} \mathrm{U}(\mathrm{mg} / \mathrm{kg})$ & ${ }^{235} \mathrm{U}(\mathrm{mg} / \mathrm{kg})$ & ${ }^{235} U /^{238} U$ & Net Wet (g) & Net Oven (g) & Net Ash (g) & $\begin{array}{c}\text { Total U (pCi/g) } \\
\text { Fresh Wt. }\end{array}$ \\
\hline 234 & L & -5.00 & nd & nd & 38.46 & 10.92 & 1.33 & $-6.40 \mathrm{E}-05$ \\
\hline 173 & $\mathrm{~L}$ & -5.00 & nd & nd & 32.81 & 10.89 & 0.88 & $-4.96 \mathrm{E}-05$ \\
\hline 238 & $\mathrm{~L}$ & -5.00 & nd & nd & 34.07 & 8.98 & 0.91 & $-4.94 E-05$ \\
\hline 302 & $\bar{L}$ & -2.50 & nd & $\overline{n d}$ & 59.73 & 15.62 & 1.35 & $-2.09 E-05$ \\
\hline 230 & $\bar{L}$ & 19.00 & nd & nd & 66.35 & 16.70 & 2.43 & 2.57E-04 \\
\hline & & & & & & & No. Samples $>$ DL & 1 \\
\hline
\end{tabular}

Black-tailed Jack Rabbit, Kidney

\begin{tabular}{|c|c|c|c|c|c|c|c|c|c|}
\hline ID & Series & ${ }^{238} \mathrm{U}(\mathrm{mg} / \mathrm{kg})$ & ${ }^{235} \mathrm{U}(\mathrm{mg} / \mathrm{kg})$ & ${ }^{235} \mathrm{U} / /^{238} \mathrm{U}$ & $\begin{array}{l}\text { Net Wet } \\
\text { (g) }\end{array}$ & $\begin{array}{l}\text { Net Oven } \\
\text { (g) }\end{array}$ & Net Ash (g) & $\begin{array}{c}\text { Total U (pCi/g) } \\
\text { Fresh Wt. }\end{array}$ & $\begin{array}{c}\text { Kidney } \\
\text { Burden } \\
\text { (mg/g) } \\
\text { Fresh W/t. }\end{array}$ \\
\hline 174 & $\mathrm{~K}$ & -5.00 & nd & nd & 1234 & 279 & 0.34 & $-5.10 \mathrm{E}-05$ & $-1.38 \mathrm{E}-04$ \\
\hline 235 & $\bar{K}$ & 20.00 & nd & nd & 16.94 & 6.95 & 0.33 & $1.44 \mathrm{E}-04$ & $3.90 \mathrm{E}-04$ \\
\hline 231 & $\mathrm{~K}$ & 11.00 & nd & nd & 19.46 & 4.12 & 0.70 & $1.46 \mathrm{E}-04$ & $3.96 \mathrm{E}-04$ \\
\hline 303 & $\bar{K}$ & 24.00 & nd & nd & 18.85 & 4.18 & 0.56 & 2.64E-04 & 7.13E-04 \\
\hline 239 & $\mathrm{~K}$ & 66.00 & nd & nd & 9.24 & 230 & 0.27 & $7.14 \mathrm{E}-04$ & 1.93E-03 \\
\hline & & & & & & & No. Samples > DL & 4 & 4 \\
\hline & & & & & & & Ave & $3.2 E-04$ & $8.6 \mathrm{E}-04$ \\
\hline & & & & & & & Median & 2.1E-04 & $5.5 \mathrm{E}-04$ \\
\hline & & & & & & & Std. Dev. & 2.7E-04 & $7.3 \mathrm{E}-04$ \\
\hline & & & & & & & $95 \% \mathrm{Cl}$ & $2.6 E-04$ & $7.2 E-04$ \\
\hline & & & & & & & Upper 95\% & $5.8 E-04$ & $1.6 \mathrm{E}-03$ \\
\hline & & & & & & & Lower 95\% & $5.2 E-05$ & 1.4E-04 \\
\hline
\end{tabular}


Table E-7. Data for Insectivores Compartment $\left(\mathbf{C}_{6}\right)$, YPG. Data for all samples listed, and descriptive statistics are shown. Only averages of replicates used in statistical calulations. Negative values indicate samples less than the detection limit, and the detection limit was the absolute value of the reported negative number. NA indicates a value is inappropriate, and nd indicates that no data were reported.

Lizards (Insectivores)

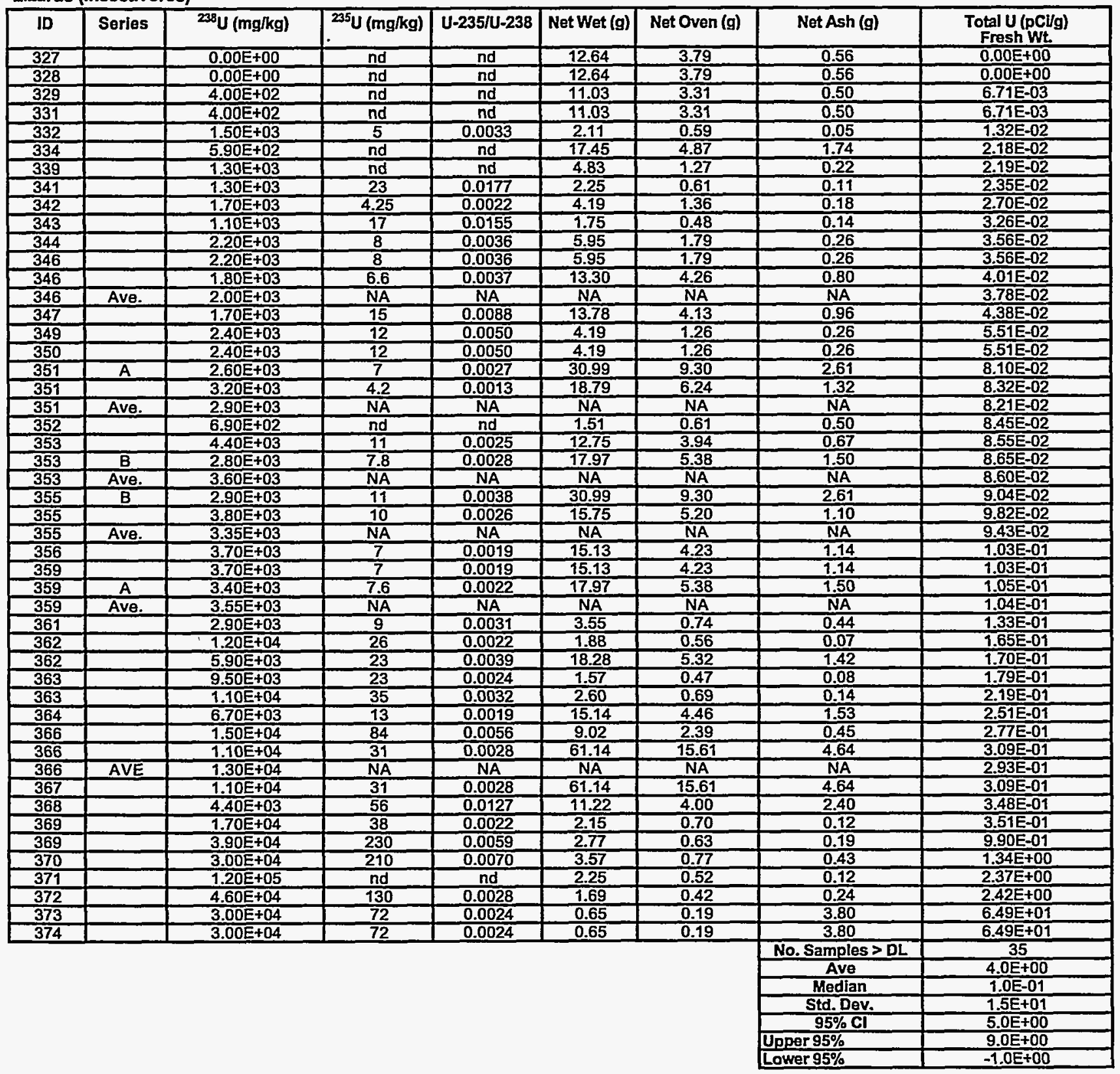


Table E-8. Data for comparison of washed and unwashed vegetation samples. Data for all samples listed, and descriptive statistics are shown. Negative values indicate samples less than the detection limit, and the detection limit was the absolute value of the reported negative number. NA indicates a value is inappropriate, and nd indicates that no data were reported.

Washed and Unwashed Veg. Comparison

\begin{tabular}{|c|c|c|c|c|c|c|c|c|}
\hline ID & ${ }^{238} \mathrm{U}(\mathrm{mg} / \mathrm{kg})$ & ${ }^{235} \mathrm{U}(\mathrm{mg} / \mathrm{kg})$ & Net Wet (g) & Net Oven (g) & Net Ash (g) & ${ }^{238} \mathrm{U}$ (pCi/g) & ${ }^{235} U$ (pCi/g) & ${ }^{235} \mathrm{U} /^{238} \mathrm{U}$ \\
\hline \multicolumn{9}{|c|}{ Unwashed } \\
\hline 250 & 96000 & 330 & 9.19 & 8.31 & 209 & $7.86 \mathrm{E}+00$ & 2.70E-02 & 0.0034 \\
\hline 256 & 1000000 & 2400 & 2.34 & 2.12 & 0.33 & $5.08 E+01$ & 1.22E-01 & 0.0024 \\
\hline$\overline{261}$ & 38000 & 200 & 1.9 & 1.72 & 0.32 & $2.30 E+00$ & 1.21E-02 & 0.0053 \\
\hline 275 & 62000 & 460 & 6.62 & 6.02 & 1.09 & $3.68 E+00$ & 2.73E-02 & 0.0074 \\
\hline 276 & 7700 & 14 & 10.48 & 9.54 & 2.33 & $6.16 \mathrm{E}-01$ & 1.12E-03 & 0.0018 \\
\hline 285 & 1100 & $\overline{\text { nd }}$ & 7.05 & $\overline{6.46}$ & 2.34 & $1.31 E-01$ & nd & nd \\
\hline 287 & 23000 & 56 & 9.35 & 8.56 & 1.84 & $1.63 E+00$ & $3.97 \mathrm{E}-03$ & 0.0024 \\
\hline 291 & 92000 & 380 & 9.78 & 9.03 & 3.66 & $1.24 \mathrm{E}+01$ & $5.12 E-02$ & 0.0041 \\
\hline 292 & 78000 & 190 & 5.67 & 5.27 & 1.87 & $9.26 E+00$ & $2.26 E-02$ & 0.0024 \\
\hline 293 & 62000 & 230 & 10.92 & 10.08 & 2.3 & $4.70 \mathrm{E}+00$ & $1.74 \mathrm{E}-02$ & 0.0037 \\
\hline \multirow{7}{*}{\multicolumn{5}{|c|}{. }} & $\begin{array}{c}\text { No. Samples } \\
\text { > DL }\end{array}$ & 10 & & \\
\hline & & & & & Ave & $9.3 E+00$ & & \\
\hline & & & & & Median & $4.2 E+00$ & & \\
\hline & & & & & Std. Dev. & $1.5 \mathrm{E}+01$ & & \\
\hline & & & & & $95 \% \mathrm{Cl}$ & $9.4 \mathrm{E}+00$ & & \\
\hline & & & & & Upper $95 \%$ & $1.9 \mathrm{E}+01$ & & \\
\hline & & & & & Lower $95 \%$ & $-2.3 E-02$ & & \\
\hline ID & ${ }^{238} \mathrm{U}(\mathrm{mg} / \mathrm{kg})$ & ${ }^{235} \mathrm{U}(\mathrm{mg} / \mathrm{kg})$ & Net Wet (g) & Net Oven (g) & Net Ash (g) & ${ }^{238} U$ (pCi/g) & ${ }^{235} \mathrm{U}(\mathrm{pCi} / \mathrm{g})$ & ${ }^{235} \mathrm{U} \mathrm{I}^{238} \mathrm{U}$ \\
\hline \multicolumn{9}{|c|}{ Washed } \\
\hline 250 & $\overline{\mathrm{BDL}}$ & $\overline{B D L}$ & 3.12 & & 0.72 & $\overline{\mathrm{BDL}}$ & $\overline{B D L}$ & nd \\
\hline 256 & $\mathrm{BDL}$ & BDL & 4.67 & & 0.66 & $\overline{B D L}$ & $\overline{B D L}$ & nd \\
\hline 261 & BDL & $\mathrm{BDL}$ & 7.6 & & 128 & $B D L$ & $\mathrm{BDL}$ & nd \\
\hline 275 & $\mathrm{BDL}$ & $\mathrm{BDL}$ & 3.67 & & 0.6 & BDL & $\mathrm{BDL}$ & nd \\
\hline 276 & $\overline{B D L}$ & $\mathrm{BDL}$ & 5.89 & & 1.31 & $\overline{B D L}$ & $\overline{B D L}$ & nd \\
\hline 285 & BDL & $\overline{B D L}$ & 6.29 & & 209 & $\overline{B D L}$ & $\overline{B D L}$ & nd \\
\hline 287 & $\mathrm{BDL}$ & $\mathrm{BDL}$ & 3.23 & & 0.64 & BDL & $\mathrm{BDL}$ & nd \\
\hline 291 & BDL & BDL & 5.69 & & 213 & $\overline{B D L}$ & $\overline{B D L}$ & nd \\
\hline 292 & $\overline{\mathrm{BDL}}$ & $\overline{\mathrm{BDL}}$ & 4.33 & & 1.43 & $\overline{\mathrm{BDL}}$ & $\overline{B D L}$ & nd \\
\hline 293 & $\overline{B D L}$ & $\overline{\text { BDL }}$ & 5.08 & & 10.7 & $\mathrm{BDL}$ & $\mathrm{BDL}$ & nd \\
\hline & & & & & $\begin{array}{c}\text { No. Samples } \\
>\mathrm{DL}\end{array}$ & 0 & & \\
\hline
\end{tabular}




\section{Appendix F}

Uncertainty Analyses for Dose Calculations

F-1 
Uncertainty in Dose Calculations

Dose rates in $\mathrm{mrad} / \mathrm{d}$

Forecast: Dose rate $(\mathrm{mrad} / \mathrm{d})$, Sunfish

Cell: D5

Summary:

Certainty Level is $95.00 \%$

Certainty Range is from $0.00 \mathrm{E}+0$ to $1.38 \mathrm{E}-3 \mathrm{mrad} / \mathrm{d}$

Display Range is from $-7.50 \mathrm{E}-4$ to $1.50 \mathrm{E}-3 \mathrm{mrad} / \mathrm{d}$

Entire Range is from $-7.04 \mathrm{E}-2$ to $2.94 \mathrm{E}-1 \mathrm{mrad} / \mathrm{d}$

After 10,000 Trials, the Std. Error of the Mean is 3.28E-5

Statistics:

Trials

Mean

Median (approx.)

Mode (approx.)

Standard Deviation

Variance

Skewness

Kurtosis

Coeff. of Variability

Range Minimum

Range Maximum

Range Width

Mean Std. Error
Value

10000

1.30E-04

5.96E-04

6.17E-04

3.28E-03

1.07E-05

71.54

$6,510.87$

25.18

$-7.04 \mathrm{E}-02$

2.94E-01

3.64E-01

3.28E-05

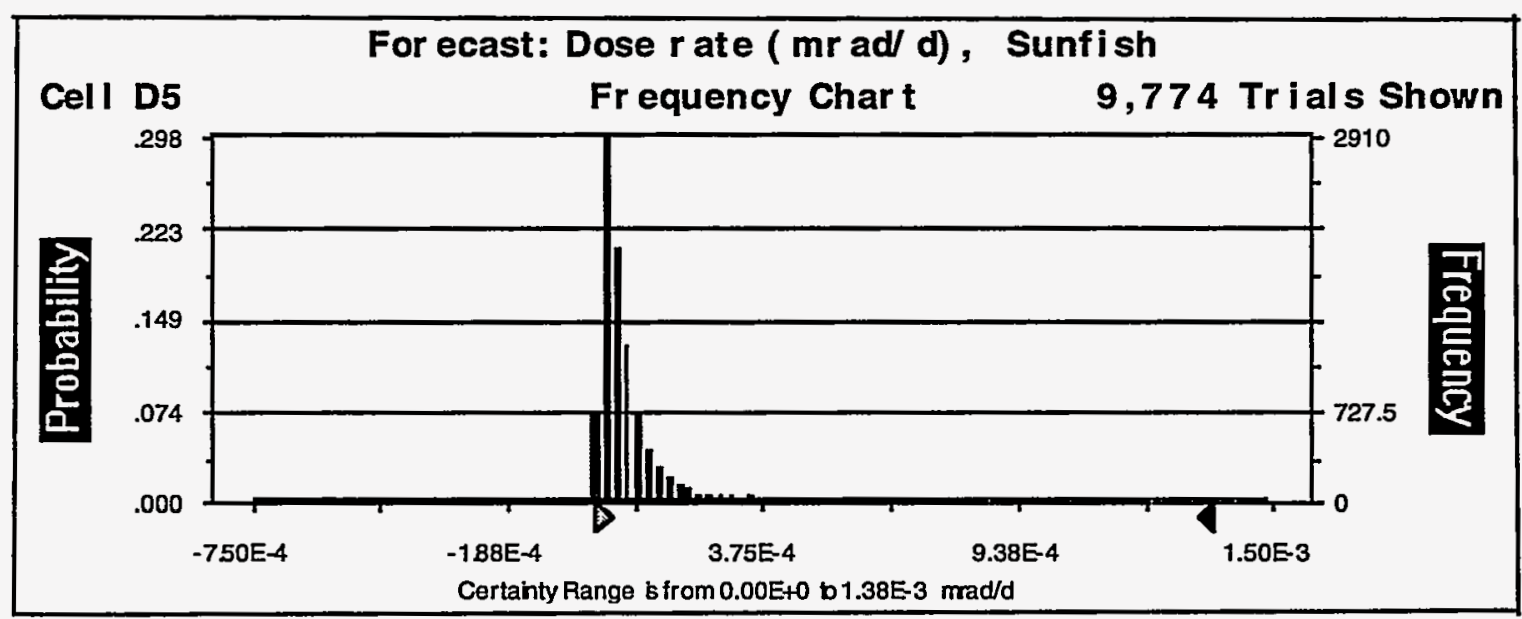


Summary:

Certainty Level is $95.00 \%$

Certainty Range is from $3.24 \mathrm{E}-6$ to $1.83 \mathrm{E}-4 \mathrm{mrad} / \mathrm{d}$

Display Range is from $0.00 \mathrm{E}+0$ to $5.50 \mathrm{E}-4 \mathrm{mrad} / \mathrm{d}$

Entire Range is from $-1.62 \mathrm{E}-1$ to $1.80 \mathrm{E}-2 \mathrm{mrad} / \mathrm{d}$

After 10,000 Trials, the Std. Error of the Mean is $1.66 \mathrm{E}-5$

Statistics:

Trials

Value

Mean

10000

Median (approx.)

$1.55 \mathrm{E}-05$

Mode (approx.)

2.70E-04

Standard Deviation

8.75E-04

Variance

1.66E-03

Skewness

2.77E-06

Kurtosis

Coeff. of Variability

$-92.25$

$9,013.48$

Range Minimum

107.04

Range Maximum

$-1.62 \mathrm{E}-01$

Range Width

$1.80 \mathrm{E}-02$

Mean Std. Error

1.80E-01

1.66E-05

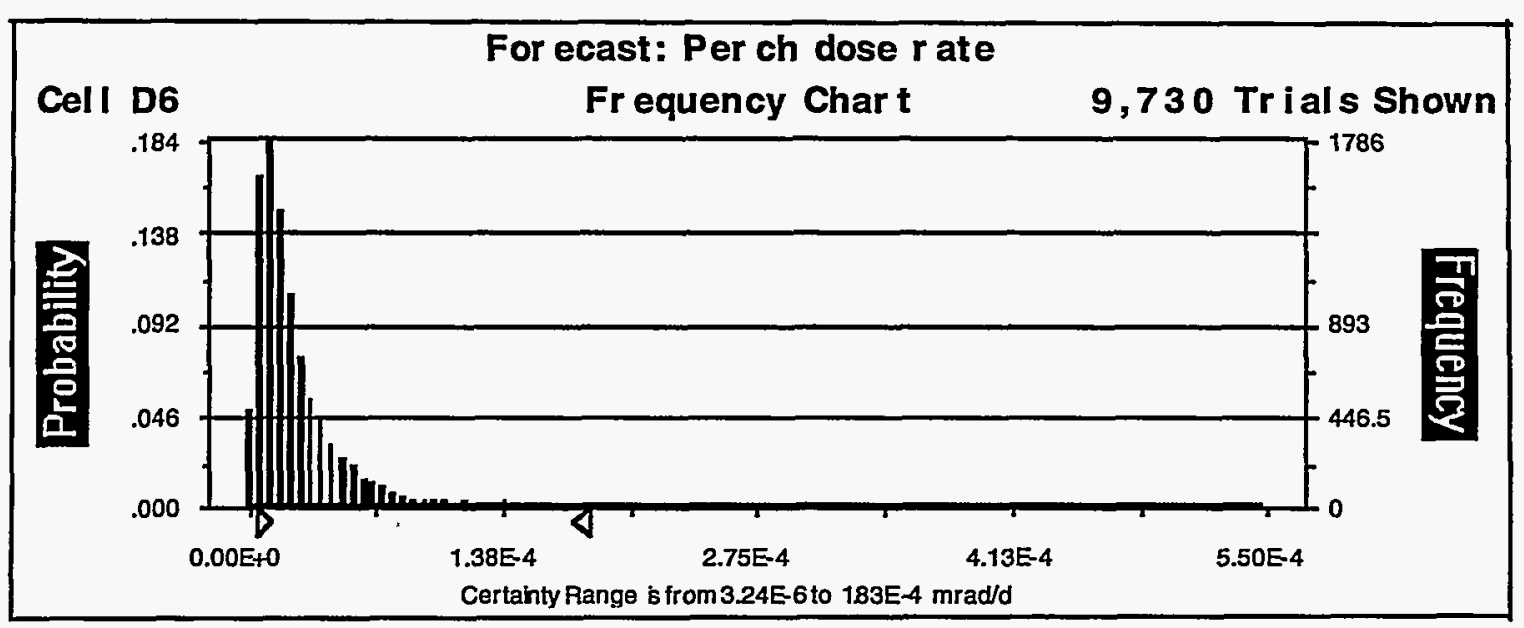




\section{Summary:}

Certainty Level is $95.00 \%$

Certainty Range is from $2.04 \mathrm{E}-7$ to $2.96 \mathrm{E}-6 \mathrm{mrad} / \mathrm{d}$

Display Range is from $-5.00 \mathrm{E}-6$ to $7.00 \mathrm{E}-6 \mathrm{mrad} / \mathrm{d}$

Entire Range is from $-1.19 \mathrm{E}-4$ to $1.30 \mathrm{E}-4 \mathrm{mrad} / \mathrm{d}$

After 10,000 Trials, the Std. Error of the Mean is 2.12E-8

Statistics:

Trials

Mean

Median (approx.)

Mode (approx.)

Standard Deviation

Variance

Skewness

Kurtosis

Coeff. of Variability

Range Minimum

Range Maximum

Range Width

Mean Std. Error
Value

10000

9.85E-07

8.39E-07

1.56E-06

2.12E-06

4.51E-12

6.24

$2,436.57$

2.16

$-1.19 \mathrm{E}-04$

1.30E-04

2.49E-04

2.12E-08

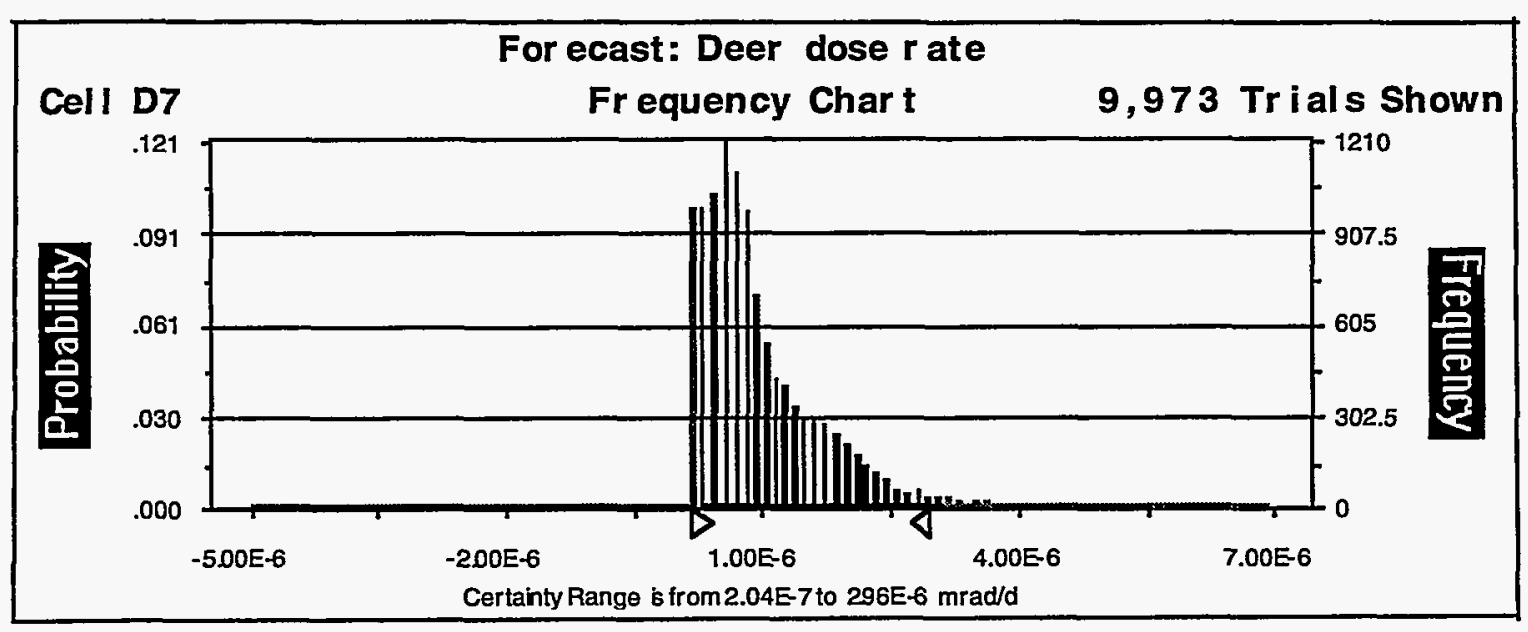


Summary:

Certainty Level is $93.32 \%$

Certainty Range is from $1.10 \mathrm{E}-4$ to $2.75 \mathrm{E}-3 \mathrm{mrad} / \mathrm{d}$

Display Range is from $0.00 \mathrm{E}+0$ to $2.75 \mathrm{E}-3 \mathrm{mrad} / \mathrm{d}$

Entire Range is from $9.18 \mathrm{E}-5$ to $5.02 \mathrm{E}-3 \mathrm{mrad} / \mathrm{d}$

After 10,000 Trials, the Std. Error of the Mean is 8.08E-6

Statistics:

Trials

Mean

Median (approx.)

Mode (approx.)

Standard Deviation

Variance

Skewness

Kurtosis

Coeff. of Variability

Value

10000

5.06E-04

2.53E-04

1.17E-04

8.08E-04

6.52E-07

3.20

12.31

1.60

Range Minimum

9.18E-05

Range Maximum

5.02E-03

Range Width

4.93E-03

Mean Std. Error

8.08E-06

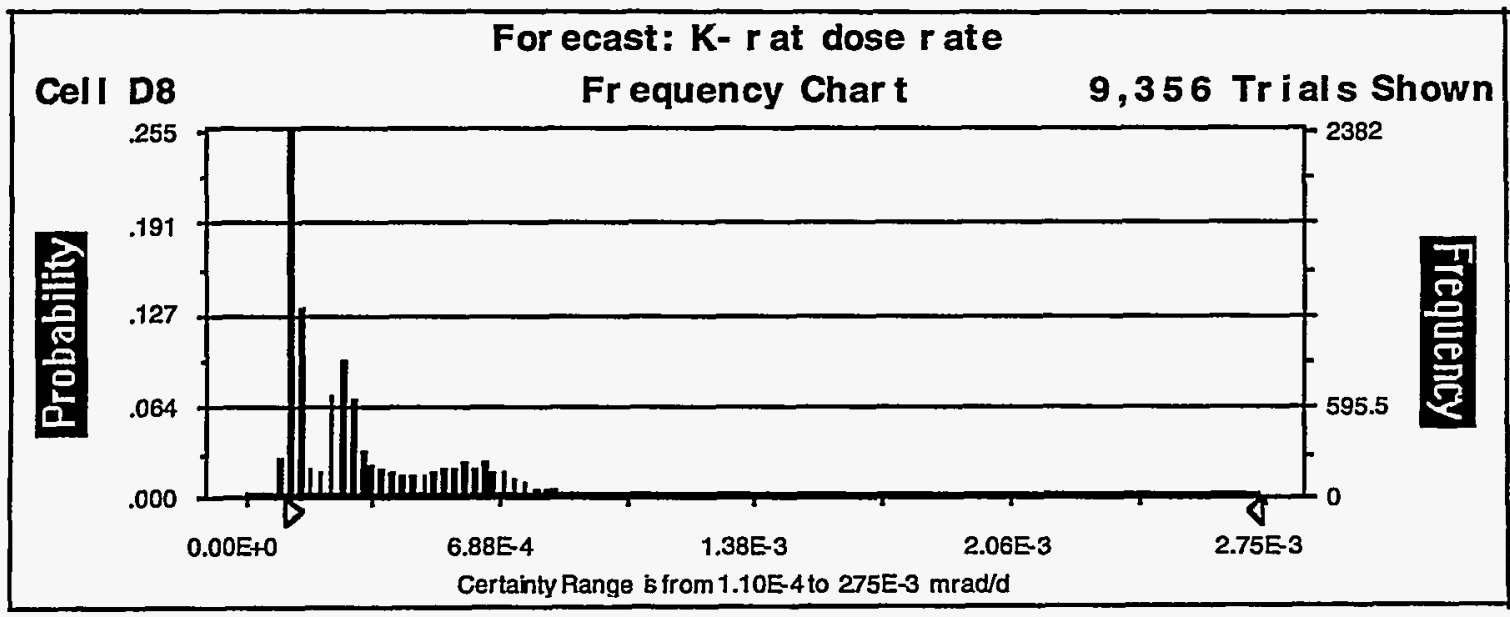


Summary:

Certainty Level is $95.00 \%$

Certainty Range is from $7.62 \mathrm{E}-4$ to + Infinity $\mathrm{mrad} / \mathrm{d}$

Display Range is from $0.00 \mathrm{E}+0$ to $4.50 \mathrm{E}-2 \mathrm{mrad} / \mathrm{d}$

Entire Range is from $6.73 \mathrm{E}-4$ to $6.12 \mathrm{E}-2 \mathrm{mrad} / \mathrm{d}$

After 10,000 Trials, the Std. Error of the Mean is 1.40E-4

Statistics:

Trials

Mean

Median (approx.)

Mode (approx.)

Standard Deviation

Variance

Skewness

Kurtosis

Coeff. of Variability

Range Minimum

Range Maximum

Range Width

Mean Std. Error
Value

10000

7.22E-03

1.63E-03

9.75E-04

1.40E-02

1.97E-04

2.63

8.41

1.94

6.73E-04

6.12E-02

6.05E-02

1.40E-04

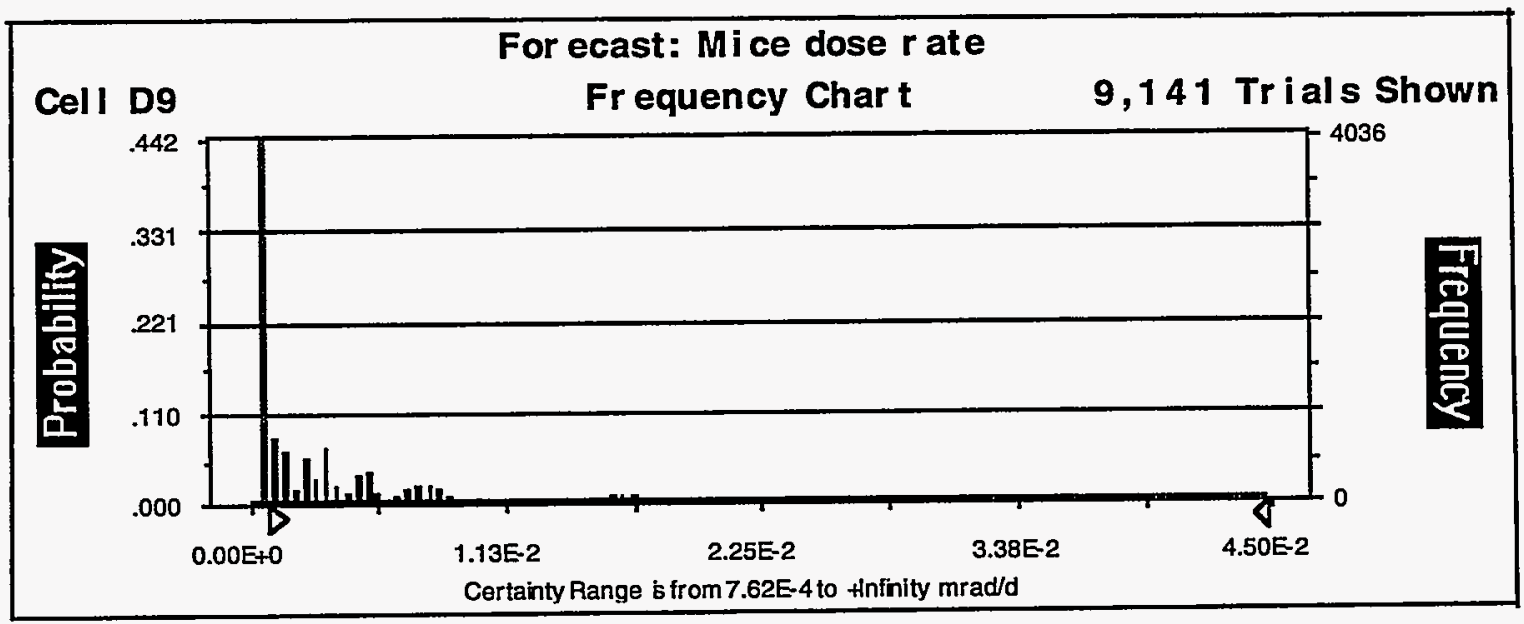


Summary:

Certainty Level is $94.36 \%$

Certainty Range is from $2.15 \mathrm{E}-4$ to $2.00 \mathrm{E}-2 \mathrm{mrad} / \mathrm{d}$

Display Range is from $0.00 \mathrm{E}+0$ to $2.00 \mathrm{E}-2 \mathrm{mrad} / \mathrm{d}$

Entire Range is from $-2.17 \mathrm{E}+1$ to $7.60 \mathrm{E}-1 \mathrm{mrad} / \mathrm{d}$

After 10,000 Trials, the Std. Error of the Mean is $3.04 \mathrm{E}-3$

Statistics:

Trials

Value

Mean

10000

Median (approx.)

$-1.14 \mathrm{E}-03$

Mode (approx.)

$-2.19 \mathrm{E}-02$

Standard Deviation

$-2.56 \mathrm{E}-02$

Variance

Skewness

3.04E-01

9.23E-02

$-70.45$

Kurtosis

Coeff. of Variability

$4,976.94$

$-266.61$

Range Minimum

$-2.17 E+01$

Range Maximum

7.60E-01

Range Width

2.25E+01

Mean Std. Error

3.04E-03

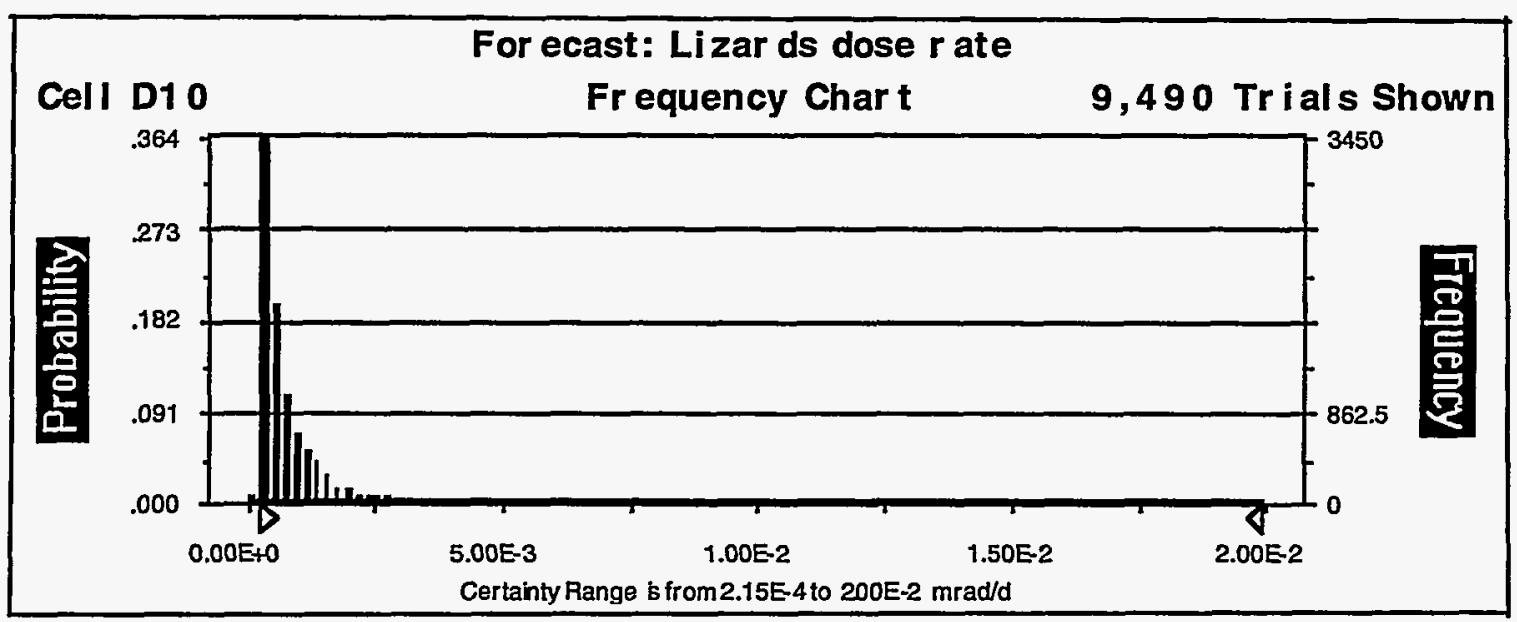


Forecast: Rabbits dose rate

Summary:

Certainty Level is $95.00 \%$

Certainty Range is from $1.34 \mathrm{E}-7$ to $3.68 \mathrm{E}-6 \mathrm{mrad} / \mathrm{d}$

Display Range is from $0.00 \mathrm{E}+0$ to $5.00 \mathrm{E}-6 \mathrm{mrad} / \mathrm{d}$

Entire Range is from $4.76 \mathrm{E}-8$ to $4.57 \mathrm{E}-6 \mathrm{mrad} / \mathrm{d}$

After 10,000 Trials, the Std. Error of the Mean is 9.63E-9

Statistics:

Trials

Value

Mean

10000

Median (approx.)

Mode (approx.)

1.57E-06

1.50E-06

Standard Deviation

3.42E-07

Variance

9.63E-07

Skewness

$9.28 \mathrm{E}-13$

Kurtosis

0.45

Coeff. of Variability

2.56

0.61

Range Minimum

4.76E-08

Range Maximum

4.57E-06

Range Width

4.53E-06

Mean Std. Error

9.63E-09

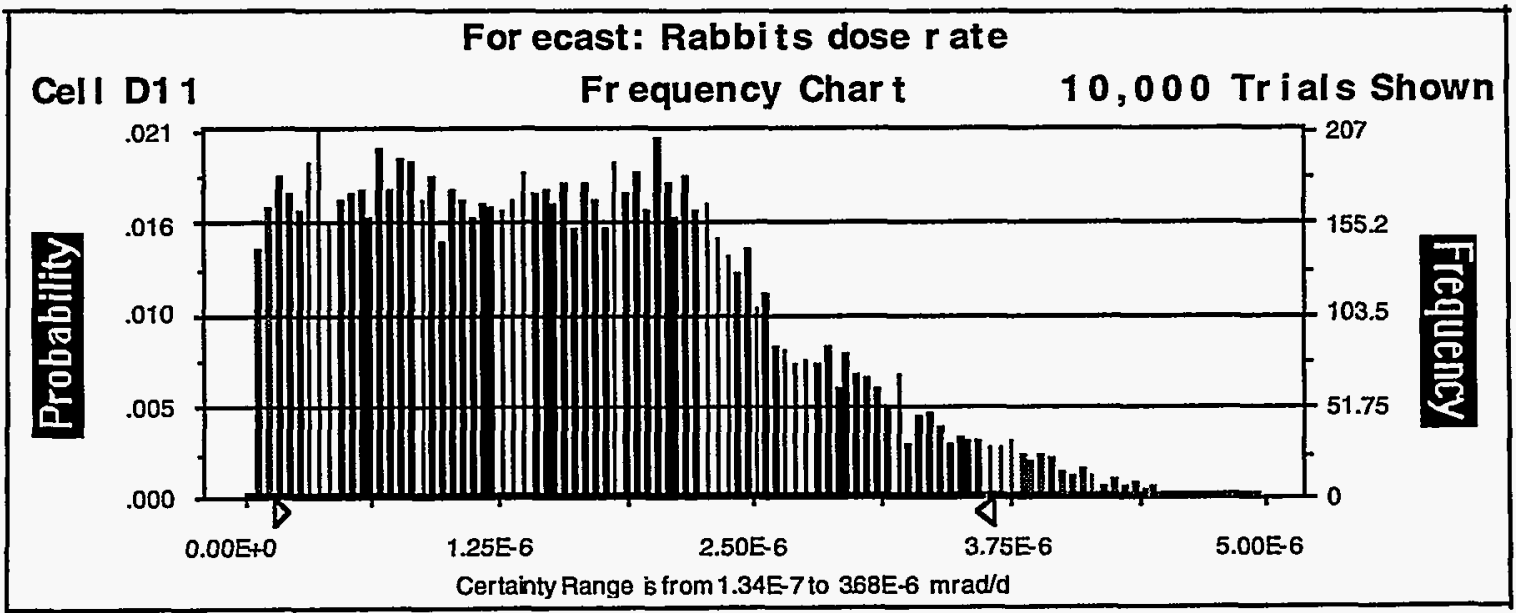


Summary:

Certainty Level is $93.18 \%$

Certainty Range is from $0.00 \mathrm{E}+0$ to $1.10 \mathrm{E}+0 \mathrm{mrad} / \mathrm{day}$

Display Range is from $-1.00 \mathrm{E}-1$ to $1.10 \mathrm{E}+0 \mathrm{mrad} / \mathrm{day}$

Entire Range is from $-6.25 \mathrm{E}+2$ to $1.64 \mathrm{E}+2 \mathrm{mrad} /$ day

After 10,000 Trials, the Std. Error of the Mean is 7.00E-2

Statistics:

Trials

Mean

Median (approx.)

Mode (approx.)

Standard Deviation

Variance

Skewness

Kurtosis

Coeff. of Variability

Range Minimum

Range Maximum

Range Width

Mean Std. Error
Value

10000

9.13E-02

$-2.36 \mathrm{E}-02$

$2.58 E+00$

$7.00 E+00$

$4.90 \mathrm{E}+01$

$-71.05$

$6,404.70$

76.66

$-6.25 E+02$

$1.64 \mathrm{E}+02$

$7.89 E+02$

7.00E-02

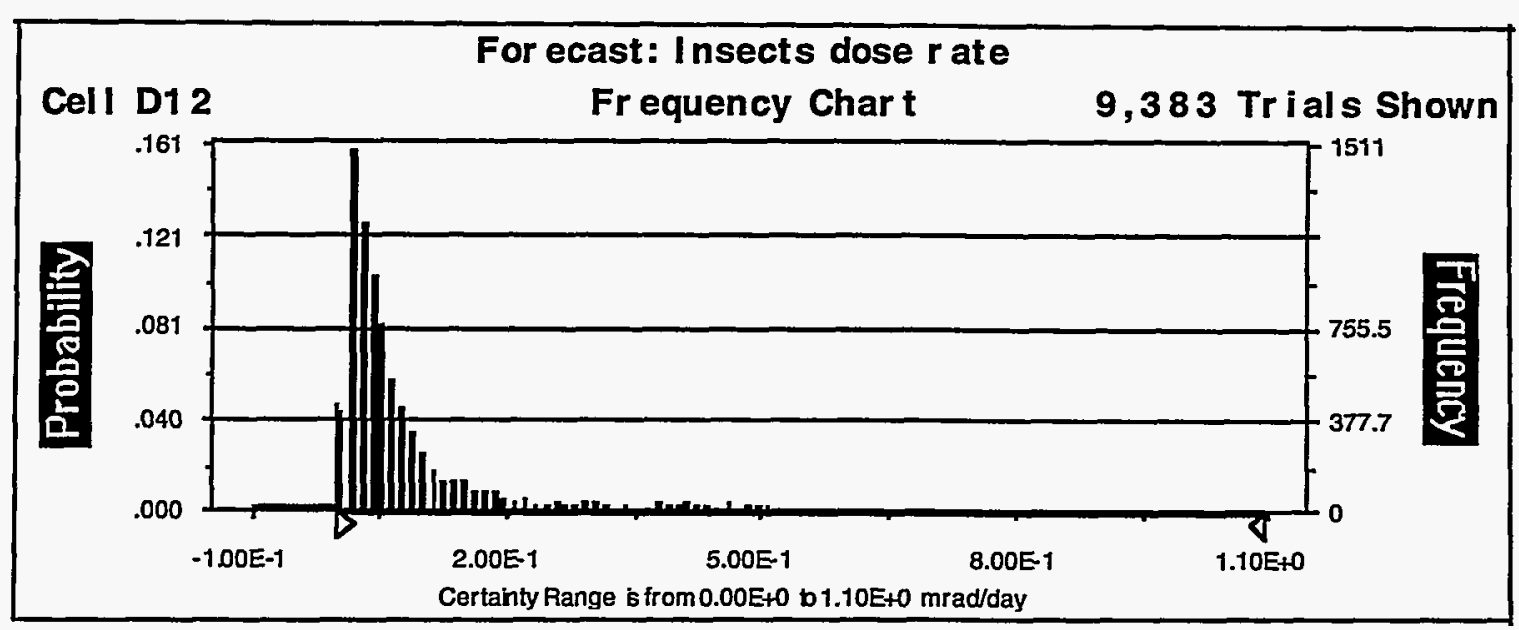


Summary:

Certainty Level is $94.97 \%$

Certainty Range is from $0.00 \mathrm{E}+0$ to $1.27 \mathrm{E}-2 \mathrm{mrad} / \mathrm{d}$

Display Range is from $0.00 E+0$ to $4.00 E-2 \mathrm{mrad} / \mathrm{d}$

Entire Range is from $-4.15 \mathrm{E}+0$ to $4.20 \mathrm{E}+0 \mathrm{mrad} / \mathrm{d}$

After 10,000 Trials, the Std. Error of the Mean is 5.98E-4

Statistics:

Trials

Mean

Median (approx.)

Mode (approx.)

Standard Deviation

Variance

Value

10000

3.84E-03

1.04E-02

$-1.75 \mathrm{E}-02$

5.98E-02

Skewness

3.57E-03

Kurtosis

Coeff. of Variability

1.02

$4,751.57$

Range Minimum

15.55

Range Maximum

$-4.15 E+00$

Range Width

$4.20 E+00$

Mean Std. Error

$8.34 \mathrm{E}+00$

5.98E-04

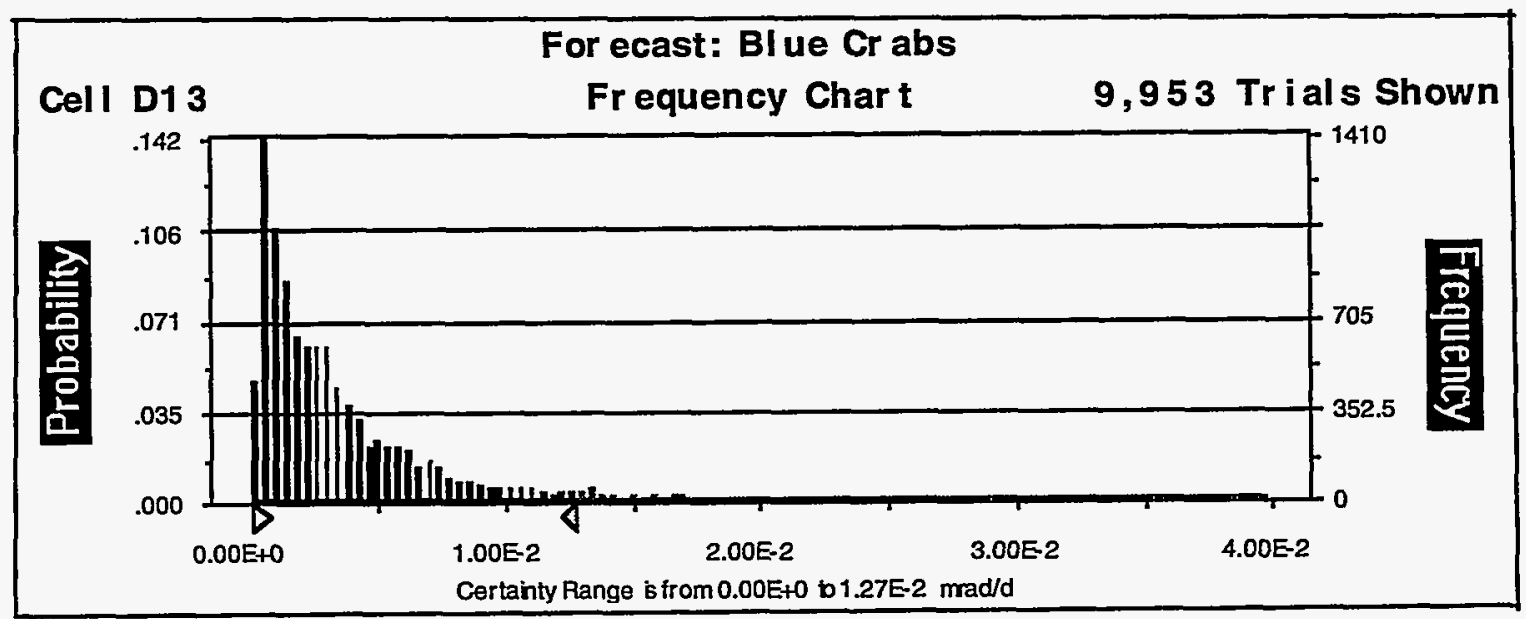

$F-10$ 


\section{Assumptions}

Assumption: Sunfish Concentration (pCi/g-wet)

Cell: B5

Custom distribution with parameters:

Single point

$1.00 \mathrm{E}-03$

Single point

Single point

Single point

Single point

Single point

Single point

Single point

Single point

Single point

Single point
$2.00 \mathrm{E}-03$

$3.00 \mathrm{E}-03$

4.00E-03

$5.00 \mathrm{E}-03$

$6.00 \mathrm{E}-03$

7.00E-03

$8.00 \mathrm{E}-03$

$9.00 \mathrm{E}-03$

$1.00 \mathrm{E}-02$

$5.00 \mathrm{E}-02$

Relative Prob.

0.170000

0.310000

0.098000

0.195000

0.098000

0.024400

0.024400

0.000010

0.024400

0.000010

0.040000

0.984220

Mean value in simulation was $4.98 \mathrm{E}-3$

Assumption: Perch Concentration (pCi/g-wet)

Cell: B6

Custom distribution with parameters:

Single point

Single point

Single point

Single point

Single point

Single point

Single point

Single point

Single point

Single point

Single point
1.00E-03

2.00E-03

3.00E-03

4.00E-03

5.00E-03

$6.00 \mathrm{E}-03$

7.00E-03

8.00E-03

$9.00 \mathrm{E}-03$

1.00E-02

5.00E-02

Total Relative Probability

Mean value in simulation was $3.52 \mathrm{E}-3$

Relative Prob.

0.161000

0.250000

0.265000

0.125000

0.045000

0.045000

0.035000

0.018000

0.000010

0.018000

0.008900

0.970910 
Custom distribution with parameters:

Single point

Single point

Single point

Single point

Single point

Single point

Single point

1.00E-04

2.00E-04

3.00E-04

4.00E-04

5.00E-04

6.00E-04

7.00E-04
Relative Prob.

0.211000

0.316000

0.215000

0.053000

0.000100

0.158000

0.053000

1.006100

Mean value in simulation was $3.01 \mathrm{E}-4$

Assumption: K-rats Concentration (pCi/g-wet)

Cell: B8

Custom distribution with parameters:

$\begin{array}{ll}\text { Single point } & 2.00 \mathrm{E}-02 \\ \text { Single point } & 4.00 \mathrm{E}-02 \\ \text { Single point } & 6.00 \mathrm{E}-02 \\ \text { Single point } & 8.00 \mathrm{E}-02 \\ \text { Single point } & 1.00 \mathrm{E}-01 \\ \text { Single point } & 5.00 \mathrm{E}-01\end{array}$

Relative Prob.

0.400000

0.267000

0.067000

0.067000

0.133000

0.067000

1.001000

Total Relative Probability

Mean value in simulation was $7.58 \mathrm{E}-2$

Assumption: Mice Concentration (pCi/g-wet)

Cell: B9

Custom distribution with parameters:

Single point

Single point

Single point

Single point

Single point

Single point

Single point

Single point

Single point

Single point

Single point

Single point
1.00E-01

2.00E-01

3.00E-01

4.00E-01

5.00E-01

6.00E-01

7.00E-01

8.00E-01

9.00E-01

$1.00 \mathrm{E}+00$

$2.00 \mathrm{E}+00$

$6.00 \mathrm{E}+00$
Relative Prob.

0.480000

0.065000

0.065000

0.097000

0.016000

0.081000

0.000100

0.016000

0.032000

0.032000

0.032000

0.096000

1.012100 
Mean value in simulation was $8.60 \mathrm{E}-1$

Assumption: Lizard Concentration (pCi/g-wet)

Cell: B10

Custom distribution with parameters:

$\begin{array}{ll}\text { Single point } & 1.00 \mathrm{E}-01 \\ \text { Single point } & 2.00 \mathrm{E}-01 \\ \text { Single point } & 3.00 \mathrm{E}-01 \\ \text { Single point } & 4.00 \mathrm{E}-01 \\ \text { Single point } & 1.00 \mathrm{E}+00 \\ \text { Single point } & 2.00 \mathrm{E}+00 \\ \text { Single point } & 6.00 \mathrm{E}+00\end{array}$

Relative Prob.

0.570000

0.163000

0.070000

0.090000

0.023000

0.023000

0.090000

1.029000

Total Relative Probability

Mean value in simulation was $7.32 \mathrm{E}-1$

Assumption: Rabbits Concentration (pCi/g-wet)

Cell: B11

Uniform distribution with parameters:
Minimum
3.10E-04
Maximum
1.50E-02

Mean value in simulation was $7.66 \mathrm{E}-3$

Assumption: Insects Concentration (pCi/g-wet)

Cell: B12

Custom distribution with parameters:

$\begin{array}{ll}\text { Single point } & 1.00 \mathrm{E}-01 \\ \text { Single point } & 2.00 \mathrm{E}-01 \\ \text { Single point } & 3.00 \mathrm{E}-01 \\ \text { Single point } & 4.00 \mathrm{E}-01 \\ \text { Single point } & 5.00 \mathrm{E}-01 \\ \text { Single point } & 6.00 \mathrm{E}-01 \\ \text { Single point } & 8.00 \mathrm{E}-01 \\ \text { Single point } & 9.00 \mathrm{E}-01 \\ \text { Single point } & 1.00 \mathrm{E}+00 \\ \text { Single point } & 2.00 \mathrm{E}+00 \\ \text { Single point } & 6.00 \mathrm{E}+00\end{array}$

Relative Prob.

0.163000

0.116000

0.090000

0.116000

0.070000

0.117000

0.023000

0.023000

0.070000

0.070000

0.140000

0.998000 
Mean value in simulation was $1.33 \mathrm{E}+0$

Assumption: Blue Crab Concentration (pCi/g-wet)

Cell: B13

Custom distribution with parameters:
Single point
$1.00 \mathrm{E}-03$
Single point
5.60E-02
Single point
$1.00 \mathrm{E}+00$
Single point
$2.00 \mathrm{E}+00$
Single point
$3.00 \mathrm{E}+00$
Single point
$4.00 \mathrm{E}+00$
Single point
$5.00 E+00$
Single point
$1.00 \mathrm{E}+01$
Single point
$2.00 E+01$

Relative Prob.

0.056000

0.000001

0.278000

0.222000

0.111100

0.056000

0.278000

0.170000

0.056000

Total Relative Probability

1.227101

Mean value in simulation was $4.52 \mathrm{E}+0$

\section{Assumption: Sunfish Body Mass (g)}

Cell: C5

Normal distribution with parameters:
Mean
$1.24 \mathrm{E}+01$
Standard Dev.
$6.80 \mathrm{E}+00$

Selected range is from -Infinity to +Infinity Mean value in simulation was $1.24 \mathrm{E}+1$

\section{Assumption: Perch Body Mass (g)}

Cell: $\mathbf{C 6}$

Normal distribution with parameters:
Mean
$3.06 E+01$
Standard Dev.
$1.48 \mathrm{E}+01$

Selected range is from -Infinity to +Infinity Mean value in simulation was $3.04 \mathrm{E}+1$ 
Normal distribution with parameters:

$\begin{array}{ll}\text { Mean } & 7.58 \mathrm{E}+01 \\ \text { Standard Dev. } & 2.40 \mathrm{E}+01\end{array}$

Selected range is from -Infinity to +Infinity Mean value in simulation was $7.58 \mathrm{E}+1$

Assumption: Kangaroo Body Mass (g)

Cell: $\mathbf{C 8}$

Normal distribution with parameters:

Mean

Standard Dev.

$3.27 \mathrm{E}+01$

4.10E+00

Selected range is from -Infinity to +Infinity

Mean value in simulation was $3.27 \mathrm{E}+1$

Cell: $C 9$

Normal distribution with parameters:

$$
\begin{array}{ll}
\text { Mean } & 2.56 \mathrm{E}+01 \\
\text { Standard Dev. } & 1.70 \mathrm{E}+00
\end{array}
$$

Selected range is from -Infinity to +Infinity Mean value in simulation was $2.56 \mathrm{E}+1$

\section{Assumption: Lizard Body Mass (g)}

Cell: $\mathrm{C10}$

Normal distribution with parameters:

$$
\begin{array}{ll}
\text { Mean } & 6.00 \mathrm{E}+01 \\
\text { Standard Dev. } & 2.00 \mathrm{E}+01
\end{array}
$$

Selected range is from -Infinity to +Infinity Mean value in simulation was $6.00 \mathrm{E}+1$ 
Assumption: Rabbit Body Mass (g)

Uniform distribution with parameters:
Minimum
$7.00 \mathrm{E}+02$
$1.50 \mathrm{E}+03$

Maximum

Mean value in simulation was $1.10 \mathrm{E}+3$

Assumption:Insect Body Mass (g)

Cell: C12

Normal distribution with parameters:
Mean
$2.00 \mathrm{E}+00$
Standard Dev.
$1.10 E+00$

Selected range is from -Infinity to +Infinity Mean value in simulation was $2.00 \mathrm{E}+0$

Assumption: Blue Crab Body Mass (g)

Cell: $\mathbf{C 1 3}$

Normal distribution with parameters:

Mean

$3.00 \mathrm{E}+02$

Standard Dev. $\quad 1.00 E+02$

Selected range is from -Infinity to +Infinity Mean value in simulation was $2.99 \mathrm{E}+2$

End of Assumptions 